Probing the regulation of cellular lipid metabolism by trans fatty acids and the lipid-sensitive ANGPTL4 protein

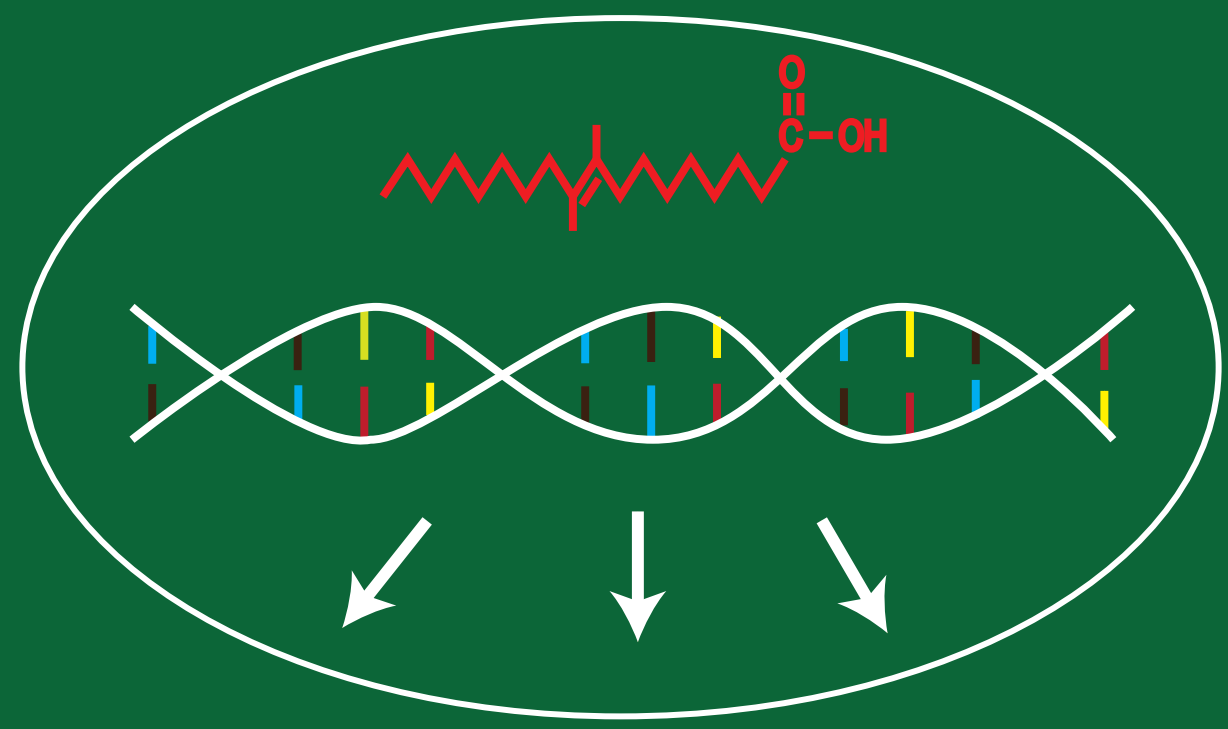

Antwi-Boasiako Oteng 



\section{Propositions}

1. Foam cell formation in mesenteric lymph nodes of Angptl4 ${ }^{-/-}$mice after high fat feeding is uncoupled from peritoneal ascites and acute inflammation.

(this thesis)

2. Safe targeting of ANGPTL4 against dyslipidaemia may lie in the partial but not full repression of this protein.

(this thesis)

3. Based on evidence at the cellular and molecular level, the harmful effects of trans fats are over-exaggerated.

4. Classification of fatty acids into saturated, cis- and trans-unsaturated fatty acids downplays the specificity and unique properties of every individual fatty acid.

5. Definition of a biological cell as "the basic unit of life" is an oversimplification of the complexity that a cell displays in the lab.

6. "Science communication to non-scientific audience" should be made a mandatory course at the Masters' and PhD level.

7. An expert is the person who has had ample time to right his wrongs.

8. In several parts of Africa today, a biomedical research scientist is a luxury.

Propositions belonging to the $\mathrm{PhD}$ thesis entitled:

"Probing the regulation of cellular lipid metabolism by trans fatty acids and the lipidsensitive ANGPTL4 protein"

Antwi-Boasiako Oteng

Wageningen, June 4, 2019 



\section{Probing the regulation of cellular lipid metabolism by trans fatty acids and the lipid-sensitive ANGPTL4 protein}

Antwi-Boasiako Oteng 


\section{Thesis Committee}

\section{Promotor}

Prof. Dr Sander Kersten

Professor of Molecular Nutrition

Chair of Nutrition, Metabolism and Genomics group

Division of Human Nutrition and Health

Wageningen University \& Research

\section{Other Members}

Prof. Dr Renger Witkamp, Wageningen University

Prof. Dr Matijn Katan, Vrije University Amsterdam

Prof. Dr Patrick Rensen, Leiden University

Prof. Dr Jogchum Plat, Maastricht University

This research was conducted under the auspices of the Graduate School VLAG (Advanced studies in Food Technology, Agrobiotechnology, Nutrition and Health Sciences). 


\title{
Probing the regulation of cellular lipid metabolism by trans fatty acids and the lipid-sensitive ANGPTL4 protein
}

\author{
Antwi-Boasiako Oteng
}

Thesis

submitted in fulfilment of the requirements for the degree of doctor at Wageningen University

by the authority of the Rector Magnificus

Prof. Dr A. P. J. Mol

in the presence of the

Thesis Committee appointed by the Academic Board

to be defended in public

on Tuesday June 4, 2019

at 11 a.m. in the Aula 


\section{Antwi-Boasiako Oteng}

Probing the regulation of cellular lipid metabolism by trans fatty acids and the lipid-sensitive ANGPTL4 protein

232 pages

PhD thesis, Wageningen University, Wageningen, The Netherlands (2019)

With references, with summary in English

ISBN: 978-94-6343-908-4

DOI: $10.18174 / 472448$ 


\section{CONTENTS}

\section{Chapter 1}

General Introduction

Chapter 2

Molecular effects of trans fatty acids

\section{Chapter 3}

Industrial trans fatty acids promote non-alcoholic fatty liver disease and stimulate SREBP2-mediated cholesterogenesis

\section{Chapter 4} 90

Feeding Angptl $4^{-/}$mice trans fat promotes foam cell formation in mesenteric lymph nodes without leading to ascites

Chapter 5

ANGPTL4 hypomorphic mice expressing truncated N-terminal ANGPTL4 exhibit lymphadenopathy and mild chylous ascites upon high fat feeding

\section{Chapter 6}

Muscle-specific inflammation induced by MCP-1 overexpression does not affect whole-body insulin sensitivity in mice

Chapter 7

General Discussion

Summary

Acknowledgements

About the Author 
Chapter 1 
General Introduction 


\section{Classification of fatty acids}

Fats are a class of lipids that consist of biomolecules such as phospholipids, cholesterol and triglycerides. The triglyceride component of fats is hydrolysable and consists of three fatty acid molecules that are esterified to glycerol. Therefore, fats are efficient energy substrates and fuel stores in the body. Fats are also important for insulating internal body organs, for maintaining normal core body temperature and for the absorption and storage of fat-soluble vitamins such as Vitamins A, D, E and K. The types of fatty acids that constitute a triglyceride are important for determining its functional and physiological properties [1].

Fatty acids consist of chains of hydrophobic hydrocarbons with a terminal carboxylic acid functional group. Fatty acids can be essential or non-essential. Essential fatty acids cannot be synthesized endogenously and must be consumed via the food. Linoleic and linolenic acids are essential fatty acids. Almost all other fatty acids can be synthesized endogenously from linoleic and linolenic acids or from non-fatty acid precursors and are therefore non-essential [2,3]. Fatty acids are also categorised as saturated or unsaturated. Saturated fatty acids do not contain a double bond because all carbon atoms in the chain are fully bonded to hydrogen atoms. Palmitic and stearic acids are common saturated fatty acids and are found mostly in meat, butter, lard, and palm oil. Unsaturated fatty acids possess one or more double bonds and are mostly from plant sources. Unsaturated fatty acids with only one double bond are distinguished as mono-unsaturated fatty acids (MUFAs). Oleic acid is the most commonly consumed MUFA and it is rich in olive oil, peanut oil, avocado and other nut-based oils. The presence of two or more double bonds categorizes an unsaturated fatty acid as a poly-unsaturated fatty acid (PUFA). PUFAs can further be divided into omega- 3 or omega- 6 depending on the position of the double bond relative to the last (omega) carbon at the methyl end. Alpha-linolenic acid (ALA) is an omega-3 PUFA contained richly in linseed and hempseed oils, whereas eicosapentaenoic acid (EPA) and docosahexaenoic acid (DHA) are omega-3 PUFAs found in microalgae and fish. Linoleic and arachidonic acids are examples of omega- 6 PUFAs and are common in poultry, cereals and nuts. These MUFAs and PUFAs belong to the more common cis-unsaturated fatty acids because the hydrogen atoms around all double bonds point in the same plane. However, less abundant trans-unsaturated fatty acids also exist. In trans fatty acids, the hydrogen atoms that surround one or more of the double bonds point in opposite directions $[1,4]$. 
In general, most MUFAs and PUFAs are known to have beneficial effects on human health but saturated and trans fatty acids have been implicated in several human diseases such as obesity, insulin resistance, diabetes, cardiovascular diseases and cancer [5-7].

\section{Trans fatty acids}

Trans fat is a term that is used to describe triglycerides that are rich in trans fatty acids. Trans fatty acids are unsaturated fatty acids that contain at least one double bond surrounded by trans-configurated hydrogen atoms. Trans fatty acids are less abundant in human diets compared to their cis isoforms.

The artificial synthesis of trans fatty acids became commercialized after Dr Peter Normann patented its industrial production over a century ago [8]. Such artificial trans fatty acids are products of partial hydrogenation of vegetable oils in the presence of a chemical catalyst. This industrial process converts liquid PUFA-rich vegetable oil into solid trans fatty acid-rich fats. Foods that are high in trans fatty acids have the benefit of easier packaging, improved texture, improved taste and increased shelf life. Food products such as margarine, shortenings, crackers, cookies, and deep fried foods are all rich in industrial trans fatty acids [9]. Industrial production aside, relatively small amounts of trans fatty acids are produced by intestinal microbes of ruminants. These trans fatty acids are often referred to as natural or ruminant trans fatty acids and are present in the meat and dairy products of these animals. Elaidic acid is the most abundant industrial trans fatty acid in the diet and represents about $80 \%$ of total trans fatty acids consumed by humans, whereas trans-vaccenic acid is the most abundant natural trans fatty acid $[9,10]$.

A number of epidemiological and clinical studies have shown a strong association between trans fatty acid intake and coronary heart disease [11-13]. It is now known that a $2 \%$ increase in energy resulting from trans fatty acid consumption increases the risk of heart disease by $23 \%$ [14]. Based on these findings, Denmark was the first country to ban the consumption of foods that contain industrial trans fatty acids [15]. Today many other countries have laws that restrict or completely ban food companies from incorporating trans fatty acids into food products [16-20]. Across Europe, the European Union (EU) never took any legislative action in order to remove trans fats from the food supply. Instead, the European food industries voluntarily committed themselves to reduce the trans fatty acid content of foods to a maximum of $2 \%$ of the total fat content. While the overall trans fatty acid content of foods has dropped, the lack of legislation has prevented many food manufacturers, mostly concentrated in Eastern Europe, from 
taking action. The residual presence of trans fat in the EU food supply should drive legislators to consider additional public health approaches to further reduce trans fat intake in Europe, including imposing legal limits on trans fat content in foodstuffs, mandatory and voluntary trans fat labelling, and voluntary food reformulation pledges. In October 2018, the EU solicited input on a new proposal that would set a maximum limit of $2 \%$ for trans fat content in foodstuffs. Food which does not comply to the new regulation may continue to be placed on the market until April 1 2021.These efforts have resulted in a substantial decline in total trans fatty acid intake [21]. However, many other countries do not have such policies or regulations against trans fat consumption. Additionally, a complete elimination of dietary trans fat is impossible due to existence of natural trans fatty acids.

\section{Trans fat and cholesterol metabolism}

Cholesterol is a lipophilic sterol biomolecule that forms a structural component of animal cell membranes and is important for maintaining cell fluidity, division, survival, and function. It is also a precursor for the synthesis of steroid hormones, bile acids, and vitamin D [22]. Notwithstanding its importance, an excessive amount of cholesterol in plasma and tissues is implicated in cardiovascular diseases and must therefore be tightly regulated [23]. Cholesterol is obtained from exogenous and endogenous sources. Exogenous cholesterol is obtained from the diet, absorbed through the small intestine, and packaged into chylomicron particles for onward secretion. Endogenous cholesterol is synthesized mainly in the liver, packaged into lipoprotein particles (VLDL) and secreted into the circulation [24]. Circulating VLDL gives rise to LDL after removal of apolipoproteins $\mathrm{C}$ and $\mathrm{E}$, and hydrolysis of the triglyceride content of VLDL by lipoprotein lipase and hepatic lipase. The resulting LDL particles composed mainly of apolipoprotein B (apoB), cholesterol and cholesteryl esters are the major determinant of plasma cholesterol levels $[25,26]$. Through reverse cholesterol transport, circulating cholesterol that are contained in HDL particles are taken up by the liver for bile acid synthesis or excretion through faeces [27]. Clinical studies have shown that elevated levels of non-HDL cholesterol or apoB-containing lipoproteins such as LDL, VLDL and their remnant particles are associated with higher risk of cardiovascular diseases [28,29].

In the early 1990s, a ground-breaking study conducted in Wageningen showed that industrial trans fatty acids significantly increase plasma levels of total and LDL cholesterol but decrease levels of HDL cholesterol in healthy individuals [30-32]. This raised serious concerns about the safety of dietary trans fatty acids. In the same study, saturated fatty acids also increased levels of LDL cholesterol but showed no effect on 
HDL cholesterol compared to cis-unsaturated fatty acids [30]. This led to the conclusion that in comparison to saturated fatty acids, industrial trans fatty acids likely have stronger deleterious consequence on cardiovascular health. Subsequently, a number of clinical and pre-clinical studies have attempted to investigate the underlying mechanisms for the reported harmful effects of trans fatty acids [33-36]. However, it is still unclear which molecular and cellular processes mediate the effects of trans fatty acids. This has fuelled our research interest to investigate the molecular mechanisms of trans fatty acids.

\section{Transcriptional regulators of cellular lipid metabolism}

A number of transcription factors have been studied for their role in cellular lipid metabolism. These transcriptional factors include peroxisome proliferator-activated receptors (PPARs), sterol regulatory element binding proteins (SREBPs), liver X receptor (LXR), carbohydrate-responsive element-binding protein (ChREBP), farnesoid $X$ receptor (FXR) and NF-E2-related factor-2 (NRF2) [37].

In this thesis, we found that industrial trans fatty acids regulate the transcriptional activity of SREBP2. Also, we purposely investigated the response of Angptl4-deficient mice to diets rich in industrial trans fatty acids. Since ANGPTL4 is direct target of PPARs, the focus in the subsequent sections will be on SREBP and PPARs for their direct relevance to the research contained in this thesis.

\section{Sterol regulatory element binding proteins (SREBPs)}

SREBPs are transcription factors that regulate lipid metabolism through transcriptional regulation of genes involved in fatty acid and cholesterol synthesis. Three isoforms of SREBPs have been identified and characterised: SREBP1a, SREBP1c and SREBP2 [3840]. All three isoforms of SREBP are localised intracellularly in the endoplasmic reticulum membrane as inactive precursors. SREBP1c is much strongly activated by anabolic hormones such as insulin, while SREBP2 activation is strongly induced by low levels of intracellular cholesterol [41]. Therefore SREBP2 preferentially induces the expression of genes for cholesterol synthesis, SREPB1c prominently upregulates the expression of fatty acid synthesis genes whereas SREBP1a is less-specific towards inducing either cholesterol or fatty acid synthesis genes [42,43]. In response to low levels of cholesterol, the sterol sensor protein, SREBP-cleavage activating protein (SCAP) initiates activation of SREBPs by transporting SREBPs to the Golgi apparatus. In the Golgi apparatus, SREBP is cleaved sequentially by Site- 1 and Site- 2 proteases to produce the active forms. The active SREBP enters the nucleus and binds to the specific loci called SREBP-response element (SRE) that are located at the promoter regions of 
target genes. This results in transcriptional activation and mRNA expression of SREBP target genes involved in cholesterol and fatty acid synthesis [42,43]. Typically, SREBP activation promotes mRNA expression of cholesterol biosynthesis genes such as HMGCoA reductase (HMGCR), HMG-CoA synthase (HMGCS), and mevalonate kinase (MVK), as well fatty acid biosynthesis genes such as fatty acid synthase (FASN), ATPcitrate lyase (ACYL) and acetyl-CoA carboxylase (ACC) [44,45].

\section{Peroxisome proliferator activated receptors (PPARs)}

PPARs are nuclear receptors and serve as transcriptional regulators of genes involved in fatty acid metabolism. They are activated in response to binding specific ligands. PPARs exist in 3 isoforms; PPAR-alpha (PPAR $\alpha$ ), PPAR-gamma (PPAR $\gamma$ ) and PPARbeta/delta $(\operatorname{PPAR} \beta / \delta)$. PPAR $\alpha$ is strongly expressed in the liver, but also in the brown adipose tissue. PPAR $\gamma$ is most highly expressed in both white and brown adipose tissue, large intestines and in macrophages, while PPAR $\delta$ is strongly expressed in skeletal and cardiac muscles as well as many other tissues [46-48]. Activation of PPARs can be achieved nutritionally through fatty acids, physiologically through fasting, and pharmacologically through high-affinity agonists such as fenofibrate, Wy14643 and rosiglitazone [49-51]. Activated PPARs form a heterodimer with the Retinoid-X receptors in order to bind to a unique sequence called PPAR response element (PPRE) located in or around target genes [52,53]. The target genes of PPARs are involved in numerous biological pathways related to nutrient and energy metabolism $[54,55]$. Specifically, several target genes of PPAR are known to be directly involved in lipid metabolism, one of which encodes Angiopoietin-like 4 (ANGPTL4) [47,56].

\section{Angiopoietin-like 4 (ANGPTL4)}

ANGPTL4 is a member of the angiopoietin-like family of proteins that includes seven additional members numbering ANGPTL1 to 8 . The Angptl4 gene encodes a protein of 406 amino acids that is approximately $50 \mathrm{kDa}$ in size. ANGPTL4 was discovered in 2000 as a target gene of PPAR following a fasting study in PPAR $\alpha$-null and wild-type mice and was therefore named as fasting-induced adipose factor (FIAF) [57]. The ANGPTL4 gene consists of 7 exons and 6 introns, with a PPAR-response element (PPRE) located within intron 3 [58]. ANGPTL4 is ubiquitously expressed in the adipose tissue, liver, kidney, intestine, cardiac and skeletal muscles, as well as in macrophages [56,57,59]. The ANGPLT4 protein is made up of an N-terminal coiled-coiled domain and a Cterminal fibrinogen-like domain that are connected by a linker region. It is well established that ANGPTL4 has a major impact on lipid metabolism via its $\mathrm{N}$-terminal 
domain. Less is known about the role of C-terminal portion of ANGPTL4. A number of studies have shown that C-terminal or full length ANGPLT4 is involved in various biological processes such as angiogenesis [60,61], wound healing [62], tumorigenesis [63-65] and kidney function [66].

The N-terminal domain of ANGPLT4 directly regulates circulating levels of plasma triglycerides by inhibiting the enzyme lipoprotein lipase (LPL) [67-69]. LPL functions as an extracellular lipase that hydrolyses triglycerides contained in circulating triglyceride-rich lipoproteins such as chylomicrons and VLDL. The resultant free fatty acids are taken up by the underlying tissues for storage or as energy substrates [70,71]. Non-functional LPL due to inhibition by ANGPLT4 results in increased levels of circulating triglycerides. Therefore, ANGPTL4 plays an important role in maintaining systemic triglyceride homeostasis under different physiological, nutritional, and clinical conditions [72,73]. In humans, a loss-of-function mutation in ANGPTL4 leads to a reduction in levels of plasma triglycerides with an associated reduced odds of developing coronary artery disease $[74,75]$. Therefore, current research is investigating the potential targeting of ANGPTL4 as a strategy against dyslipidaemia and associated cardiovascular diseases.

\section{Outline of thesis}

The research contained in this thesis has two overarching aims: (1) to probe the mechanisms of cellular lipid metabolism with a focus on trans fatty acids and (2) to further characterize the lipid-sensitive ANGPTL4 protein for its role in lipid metabolism and the potential of targeting ANGPTL4 for correcting cardiometabolic disorders. Chapter $\mathbf{1}$ is a general introduction and explanation of the key terms and concepts of this thesis. Chapter 2 presents a contemporary review of existing literature on the molecular effects of trans fatty acids. In Chapter 3, we have compared the physiological response of mice to diets enriched in either trans-unsaturated, cis-unsaturated or saturated fatty acids. At the molecular level, we investigated the effects of elaidate, oleate and palmitate as representatives of trans-unsaturated, cis-unsaturated and saturated fatty acids, respectively, using murine and human cell lines. In Chapter 4, we have studied the response of Angptl4 ${ }^{-/}$mice to the different types of dietary fat as used in Chapter 3. We were specifically interested in the response of Angptl4 ${ }^{-/}$mice to diets rich in trans fatty acids. We complemented the mice findings with in vitro experiments in murine macrophages. In Chapter $\mathbf{5}$ of this thesis, we investigated the clinical potential of targeting ANGPTL4 by studying the effects of fasting and a high saturated fat diet in ANGPTL4-hypomorph mice with complete deletion of the C-terminal domain of 
ANGPTL4, and a partial expression of the N-terminal LPL-inhibiting domain. Additionally, Chapter 5 also investigates the influence of ANGPTL4 on lipid uptake by macrophages. Chapter 6 contains a collaborative research project that investigated the role of muscle specific inflammation on whole body insulin sensitivity in mice. Chapter 7 is a general discussion of the major outcomes of these studies, recommendations for future studies and a final conclusion. 


\section{References}

[1] Tvrzicka, E., Kremmyda, L.S., Stankova, B., Zak, A., 2011. Fatty acids as biocompounds: Their role in human metabolism, health and disease - a review. part 1: Classification, dietary sources and biological functions. Biomedical Papers 155(2): 117-30.

[2] Das, U., 2006. Essential Fatty Acids - A Review. Current Pharmaceutical Biotechnology 7(6): 467-82.

[3] Burr, G O and Burr, M.M., 1930. On the nature and role of the fatty acids essential in nutrition. J Biol Chem 86: 587-620.

[4] Rustan, A.C., Drevon, C.A., 2005. Fatty Acids: Structures and Properties. Encyclopedia of Life Sciences: 1-7.

[5] Sacks, F.M., Lichtenstein, A.H., Wu, J.H.Y., Appel, L.J., Creager, M.A., KrisEtherton, P.M., et al., 2017. Dietary fats and cardiovascular disease: A presidential advisory from the American Heart Association. Circulation 136(3): e1-23.

[6] Willett, W.C., 2012. Dietary fats and coronary heart disease. Journal of Internal Medicine 272(1): 13-24.

[7] Estadella, D., Claudia, M., Oller, P., Piano, A. De., 2014. Lipotoxicity : Effects of Dietary Saturated and Transfatty Acids. Hindawi 2013: 1-15.

[8] Ginter, E., Simko, V., 2016. New data on harmful effects of trans-fatty acids. Bratislavské Lekárske Listy 117(5): 251-3.

[9] Ganguly, R., Pierce, G.N., 2015. The toxicity of dietary trans fats. Food and Chemical Toxicology 78: 170-6.

[10] Stender, S., Astrup, A., Dyerberg, J., 2008. Ruminant and industrially produced trans fatty acids: Health aspects. Food and Nutrition Research 52: 1-8.

[11] Ganguly, R., Pierce, G.N., 2012. Trans fat involvement in cardiovascular disease. Molecular Nutrition and Food Research 56(7): 1090-6.

[12] Bendsen, N.T., Christensen, R., Bartels, E.M., Astrup, A., 2011. Consumption of industrial and ruminant trans fatty acids and risk of coronary heart disease: A systematic review and meta-analysis of cohort studies. European Journal of Clinical Nutrition 65(7): 773-83.

[13] Filip, S., Vidrih, R., 2008. Trans Fatty Acids and Human Health 16.

[14] Mozaffarian, D., Katan, M.B., Ascherio, A., Stampfer, M.J., Willett, W.C., 2006. Trans Fatty Acids and Cardiovascular Disease. New England Journal of Medicine 354(15): 1601-13.

[15] Stender, S., Dyerberg, J., Astrup, A., 2006. Consumer protection through a legislative ban on industrially produced trans fatty acids in foods in Denmark. Scandinavian Journal of Food \& Nutrition 50(July): 155-60. 
[16] Monge-Rojas, R., Colón-Ramos, U., Jacoby, E., Alfaro, T., Tavares do Carmo, M.D.G., Villalpando, S., et al., 2017. Progress towards elimination of trans-fatty acids in foods commonly consumed in four Latin American cities. Public Health Nutrition (12): 1-10.

[17] Pérez-Farinós, N., Dal Re Saavedra, M.Á., Villar Villalba, C., Robledo de Dios, T., 2016. Trans-fatty acid content of food products in Spain in 2015. Gaceta Sanitaria 30(5): 379-82.

[18] Roe, M., Pinchen, H., Church, S., Elahi, S., Walker, M., Farron-Wilson, M., et al., 2013. Trans fatty acids in a range of UK processed foods. Food Chemistry 140(3): 427-31.

[19] Kaur, G., Cameron-Smith, D., Sinclair, A.J., 2012. Are trans fats a problem in Australia? The Medical Journal of Australia 196(11): 666-7.

[20] Craig-Schmidt, M.C., 2006. World-wide consumption of trans fatty acids. Atherosclerosis Supplements 7(2): 1-4.

[21] Downs, S.M., Bloem, M.Z., Zheng, M., Catterall, E., Thomas, B., Veerman, L., et al., 2017. The Impact of Policies to Reduce trans Fat Consumption: A Systematic Review of the Evidence. Current Developments in Nutrition 1(12).

[22] Vance, D.E., Van Den Bosch, H., 2000. Cholesterol in the year 2000. Biochimica et Biophysica Acta - Molecular and Cell Biology of Lipids 1529(1-3): 1-8.

[23] SATO, R., 2015. Functions of Cholesterol Metabolites. Journal of Nutritional Science and Vitaminology 61(Supplement): S151-3.

[24] Sheperd, J., 2001. The Role of the Exagenous Pathway in Hypercholesterolaemia. European Heart Journal 3: 2-5.

[25] Sacks, F.M., Campos, H., 2003. Cardiovascular endocrinology 4: Low-Density Lipoprotein Size and Cardiovascular Disease: A Reappraisal. Journal of Clinical Endocrinology and Metabolism 88(10): 4525-32.

[26] Cox, R.A., García-Palmieri, M.R., 1990. Cholesterol, Triglycerides, and Associated Lipoproteins. Clinical Methods: The History, Physical, and Laboratory Examinations.

[27] Temel, R.E., Brown, J.M., 2015. A new model of reverse cholesterol transport: EnTICEing strategies to stimulate intestinal cholesterol excretion. Trends in Pharmacological Sciences 36(7): 440-51.

[28] Chan, D.C., Watts, G.F., 2006. Apolipoproteins as markers and managers of coronary risk. QJM - Monthly Journal of the Association of Physicians 99(5): 27787.

[29] Ramasamy, I., 2014. Recent advances in physiological lipoprotein metabolism. Clinical Chemistry and Laboratory Medicine 52(12): 1695-727.

[30] Mensink, R.P.\&., Katan, M.B., 1990. Effect of dietary trans fatty acids on highdensity and low-density lipoprotein cholesterol levels in healthy subjects. New 
England Journal of Medicine 327(2): 82-7.

[31] Zock, P.L., Katan, M.B., 1992. Hydrogenation alternatives: effects of trans fatty acids and stearic acid versus linoleic acid on serum lipids and lipoproteins in humans. Journal of Lipid Research 33(3): 399-410.

[32] Katan, M.B., Zock, P.L., Mensink, R.P., 1995. Trans fatty acids and their effects on lipoproteins in humans. Annual Review of Nutrition 15: 473-93, Doi: 10.1146/annurev.nutr.15.1.473.

[33] Matthan, N.R., Welty, F.K., Barrett, P.H.R., Harausz, C., Dolnikowski, G.G., Parks, J.S., et al., 2004. Dietary hydrogenated fat increases high-density lipoprotein apoAI catabolism and decreases low-density lipoprotein apoB-100 catabolism in hypercholesterolemic women. Arteriosclerosis, Thrombosis, and Vascular Biology 24(6): 1092-7.

[34] Mauger, J.F., Lichtenstein, A.H., Ausman, L.M., Jalbert, S.M., Jauhiainen, M., Ehnholm, C., et al., 2003. Effect of different forms of dietary hydrogenated fats on LDL particle size. American Journal of Clinical Nutrition 78(3): 370-5.

[35] Monguchi, T., Hara, T., Hasokawa, M., Nakajima, H., Mori, K., Toh, R., et al., 2017. Excessive intake of trans fatty acid accelerates atherosclerosis through promoting inflammation and oxidative stress in a mouse model of hyperlipidemia. Journal of Cardiology 70(2): 121-7.

[36] Vendel Nielsen, L., Krogager, T.P., Young, C., Ferreri, C., Chatgilialoglu, C., Nørregaard Jensen, O., et al., 2013. Effects of Elaidic Acid on Lipid Metabolism in HepG2 Cells, Investigated by an Integrated Approach of Lipidomics, Transcriptomics and Proteomics. PLoS ONE 8(9).

[37] Georgiadi, A., Kersten, S., 2012. Mechanisms of Gene Regulation by Fatty Acids. Advances in Nutrition 3(2): 127-34.

[38] Hua, X., Wu, J., Goldstein, J.L., Brown, M.S., Hobbs, H.H., 1995. Structure of the human gene encoding sterol regulatory element binding protein-1 (SREBF1) and localization of SREBF1 and SREBF2 to chromosomes 17p11.2 and 22q13. Genomics 25(3): 667-73.

[39] Brown, M., Goldstein, J., 1997. SREBP pathway: regulation of cholesterol metabolism by proteolysis of membrane bound transcription factor. Cell 89(1): 33140.

[40] Shimomura, I., Shimano, H., Horton, J.D., Goldstein, J.L., Brown, M.S., 1997. Differential expression of exons $1 \mathrm{a}$ an $1 \mathrm{c}$ in mRNAs for sterol regulatory element binding protein 1 in human and mouse organs and cultured cells. J. Clin. Invest. 99: 838-45.

[41] DeBose-Boyd, R.A., Ye, J., 2018. SREBPs in Lipid Metabolism, Insulin Signaling, and Beyond. Trends in Biochemical Sciences 43(5): 358-68.

[42] DeBose-Boyd, R.A., Ye, J., 2011. Regulation of Cholesterol and Fatty Acid Synthesis. Cold Spring Harbor Perspectives in Biology 3(7): 1-14. 
[43] Horton, J.D., Goldstein, J.L., Brown, M.S., 2002. Critical review. Most 109(9): 1125-31.

[44] Rome, S., Lecomte, V., Meugnier, E., Rieusset, J., Debard, C., Euthine, V., et al., 2008. Microarray analyses of SREBP-1a and SREBP-1c target genes identify new regulatory pathways in muscle. Physiological Genomics 34(3): 327-37.

[45] Amemiya-Kudo, M., Shimano, H., Hasty, A.H., Yahagi, N., Yoshikawa, T., Matsuzaka, T., et al., 2002. Transcriptional activities of nuclear SREBP-1a, -1c, and -2 to different target promoters of lipogenic and cholesterogenic genes. Journal of Lipid Research 43(8): 1220-35.

[46] Bookout, A.L., Jeong, Y., Downes, M., Yu, R.T., Evans, R.M., Mangelsdorf, D.J., 2006. Anatomical Profiling of Nuclear Receptor Expression Reveals a Hierarchical Transcriptional Network. Cell 126(4): 789-99.

[47] Kersten, S., 2014. Integrated physiology and systems biology of PPAR $\alpha$. Molecular Metabolism 3(4): 354-71.

[48] Kersten, S., Stienstra, R., 2017. The Role and Regulation of the Peroxisome Proliferator Activated Receptor alpha in Human Liver. Biochimie 136: 75-84.

[49] Kersten, S., Seydoux, J., Peters, J.M., Gonzalez, F.J., Desvergne, B., Wahli, W., 1999. Peroxisome proliferator-activated receptor $\alpha$ mediates the adaptive response to fasting. Journal of Clinical Investigation 103(11): 1489-98.

[50] Forman, BM, Chen, J, Evans, R., 1997. Hypolipidemic drugs, polyunsaturated fatty acids, and eicosanoids are ligands for peroxisome proliferator-activated receptors $\alpha$ and $\delta$. Pnas 94(April): 4312-7.

[51] Sanderson, L.M., Degenhardt, T., Koppen, A., Kalkhoven, E., Desvergne, B., Muller, M., et al., 2009. Peroxisome Proliferator-Activated Receptor / (PPAR /) but Not PPAR Serves as a Plasma Free Fatty Acid Sensor in Liver. Molecular and Cellular Biology 29(23): 6257-67.

[52] DiRenzo, J., Söderstrom, M., Kurokawa, R., Ogliastro, M.H., Ricote, M., Ingrey, S., et al., 1997. Peroxisome proliferator-activated receptors and retinoic acid receptors differentially control the interactions of retinoid $\mathrm{X}$ receptor heterodimers with ligands, coactivators, and corepressors. Molecular and Cellular Biology 17(4): 2166-76.

[53] Tugwood, J.D., Issemann, I., Anderson, R.G., Bundell, K.R., McPheat, W.L., Green, S., 1992. The mouse peroxisome proliferator activated receptor recognizes a response element in the 5 ' flanking sequence of the rat acyl CoA oxidase gene. The EMBO Journal 11(2): 433-9.

[54] Rakhshandehroo M., Knoch B., Muller M., K.S., 2004. Peroxisome proliferatoractivated receptor $\alpha$ target genes. Cellular and Molecular Life Sciences 61(4): 393 416.

[55] Tachibana, K., Kobayashi, Y., Tanaka, T., Tagami, M., Sugiyama, A., Katayama, T., et al., 2005. Gene expression profiling of potential peroxisome proliferator- 
activated receptor (PPAR) target genes in human hepatoblastoma cell lines inducibly expressing PPAR isoforms. Nuclear Receptor 3: 1-17.

[56] Zhu, P., Goh, Y.Y., Chin, H.F.A., Kersten, S., Tan, N.S., 2012. Angiopoietin-like 4: a decade of research. Bioscience Reports.

[57] Kersten, S., Mandard, S., Tan, N.S., Escher, P., Metzger, D., Chambon, P., et al., 2000. Characterization of the fasting-induced adipose factor FIAF, a novel peroxisome proliferator-activated receptor target gene. Journal of Biological Chemistry 275(37): 28488-93.

[58] Mandard, S., Zandbergen, F., Nguan, S.T., Escher, P., Patsouris, D., Koenig, W., et al., 2004. The direct peroxisome proliferator-activated receptor target fastinginduced adipose factor (FIAF/PGAR/ANGPTL4) is present in blood plasma as a truncated protein that is increased by fenofibrate treatment. Journal of Biological Chemistry 279(33): 34411-20.

[59] Yoon, J.C., Chickering, T.W., Rosen, E.D., Dussault, B., Qin, Y., Soukas, A., et al., 2000. Peroxisome proliferator-activated receptor gamma target gene encoding a novel angiopoietin-related protein associated with adipose differentiation. Mol. Cell. Biol. 20(14): 5343-9.

[60] Perdiguero, E.G., Galaup, A., Durand, M., Teillon, J., Philippe, J., Valenzuela, D.M., et al., 2011. Alteration of developmental and pathological retinal angiogenesis in angptl4-deficient mice. Journal of Biological Chemistry 286(42): 36841-51.

[61] Ito, Y., Oike, Y., Yasunaga, K., Hamada, K., Miyata, K., Matsumoto, S.I., et al., 2003. Inhibition of Angiogenesis and Vascular Leakiness by Angiopoietin-Related Protein 4. Cancer Research 63(20): 6651-7.

[62] Pal, M., Tan, M.J., Huang, R.L., Goh, Y.Y., Wang, X.L., Tang, M.B.Y., et al., 2011. Angiopoietin-like 4 regulates epidermal differentiation. PLoS ONE 6(9).

[63] Toshiyuki, N., Hiroshi, H., Kenichiro, S., Arifa, N., Kuniko, A., Takeshi, N.A., et al., 2011. Expression of angiopoietin-like 4 (ANGPTL4) in human colorectal cancer: ANGPTL4 promotes venous invasion and distant metastasis. Oncology Reports 25(4): 929-35.

[64] Tan, Z.W., Teo, Z., Tan, C., Choo, C.C., Loo, W.S., Song, Y., et al., 2017. ANGPTL4 T266M variant is associated with reduced cancer invasiveness. Biochimica et Biophysica Acta - Molecular Cell Research 1864(10): 1525-36.

[65] Tan, M.J., Teo, Z., Sng, M.K., Zhu, P., Tan, N.S., 2012. Emerging Roles of Angiopoietin-like 4 in Human Cancer. Molecular Cancer Research 10(6): 677-88.

[66] Clement, L.C., Avila-Casado, C., MacÉ, C., Soria, E., Bakker, W.W., Kersten, S., et al., 2011. Podocyte-secreted angiopoietin-like-4 mediates proteinuria in glucocorticoid-sensitive nephrotic syndrome. Nature Medicine 17(1): 117-22.

[67] Sukonina, V., Lookene, A., Olivecrona, T., Olivecrona, G., 2006. Angiopoietin-like protein 4 converts lipoprotein lipase to inactive monomers and modulates lipase activity in adipose tissue. Proceedings of the National Academy of Sciences. 
[68] Dijk, W., Beigneux, A.P., Larsson, M., Bensadoun, A., Young, S.G., Kersten, S., 2016. Angiopoietin-like 4 (ANGPTL4) promotes intracellular degradation of lipoprotein lipase in adipocytes. Journal of Lipid Research 7(11): 956-63.

[69] Dijk, W., Ruppert, P.M.M., Oost, L.J., Kersten, S., 2018. Angiopoietin-like 4 promotes the intracellular cleavage of lipoprotein lipase by PCSK3/furin in adipocytes. Journal of Biological Chemistry 293(36): 14134-45.

[70] Merkel M, Eckel, R. and G.I., 2002. Lipoprotein lipase: genetics, lipid uptake, and regulation. J. Lipid Res.: 1997-2006.

[71] Kersten, S., 2014. Physiological regulation of lipoprotein lipase. Biochimica et Biophysica Acta 1841(7): 919-33.

[72] Dijk, W., Kersten, S., 2014. Regulation of lipoprotein lipase by Angpt14. Trends in Endocrinology and Metabolism 25(3): 146-55.

[73] Mattijssen, F., Kersten, S., 2012. Regulation of triglyceride metabolism by Angiopoietin-like proteins. Biochimica et Biophysica Acta 1821(5): 782-9.

[74] Dewey, F.E., Gusarova, V., O’Dushlaine, C., Gottesman, O., Trejos, J., Hunt, C., et al., 2016. Inactivating Variants in ANGPTL4 and Risk of Coronary Artery Disease. New England Journal of Medicine 374(12): 1123-33.

[75] Abid, K., Trimeche, T., Mili, D., Msolli, M.A., Trabelsi, I., Nouira, S., et al., 2016. ANGPTL4 variants E40K and T266M are associated with lower fasting triglyceride levels and predicts cardiovascular disease risk in Type 2 diabetic Tunisian population. Lipids in Health and Disease 15(1): 63. 
Chapter 2 


\title{
Molecular effects of trans fatty acids
}

\author{
Antwi-Boasiako Oteng \& Sander Kersten
}




\section{Abstract}

Studies in humans have established a positive association between intake of industrial trans fatty acids and the development of cardiovascular diseases. The strength of the association is such that several countries have enacted laws that restrict the incorporation of industrial trans fatty acids in food products. However, trans fatty acids cannot be completely eliminated from the human diet since they are also naturally present in meat and dairy products of ruminant animals. Moreover, bans on industrial trans fatty acids have not yet been instituted in all countries. The epidemiological and clinical evidence against trans fatty acids by far overshadows mechanistic and molecular insights that may explain how these trans fatty acids achieve their damaging effects. In this review, the cellular and molecular studies on trans fatty acids are compiled in an attempt to improve our understanding of the molecular mechanisms that underlie their deleterious effects. In doing so, effects of trans fatty acids will be juxtaposed against those of cis-unsaturated and saturated fatty acids. This review will also carefully explore the argumentation that natural trans fatty acids from ruminants have contrasting effects to artificial trans fatty acids from industrial sources. Overall, a number of in vivo and in vitro studies demonstrate that industrial trans fatty acids promote inflammation and ER stress although to a much lower extent compared to saturated fatty acids, whereas cisunsaturated fatty acids are protective against ER stress and inflammation. Also, industrial trans fatty acids promote storage of fat in the liver at the expense of fat tissue compared to cis-unsaturated and saturated fatty acids. In cultured liver and adipocyte cells, industrial trans fatty acids but not cis-unsaturated or saturated fatty acids stimulate the cholesterol synthesis pathway by activating SREBP2-mediated gene regulation. The effects of trans fatty acids on inflammation, ER stress and cholesterogenesis appear to be specific to industrial but not ruminant trans fatty acids. Clearer insight into the molecular mechanisms of action of trans fatty acids may create new therapeutic windows for the treatment of diseases characterised by disrupted lipid metabolism. 


\section{Introduction}

Trans fatty acids are unsaturated fatty acids that contain one or more unconjugated double bond in the trans configuration. The term trans fats is used to describe triglycerides that are rich in trans fatty acids. The majority of trans fatty acids are generated during industrial processing through partial hydrogenation of vegetable oils rich in poly-unsaturated fatty acids. Foods containing these industrially produced artificial trans fatty acids carry several benefits including improved texture, better taste, and enhanced longevity [1-3]. The enhanced shelf life prompted heightened use and consumption of trans fatty acids during the first and second world wars, when food was in short supply. In 1957, twelve years after World War II, a study by Dr. Fred Kummerow showed that lipid extracts of specimens from 24 human subjects who died of heart disease contained up to $12.2 \%$ trans fatty acids in their adipose tissue, $14.4 \%$ in liver, $9.3 \%$ in heart tissue, $8.8 \%$ in aortic tissue and $8.8 \%$ in atheroma [4]. Subsequent studies such as conducted by Thomas and colleagues showed that the level of trans fatty acids in adipose tissue of persons who died of coronary heart disease was significantly higher compared to persons who died of other causes [5,6]. In 1990, Mensink and Katan firmly demonstrated the plasma cholesterol-raising effect of trans fatty acids in human volunteers [7]. Several years later, Willett and colleagues found that in the Nurses' health study, after adjustment for age and total energy intake, intake of trans fatty acids was positively associated with risk of coronary heart disease [8]. Also, in a case-control study, Thomas had found that in the fat tissue of subjects who died of ischemic heart disease, the content of trans palmitoleic acid was significantly higher compared with the fat tissue of subjects who died of unrelated causes [9]. The studies that strongly connected the intake of trans fatty acids with cardiovascular derailment were initially abhorred by players in the margarine industry. However, this later fuelled more research into the pro-atherogenicity of trans fatty acids. Collectively, these studies strongly suggest a causal relationship between trans fatty acid consumption and the development cardiovascular diseases in humans [8,10-14]. In response to this finding, a number of countries enacted laws that either restrict or completely ban food companies from incorporating trans fatty acids into their food products. Whereas in many countries food items such as margarine, crackers, bakery products, cookies and deep fried foods were previously loaded with industrial trans fatty acids, nowadays the levels of trans fatty acids in these foods are very low, which has resulted in a substantial decline in the intake of industrial trans fatty acids in many countries [15-24]. However, trans fatty acids cannot be completely removed from human diets as ruminant trans fatty acids exist. 
These trans fatty acids, which include vaccenic acid and rumenic acid, are generated through bio-hydrogenation of poly-unsaturated fatty acids in the guts of ruminant animals and are therefore present in meats and dairy products [25-27]. In addition, industrial trans fatty acids persist in our food supply because laws aimed at banning industrial trans fatty acids have not yet been instituted in every country.

Because the health concerns connected to the consumption of industrial trans fatty acids could be mitigated by removing them from foods, there has been little incentive for basic researchers to probe mechanistic insights related to trans fatty acids. The end result is a marked imbalance between the vast amount of epidemiological data on trans fatty acids and health outcomes and the limited understanding of the molecular mode of action of trans fatty acids. By collating the cellular and molecular studies on trans fatty acids, this review aims to improve our understanding of the molecular mechanisms underlying the deleterious effects of trans fatty acids. To achieve this objective, some parallels will be drawn between the literature on trans fatty acids and other relevant types of fatty acids. In addition, this review will carefully dissect the argumentation that ruminant trans fatty acids have contrasting effects to artificial trans fatty acids produced during industrial processing.

\section{Chemistry of trans fatty acids}

Trans fatty acids are defined by the presence of one or more trans unconjugated double bonds in their aliphatic hydrocarbon chain. In the trans configuration, the two hydrogen atoms around the double bond point towards opposite planes, whereas in the cis configuration, these hydrogen atoms point in same direction. In comparison to the cis form, the trans double bond produces a more extended fatty acid-carbon chain due to greater bond angle, giving rise to a straight chain tertiary structure akin to that of saturated fatty acids (Figure 1) [2,28]. Differences in tertiary structure affect crystalline packaging, which in turn influences physicochemical properties such as the melting point. For example, the 18-carbon cis oleic acid is liquid at room temperature, with a melting point of $14{ }^{\circ} \mathrm{C}$. By contrast, elaidic acid, which is the trans geometric isomer of oleic acid, has a much higher melting point at $45^{\circ} \mathrm{C}$ and is solid at room temperature. For comparison, the fully saturated stearic acid has a melting pint of $69^{\circ} \mathrm{C}$ [29]. As discussed below, evidence exists to show that beyond affecting their geometric isomerisation and physicochemical properties, the cis-trans configuration of fatty acids has a major influence on the physiological properties after consumption in humans. 
<smiles>CCCCCCCC/C=C/CCCCCCCC(=O)O</smiles>

Trans-unsaturated elaidic acid

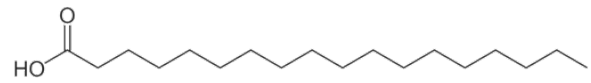

Saturated stearic acid

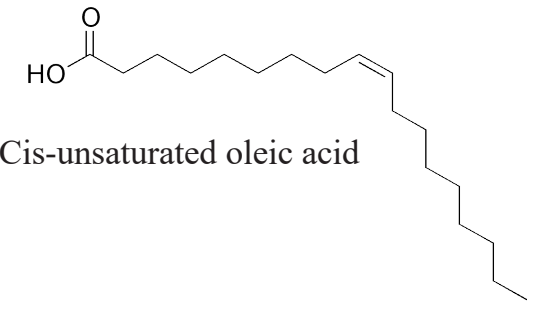

Figure 1. Structure of the geometric isomers of $\mathrm{C} 18$ fatty acids showing elaidic acid with trans double bond, oleic acid with cis double bond and fully saturated stearic acid.

\section{Trans fatty acid effect on molecular players of heart disease}

Although the majority of studies performed on trans fatty acids are observational, a substantial number of experimental studies have been performed. These studies vary from studies in cultured cells and animal models to human clinical trials. A number of these studies have provided evidence that certain trans fatty acids influence the regulation of physiological processes such as lipid metabolism, inflammation, oxidative stress, endoplasmic reticulum (ER) stress, autophagy, apoptosis and even the gut microbiota. The dysregulation of some of these biological pathways by trans fatty acids has been invoked as a potential underlying mechanism that contributes to the negative effects of trans fatty acids on cardiometabolic health [30-32].

\section{Plasma cholesterol and lipoprotein profile}

Elevated levels of plasma cholesterol are a risk factor for cardiovascular disease. Cholesterol is transported in the blood mainly as part of low-density lipoproteins (LDL) and high-density lipoproteins (HDL). Smaller amounts of cholesterol are also contained in the triglyceride-rich chylomicrons, very low-density lipoproteins (VLDL), and their remnant particles. Increased levels of non-HDL cholesterol or apolipoprotein B (apoB)containing particles are associated with increased risk of cardiovascular diseases [3335].

Several studies have examined the effect of trans fatty acids on plasma cholesterol levels and lipoprotein dynamics. Randomized controlled trials carried out at Wageningen University in the 1990s showed that a diet rich in trans fatty acids from partially hydrogenated sunflower oil significantly increased the serum concentration of total and 
LDL cholesterol but decreased HDL cholesterol in comparison with cis-unsaturated or saturated fatty acid-rich diets [7,36]. In a separate randomized controlled trial conducted in 32 healthy men and women, trans fatty acid intake from partially hydrogenated soybean oil decreased serum HDL cholesterol levels but had no effect on LDL cholesterol and triglyceride levels in comparison with a saturated fat diet [37]. In another study conducted in 50 normocholesterolemic men, intake of a trans fatty acid rich diet significantly increased plasma levels of total and LDL cholesterol with no significant effect on HDL cholesterol and triglyceride levels [38]. In providing some mechanistic insights, Mauger and colleagues have reported that the atherogenicity of trans fatty acids may lie in part in their ability to reduce LDL particle size in a dose-dependent manner [39]. Matthan and colleagues have likewise shown in hypercholesterolemic women that the deleterious lipoprotein profile caused by trans fatty acid intake is partly explained by increased apoA1 and decreased apoB100 catabolism [40]. Interestingly, a limited number of studies did not find a significant deleterious effect of trans fatty acid intake on plasma lipid levels $[41,42]$. These discrepant results are potentially due to the use of a different reference or control diet. Additionally, differences in amounts of trans fatty acids consumed may account for some of the conflicting findings, as the effect of trans fatty acids on plasma lipids seems proportional to intake [43]. Overall, it can be concluded that the positive association between trans fatty acid intake and cardiovascular risk is likely at least partly explained by the unfavorable effect of trans fatty acids on plasma LDL cholesterol and the overall lipoprotein profile.

\section{Inflammation}

Inflammation is characterized by pain, swelling, redness, heat, and loss of function. It is the dominant response of body tissues to various types of harmful stimuli, including radiation, physical stress, and harmful chemicals. In addition, inflammation can be induced by microbial infection and tissue damage and as such is an essential mechanism of the innate immune response $[44,45]$. Sterile inflammation describes inflammation that occurs in the absence of an infection and is a symptom of numerous chronic diseases and pathologies, including atherosclerosis. Indeed, in addition to being a lipid-driven process, atherosclerosis is primarily an inflammatory disease characterized by the accumulation of macrophage foam cells in the vascular wall, triggering the secretion of numerous inflammatory mediators and leading to the recruitment of other immune cells [46]. One mechanism by which trans fatty acids may promote atherogenesis is by activating inflammation [32]. In randomized controlled trials, diets that contained trans fatty acids increased plasma levels of inflammatory markers such as C-reactive protein (CRP), TNF $\alpha$, IL-6, IL-1 $\beta$, and MCP-1 in healthy men [47], in subjects with moderate 
hypercholesterolemia [48], and in overweight women [49]. Furthermore, in a crosssectional study in patients with heart disease, levels of trans fatty acids in red blood cell membranes, which is a marker for intake, were positively associated with several inflammatory markers [50].

Due to ethical constraints and other challenges connected with human studies, mechanistic probing into the pro-inflammatory effects of trans fatty acids has been conducted in animal models and in vitro experiments. Two separate studies in the atherosclerosis-prone LDL-receptor knockout mice shed some light on the molecular properties of trans fatty acids obtained from partially hydrogenated soybean oil. In both studies, the industrial trans fatty acids not only increased plasma levels of total and LDL cholesterol but also increased levels of plasma inflammatory cytokines as well as cytokines released into culture medium from primary macrophages when compared to a PUFA-rich diet [51,52]. Secondary to the induction of inflammation, the mice on the trans fatty acid diet showed increased accumulation of activated macrophages in the enlarged atherosclerotic lesions located in the aortic intima [51,52]. Another animal study reported that ad libitum feeding of C57BL/6 mice with a diet supplemented with partially hydrogenated vegetable oil resulted in a hepatic necro-inflammatory phenotype characterised by a marked increase in hepatic TNF $\alpha$ expression [53]. The proinflammatory effect of trans fatty acids seems robust and transcends specific animal models. In PPAR $\alpha$ deficient mice as well as in mice with full body or liver-specific knockout of 11ß-HSD1, trans fatty acid-enriched diets enhanced activation of NF-kB [54] and increased hepatic expression of Tnfa, Mcpl, osteopontin and macrophage markers [54,55]. At the cellular level, the activation of the transcription factor NF-kB by the industrial trans elaidic and linoelaidic acids but not cis-unsaturated linoleic acid was demonstrated in human microvesicular endothelial cells (Figure 2). In this study, the authors found that the profound activation of NF-kB signalling by industrial trans fatty acids is a consequence of enhanced IkB $\alpha$ phosphorylation, which subsequently leads to elevated levels of IL6 and TNF $\alpha$ [56]. In a recent study, Hirata and colleagues showed that in comparison to both control and oleic acid, elaidic acid stimulated apoptotic cell death in RAW264.7 macrophages through hyperactivation of the apoptosis signalregulating kinase 1 (ASK1)-p38 mitogen-activated protein (MAP) kinase pathway that resulted in increased inflammation [57].

Whereas the above studies point to pro-inflammatory effects of trans fatty acids, a number of other studies have reported neutral or anti-inflammatory effects of certain trans fatty acids. We recently showed in ANGPTL4 deficient C57B1/6 mice that a diet rich in trans fatty acids from partially hydrogenated soybean oil did not induce 
inflammation compared to a diet high in saturated fatty acids. Mice deficient in ANGPTL4 - an endogenous inhibitor of lipoprotein lipase - have a unique phenotype characterized by the development of a massive acute phase response upon consumption of a diet rich in saturated fatty acids. The saturated fatty acid-enriched diet markedly increased plasma levels and hepatic expression of serum amyloid A, haptoglobin and lipocalin, whereas the trans fatty acid-enriched diet did not [58]. Studies in RAW264.7 macrophages showed that certain trans fatty acids such as elaidic acid have a neutral effect or modestly decrease inflammatory markers, whereas saturated fatty acids such as palmitic and stearic acid increase mRNA expression and protein levels of inflammatory and apoptotic markers [58]. Accordingly, whether trans fatty acids should be classified as pro-inflammatory may depend on the reference, e.g. no treatment, cis unsaturated fatty acids, or saturated fatty acids. Notwithstanding the considerations, experimental data from clinical studies, animal and cell culture experiments suggest that the deleterious effects of trans fatty acids may be partly mediated by activating inflammation.

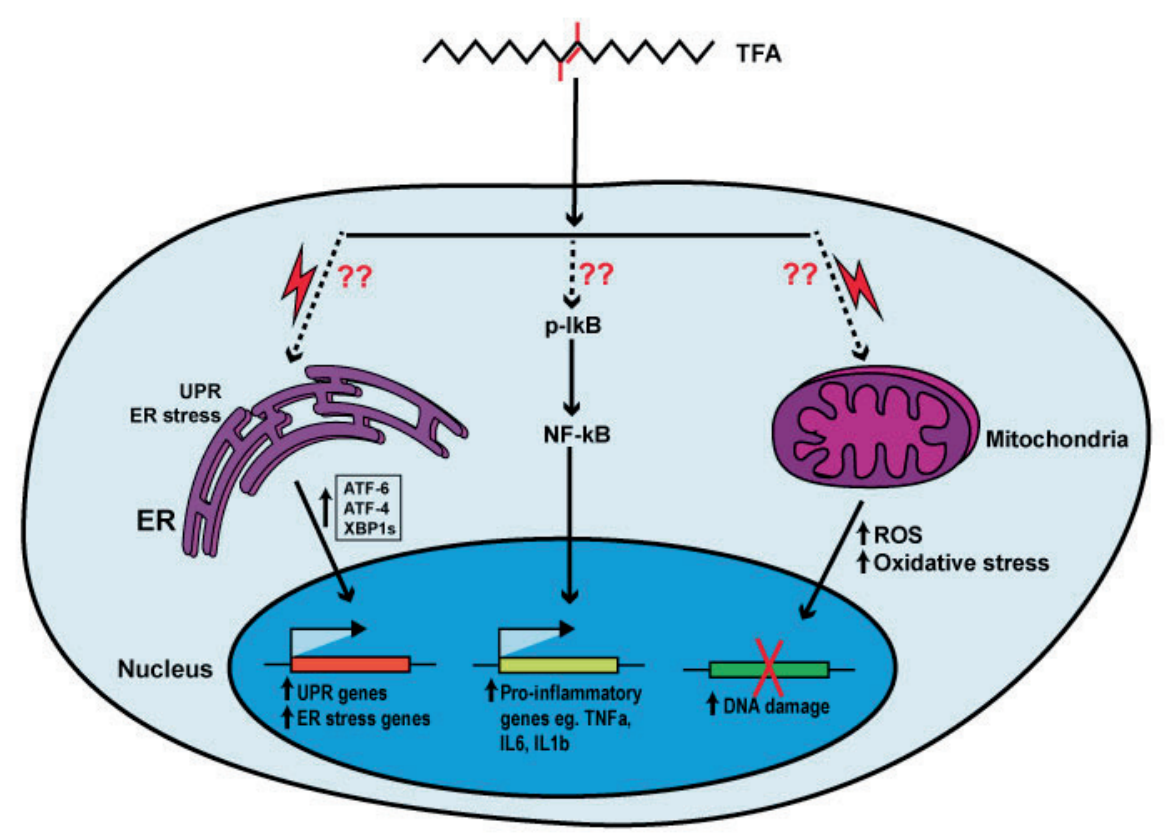

Figure 2: Proposed molecular mechanisms of trans fatty acids.

Abbreviations: TFA, trans fatty acid; UPR, unfolded protein response; ER, endoplasmic reticulum; ATF6, Activating transcription factor 6; ATF4, Activating transcription factor 4; XBP1s, X-box binding protein 1, spliced form; NF- $\mathrm{B}$, nuclear factor kappa-B; p-IкB phosphorylated Ikappa B; TNFa, tumor necrosis factor-alpha; IL6, interleukin 6; IL1b, interleukin 1 beta; ROS, reactive oxygen species 


\section{Endoplasmic reticulum (ER) stress, Reactive oxygen species (ROS) production and Oxidative stress}

At the cellular level, environmental, physiological and pathological insults can generate varying degrees of stress, such as ER and oxidative stress, which negatively affect cell function. The ER is a cellular organelle that plays important functions in calcium homeostasis, lipid metabolism, protein synthesis and post-translational modifications. Stress to the ER impairs ER function and results in activation of the unfolded protein response (UPR). The UPR comprises a signalling cascade that promotes cell survival but can also lead to apoptotic cell death if the stress persists [59]. Meanwhile, ROS are highly reactive chemical species that are generated during various biochemical processes. In low and controlled amounts, ROS serve important signalling functions and initiate certain biological processes, such as cell proliferation and inflammation. However, high levels of ROS due to imbalance between ROS production and antioxidant defence can result in oxidative stress and subsequent damage to lipids, DNA and protein $[60,61]$. Certain fatty acids, especially when present in excessive amounts, are known to be injurious to cells due to their modulatory effects on ER stress, ROS production, and oxidative stress [31]. In the study of the molecular mechanism of trans fatty acids, ER and oxidative stress have been invoked as potential mediators of the harmful effects of trans fatty acids (Figure 2). In coronary artery disease patients, a correlation was found between plasma levels of trans fatty acids and the severity of the disease. In these patients, antioxidant enzymes such as erythrocyte glutathione peroxidase and superoxide dismutase activities were significantly decreased concomitant with increases in oxidative stress and lipid peroxidation [14]. A number of animal studies and cell culture experiments have further examined the role of ER stress and oxidative stress as mechanistic mediators of the effects of trans fatty acids. A number of animal studies and cell culture experiments have further examined the impact of ER stress and oxidative stress. In a mouse model of hyperlipidaemia, a diet containing elaidic acid but not oleic acid increased atherosclerotic lesion size [62]. The mice fed elaidic acid showed significant increases in superoxide production and the expression of NADPH oxidase in the aortic vessel wall. The authors recapitulated the in vivo findings in cultured smooth muscle cells incubated with elaidic acid to further underscore the role of ROS and oxidative stress as mediators of trans fatty acid-induced atherosclerosis [62]. In C57B16/J mice [63] and Wistar rats [64], the presence of industrial trans fatty acids in the diet resulted in hepatic lipotoxicity characterized by an increase in oxidative stress and a decrease in antioxidant activity of catalase, superoxide dismutase, and glutathione peroxidase. 
Events in the endothelial lining of blood vessels are known to be important in the pathogenesis of vascular diseases. In human umbilical vein endothelial cells, both elaidic and linoelaidic acid increased ROS production in a dose-dependent manner through increased activity of caspase 3 prior to apoptosis induction [65]. Furthermore, in human microvascular endothelial cells, elaidic and linoelaidic trans fatty acids increased superoxide production leading to vascular inflammation through enhanced NF-kB signalling [56]. One study explored the possible mechanisms behind the neurotoxic effects of elaidic acid in neuroblastoma cell line. It was found that along with increased ER stress, elaidic acid induced ROS production and decreased activity of antioxidants, which resulted in oxidative damage and apoptosis [66]. By contrast, in RAW264.7 macrophages, elaidic acid showed similar inability to induce ER stress as oleic acid. Whereas palmitic acid potently induced ER stress characterized by enhanced mRNA and protein expression of several stress markers including CHOP and XBP1(s), elaidic acid had only modest to no effects [58]. Taken together, there is evidence that compared to cis-unsaturated fatty acids, trans fatty acids may promote oxidative stress and to a lesser extent ER stress, which may contribute to their deleterious effects.

\section{Autophagy}

Autophagy refers to self-cannibalism. Specifically, autophagy describes an adaptive response to stress during which organelles and other intracellular components are degraded in the lysosome as a way of recouping energy substrates for cell survival and maintenance of cell integrity. Nutrient shortage, sub-lethal damage and adaption to changing environmental conditions are important triggers of autophagy. Autophagy involves a complex signalling cascade comprising essentially of phagophore formation, conjugation of autophagy related proteins (ATGs), microtubule-associated protein 1A/1B-light chain 3 (LC3) processing and association with the phagophore membrane, the capture of targets for degradation, fusion between autophagosomes and lysosomes, and the proteolytic degradation of engulfed targets by lysosomal proteases [67,68]. Few studies have investigated fatty acids in general as triggers of autophagy and even less so for trans fatty acids [69]. One study conducted in primary cardiac myofibroblasts showed that trans-vaccenic and elaidic acid induce apoptosis secondary to autophagy induction [70]. Compared to non-treated control, both trans fatty acids stimulated autophagosme formation, LC3- $\beta$ lipidation, LC3-II formation and ATG5-12 accumulation concomitant with elevated levels of cleaved caspases 9, 3, 7 and enhanced translocation of Bax to the mitochondria [70]. This study highlighted trans fatty acids as potent stressors to myofibroblasts culminating in pre-mature apoptosis. A recent study, however, reported contrasting results in U2OS cells that stably expressed different biosensor markers of 
autophagy, unfolded protein response, and golgi stress. In these cells, the trans fatty acids elaidic and trans-vaccenic acid inhibited autophagy induced by saturated fatty acids [71]. The suggestion was raised that the lipotoxicity of trans fatty acids may reside in their ability to inhibit cytoprotective stress response induced by saturated fatty acids [71]. So far, it is rather difficult to make tangible conclusions from these few and seemingly incoherent studies. Additional work is needed to clarify the exact role of trans fatty acids in autophagy and its implication on cardiometabolic health.

\section{Gut microbiota}

The human gut contains trillions of bacteria that are categorized into over 1,000 species. These microbes are important for shaping the intestinal immune response and for the degradation of complex polysaccharides, leading to the production of metabolites such as short chain fatty acids (SCFAs) [72,73]. Studies of the gut microbial populations have revealed an association between changes in gut microflora and certain human diseases such as obesity, inflammatory bowel disease, diabetes, cardiovascular diseases and cancer [74-77]. It is now known that a harmonious symbiotic relationship between the resident gut microbes is important to maintain good health. A disturbance in harmony and biodiversity due to external factors such as diet can promote the outgrowth of pathogenic or opportunistic subpopulation. In humans and mice, diets rich in certain types of fatty acids have been suggested to alter metabolic health due in part to a reorganisation of certain species of the gut microbiome [78,79]. A recent study specifically investigated the effect of a diet enriched with industrial trans fatty acids from partially hydrogenated soybean oil on the gut microbiota in C57BL/6 mice. Compared to a control diet that contained no trans fatty acids, the trans fatty acid-enriched diet not only resulted in liver steatosis and intestinal inflammation, but also increased relative abundances of 'harmful' Proteobacteria and Desulfovibrionaceae, and decreased abundances of 'beneficial' bacteria that belong to Bacteroidetes, Lachnospiraceae, and Bacteroidales S24-7 [80]. Studies on trans fatty acid regulation on gut microbiota are very few. Accordingly, whether the gut microbiota have a causal role in mediating the effects of trans fatty acids on cardiometabolic disorders is unclear and requires further investigation.

\section{Molecular effects of trans fatty acids on liver}

The liver serves as the hub of lipid metabolism. In the liver, an intricate balance exists between the uptake, storage, synthesis, and oxidation of lipids in order to maintain local and systemic lipid homeostasis. Aberrant hepatic lipid metabolism can lead to dyslipidaemia and non-alcoholic fatty liver disease (NAFLD), both of which are 
important risk factors for cardiovascular disorders [81,82]. A number of studies have shown that the liver bears the brunt of trans fatty acid-mediated pathology. In mice, the intake of diets rich in industrial trans fatty acids has been shown to promote liver damage, characterised by elevated levels of plasma alanine aminotransferase activity and increased plasma levels of acute phase proteins including serum amyloid A and haptoglobin [53,83-85]. Furthermore, histological staining of liver slices have showed profound fat accumulation in mice fed diets rich in industrial trans fatty acids as well as histological indications of NASH and cirrhosis [53,54,86,87]. In a number of animal studies, the steatotic liver phenotype was associated with increased expression of genes involved in lipogenesis, including Fasn, Acaca, and Srebp 1 [54,84,87]. In a recent study in Chapter 3 of this thesis, we found that a diet rich in industrial trans fatty acids markedly increased the ratio of liver to gonadal fat mass, plasma alanine aminotransferase activity, and plasma acute phase proteins in comparison with diets enriched in cis-unsaturated and saturated fatty acids. Additionally, the diet rich in industrial trans fatty acids increased steatosis, hepatic cholesterol levels, and fibrosis markers, suggesting enhanced NAFLD.

In conjunction with these studies in mice, several studies have examined the effect of elaidic acid on lipid metabolism in human and murine hepatoma cells. It was found that elaidic acid stimulated SREBP1c-dependent lipid synthesis in human Huh-7 cells through enhanced expression SREBP target genes [88]. Nielsen and colleagues applied an integrated lipidomics, transcriptomics and proteomics approach in human HepG2 cells to demonstrate that elaidic acid, but not oleic or palmitic acid, potently enhanced expression of key enzymes involved in cholesterol and fatty acid biosynthesis. Relative to oleic acid, elaidic acid upregulated SREBP2 expression along with several other cholesterogenic genes such as HMGCo-A reductase, squalene epoxidase and mevalonate kinase [89]. We recapitulated the findings of Nielsen and colleagues in murine Hepa1-6 hepatocytes and showed an unequivocal role of SREBP2 in mediating the effect of trans fatty acids on lipid/cholesterol metabolism (Chapter 3 of this thesis). Specifically, we found that elaidic acid potently upregulates the expression of genes involved in cholesterol synthesis and induces the expression and activity of SREBP2. Silencing of Srebp2 by siRNA-mediated knockdown abrogates the cholesterogenic effect of elaidic acid. The Srebp 2 activation by elaidic acid is likely due to lowered levels of intracellular free cholesterol since cholesterol is a negative regulator of the SREBP signalling pathway. A previous study reported that elaidic acid is a high affinity substrate for the esterification of cholesterol into cholesterol esters [90], which might explain why elaidic acid reduces levels of intracellular free cholesterol. In support of this hypothesis, elaidic 
acid was shown to increase the expression of the cholesterol esterification enzyme, Sterol O-acyltransferase 1 (Soat1) [89]. In our hands, although elaidic acid significantly lowered levels of free cholesterol, there was no concomitant increase in levels of cholesterol ester. Furthermore, silencing of Soatl did not abrogate the induction of cholesterol synthesis genes by elaidic acid. Accordingly, enhanced cholesterol esterification likely does not explain the increase in SREBP2 activity by elaidic acid. Rather, elaidic acid appears to decrease the sensitivity of SCAP to cholesterol (Chapter 3 of thesis). Overall, the cholesterogenic effect of elaidic acid can at least partly be ascribed to its ability to reduce the levels of intracellular free cholesterol and decrease the sensitivity of SCAP to cholesterol. This anabolic effect of elaidic acid may contribute to the deleterious effect of industrial trans fatty acids on lipid metabolism.

\section{Molecular effect of trans fatty acids on adipose tissue}

White adipose tissue is the principle organ responsible for the storage of excess energy and fat. Compared to the liver, fewer studies have reported the effects of trans fatty acids on adipose tissue. Most human studies that have investigated the effects of trans fatty acids on adipose tissue have focussed on conjugated linoleic acids (CLAs). CLAs are a distinct class of naturally occurring trans fatty acids that require a full review on its own, as done elsewhere $[91,92]$ and therefore will only be discussed briefly here. In the United States, 10E,12Z CLA is marketed commercially as a natural weight loss supplement due to its reported ability to reduce adipose tissue mass $[93,94]$. Notwithstanding their potential health benefits, a number of studies have revealed that intake of CLAs may cause negative side effects such as increasing plasma markers of inflammation and oxidative stress [93], liver damage [95], and inflammation in macrophages [96]. In mice, diets that contain 10E,12Z CLA remarkably reduced fat mass yet at the same time promoted hyperinsulinemia, adipose tissue inflammation, and liver damage [97-100]. In 3T3-L1 adipocytes, 10E,12Z CLA enhanced lipid utilization by stimulating lipolysis and fatty acid oxidation, thereby impairing triglyceride storage [101]. The increased lipid oxidation was associated with increased mitochondrial ROS production and a proinflammatory response, characterised by increased expression of McP- 1 and Il-6 [101].

Apart from CLAs, a diet enriched with elaidic acid reduced fat tissue mass in LDLreceptor knockout mice along with an increase in liver mass and liver steatosis [87]. Consistent with these findings, in Chapter 3 of this thesis, we show that feeding wildtype C57BL6/J mice a high fat diet rich in industrial trans fatty acids reduced adipose tissue mass but increased liver mass compared with isocaloric diets rich in cis- 
unsaturated or saturated fatty acids. The decrease in adipose tissue mass was accompanied by a strong upregulation of genes involved in fatty acid and cholesterol synthesis in both gonadal and inguinal fat tissue depots [102]. The evidence from these studies suggests that diets rich in trans fatty acids cause preferential fat accumulation in the liver at the expense of fat tissues. The fat tissue seems to compensate for this anomaly by stimulating de-novo lipogenesis. What molecular mediators account for preferential fat trafficking to the liver is unknown. These intriguing observations warrant further investigation, as any mechanistic insight gained could be utilised to redirect fat towards specific tissues under different physiological states to avert ectopic fat accumulation and potentially diminish NAFLD.

\section{Molecular effects between industrial and ruminant trans fatty acids}

Public health policies that are meant to restrict consumption of trans fatty acids are rendered less efficient due to the existence of ruminant trans fatty acids. As previously mentioned, trans fatty acids can be of industrial or ruminant origin. The concentration of industrial trans fatty acids in partially hydrogenated vegetable oils can be as high as $60 \%$, with elaidic acid as main trans fatty acid [27,103]. Trans-vaccenic acid is the most abundant ruminant trans fatty acid and represents $50-80 \%$ of total ruminant trans fatty acid intake [26,104]. Previous understanding that all trans fatty acids have similar pathophysiological effects has proven less accurate in light of evidences emerging from studies that discriminated between industrial and ruminant trans fatty acids. In a metaanalysis by Brendsten and colleagues, only intake of industrial but not ruminant trans fatty acids showed a positive association with coronary heart disease [25]. In LDLreceptor knockout mice, elaidic acid enhanced the progression of atherosclerosis by stimulating inflammation and oxidative stress, whereas trans-vaccenic acid decreased aortic atherosclerotic plaque formation [62]. It can be hypothesized that the different position of the trans double bonds in elaidic and trans-vaccenic acid may lead to differences in fatty acid signalling, cellular transport, and incorporation into cell membranes [105].

A number of in vitro studies also underscore the different properties of industrial and ruminant trans fatty acids. In HepG2 cells, elaidic acid increased protein levels of enzymes involved in cholesterol synthesis and transport, whereas trans-vaccenic acid showed no such effect [106]. Similarly, in Hepa1-6 and 3T3-L1 adipocytes, elaidic acid distinctly induced the expression of cholesterol and fatty acid synthesis genes in contrast to trans-vaccenic acid. Also, Hirata and colleagues showed that elaidic and lionelaidic 
acids but not trans-vaccenic acid induce inflammation and superoxide production in human microvascular endothelial cells [56].

In spite of the reported contrasting effects of industrial and ruminant trans fatty acids, other studies report no such differences [107]. In a quantitative review of the existing literature, Brouwer and colleagues concluded that trans fatty acids raise the ratio of plasma LDL to HDL cholesterol independent of their ruminant or industrial origin [108]. In a randomized controlled study in healthy men, ruminant and industrial trans fatty acid diets had similar effects on plasma lipoproteins by significantly increasing levels of plasma LDL-cholesterol and lowering levels of plasma HDL-cholesterol [109]. Studies in cultured macrophages suggest that industrial and ruminant fatty acids behave similarly with respect to cytotoxicity and inflammation. In RAW264.7 macrophages, both elaidic acid and trans-vaccenic acid induced apoptotic cell death and inflammation [57]. In a separate study in RAW264.7 macrophages, palmitate potently induced gene markers of inflammation and ER stress, which could not be reproduced with either industrial and ruminant trans fatty acids [58].

Taken together, evidence from both pre-clinical and clinical studies shows that industrial and ruminant trans fatty acids have differential effects, depending on the biological pathway and clinical outcome measures under investigation. It is conceivable and indeed suggested that the proposed positive effects of ruminant trans fatty acids could be due to a relatively lower intake compared to industrial trans fatty acids [25]. Nonetheless, the underlying mechanisms for such reported differences are still unclear. From a biochemical perspective, the differences in the source and slight change in the position of the trans double bond may not justify the separate classification of industrial and ruminant fatty acids. However, there are numerous examples of bioactive molecules in which a slight change in molecular structure has a profound impact on the biological properties. Indeed, the position of the trans double bond could impact the extent to which fatty acids are taken up, sensed, metabolised, and incorporated into cellular organelles and membranes. Such potential mechanisms are still poorly delineated and warrant further investigation.

\section{Conclusion}

Epidemiological studies have indicated that intake of industrial trans fatty acids is associated with increased risk of cardiovascular disease. Clinical studies in humans have shown that this association is likely explained by an increase in total and LDL cholesterol levels and a decrease in HDL cholesterol levels by industrial trans fatty acids. Preclinical studies in mice and cultured cells have partly clarified the molecular mechanisms 
of action of trans fatty acids. In cultured cells, trans fatty acids stimulate inflammation, ER stress, and oxidative stress, although less potently than saturated fatty acids. Besides impacting on inflammation and stress-related pathways, trans fatty acids have a profound influence on lipid metabolism. In mice, industrial trans fatty acids promote the preferential storage of fat in the liver at the expense of fat tissue. Studies in cultured liver cells indicate that industrial trans fatty acids stimulate the cholesterol synthesis pathway by activating SREBP2-mediated gene regulation.

Overall, the reported distinct effects of industrial and ruminant trans fatty acids in vitro and in vivo prevents the grouping of all trans fatty acids as a single entity. From an evolutionary perspective, certain animals have conserved the ability to endogenously synthesize certain types of trans fatty acids, rendering it unsurprising that such ruminant trans fatty acids may carry certain benefits. Presently, there is still limited understanding of the mechanism of action of trans fatty acids. Accordingly, further mechanistic studies are required to fully clarify both defined and undefined properties of industrial and ruminant trans fatty acids. Gaining deeper understanding of the molecular mechanism of action of trans fatty acids may create new therapeutic windows for the treatment of diseases characterised by disrupted lipid metabolism. 


\section{References}

[1] Ginter, E., Simko, V., 2016. New data on harmful effects of trans-fatty acids. Bratislavské Lekárske Listy 117(5): 251-3.

[2] Filip, S., Vidrih, R., 2008. Trans Fatty Acids and Human Health 16.

[3] Gotoh, N., Kagiono, S., Yoshinaga, K., Mizobe, H., Nagai, T., Yoshida, A., et al., 2018. Study of Trans Fatty Acid Formation in Oil by Heating Using Model Compounds 281(3): 273-81.

[4] Johnston, P. V., Johnson, O.C., Kummerow, F.A., 1957. Occurrence of trans Fatty Acids in Human Tissue. Science 126(3276): 698-9.

[5] Thomas, L.H., Winter, J.A., Scott, R.G., 1983. Concentration of 18:1 and 16:1 transunsaturated fatty acids in the adipose body tissue of decedents dying of ischaemic heart disease compared with controls: Analysis by gas liquid chromatogrpahy. Journal of Epidemiology and Community Health 37(1): 16-21.

[6] Thomas, L.H., Winter, J.A., Scott, R.G., 1983. Concentration of transunsaturated fatty acids in the adipose body tissue of decedents dying of ischaemic heart disease compared with controls. Journal of Epidemiology and Community Health 37(1): $22-$ 4.

[7] Mensink, R.P.\&., Katan, M.B., 1990. Effect of dietary trans fatty acids on highdensity and low-density lipoprotein cholesterol levels in healthy subjects. New England Journal of Medicine 327(2): 82-7.

[8] WILLETT, W., 1993. Intake of trans fatty acids and risk of coronary heart disease among women. The Lancet 341(8845): 581-5.

[9] Thomas, L.H., 1992. Ischaemic heart disease and consumption of hydrogenated marine oils in England and Wales. Journal of Epidemiology and Community Health 46(1): 78-82.

[10] Ganguly, R., Pierce, G.N., 2012. Trans fat involvement in cardiovascular disease. Molecular Nutrition and Food Research 56(7): 1090-6.

[11] Mozaffarian, D., Katan, M.B., Ascherio, A., Stampfer, M.J., Willett, W.C., 2006. Trans Fatty Acids and Cardiovascular Disease. New England Journal of Medicine 354(15): 1601-13.

[12] Ascherio, a., Hennekens, C.H., Buring, J.E., Master, C., Stampfer, M.J., Willett, W.C., 1994. Trans-fatty acids intake and risk of myocardial infarction. Circulation 89: 94-101.

[13] Kummerow, F.A., 2009. The negative effects of hydrogenated trans fats and what to do about them. Atherosclerosis 205(2): 458-65.

[14] Ahmed, S.H., Kharroubi, W., Kaoubaa, N., Zarrouk, A., Batbout, F., Gamra, H., et al., 2018. Correlation of trans fatty acids with the severity of coronary artery disease lesions. Lipids in Health and Disease 17(1): 1-13.

[15] Stender, S., Dyerberg, J., Astrup, A., 2006. Consumer protection through a legislative ban on industrially produced trans fatty acids in foods in Denmark. Scandinavian Journal of Food \& Nutrition 50(July): 155-60. 
[16] Pérez-Farinós, N., Dal Re Saavedra, M.Á., Villar Villalba, C., Robledo de Dios, T., 2016. Trans-fatty acid content of food products in Spain in 2015. Gaceta Sanitaria 30(5): 379-82.

[17] Kaur, G., Cameron-Smith, D., Sinclair, A.J., 2012. Are trans fats a problem in Australia? The Medical Journal of Australia 196(11): 666-7.

[18] Roe, M., Pinchen, H., Church, S., Elahi, S., Walker, M., Farron-Wilson, M., et al., 2013. Trans fatty acids in a range of UK processed foods. Food Chemistry 140(3): 427-31.

[19] Karabulut, I., 2007. Fatty acid composition of frequently consumed foods in Turkey with special emphasis on trans fatty acids. International Journal of Food Sciences and Nutrition 58(8): 619-28.

[20] Monge-Rojas, R., Colón-Ramos, U., Jacoby, E., Alfaro, T., Tavares do Carmo, M.D.G., Villalpando, S., et al., 2017. Progress towards elimination of trans-fatty acids in foods commonly consumed in four Latin American cities. Public Health Nutrition (12): 1-10.

[21] Remig, V., Franklin, B., Margolis, S., Kostas, G., Nece, T., Street, J.C., 2010. Trans Fats in America: A Review of Their Use, Consumption, Health Implications, and Regulation. Journal of the American Dietetic Association 110(4): 585-92.

[22] Zupanič, N., Hribar, M., Pivk Kupirovič, U., Kušar, A., Žmitek, K., Pravst, I., 2018. Limiting trans Fats in Foods: Use of Partially Hydrogenated Vegetable Oils in Prepacked Foods in Slovenia. Nutrients 10(3): 355.

[23] Craig-Schmidt, M.C., 2006. World-wide consumption of trans fatty acids. Atherosclerosis Supplements 7(2): 1-4.

[24] Resnik, D., 2010. Trans Fat Bans and Human Freedom. Am J Bioeth. 10(3): 27-32.

[25] Bendsen, N.T., Christensen, R., Bartels, E.M., Astrup, A., 2011. Consumption of industrial and ruminant trans fatty acids and risk of coronary heart disease: A systematic review and meta-analysis of cohort studies. European Journal of Clinical Nutrition 65(7): 773-83.

[26] Ganguly, R., Pierce, G.N., 2015. The toxicity of dietary trans fats. Food and Chemical Toxicology 78: 170-6.

[27] Stender, S., Astrup, A., Dyerberg, J., 2008. Ruminant and industrially produced trans fatty acids: Health aspects. Food and Nutrition Research 52: 1-8.

[28] Tvrzicka, E., Kremmyda, L.S., Stankova, B., Zak, A., 2011. Fatty acids as biocompounds: Their role in human metabolism, health and disease - a review. part 1: Classification, dietary sources and biological functions. Biomedical Papers 155(2): 117-30.

[29] Koletzko, B., Decsi, T., 1997. Metabolic aspects of trans fatty acids. Clinical Nutrition 16(5): 229-37.

[30] Bassett, C.M.C., McCullough, R.S., Edel, A.L., Maddaford, T.G., Dibrov, E., Blackwood, D.P., et al., 2009. trans-Fatty acids in the diet stimulate atherosclerosis. Metabolism: Clinical and Experimental 58(12): 1802-8.

[31] Estadella, D., Claudia, M., Oller, P., Piano, A. De., 2014. Lipotoxicity : Effects of 
Dietary Saturated and Transfatty Acids. Hindawi 2013: 1-15.

[32] Zapolska, D.D., Bryk, D., Olejarz, W., 2015. Trans Fatty Acids and Atherosclerosiseffects on Inflammation and Endothelial Function. Journal of Nutrition \& Food Sciences 05(06).

[33] Palazón-Bru, A., Carbayo-Herencia, J.A., Simarro-Rueda, M., Artigao-Ródenas, L.M., Divisón-Garrote, J.A., Molina-Escribano, F., et al., 2018. Comparison Between Non-High-Density Lipoprotein Cholesterol and Low-Density Lipoprotein Cholesterol to Estimate Cardiovascular Risk Using a Multivariate Model. The Journal of Cardiovascular Nursing.

[34] Ramasamy, I., 2014. Recent advances in physiological lipoprotein metabolism. Clinical Chemistry and Laboratory Medicine 52(12): 1695-727.

[35] Chan, D.C., Watts, G.F., 2006. Apolipoproteins as markers and managers of coronary risk. QJM - Monthly Journal of the Association of Physicians 99(5): 277-87.

[36] Zock, P.L., Katan, M.B., 1992. Hydrogenation alternatives: effects of trans fatty acids and stearic acid versus linoleic acid on serum lipids and lipoproteins in humans. Journal of Lipid Research 33(3): 399-410.

[37] Roos, N.M. de., Schouten, E.G., Katan, M.B., 2001. Consumption of a solid fat rich in lauric acids results in a more favourable serum lipid profile in healthy men and women than cosumption of a solid fat rich in trans-faty acids. Journal of Nutrition 131(2): 242-5.

[38] Judd, J.T., Baer, D.J., Clevidence, B.A., Kris-Etherton, P., Muesing, R.A., Iwane, M., 2002. Dietary cis and trans monounsaturated and saturated FA and plasma lipids and lipoproteins in men. Lipids 37(2): 123-31.

[39] Mauger, J.F., Lichtenstein, A.H., Ausman, L.M., Jalbert, S.M., Jauhiainen, M., Ehnholm, C., et al., 2003. Effect of different forms of dietary hydrogenated fats on LDL particle size. American Journal of Clinical Nutrition 78(3): 370-5.

[40] Matthan, N.R., Welty, F.K., Barrett, P.H.R., Harausz, C., Dolnikowski, G.G., Parks, J.S., et al., 2004. Dietary hydrogenated fat increases high-density lipoprotein apoAI catabolism and decreases low-density lipoprotein apoB-100 catabolism in hypercholesterolemic women. Arteriosclerosis, Thrombosis, and Vascular Biology 24(6): 1092-7.

[41] Judd, J.T., Baer, D.J., Clevidence, B.A., Muesing, R.A., Chen, S.C., Weststrate, J.A., et al., 1998. Effects of margarine compared with those of butter on blood lipid profiles related to cardiovascular disease risk factors in normolipemic adults fed controlled diets. American Journal of Clinical Nutrition 68(4): 768-77.

[42] Nestel, P., Noakes, M., Belling, B., McArthur, R., Clifton, P., Janus, E., et al., 1992. Plasma lipoprotein lipid and $\mathrm{Lp}$ [a] changes with substitution of elaidic acid for oleic acid in the diet. Journal of Lipid Research 33(7): 1029-36.

[43] Lichtenstein, A.H., Ausman, L.M., Jalbert, S.M., Schaefer, E.J., 1999. Lipoprotein Cholesterol Levels. N Engl J Med.: 1933-40.

[44] Lee, B.C., Lee, J., 2014. Cellular and molecular players in adipose tissue inflammation in the development of obesity-induced insulin resistance. Biochimica et Biophysica Acta - Molecular Basis of Disease 1842(3): 446-62. 
[45] Paoletti, R., 2004. Inflammation in Atherosclerosis and Implications for Therapy. Circulation 109(23): 20-6.

[46] Hansson, G.K., 2005. Inflammation, atherosclerosis, and coronary artery disease. N Engl J Med 352(16): 1685-95.

[47] Baer, D.J., Judd, J.T., Clevidence, B.A., Tracy, R.P., 2004. Dietary fatty acids affect plasma markers of inflammation in healthy men fed controlled diets:a randomized crossover study. The American Journal of Clinical Nutrition 79(July): 969-73.

[48] Han, S.N., Leka, L.S., Lichtenstein, A.H., Ausman, L.M., Schaefer, E.J., Meydani, S.N., 2002. Effect of hydrogenated and saturated, relative to polyunsaturated, fat on immune and inflammatory responses of adults with moderate hypercholesterolemia. Journal of Lipid Research 43(3): 445-52.

[49] Lopez-garcia, E., Schulze, M.B., Meigs, J.B., Manson, J.E., Rifai, N., Stampfer, M.J., et al., 2005. Consumption of Trans Fatty Acids Is Related to Plasma Biomarkers of Inflammation and Endothelial Dysfunction 1. Nutritional Epidemiology 135(3): $562-6$.

[50] Mozaffarian, D., Rimm, E.B., King, I.B., Lawler, R.L., Mcdonald, G.B., Levy, W.C., 2004. trans Fatty acids and systemic inflammation in heart failure. Am J Clin Nutr 80(April): 1521-5.

[51] Afonso, M.S., Lavrador, M.S.F., Koike, M.K., Cintra, D.E., Ferreira, F.D., Nunes, V.S., et al., 2016. Dietary interesterified fat enriched with palmitic acid induces atherosclerosis by impairing macrophage cholesterol efflux and eliciting inflammation. Journal of Nutritional Biochemistry 32: 91-100.

[52] Machado, R.M., Nakandakare, E.R., Quintao, E.C.R., Cazita, P.M., Koike, M.K., Nunes, V.S., et al., 2012. Omega-6 polyunsaturated fatty acids prevent atherosclerosis development in LDLr-KO mice, in spite of displaying a proinflammatory profile similar to trans fatty acids. Atherosclerosis 224(1): 66-74.

[53] Tetri, L.H., Basaranoglu, M., Brunt, E.M., Yerian, L.M., Neuschwander-Tetri, B.A., 2008. Severe NAFLD with hepatic necroinflammatory changes in mice fed trans fats and a high-fructose corn syrup equivalent. AJP: Gastrointestinal and Liver Physiology 295(5): G987-95.

[54] Hu, X., Tanaka, N., Guo, R., Lu, Y., Nakajima, T., Gonzalez, F.J., et al., 2017. PPAR $\alpha$ protects against trans-fatty-acid-containing diet-induced steatohepatitis. Journal of Nutritional Biochemistry 39: 77-855.

[55] Larner, D.P., Morgan, S.A., Gathercole, L.L., Doig, C.L., Guest, P., Weston, C., et al., 2016. Male 11-HSD1 knockout mice fed trans-fats and fructose are not protected from metabolic syndrome or nonalcoholic fatty liver disease. Endocrinology 157(9): 3493-504.

[56] Iwata, N.G., Pham, M., Rizzo, N.O., Cheng, A.M., Maloney, E., Kim, F., 2011. Trans fatty acids induce vascular inflammation and reduce vascular nitric oxide production in endothelial cells. PLoS ONE 6(12): 1-6.

[57] Hirata, Y., Takahashi, M., Kudoh, Y., Kano, K., Kawana, H., Makide, K., et al., 2017. Trans-fatty acids promote proinflammatory signaling and cell death by stimulating the apoptosis signal-regulating kinase 1 (ASK1)-p38 pathway. The Journal of 
Biological Chemistry 1(6).

[58] Oteng, A.-B., Bhattacharya, A., Brodesser, S., Qi, L., Tan, N.S., 2017. Feeding Angpt14- / - mice trans fat promotes foam cell formation in mesenteric lymph nodes without leading to ascites. J. Lipid Res. 58: 1-36.

[59] Han, J., Kaufman, R.J., 2016. The role of ER stress in lipid metabolism and lipotoxicity. Journal of Lipid Research 57(8): 1329-38.

[60] Betteridge JD., 2000. What Is Oxidative Stress? Metabolism - Clinical and Experimental 49(2): 3-8.

[61] Schieber, M., Chandel, N.S., 2014. ROS function in redoz signaling and oxidative stress. Current Biology 24(10): R453-62.

[62] Monguchi, T., Hara, T., Hasokawa, M., Nakajima, H., Mori, K., Toh, R., et al., 2017. Excessive intake of trans fatty acid accelerates atherosclerosis through promoting inflammation and oxidative stress in a mouse model of hyperlipidemia. Journal of Cardiology 70(2): 121-7.

[63] Santos, J.D.B., Mendonça, A.A.S., Sousa, R.C., Silva, T.G.S., Bigonha, S.M., Santos, E.C., et al., 2018. Food-drug interaction: Anabolic steroids aggravate hepatic lipotoxicity and nonalcoholic fatty liver disease induced by trans fatty acids. Food and Chemical Toxicology 116(April): 360-8.

[64] Dhibi, M., Brahmi, F., Mnari, A., Houas, Z., Chargui, I., Bchir, L., et al., 2011. The intake of high fat diet with different trans fatty acid levels differentially induces oxidative stress and non alcoholic fatty liver disease (NAFLD) in rats. Nutrition \& Metabolism 8(1): 65.

[65] Zapolska-Downar D, Kosmider A, N.M., 2005. Trans fatty acids induce apoptosis in human endothelial cells. 1. J Physiol Pharmacol 56(4): 611-25.

[66] Ma, W., Zhao, L., Yuan, L., Yu, H., Wang, H., Gong, X., et al., 2017. Elaidic acid induces cell apoptosis through induction of ROS accumulation and endoplasmic reticulum stress in SH-SY5Y cells. Molecular Medicine Reports: 9337-46.

[67] Glick, D., Barth, S., Macleod, K.F., 2010. Autophagy: cellular and molecular mechanisms. Journal of Pathology The 221(1): 3-12.

[68] Mariño, G., Niso-Santano, M., Baehrecke, E.H., Kroemer, G., 2014. Selfconsumption: The interplay of autophagy and apoptosis. Nature Reviews Molecular Cell Biology 15(2): 81-94.

[69] Wang, J., Nie, D., 2015. Modulation of Autophagy by Free Fatty Acids. Cell Death - Autophagy, Apoptosis and Necrosis.

[70] Ghavami, S., Cunnington, R.H., Yeganeh, B., Davies, J.J.L., Rattan, S.G., Bathe, K., et al., 2012. Autophagy regulates trans fatty acid-mediated apoptosis in primary cardiac myofibroblasts. Biochimica et Biophysica Acta - Molecular Cell Research 1823(12): 2274-86.

[71] Sauvat, A., Chen, G., Müller, K., Tong, M., Aprahamian, F., Durand, S., et al., 2018. Trans-Fats Inhibit Autophagy Induced by Saturated Fatty Acids. EBioMedicine.

[72] Ley, R., Turnbaugh, P., Klein, S., Gordon, J., 2006. Microbial ecology: human gut microbes associated with obesity. Nature 444(7122): 1022-3. 
[73] Qin, J., Li, R., Raes, J., Arumugam, M., Burgdorf, K.S., Manichanh, C., et al., 2010. A human gut microbial gene catalogue established by metagenomic sequencing. Nature 464(7285): 59-65.

[74] Jie, Z., Xia, H., Zhong, S.-L., Feng, Q., Li, S., Liang, S., et al., 2017. The gut microbiome in atherosclerotic cardiovascular disease. Nature Communications 8(1): 845.

[75] Walters, W.A., Xu, Z., Knight, R., 2014. Meta-analyses of human gut microbes associated with obesity and IBD. FEBS Letters 588(22): 4223-33.

[76] Wilson Tang, W.H., Hazen, S.L., 2017. The Gut Microbiome and Its Role in Cardiovascular Diseases. Circulation 135(11): 1008-10.

[77] Janssen, A.W.F., Kersten, S., 2017. Potential mediators linking gut bacteria to metabolic health: a critical view. The Journal of Physiology 595(2): 477-87.

[78] Mullin, G.E., 2010. High-fat diet determines the composition of the murine gut microbiome independently of obesity. Nutrition in Clinical Practice 25(3): 310-1.

[79] Singh, R.K., Chang, H.W., Yan, D., Lee, K.M., Ucmak, D., Wong, K., et al., 2017. Influence of diet on the gut microbiome and implications for human health. Journal of Translational Medicine 15(1): 1-17.

[80] Ge, Y., Liu, W., Tao, H., Zhang, Y., Liu, L., Liu, Z., et al., 2018. Effect of industrial trans-fatty acids-enriched diet on gut microbiota of C57BL/6 mice. European Journal of Nutrition.

[81] Faasse, S., Braun, H., Vos, M., 2018. The role of NAFLD in cardiometabolic disease: an update. F1000Research 7(0): 170.

[82] Abd Alamir, M., Goyfman, M., Chaus, A., Dabbous, F., Tamura, L., Sandfort, V., et al., 2018. The Correlation of Dyslipidemia with the Extent of Coronary Artery Disease in the Multiethnic Study of Atherosclerosis. Journal of Lipids 2018.

[83] Koppe, S.W.P., Elias, M., Moseley, R.H., Green, R.M., 2009. Trans fat feeding results in higher serum alanine aminotransferase and increased insulin resistance compared with a standard murine high-fat diet. Am J Physiol Gastrointest Liver Physiol 297(2).

[84] Obara, N., Fukushima, K., Ueno, Y., Wakui, Y., Kimura, O., Tamai, K., et al., 2010. Possible involvement and the mechanisms of excess trans-fatty acid consumption in severe NAFLD in mice. Journal of Hepatology 53(2): 326-34.

[85] Morinaga, M., Kazuyoshi Kon, ${ }^{*}, \dagger$ Hiroaki Saito, Kumiko Arai, Hiromi Kusama, A.U., Shunhei Yamashina, K.I. and S.W., 2015. Sodium 4-phenylbutyrate prevents murine dietary steatohepatitis caused by trans-fatty acid plus fructose. Journal of Clinical Biochemistry and Nutrition 56(3): 166-70.

[86] Jeyapal, S., Putcha, U.K., Mullapudi, V.S., Ghosh, S., Sakamuri, A., Kona, S.R., et al., 2017. Chronic consumption of fructose in combination with trans fatty acids but not with saturated fatty acids induces nonalcoholic steatohepatitis with fibrosis in rats. European Journal of Nutrition.

[87] Machado, R.M., Stefano, J.T., Oliveira, C.P.M.S., Mello, E.S., Ferreira, F.D., Nunes, V.S., et al., 2010. Intake of trans fatty acids causes nonalcoholic steatohepatitis and reduces adipose tissue fat content. The Journal of Nutrition 140(6): 1127-32. 
[88] Shao, F., Ford, D.A., 2014. Elaidic acid increases hepatic lipogenesis by mediating sterol regulatory element binding protein-1c activity in HuH-7 cells. Lipids 49(5): 403-13.

[89] Vendel Nielsen, L., Krogager, T.P., Young, C., Ferreri, C., Chatgilialoglu, C., Nørregaard Jensen, O., et al., 2013. Effects of Elaidic Acid on Lipid Metabolism in HepG2 Cells, Investigated by an Integrated Approach of Lipidomics, Transcriptomics and Proteomics. PLoS ONE 8(9).

[90] Neuschwander-Tetri, B.A., Ford, D.A., Acharya, S., Gilkey, G., Basaranoglu, M., Tetri, L.H., et al., 2012. Dietary trans-fatty acid induced NASH is normalized following loss of trans-fatty acids from hepatic lipid pools. Lipids 47(10): 941-50.

[91] Lehnen, T.E., da Silva, M.R., Camacho, A., Marcadenti, A., Lehnen, A.M., 2015. A review on effects of conjugated linoleic fatty acid (CLA) upon body composition and energetic metabolism. Journal of the International Society of Sports Nutrition 12(1).

[92] Yang, B., Chen, H., Stanton, C., Ross, R.P., Zhang, H., Chen, Y.Q., et al., 2015. Review of the roles of conjugated linoleic acid in health and disease. Journal of Functional Foods 15: 314-25.

[93] Risérus, U., Basu, S., Jovinge, S., Fredrikson, G.N., Ärnlöv, J., Vessby, B., 2002. Supplementation with conjugated linoleic acid causes isomer-dependent oxidative stress and elevated C-reactive protein: A potential link to fatty acid-induced insulin resistance. Circulation 106(15): 1925-9.

[94] Gaullier, J.M., Halse, J., Høivik, H.O., Syvertsen, C., Nurminiemi, M., Hassfeld, C., et al., 2007. Six months supplementation with conjugated linoleic acid induces regional-specific fat mass decreases in overweight and obese. British Journal of Nutrition 97(3): 550-60.

[95] Ramos, R., Mascarenhas, J., Duarte, P., Vicente, C., Casteleiro, C., 2009. Conjugated linoleic acid-induced toxic hepatitis: First case report. Digestive Diseases and Sciences 54(5): 1141-3.

[96] Belda, B.J., Lee, Y., Vanden Heuvel, J.P., 2010. Conjugated linoleic acids and inflammation: Isomer- and tissue-specific responses. Clinical Lipidology 5(5): 699717.

[97] Poirier, H., Shapiro, J.S., Kim, R.J., Lazar, M.A., 2006. Nutritional supplementation with trans-10, cis-12-conjugated linoleic acid induces inflammation of white adipose tissue. Diabetes 55(6): 1634-41.

[98] Terpstra, a H.M., Beynen, a C., Everts, H., Kocsis, S., Katan, M.B., Zock, P.L., 2002. The decrease in body fat in mice fed conjugated linoleic acid is due to increases in energy expenditure and energy loss in the excreta. The Journal of Nutrition 132(5): 940-5.

[99] Tsuboyama-Kasaoka, N., Takahashi, M., Tanemura, K., Kim, H.J., Tange, T., Okuyama, H., et al., 2000. Conjugated linoleic acid supplementation reduces adipose tissue by apoptosis and develops lipodystrophy in mice. Diabetes 49(9): 1534-42.

[100] Clément, L., Poirier, H., Niot, I., Bocher, V., Guerre-Millo, M., Krief, S., et al., 2002. Dietary trans-10,cis-12 conjugated linoleic acid induces hyperinsulinemia and fatty liver in the mouse. Journal of Lipid Research 43(9): 1400-9. 
[101] den Hartigh, L.J., Han, C.Y., Wang, S., Omer, M., Chait, A., 2013. 10E,12Zconjugated linoleic acid impairs adipocyte triglyceride storage by enhancing fatty acid oxidation, lipolysis, and mitochondrial reactive oxygen species. Journal of Lipid Research 54(11): 2964-78.

[102] Collison, K.S., Maqbool, Z., Saleh, S.M., Inglis, A., Makhoul, N.J., Bakheet, R., et al., 2009. Effect of dietary monosodium glutamate on trans fat-induced nonalcoholic fatty liver disease. Journal of Lipid Research 50(8): 1521-37.

[103] Kuhnt, K., Baehr, M., Rohrer, C., Jahreis, G., 2011. Trans fatty acid isomers and the trans-9/trans-11 index in fat containing foods. European Journal of Lipid Science and Technology 113(10): 1281-92.

[104] Field, C.J., Blewett, H.H., Proctor, S., Vine, D., 2009. Human health benefits of vaccenic acid. Applied Physiology, Nutrition, and Metabolism 34(5): 979-91.

[105] Bassett, C.M.C., Edel, A.L., Patenaude, A.F., McCullough, R.S., Blackwood, D.P., Chouinard, P.Y., et al., 2010. Dietary vaccenic acid has antiatherogenic effects in LDLr-/- mice. The Journal of Nutrition 140(1): 18-24.

[106] Krogager, T.P., Nielsen, L.V., Kahveci, D., Dyrlund, T.F., Scavenius, C., Sanggaard, K.W., et al., 2015. Hepatocytes respond differently to major dietary trans fatty acid isomers, elaidic acid and trans-vaccenic acid. Proteome Science 13: 1-14.

[107] Gebauer, S.K., Destaillats, F., Dionisi, F., Krauss, R.M., Baer, D.J., 2015. Vaccenic acid and trans fatty acid isomers from partially hydrogenated oil both adversely affect LDL cholesterol: A double-blind, randomized controlled trial. American Journal of Clinical Nutrition 102(6): 1339-46.

[108] Brouwer, I.A., Wanders, A.J., Katan, M.B., 2010. Effect of animal and industrial Trans fatty acids on HDL and LDL cholesterol levels in humans - A quantitative review. PLoS ONE 5(3): 1-10.

[109] Motard-Bélanger, A., Charest, A., Grenier, G., Paquin, P., Chouinard, Y., Lemieux, S., et al., 2008. Study of the effect of trans fatty acids from ruminants on blood lipids and other risk factors for cardiovascular disease. American Journal of Clinical Nutrition 87(3): 593-9. 
Chapter 3

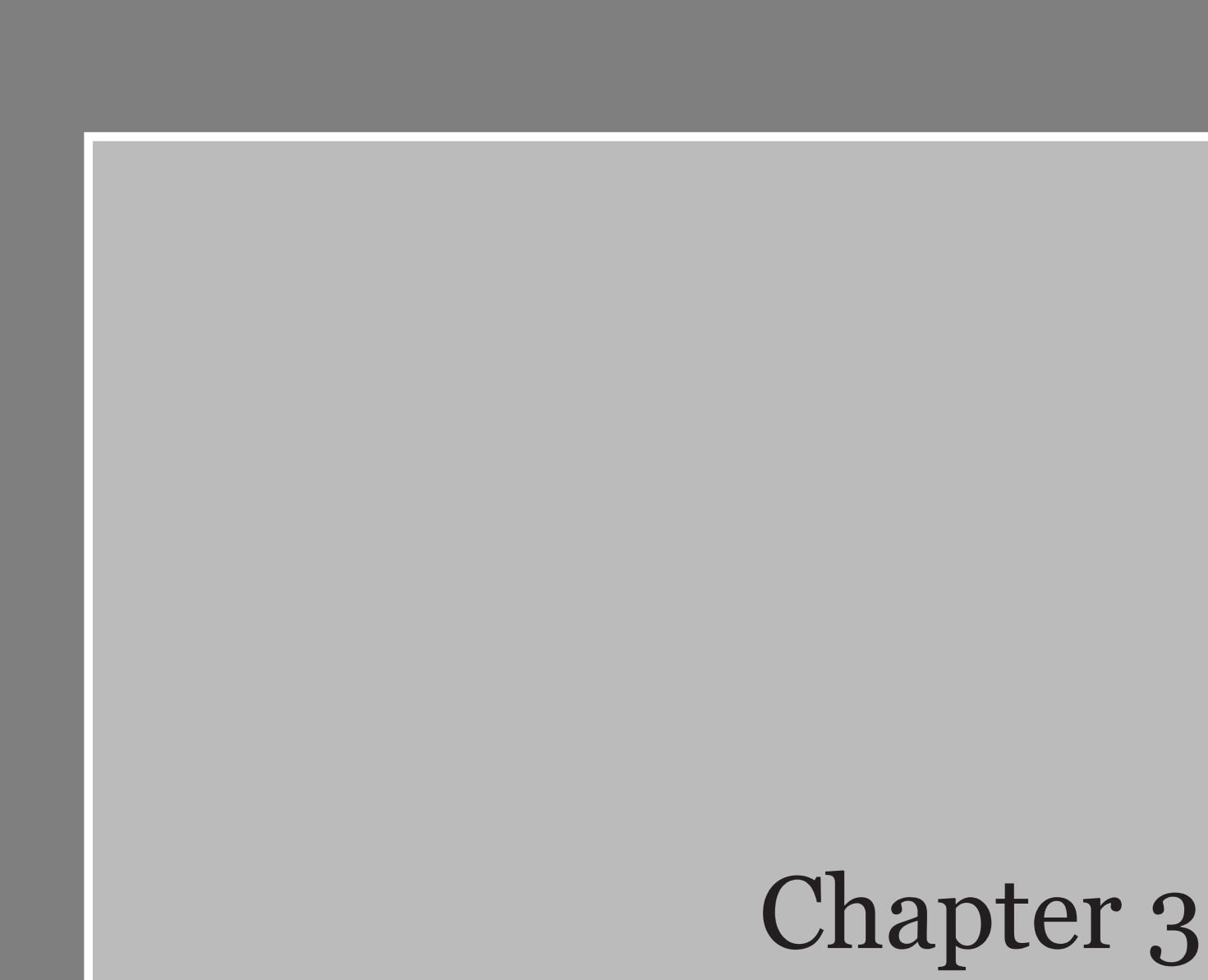




\section{Industrial trans fatty acids}

promote non-alcoholic fatty liver disease and stimulate SREBP2mediated cholesterogenesis

Antwi-Boasiako Oteng, Anke Loregger, Michel van Weeghel, Noam Zelcer, Sander Kersten 


\begin{abstract}
Objective: Consumption of industrial trans fatty acids unfavourably alters plasma cholesterol levels and has been linked to non-alcoholic fatty liver disease (NAFLD). However, the molecular mechanisms underlying these deleterious effects of trans fatty acids are unclear. Here, we aim to investigate the molecular mechanisms of industrial trans fatty acids.
\end{abstract}

Methods: To this end, in vitro experiments were conducted in Hepa1-6 and 3T3-L1 cells incubated with elaidate, oleate, and palmitate as model for trans-unsaturated, cisunsaturated and saturated fatty acids, respectively. Animal experiments were performed in male $\mathrm{C} 57 \mathrm{Bl} / 6$ mice that were fed diets rich in trans-unsaturated, cis-unsaturated or saturated fatty acids for 7 weeks, after which plasma, liver, and adipose tissues were collected and analysed.

Results: Transcriptomics analysis of 3T3-L1 adipocytes and Hepa1-6 cells indicated that elaidate but not oleate or palmitate potently induced expression of genes involved in cholesterol biosynthesis. Induction of cholesterogenesis by elaidate in Hepa1-6 cells was mediated by an increase in SREBP2 and dependent on SCAP, yet independent of LXR and UBXD8. Elaidate decreased intracellular free cholesterol levels and repressed the anti-cholesterogenic effect of exogenous cholesterol. Despite activating the SREPB2 pathway, elaidate decreased abundance of the LDL receptor in an SREBP2-independent and SCAP/SREBP1-dependent manner. In mice, the trans-unsaturated diet markedly increased the ratio of liver to gonadal fat mass, plasma alanine aminotransferase activity, and plasma acute phase proteins relative to the cis-unsaturated and saturated diets. Additionally, the trans-unsaturated diet increased steatosis, hepatic cholesterol levels, and fibrosis markers, suggesting enhanced NAFLD.

Conclusion: Elaidate induces cholesterogenesis via activation of the SCAP-SREBP axis, likely by lowering intracellular free cholesterol and attenuating cholesteroldependent repression of SCAP translocation. This pathway potentially underlies the increase in liver cholesterol and NAFLD by industrial trans fatty acids. 


\section{Introduction}

Trans fatty acids are unsaturated fatty acids with at least one double bond in the trans configuration. Compared to the more common cis-unsaturated fatty acids, transunsaturated fatty acids are less flexible and maintain a straight chain carbon backbone, similar to saturated fatty acids $[1,2]$. Most of the trans fatty acids consumed by humans are industrially produced by partial hydrogenation of vegetable oils and are present in deep fried foods, pastries, cookies, margarine, and popcorns. A small proportion of trans fatty acids are synthesized naturally in the gut of ruminants by bacterial biohydrogenation and are present in meat and dairy products of cattle, goat and sheep [35]. Partially hydrogenated vegetable oils and trans fatty acids are used by the food industry because they confer a combination of beneficial properties to food products, including a long shelf life and a pleasant mouthfeel. In response to growing evidence linking intake of trans fatty acids to coronary heart disease, most food manufacturers and retailers have largely removed trans fatty acids from their products. The 2018 Dutch Nutrition Survey indicated that nowadays, trans fatty acids only provide approximately $0.3 \%$ of the daily energy requirement, as opposed to $5-10 \%$ several decades ago.

In a landmark study in 1990, Mensink and Katan demonstrated the plasma cholesterolraising effect of trans fatty acids in human volunteers [6]. Specifically, they found that trans fatty acids raise plasma LDL cholesterol and reduce plasma HDL cholesterol levels. Following this important finding, multiple epidemiological studies found strong positive associations between consumption of industrial trans fatty acids and the onset and progression of coronary heart disease [7-10]. A recent meta-analysis convincingly established that intake of trans fatty acids is positively associated with all-cause mortality, total coronary heart disease, and coronary heart disease mortality [11].

In addition to coronary heart disease, trans fatty acids have also been connected with other diseases, including non-alcoholic fatty liver disease [12-14]. Indeed, several studies have shown that feeding mice a diet rich in industrial trans fatty acids enhances liver steatosis and features of NASH [15-19]. The increase in liver triglycerides was associated with elevated expression of lipogenic genes, such as Fasn, Acaca, and Srebp1. Remarkably, little is known about the mechanism(s) that underlie the increase in liver triglycerides and lipogenic gene expression in mice fed diets rich in trans fatty acids.

Because the health concerns connected to the consumption of industrial trans fatty acids have been largely allayed by their removal from foods, unfortunately there has been little effort into trying to understand the molecular mechanisms underlying the detrimental effects of trans fatty acids. In a recent study, we found that the industrial trans fatty acid 
elaidate and the saturated fatty acid palmitate had very distinct effects on markers of inflammation and the unfolded protein response in murine RAW264.7 macrophages. In addition, it was observed that elaidate upregulated the expression of putative target genes of sterol regulatory element binding proteins (SREBPs) [20], an observation that was further confirmed in studies in human hepatoma HepG2 and Huh7 cells [21,22].

SREBPs, consisting of SREBP1 and SREBP2, are basic helix-loop-helix-leucine zipper (bHLH-Zip) transcription factors involved in the regulation of cellular lipid metabolism [23-25]. SREBP1 preferentially induces genes involved in fatty acid synthesis, while SREBP2 is more specific for genes involved in cholesterol biosynthesis [26,27]. SREBPs are synthesized as inactive precursors and held in a tripartite complex with SREBP cleavage-activating protein (SCAP) and INSIGs in the endoplasmic reticulum (ER) membrane. In response to low cholesterol levels, INSIG dissociates and the SCAPSREBPs complex is translocated to the Golgi apparatus for proteolytic activation. The resulting active $\mathrm{N}$-terminal SREBP enters the nucleus, where it binds to sterol regulatory elements in the promoter regions of target genes [27-31]. Although both cholesterol and fatty acids are products of SREBP signalling, regulation of SREBP by fatty acids is relatively poorly delineated compared to regulation by cholesterol. Evidence has been presented that poly-unsaturated fatty acids decrease SREBP1 mRNA levels [32-34], stimulate SREBP1 decay [35], inhibit proteolytic processing of SREBP1 via binding to the auxiliary protein UBXD8 [36,37], and antagonize the activity of the liver X receptor, which is a potent inducer of SREBP1 gene transcription [38,39]. How elaidate causes activation of the SREBP pathway remains unclear.

Here, we set out to better understand the mechanism of action of industrialized trans fatty acids. To that end, we pursued two independent experimental strategies. On the one hand, we conducted in vitro experiments in hepatocyte and adipocyte cell lines treated with individual fatty acids, and on the other hand, we fed mice diets enriched in transunsaturated fatty acids, cis-unsaturated fatty acids, and saturated fatty acids, and analyzed the hepatic phenotype. 


\section{Materials and Methods}

\section{Animal treatment}

Animal studies were performed using 4 to 6-month-old pure-bred male mice on a $\mathrm{C} 57 \mathrm{B1} / 6$ background. For the long-term experiment, the mice were randomly assigned to three groups ( $\mathrm{n}=8$ mice per group). For 7 weeks, each group was fed a high-fat diet rich in trans-unsaturated (Trans), cis-unsaturated (Cis) or saturated (Saturated) fatty acids (modified TestDiet 58V8; TestDiet LTD, London). Detailed composition of test diets is provided in Supplemental Table 1. Fat sources were from partially hydrogenated soy oil for the Trans diet, canola and palm olein oil for the Cis diet, and cocoa butter for the Saturated diet. Total fat content in all three diets was $45 \%$ energy percent. Bodyweight and food intake were measured weekly. Following the diet intervention, mice were anesthetized with isoflurane, blood collected via orbital puncture and euthanized by cervical dislocation. Tissues for RNA and protein analysis were immediately frozen in liquid nitrogen and stored at $-80^{\circ} \mathrm{C}$. Tissues for histological analysis were fixed in $4 \%$ paraformaldehyde and later embedded in paraffin. For the short-term experiment, the mice were randomly assigned to the three diet groups $(n=10$ mice per group). The mice were fasted from 12.00 to $18.00 \mathrm{hrs}$, after which they were given access to one of the three diets. The mice were euthanized the next day between 9.00 and $11.00 \mathrm{hrs}$ as described above. The animal studies were approved by the Local Animal Ethics Committee at Wageningen University (2014096.d and AVD104002015236: 2016.W-0093.006).

\section{FA profiling of test diets by gas chromatography-flame ionization detection}

The Folch technique was used to extract the fat component in the test diets as previously described [40]. In summary, a 2:1 mixture of chloroform to ethanol was used to isolate fats from diets. Water was then added to obtain a biphasic system, with the fat present in the lower chloroform phase. The extracted fats were measured gravimetrically after purification followed by saponification with methanolic $\mathrm{NaOH}$ and methylation with boron trifluoride to obtain fatty acid methyl esters. The methyl esters were then fractionated by gas chromatography, detected by flame ionization, and calculated as a fraction of the total amount.

\section{Measurement of plasma parameters}

Blood samples collected into EDTA-coated tubes were centrifuged at 10,000 g for 15 min at $4^{\circ} \mathrm{C}$ to obtain plasma. Plasma levels of non-esterified fatty acids (NEFA) and 
glycerol were measured using kits from HUMAN Diagnostics (Wiesbaden, Germany) according to manufacturer's protocol. Plasma cholesterol and glucose were also quantified using kits from Diasys Diagnostics Systems (Holzheim, Germany) according to manufacturer's protocol. Plasma serum amyloid A (SAA) (Tridelta Development LTD, Ireland) and haptoglobin (Abcam, Cambridge) were measured using ELISA development kits with slight modifications to manufacturer's protocol. Plasma alanine aminotransferase (ALT) activity (Abcam, Cambridge, UK) and insulin (Crystal Chem, Downers Grove, IL) were measured also using dedicated kits.

\section{Liver H \& E staining}

Histology of mice liver samples was performed by Haematoxylin and eosin (H\&E) staining. In summary, paraformaldehyde-fixed liver tissues were processed using ethanol and xylene before being embedded into paraffin blocks. Thin liver sections at $5 \mu \mathrm{m}$ thickness were made using a microtome onto superfrost glass slides and incubated at $37^{\circ} \mathrm{C}$ overnight. The liver slices were then stained for $10 \mathrm{~min}$ in Mayer haematoxylin solution and for $10 \mathrm{sec}$ in eosin Y solution. The slides were then observed under a light microscope and representative images taken.

\section{Liver collagen staining}

Collagen staining of the liver was performed using the Fast Green FCF/Sirius Red staining. In brief, frozen livers were sectioned at $5 \mu \mathrm{m}$, mounted on glass slides and left to air dry for $30 \mathrm{~min}$ at room temperature. The slides were then fixed in $4 \%$ paraformaldehyde for $30 \mathrm{~min}$, washed thrice (1 min each) in absolute ethanol and rinsed briefly with demi water. The tissues were then stained in collagen dye $(0.1 \%$ fast green FCF, $0.1 \%$ direct Red 80 in saturated picric acid solution, Sigma) in a $37{ }^{\circ} \mathrm{C}$ incubator for $90 \mathrm{~min}$. The tissues slices were then rinsed with demi water for 3 to 4 times until the water was clear, dehydrated with absolute ethanol thrice (30 sec each) and in xylene twice (5 min each). The slides were air dried and mounted with Depax mounting medium. Pictures were taken by light microscope.

\section{Liver triglyceride measurement}

To measure liver triglycerides, $2 \%$ liver homogenates were made in a buffer composed of $10 \mathrm{mM}$ Tris, $2 \mathrm{mM}$ EDTA and $25 \mathrm{mM}$ sucrose at $\mathrm{pH} 7.5$ by homogenising in a Tissue Lyser II (Qiagen, Hilden, Germany). Liver triglyceride content was then quantified using Triglyceride liquicolor ${ }^{\text {mono }}$ from HUMAN Diagnostics (Wiesbaden, Germany) according to manufacturer's instructions. 


\section{Liver cholesterol measurement}

Total cholesterol in liver was quantified by gas chromatography-mass spectroscopy (GCMS) as previously described [41]. In brief, a procedure involving extraction, centrifugation and purification steps were used to isolate cholesterol from $200 \mathrm{mg}$ liver samples using $\mathrm{KOH}$, water and hexane as solvents. The extracts along with calibration standards were dissolved in dimethyl formamide (DMF), which also served as mobile phase prior to injection into the GC. The chromatogram that were generated were analysed as total cholesterol content in the mice liver samples.

\section{RNA isolation and quantitative real-time PCR}

Total RNA was isolated from mice tissues, Hepa1-6, Huh7 or 3T3-L1 cells using TRIzol reagent (Invitrogen, Bleiswijk, The Netherlands) and in the case of the mice tissues, homogenised in TRIzol using a Tissuelyser II (Qiagen, Venlo, The Netherlands). Following RNA isolation, 1000 ng of RNA was used to synthesize cDNA by reverse transcription using First strand cDNA synthesis kit (Thermo Fisher-Scientific, Schaumburg, IL) in accordance with manufacturer's protocol. Changes in gene expression were then determined using a quantitative real-time PCR detection system (Bio-Rad) by using SensiMix (Bioline, GC Biotech, Alphen aan den Rijn, The Netherlands). Gene expression values were normalized to the $36 \mathrm{~b} 4$ housekeeping gene. Primer sequences for measured genes are listed in Supplemental Table 2.

\section{Cell culture, 3T3-L1 differentiation and siRNA knockdown}

Cell culture: Hepa1-6 (\# CRL-1830) and 3T3-L1 pre-adipocytes (\# CL-173) were obtained from ATCC (Manassas, VA, USA). Huh-7 cells was a gift from Nicole de Wit (Wageningen University). The cells were cultured in Dulbecco's modified Eagle medium (DMEM) media supplemented with $10 \%$ fetal bovine serum, $100 \mathrm{U} / \mathrm{mL}$ penicillin and $1000 \mu \mathrm{g} / \mathrm{mL}$ streptomycin (Lonza, Verviers, Belgium) in a humidified chamber at $37^{\circ} \mathrm{C}$ with $5 \% \mathrm{CO}_{2}$.

3T3-L1 differentiation: During differentiation, the pre-adipocytes were cultured in 6well plates. Two-days post-confluence, the cell culture media was changed to induction media ( $0.5 \mathrm{mM}$ IBMX, $5 \mu \mathrm{g} / \mathrm{mL}$ insulin, $1 \mu \mathrm{M}$ dexamethasone) for 2 days. Induction media was then replaced with insulin media ( $5 \mu \mathrm{g} / \mathrm{mL}$ insulin) for 3 days, after which the fully differentiated cells were treated with fatty acids.

3T3-L1 co-differentiation with fatty acids: In these experiments, 3T3-L1 were differentiated as described above. During differentiation, the cells were co-treated with $500 \mu \mathrm{M}$ fatty acids. 
siRNA-mediated silencing: Silencing of Srebp1, Srebp2, Scap, Ubxd8 and Soat1 was performed using Dharmacon ON-TARGETplus SMARTpool siRNAs diluted in 1x siRNA buffer. Hepa1-6 cells were plated at $5 \times 10^{4}$ cells per well into 24-well plates and transfected overnight with $50 \mathrm{nM}$ siRNA using $2 \mu \mathrm{L}$ per well Lipofectamine RNAiMAX transfection reagent (Life Technologies, Bleiswijk, The Netherlands). Transfected cells were subsequently treated with $500 \mu \mathrm{M}$ fatty acids for $24 \mathrm{hrs}$.

PLAP-reporter assay: The PLAP assay was performed to measure the processing of SREBP2 in the presence of fatty acids or controls. HEK293 cells were plated at $2 \times 10^{5}$ cells $/ \mathrm{mL}$ into 12-well plates and incubated overnight. The cells were then co-transfected overnight with 150 ng each of PLAP-conjugated SREBP2 plasmids pCMVpCMVSREBP2-PLAP and pcDNA4-hSCAP-MycHis in PEI transfection medium. The transfected cells were washed with PBS and treated for $24 \mathrm{hrs}$ with $500 \mu \mathrm{M}$ of fatty acids: oleate, palmitate, elaidate or vehicle control. Treatments with $50 \mu \mathrm{g} / \mathrm{mL}$ of $\beta$-MCD cholesterol (Sigma-Aldrich) and a combination of simvastatin (Calbiochem) and mevalonic acid (Calbiochem) at $5 \mu \mathrm{g} / \mathrm{mL}$ each served as negative and positive controls respectively. The PLAP assay was then performed by incubating cultured media with PNPP substrate and colorimetrically measuring the absorbance at $405 \mathrm{~nm}$ after $60 \mathrm{~min}$ as previously described [42].

Sub-cellular localisation in CHO SCAP-GFP cells: CHO SCAP-GFP cells were a kind gift of Prof. Espenshade and were described previously [43]. CHO SCAP-GFP cells were cultured at $37^{\circ} \mathrm{C}$ in an atmosphere of $8 \%-9 \% \mathrm{CO}_{2}$, a $1: 1$ mixture of Ham's F-12 medium and DMEM supplemented with $5 \%$ (v/v) FCS. Briefly, cells were generated by transfection of SCAP-deficient SRD-13A cells with pGFP-SCAP, followed by selection in a 1:1 mixture of Ham's F-12 medium and DMEM supplemented with 5\% newborn calf lipoprotein-deficient serum. pGFP-SCAP rescued the cholesterol auxotrophy of SRD-13A cells. CHO SCAP-GFP cells were incubated for 24 hours with $500 \mu \mathrm{M}$ elaidate or control. The cells were analysed by microscopy for subcellular localisation of SCAP.

\section{Fatty acid preparation and treatment}

All fatty acid stocks were initially reconstituted in absolute ethanol. Sub-stocks of fatty acids at $25 \mathrm{mM}$ were prepared in filter-sterilised $\mathrm{KOH}$ at $70 \mathrm{mM}$. Complete DMEM supplemented with fatty acid-free BSA (Sigma-Aldrich, St. Louis, MO) were filter sterilised, and incubated for 30 min with fatty acids to complex BSA to fatty acids. Ratio of BSA to fatty acids were approximately $1: 2$. Vehicle control treatments only contained equivalent mixture of ethanol and KOH. During treatment, Hepa1-6, Huh7 or 3T3-L1 
cells were seeded at $2 \times 10^{5}$ cells $/ \mathrm{mL}$ and incubated overnight, followed by treatment with $500 \mu \mathrm{M}$ concentration of fatty acids for $24 \mathrm{hrs}$. Treatment with any other fatty acids were performed similarly. Differentiated 3T3-L1 cells were incubated with $1 \mathrm{mM}$ final concentrations of fatty acids.

\section{Elaidate treatment in presence of LXR agonist or exogenous cholesterol}

To investigate the role of LXR, elaidate-treated Hepa1-6 cells were co-incubated for 24 hrs with $1 \mu \mathrm{M}$ of GW3965 (Sigma), an LXR agonist. To investigate the effect of elaidate in the presence of elevated cholesterol, Hepa1-6 cells were co-incubated with elaidate in the presence of $500 \mu \mathrm{M}$ of water-soluble cholesterol (Sigma).

\section{Intracellular cholesterol and cholesterol ester quantification by HPLC}

For intracellular cholesterol and cholesterol ester measurement, $4 \times 10^{5}$ cells per well were plated into 6-well plates. After overnight incubation cells were treated with 500 $\mu \mathrm{M}$ of elaidate or control for 24 hours. The cells were then washed three times with icecold PBS and scraped into cold methanol. Cholesterol and cholesterol esters were extracted using a single-phase extraction. A define amount of internal standards consisted of $0.5 \mathrm{nmol}$ of cholesterol ester (CE(14:0)) and $1 \mathrm{nmol}$ cholesterol-D7. The mixture was sonicated in a sonication bath for 15 minutes and centrifuged for 5 minutes at $16,000 \mathrm{~g}$ at $4^{\circ} \mathrm{C}$. The supernatant was transferred to a glass vial and evaporated. The Residue was dissolved in $150 \mu \mathrm{l}$ chloroform/methanol $(1: 1 \mathrm{v} / \mathrm{v})$. The analysis of cholesterol and cholesterol esters was performed using an HPLC-MS system consisting of an Ultimate 3000 binary HPLC pump, a vacuum degasser, a column temperature controller, an auto sampler and a Q-exactive plus mass spectrometer (Thermo Scientific, Waltham, MA, USA). For the cholesterol esters $5 \mu \mathrm{l}$ of the lipid extract was injected onto a "normal phase column" LiChrospher 2x250-mm silica-60 column, $5 \mu \mathrm{m}$ particle diameter (Merck, Darmstadt, Germany) as previously described [44]. For the quantification of cholesterol $5 \mu \mathrm{l}$ of the same lipid extracted was inject onto a "reverse phase column" Acquity UPLC HSS T3, $1.8 \mu \mathrm{m}$ particle diameter (Waters, Milford Massachusetts, USA) and measured as previously described (https://doi.org/10.1016/j.chroma.2014.08.088).

\section{Oil red $O$ staining of lipid droplets}

Oil red-O staining was performed in 3T3-L1 pre-adipocytes co-differentiated with fatty acids. A stock of oil red O (Sigma) was prepared by dissolving $0.5 \mathrm{~g}$ in $500 \mathrm{ml}$ of isopropanol. Working concentrations were made by dissolving stock concentrations with water (3 stock : 2 water) and filtered. Treated cells were washed twice with PBS, 
followed by fixation with $4 \%$ formalin for $30 \mathrm{~min}$. The cells were then washed twice with PBS and incubated with oil red-O dye for $20 \mathrm{~min}$. The stained cells were washed 3 times with $\mathrm{ddH}_{2} \mathrm{O}$ after which cells were visualised under a light microscope and pictures taken.

\section{Western immunoblotting}

Protein expression was determined by western immunoblotting following siRNA knockdown of specific genes and elaidate treatment in Hepa1-6 cells using standard protocols. In summary, proteins were isolated with RIPA lysis buffer containing protease inhibitors and quantified by Pierce BCA protein assay (ThermoScientific, USA) according to manufacturer's protocol. Proteins were prepared and $20 \mu \mathrm{g}$ were loaded onto SDS-PAGE gels, transferred onto PVDF membranes and probed for specific proteins. Primary antibodies for SREBP1 (Millipore, \#MABS1987), SREBP2 (Millipore, \#MABS1988), ACSS2 (Cell Signalling; \#3658S), HMGCR (ATCC CRL1811, IgG-A9, undiluted hybridoma supernatant), LDLR (Biovision, \#3839-100) and Actin (Merck, MAB1501) were diluted in 5\% milk in TBS-T. Secondary antibodies antirabbit IgG-HRP were diluted at 1:5000 in 5\% milk.

\section{Microarray analysis}

Microarray analysis was performed on liver samples from the mice fed the 3 different diets as well as on Hepa1-6 hepatoma and differentiated 3T3-L1 adipocyte cells incubated with different fatty acids. RNA was purified with RNeasy Minikit columns (Qiagen) and analysed for quality with RNA 6000 Nano chips on the Agilent 2100 bioanalyzer (Agilent Technologies, Amsterdam, The Netherlands). One microgram of RNA was used for cDNA synthesis using the First Strand cDNA synthesis kit (Thermo Scientific). Purified RNA (100 ng) was labeled with the Ambion WT expression kit (Invitrogen) and hybridized to an Affymetrix Mouse Gene 1.1 ST array plate (mouse liver, Hepa1-6 cells) or 2.1 ST array plate (3T3-L1 adipocytes) (Affymetrix, Santa Clara, CA). Hybridization, washing, and scanning were carried out on an Affymetrix GeneTitan platform. Scans of the Affymetrix arrays were processed using packages from the Bioconductor project. Arrays were normalized using the robust multi-array average method $[45,46]$. Probe sets were defined by assigning probes to unique gene identifiers, e.g., Entrez ID [47]. For the Hepa1-6 and 3T3-L1 cells, the total gene set (24,973 probe sets) was filtered to only include genes with mean signal $>20$, yielding 10,379 and 11,504 genes, respectively. Microarray data were submitted to the Gene Expression Omnibus (accession number pending). 


\section{Statistical analysis}

Results are presented as mean \pm SEM for animal experiments and mean \pm SD for in vitro experiments. Statistical analyses were performed using Student's paired T-tests or by one-way ANOVA followed by a Turkey's post-hoc multiple comparison test (GraphPad Software, Inc., La Jolla, USA). $\mathrm{P}<0.05$ was considered statistically significant. 


\section{Results}

Elaidate induces a cholesterogenic gene profile in Hepa1-6 and differentiated 3T3-

\section{L1 adipocytes}

To better characterize the molecular pathways activated by industrial trans fatty acids in liver and fat cells, we determined the effects of elaidate on whole genome gene expression in mouse hepatoma Hepa1-6 cells and mouse 3T3-L1 adipocytes. For comparison, cells were also treated with oleate and palmitate as representative cisunsaturated, and saturated fatty acid, respectively. Using a fold-change cut-off of $>1.5$, thirty-one genes were commonly induced by elaidate in Hepa1-6 cells and 3T3-L1 adipocytes (Fig 1A). Pathway analysis of the 31 genes by Enrichr showed strong overrepresentation of pathways related to cholesterol synthesis (Fig 1B). Specifically, of the 31 commonly induced genes, 11 genes are involved in the mevalonate and cholesterol synthesis pathway, including Mvd, Fdft1, Insig1, Dhcr7, Lss (Fig 1C). In contrast to elaidate, oleate downregulated genes involved in cholesterol synthesis, which was most obvious in the Hepa1-6 cells, while palmitate showed an intermediate effect (Fig 1C). QPCR confirmed the induction of cholesterogenic genes in 3T3-L1 adipocytes (Fig 1D) and Hepa1-6 cells (Fig 1E), which was further verified in undifferentiated and differentiating 3T3-L1 cells (Supplemental Fig 1). Since the gene expression changes were most robust in the Hepa1-6 cells, we used Hepa1-6 cells for further investigation into the cellular mode of action of elaidate.

\section{Cholesterogenic effect of elaidate is dependent on SREBP and SCAP}

The expression of cholesterogenic genes is under control of the transcription factors SREBP2 and to a lesser extent SREBP1. Accordingly, we examined the effect of elaidate on the expression of Srebp1 and Srebp2, as well as on additional target genes. Compared to control, elaidate significantly upregulated the expression of Srebp2 and target genes involved in cholesterol synthesis, including Hmgcr, Acss 2, Pmvk, Mvd, Fdft1, Ldlr and Sqle (Fig 2A). Elaidate did not induce expression of Srebp1. In fact, elaidate downregulated Srebp 1c (Fig 2A), and had minimal effect on the expression of SREBP1c target genes involved in fatty acid synthesis (Supplemental Fig 2). Consistent with the transcriptomics data, oleate downregulated the expression of the cholesterogenic genes, while palmitate showed a modest to no effect relative to control (Fig 2A). Similar effects of elaidate on cholesterogenic genes were observed in human Huh7 hepatoma cells (Fig 2B). 
A
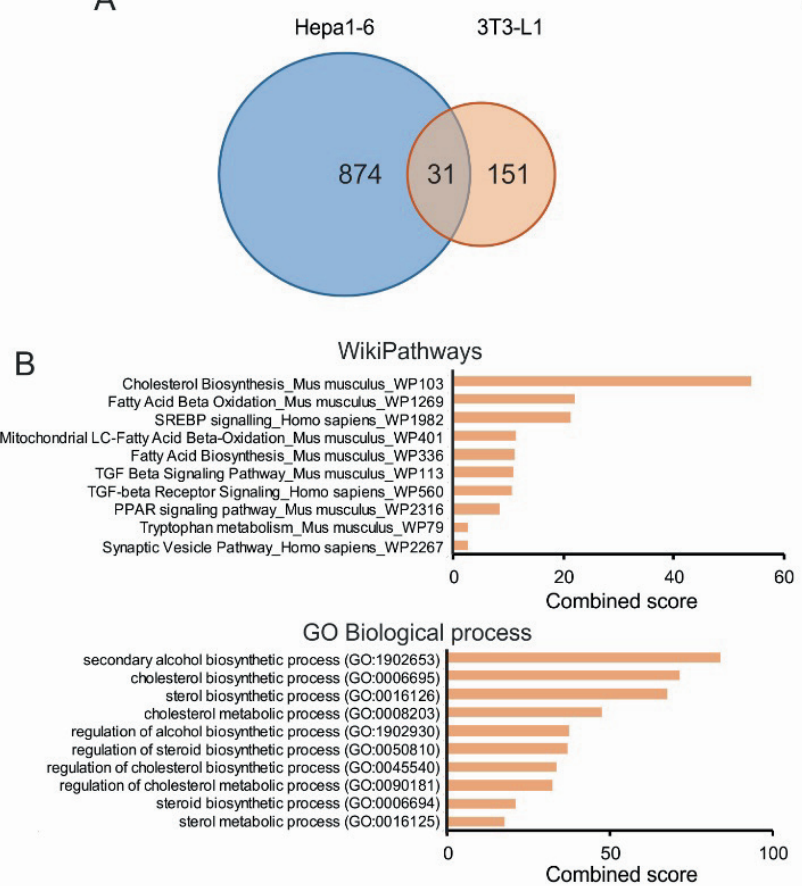

D

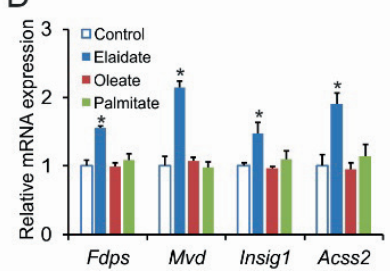

E
C

3T3-L1 Hepa1-6

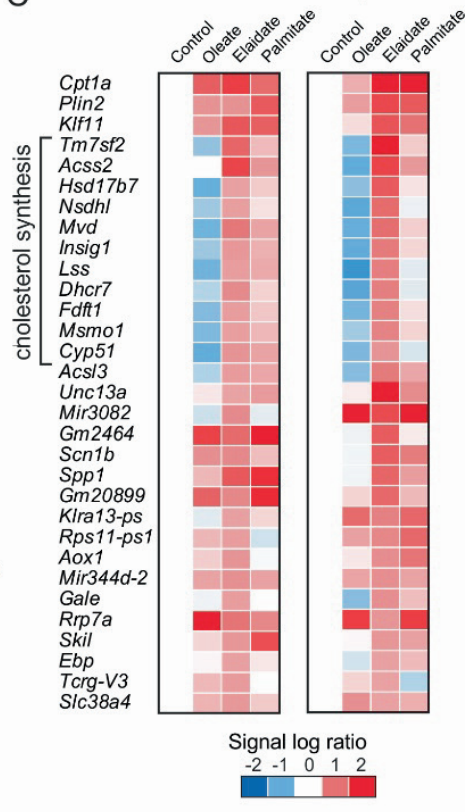

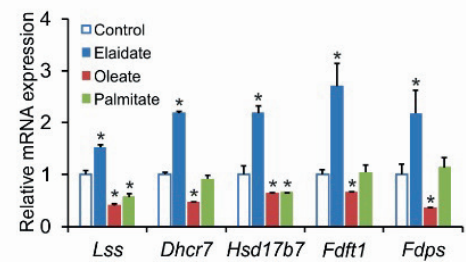

Figure 1. Elaidate induces a cholesterogenic expression profile in differentiated 3T3-L1 and

Hepa1-6 cells. Transcriptomics analysis was performed on differentiated 3T3-L1 treated with 1 $\mathrm{mM}$ fatty acids and Hepa1-6 cells treated with $500 \mu \mathrm{M}$ fatty acids for 24 hours. Genes with mean signal above 20 were selected. (A) Venn diagram showing number of upregulated genes by elaidate by at least 1.5-fold. (B) Pathway analysis by Enrichr of the 31 genes commonly upregulated by elaidate in differentiated 3T3-L1 and Hepa1-6 cells. (C) Heatmap showing relative expression profile of the 31 genes in differentiated 3T3-L1 and Hepa1-6 cells after treatment with elaidate, oleate or palmitate. (D-E) Relative mRNA expression by qPCR of cholesterol synthesis genes in 3T3-L1 adipocytes (D) and Hepa1-6 cells (E). mRNA expression was normalized to 36b4. Data are mean $\pm \mathrm{SD} ; * \mathrm{p}<0.05$ relative to control. 


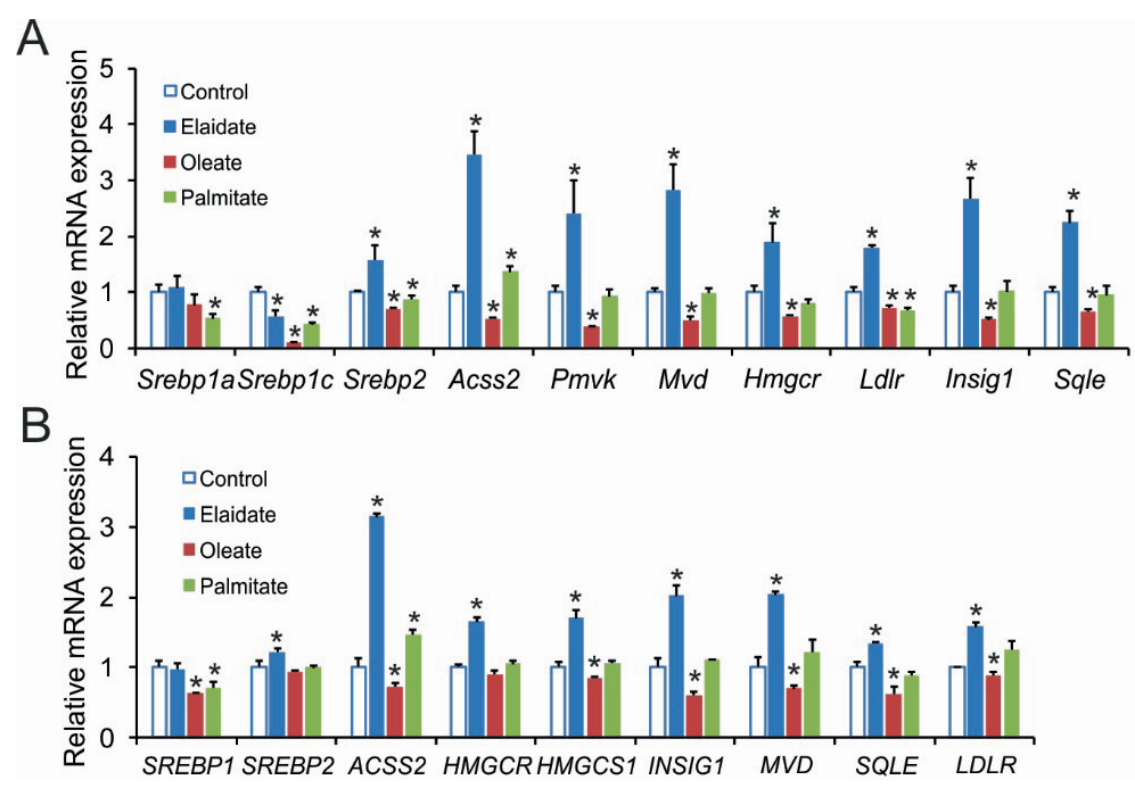

Figure 2. Induced expression of cholesterogenic genes in murine and human hepatoma cells by elaidate. (A) mRNA expression of cholesterogenic genes in Hepa1-6 cells treated with elaidate, oleate or palmitate at $500 \mu \mathrm{M}$ for 24 hours. (B) mRNA expression of cholesterogenic genes in Huh7 cells treated with elaidate, oleate or palmitate at $500 \mu \mathrm{M}$ for 24 hours. mRNA expression was normalized to $36 b 4$. Data are mean $\pm \mathrm{SD} ;{ }^{*} \mathrm{p}<0.05$ relative to control.

Guided by these results, we probed the role of SREBP1 and SREBP2 in mediating the induction of cholesterogenic genes by elaidate using siRNA. The siRNA-mediated silencing of Srebp1 and Srebp 2 completely abrogated the induction of cholesterogenic genes by elaidate (Fig 3A), supporting a role of SREBPs. Interestingly, expression of Srebp2 was not only reduced by siRNA-mediated silencing of Srebp2, but also by silencing of Srebpl (Fig 3A). Nevertheless, the observation that elaidate increased Srebp 2 mRNA but not Srebpl mRNA suggests that in these cells the induction of cholesterogenic genes by elaidate is likely mediated by SREBP 2 .

SREBP-dependent gene regulation requires SCAP for translocation to the Golgi. SCAP serves as a chaperone protein that facilitates the transport of SREBP to the Golgi apparatus for proteolytic processing and activation. Similar to the effect of Srebp1/Srebp2 silencing, silencing of Scap abrogated the induction of SREBP target genes by elaidate, indicating that the effects of elaidate require a functional SCAPSREBP axis (Fig 3B). The role of the SREBP/SCAP pathway in the regulation of cholesterogenesis by elaidate was further investigated at the protein level. Specifically, 
elaidate increased the levels of activated SREBP1 and 2, as well as the protein levels of SREBP targets HMGCR and ACSS2 (Fig 3C). Copying the mRNA data, knockdown of either isoforms of Srebp or Scap abolished the induction of ACSS2 and HMGCR by elaidate. Further in line with the mRNA data, silencing of Srebpl resulted in the absence of SREBP2, yet the opposite was not the case.

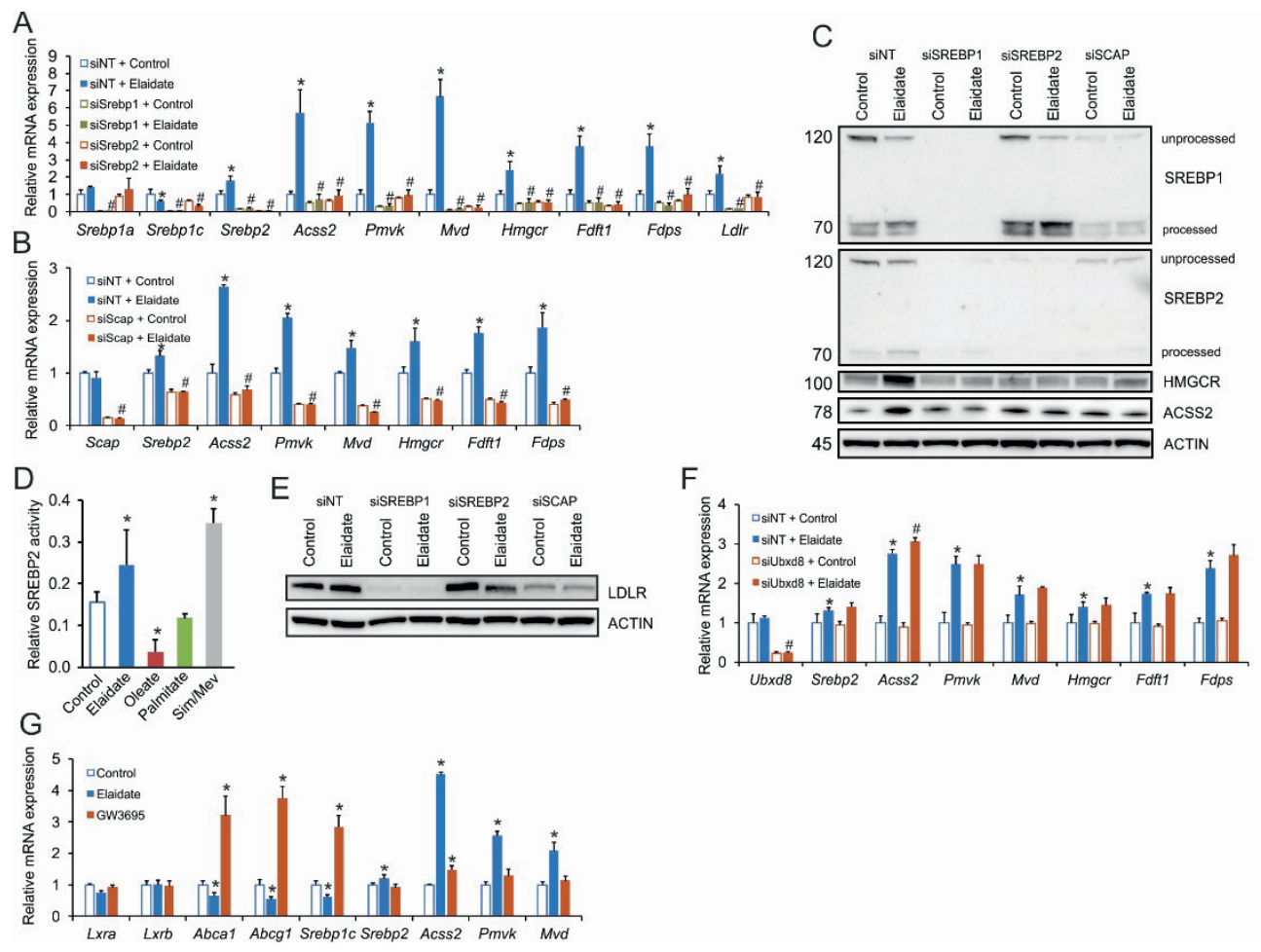

Figure 3. Elaidate induces cholesterogenic gene expression via SREBP2 and SCAP. (A) mRNA expression of cholesterogenic genes in Hepa1-6 cells treated with elaidate and siRNA against Srebp1 or Srebp2. (B) mRNA expression of cholesterogenic genes in Hepa1-6 cells treated with elaidate and siRNA against Scap. (C) Immunoblot for SREBP1, SREBP2, HMGCR and ACSS2 in Hepa1-6 cells treated with elaidate and siRNA against Srebp1, Srebp2 or Scap. $\beta$-Actin served as loading control. (D) Relative SREBP2 activity by PLAP reporter assay in HEK293 cells. Simvastain/mevalonic acid (Sim/Mev) at $5 \mu \mathrm{g} / \mathrm{mL}$ served as a positive control. (E) Immunoblot for LDLR in Hepa1-6 cells treated with elaidate and siRNA against Srebp1, Srebp 2 or Scap. $\beta$ Actin served as loading control. (F) mRNA expression of cholesterogenic genes in Hepa1-6 cells treated with elaidate and siRNA against $U b x d 8$. (G) mRNA expression of LXR and SREBP target genes in Hepa1-6 cells treated with elaidate or $1 \mu \mathrm{M}$ of the LXR agonist GW3695. mRNA expression was normalized to $36 \mathrm{~b} 4$. Data are mean $\pm \mathrm{SD} ;{ }^{*} \mathrm{p}<0.05$ relative to control; $\# \mathrm{p}<0.05$ relative to elaidate treatment with non-targeted siRNA (siNT + Elaidate). 
To further demonstrate that elaidate enhances the processing and activation of SREBP2, we performed a PLAP-reporter assay in HEK293, an assay that allows monitoring of translocation and subsequent processing of SREPB to its activate form [42]. In this assay, elaidate significantly enhanced the processing of SREBP2 to an extent comparable to that observed with statins (Fig 3D). By contrast, oleate decreased SREBP2 activity. Overall, our data suggest that the stimulatory effect of elaidate on cholesterogenic gene expression is mediated by the SREBP-SCAP pathway via SREBP2.

Intriguingly, despite upregulating Ldlr mRNA, elaidate did not affect LDLR protein (Fig 3E). Furthermore, elaidate reduced LDLR protein in Hepa1-6 cells treated with Srebp2 siRNA but not Srebpl or Scap siRNA (Fig 3E). These data suggest that, in addition to inducing cholesterogenic gene expression, elaidate has a suppressive effect on LDLR protein levels, which is independent of SREBP2 and dependent on SREBP1 and SCAP.

\section{Cholesterogenic effect of elaidate is independent of UBXD8 and LXR}

Having established that elaidate requires an intact SCAP-SREBP axis, we evaluated two potential mechanisms by which this fatty acid may stimulate SREBP signalling. First, we considered the possibility that elaidate requires UBXD8 for its activity, as previous studies have proposed this protein to serve as a sensor for unsaturated fatty acids [36]. UBXD8 is an ER membrane-associated protein that promotes SREBP processing by degrading INSIG1, an inhibitor of SREBP-SCAP translocation. However, as shown in Figure $3 \mathrm{~F}, U b x d 8 \mathrm{mRNA}$ was not affected by elaidate, nor did silencing of $U b d x d 8$ affect the induction of cholesterogenic genes by elaidate, suggesting that the induction is independent of UBXD8 (Fig 3F). Alternatively, we hypothesized that elaidate may inhibit activity of the Liver X receptors (LXR), which regulate Srebplc expression and which are reported to be inhibited by poly-unsaturated fatty acids [48]. To test this idea we treated Hepa1-6 cells with the LXR agonist GW3965. LXR activation markedly induced the expression of the established LXR target genes Abcal, Abcgl, and Srebplc, whereas elaidate suppressed these genes (Fig 3G). In turn, elaidate strongly induced cholesterogenic genes, whilst GW3965 had minimal to no effect. The above data do not support a role of UBXD8 and LXR in the activation of the SREBP-SCAP pathway by elaidate.

\section{Elaidate alters intracellular cholesterol metabolism}

To gain further insight into the mechanism behind the cellular effect of elaidate, we determined fluxes and metabolism of intracellular cholesterol. In the ER, cholesterol binds to SCAP to inhibit SREBP transport to the Golgi $[49,50]$. A previous study showed 
that elaidate upregulates SOAT1, the enzyme that catalyses cholesterol esterification [21]. Accordingly, we hypothesized that elaidate constitutively turns on SREBP signalling by promoting the esterification of cholesterol, thereby decreasing intracellular free cholesterol levels. While elaidate significantly reduced intracellular free cholesterol levels (Fig 4A), levels of cholesterol esters remained unaltered (Fig 4B). Furthermore, siRNA-mediated knockdown of Soatl did not abrogate the ability of elaidate to upregulate the expression of SREBP2 target genes (Fig 4C), indicating that the activation of the SCAP-SREBP pathway by elaidate is not dependent on cholesterol esterification.

A

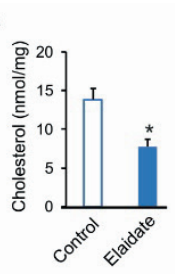

D
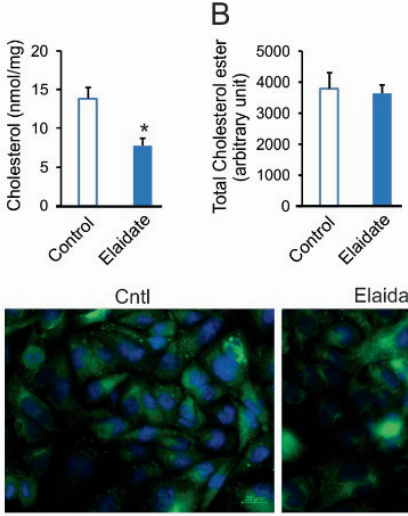

Elaidate

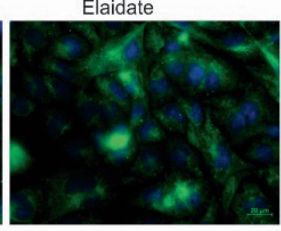

$\mathrm{F}$

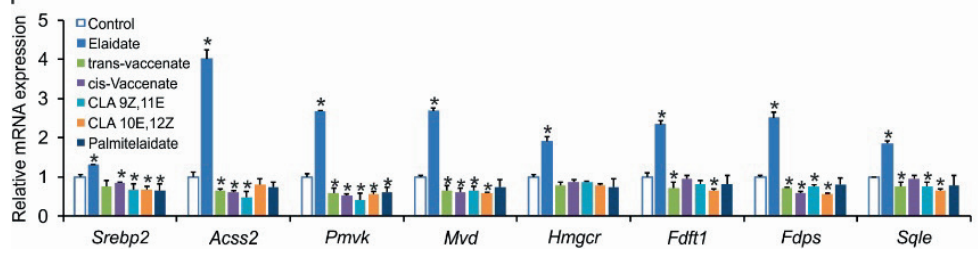

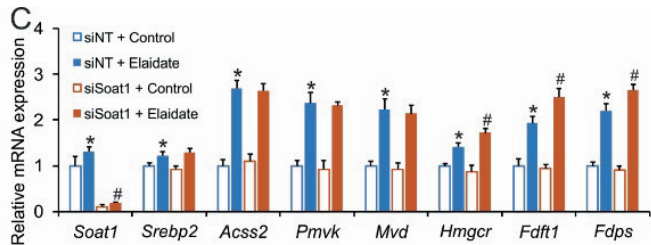

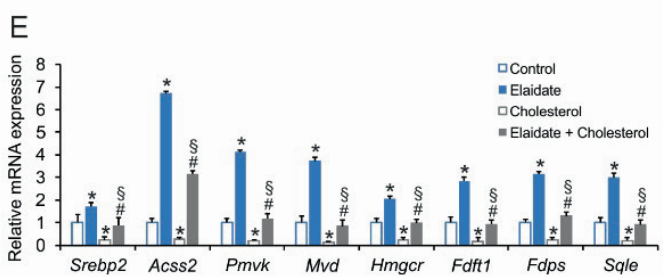

Figure 4. Elaidate alters intracellular cholesterol metabolism in Hepa1-6 cells. (A-B) Intracellular levels of free cholesterol (A) and esterified cholesterol (B) in Hepa1-6 cells treated with elaidate at $500 \mu \mathrm{M}$ for 24 hours. (C) mRNA expression of cholesterogenic genes in Hepa16 cells treated with elaidate and siRNA against Soat1. (D) SCAP localisation in SCAP-GFP expressing $\mathrm{CHO}$ cells treated with elaidate or control. (E) mRNA expression of cholesterogenic genes in Hepa1-6 cells treated for 24 hours with $500 \mu \mathrm{M}$ elaidate and/or $500 \mu \mathrm{M}$ of exogenous cholesterol. (F) mRNA expression of cholesterogenic genes in Hepa1-6 cells treated with $500 \mu \mathrm{M}$ of elaidate or other trans fatty acids. mRNA expression was normalized to $36 \mathrm{~b} 4$. Images are $200 \mathrm{x}$ magnification. Data are mean $\pm \mathrm{SD} ;{ }^{*} \mathrm{p}<0.05$ relative to control; $\# \mathrm{p}<0.05$ relative to elaidate or elaidate treatment with non-targeted siRNA (siNT + Elaidate); $\S p<0.05$ relative to cholesterol treatment. 
Next, we considered the possibility that elaidate directly influences SCAP translocation. To test this, we studied CHO cells that stably produce SCAP-GFP [43]. In these cells, treatment with elaidate did not change the cellular distribution of SCAP (Fig 4D). Alternatively, elaidate may modulate cholesterol sensing by SCAP. To test this notion, we increased intracellular levels of cholesterol by delivering exogenous cholesterol, either in the absence or presence of elaidate. As expected, elaidate induced cholesterogenic gene expression, while exogenous cholesterol had a marked suppressive effect on the same set of genes (Fig 4E). In the presence of elaidate, exogenous cholesterol still significantly reduced cholesterogenic gene expression, suggesting that elaidate does not completely abolish the cholesterol-sensing ability of SCAP. However, compared to the cholesterol-only condition, adding elaidate significantly upregulated cholesterogenic gene expression, suggesting that elaidate desensitizes SCAP to cholesterol (Fig 4E). Taken together, these results suggest that elaidate activates the SREBP/SCAP pathway through complementary mechanisms that include lowering intracellular free cholesterol levels and desensitizing SCAP to cholesterol.

Finally, we examined whether the stimulatory effect of elaidic acid on cholesterogenic genes can be generalized to dietary trans fatty acids as a group. Remarkably, elaidate has distinct effects from natural trans fatty acids. Whereas the industrially-produced elaidate upregulated cholesterol synthesis genes, natural trans fatty acids such as trans-vaccenate, conjugated linoleate, and palmitelaidate did not show such an effect (Fig 4F). In fact, if anything, these naturally occurring trans fats mildly decreased expression of these genes. Our data thus indicate that elaidate, but not ruminant trans fatty acids, activate the SREBP-SCAP pathway in cultured hepatoma cells.

\section{Trans fat feeding causes lipoatrophy and hepatomegaly in mice}

In a separate set of studies, we aimed to investigate the effects of industrial trans fatty acids on the liver in vivo. To that end, male C57B1/6 mice were randomly assigned to one of 3 calorically equivalent diets enriched in different types of fatty acids: a Trans diet, a Cis diet, or a Saturated diet. The Trans diets consisted of 30-35\% trans-unsaturated fatty acids originating from partially hydrogenated soybean oil (Fig 5A, Supplemental Table 3). The Cis diet contained equivalent amount of saturated fatty acids as the Trans diet, but with cis mono- and poly-unsaturated fatty acids replacing the trans-unsaturated fatty acids. The Saturated diet contained about $60 \%$ saturated fatty acids, but with equivalent amounts of cis mono- and poly-unsaturated fatty acids as the Trans diet. Overall, about $60-65 \%$ of the fatty acid composition was similar between the three diets, with the remaining $35-40 \%$ being taken up by either trans-unsaturated fatty acids, cis- 
unsaturated fatty acids or saturated fatty acids (Fig 5A). Food intake (Fig 5B) and body weight (Fig 5C) were not significantly different between the 3 groups of mice. In comparison to the mice fed the Saturated and Cis diet, the mice fed the Trans diet had a significantly higher relative liver weight (Fig. 5D), but significantly lower relative gonadal fat weight (Fig 5E). Consequently, the ratio of liver to gonadal fat weight was approximately 2-fold higher in the mice fed the Trans diet (Fig 5F). No significant differences in plasma levels of cholesterol, glycerol, non-esterified fatty acids, glucose, and insulin were observed between the Trans group and the Cis and Saturated groups (Fig 5G).
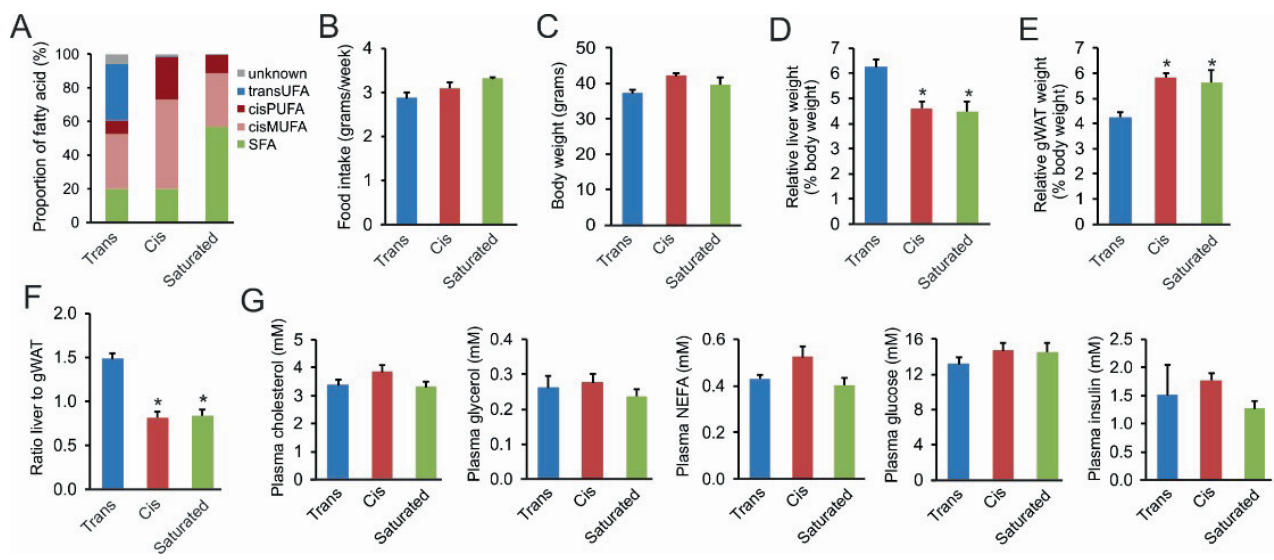

Figure 5. Anthropometric measurements and plasma metabolites in mice fed the different high fat diets for 7 weeks. (A) Profile of relative content of different fatty acids in the test diets. TFA, trans-unsaturated fatty acids; PUFA, poly-unsaturated fatty acids; cisMUFA, cis monounsaturated fatty acids; SFA, saturated fatty acids. (B) Average weekly food intake during the 7week diet intervention. (C) Final body weights. (D) Relative liver weight. (E) Relative weight of gonadal white adipose tissue (gWAT). (F) Ratio of liver to gWAT weight. (G) Plasma levels of cholesterol, glycerol, non-esterified fatty acids (NEFA), glucose, and insulin. Data are mean \pm $\mathrm{SEM} ; \mathrm{N}=8$ mice/group; ${ }^{*} \mathrm{p}<0.05$ relative to Trans group.

\section{Trans fat feeding promotes non-alcoholic fatty liver disease}

Remarkably, plasma alanine aminotransferase activity was significantly higher in the Trans group than in the Cis and Saturated groups, suggesting enhanced liver damage (Fig 6A). In addition, plasma levels of the acute phase proteins haptoglobin (Fig 6B) and serum amyloid A (Fig 6C) were markedly higher in the mice fed the Trans diet. Furthermore, hepatic triglycerides and cholesterol levels were higher in the Trans group compared to the Saturated group (Fig 6D and E). Supporting the quantitative triglyceride 
analysis, H\&E staining revealed enhanced steatosis in the livers of mice fed the Trans diet in comparison with mice fed the Saturated and Cis diets (Fig 6F).
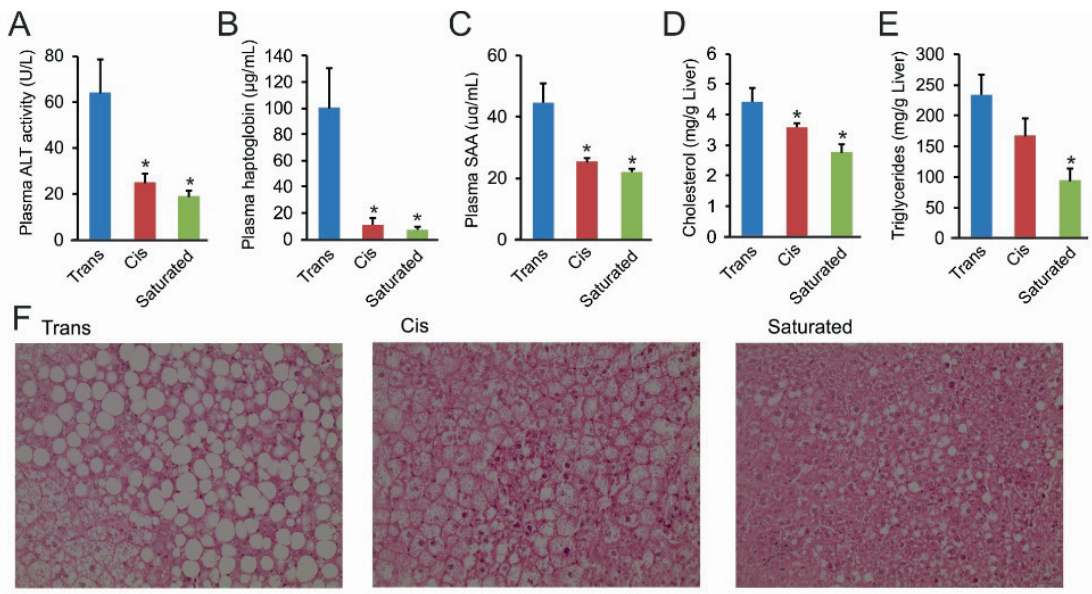

G

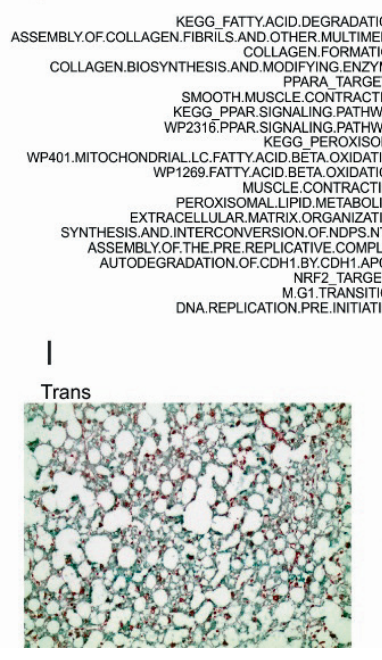

row

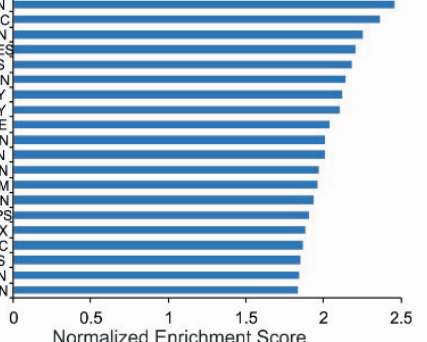

$\mathrm{H}$
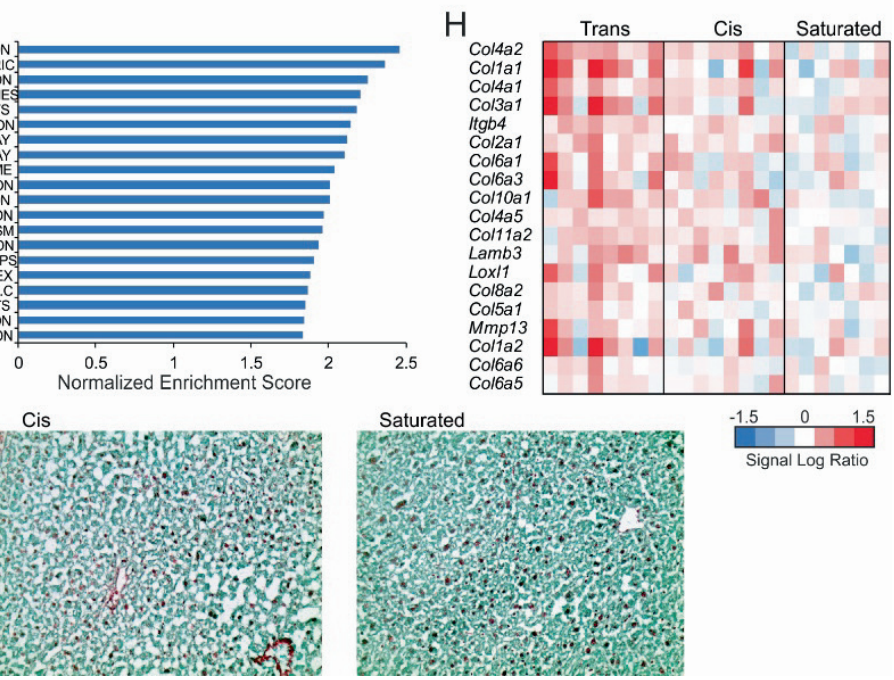

Figure 6. Increased liver damage, steatosis and fibrosis in mice fed the Trans diet. (A) Plasma levels of alanine aminotransferase (ALT) activity. (B-C) Plasma levels of the acute phase proteins haptoglobin (B) and serum amyloid A (C). (D-E) Quantification of hepatic content of cholesterol (D) and triglycerides (E). (F) Representative images of liver steatosis by haematoxylin \& eosin staining. (G) Top 20 upregulated gene sets in livers of mice fed the Trans diet, determined by gene set enrichment analysis. Gene sets were ranked according to normalized enrichment score. (H) Expression profile of genes within the gene set Assembly of Collagen Fibrils. (I) Representative images of collagen staining using Fast Green FCF/Sirius Red. Images are 200x magnification. Data are mean $\pm \mathrm{SEM} ; \mathrm{N}=8$ mice/group; ${ }^{*} \mathrm{p}<0.05$ relative to Trans group. 

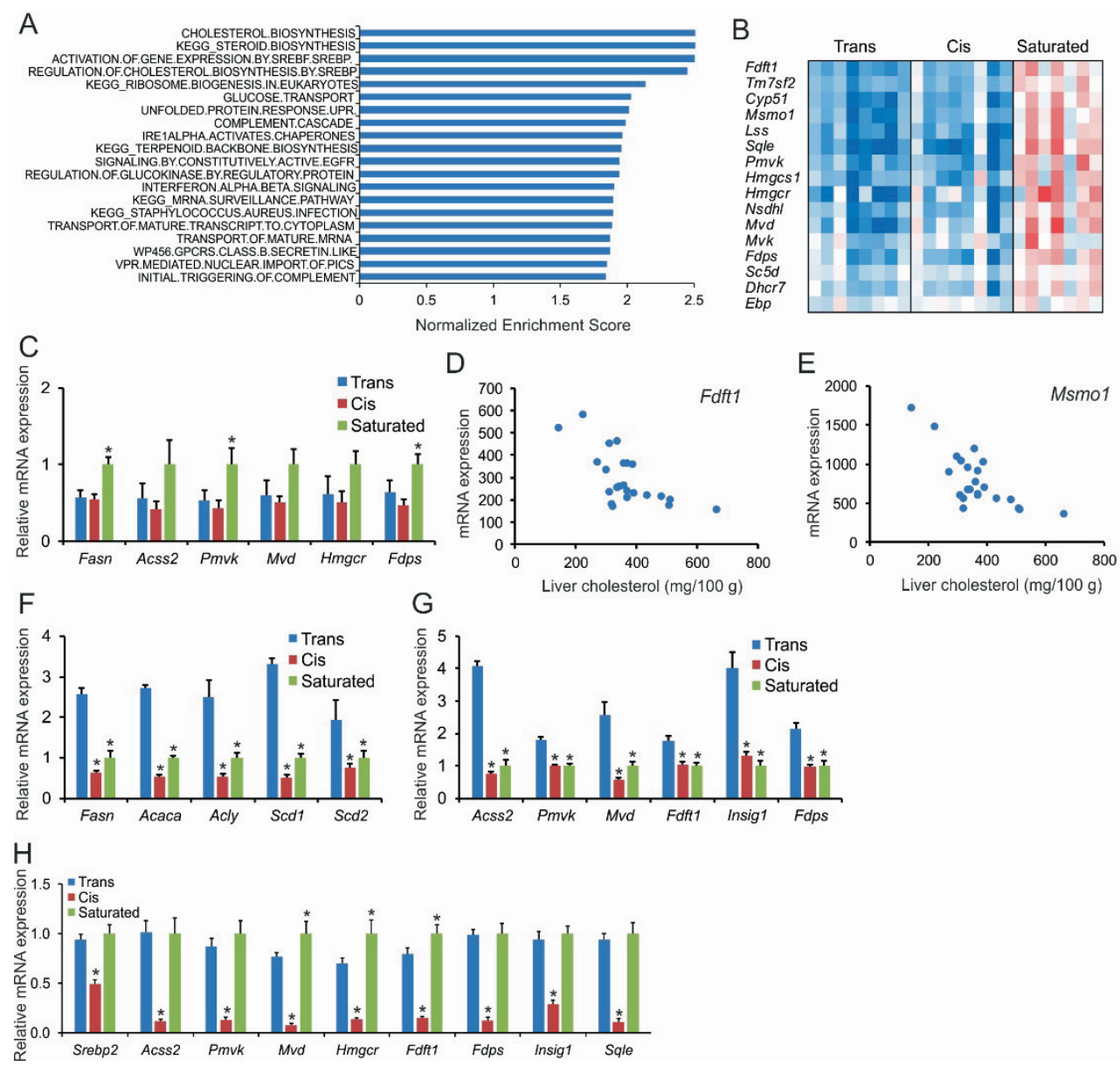

Figure 7. Reduced cholesterogenic gene expression in livers of mice fed the Trans diet. (A) Top 20 downregulated gene sets in livers of mice fed the Trans diet for 7 weeks, determined by gene set enrichment analysis. Gene sets were ranked according to normalized enrichment score. (B) Expression profile of genes within the gene set Cholesterol Biosynthesis. (C) Relative mRNA expression by qPCR of cholesterol synthesis genes in the liver of mice fed the 3 different types of diets. (D-E) Strong negative correlation between hepatic cholesterol levels and liver expression of the cholesterol synthesis genes Fdft1 (D) and Msmol (E) in the mice fed the Trans diet. (F-G) Relative mRNA expression of fatty acid synthesis genes (F) and cholesterol synthesis genes $(\mathrm{G})$ in the gonadal adipose tissue of mice fed the 3 different types of diets. (H) mRNA expression of cholesterogenic genes in liver of mice fed overnight with the Trans, Cis or Saturated diets. mRNA expression was normalized to $36 \mathrm{b4}$. Data are mean \pm SEM. $\mathrm{N}=8$ mice/group for the chronic feeding study and $\mathrm{N}=10$ mice/group for the overnight feeding study. ${ }^{*} \mathrm{p}<0.05$ relative to Trans group. 
To further characterize the hepatic phenotype in the three groups, we performed transcriptomics analysis. Gene set enrichment analysis showed elevated expression of collagen formation pathways and fatty acid degradation pathways in the mice fed the Trans diet (Fig 6G). The higher expression of collagen-related genes in the Trans group compared to the Cis and Saturated group is visualized in Fig 6H. Consistent with the transcriptomic results, hepatic collagen staining was more pronounced in the mice fed the Trans diet (Fig 6I), further supportive of a more marked NAFLD phenotype.

Interestingly, gene set enrichment analysis showed decreased expression of the cholesterol synthesis pathway in the mice fed the Trans and Cis diet compared to the Saturated fat (Fig 7A, B), which was confirmed by qPCR (Fig 7C). The expression of cholesterol synthesis genes was highly negatively correlated with hepatic cholesterol levels, suggesting that cholesterogenic gene expression is mainly determined by hepatic cholesterol levels (Fig 7D, E). In contrast to liver, in gonadal adipose tissue the expression of genes involved in lipogenesis (Fig 7F) and cholesterogenesis (Fig 7G) was markedly higher in the Trans group than in the Cis and Saturated group. This lipo- and cholesterogenic effect of the Trans diet was recapitulated in the inguinal adipose tissue depot (Supplemental Fig 3). Taken together, these data show that the Trans diet led to more advanced NALFD as compared to the Cis and Saturated diet, as shown by elevated alanine aminotransferase activity, elevated triglyceride and cholesterol levels, and increased expression of fibrosis-related genes.

Since lower cholesterogenic gene expression in the liver after chronic high fat feeding seemed to be mainly driven by higher hepatic cholesterol levels, we determined the acute effect of the different diets on hepatic gene expression. To this end, we fed mice overnight with the Trans diet, the Cis diet, or the Saturated diet. Interestingly, cholesterogenic gene expression was markedly higher in the mice fed the Trans diet compared to mice fed the Cis diet (Fig 7H). In addition, the expression of cholesterogenic genes was highly similar between the Saturated and the Trans group, suggesting that in vivo the acute effects of industrial trans and saturated fatty acids are similar. 


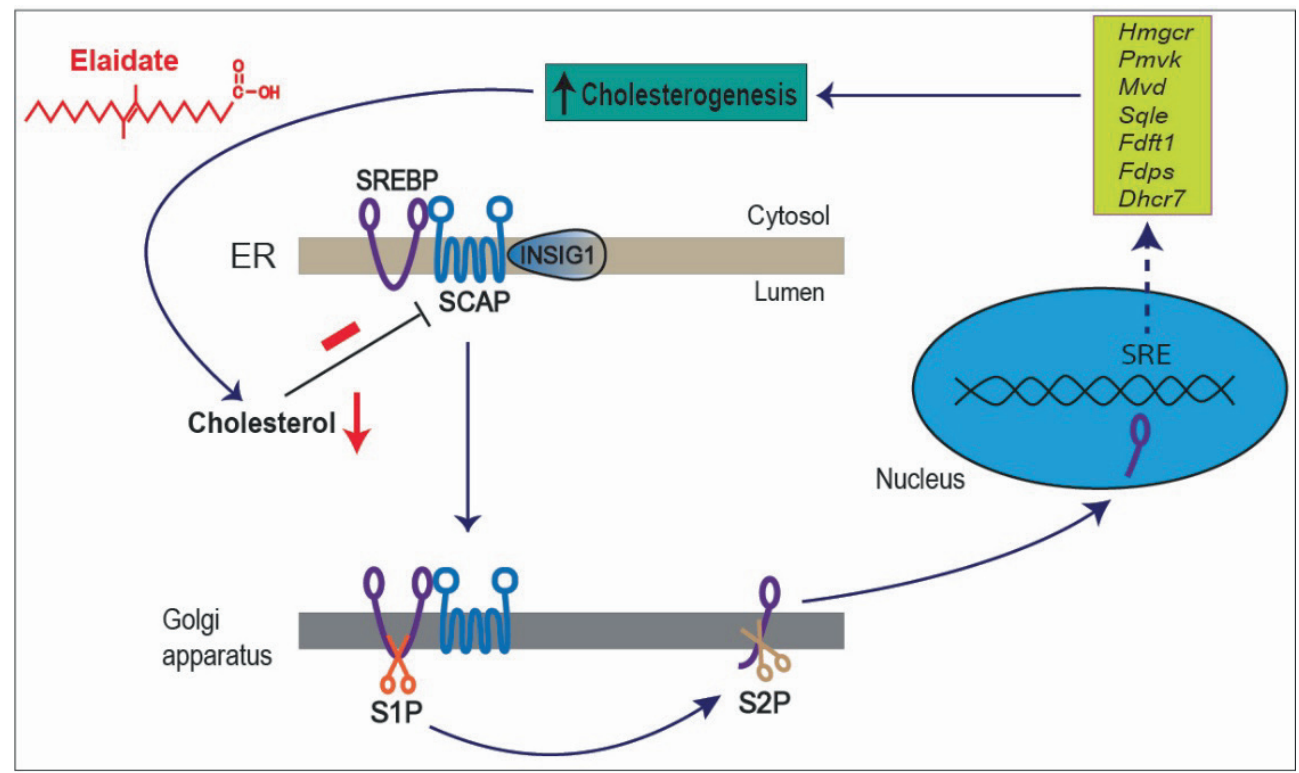

Figure 8: Graphical abstract: Potential mechanism of elaidate. Elaidate activates the SREBP signalling pathway by reducing the sensitivity of SCAP to cholesterol and lowering the levels of intracellular cholesterol. 


\section{Discussion}

The major outcomes of our studies are: 1) in vivo, a diet enriched in industrial trans fatty acids promotes NAFLD when compared with a diet enriched in cis-unsaturated and saturated fatty acids. Specifically, we find that feeding mice a diet enriched in industrial trans fatty acids enhances liver steatosis and fibrosis, and raises hepatic triglyceride levels, hepatic cholesterol levels, and plasma alanine aminotransferase activity in comparison with diets enriched in cis-unsaturated or saturated fatty acids. 2) in vitro, the industrial trans fatty acid elaidate, but not ruminant trans fatty acids, stimulates the cholesterol synthesis pathway in liver cells via activation of the SCAP/SREBP2 axis, presumably by lowering intracellular free cholesterol and desensitizing SCAP to cholesterol.

Using an integrated transcriptomics, lipidomics, and proteomics approach in HepG2 cells, it was previously shown that elaidate induces the expression of several genes involved in fatty acid and cholesterol synthesis [21]. Here, using siRNA-mediated gene silencing, we show that the cholesterogenic effect of elaidate is dependent on functional SREBP2 and SCAP. Although silencing of Srebp1 abolished the induction of cholesterogenic genes by elaidate, the role of SREBP1 is probably indirect by serving as an important transcriptional regulator of SREBP2. Indeed, SREBP2 protein and mRNA were nearly equally silenced by siRNA targeting Srebp 2 and Srebp1. Accordingly, the abrogation of the elaidate effect by Srebpl silencing is likely due to the concomitant downregulation of SREBP2. This result is in line with studies that show that both SREBP1 and SREBP2 contain a sterol response element (SRE) in their respective promoter regions, which mediates upregulation of the two isoforms in a feed-forward manner [51,52]. Also, the reduction in protein levels of SREBP1 and SREBP2 after SCAP knockdown agrees with a previous study that reported profound reductions in mRNA and protein levels of SREBP1 and SREBP2 in mice with liver-specific deletion of SCAP, due in part to the abrogation of the feed-forward mechanism that enables SREBPs to regulate their own expression. [53].

It is well established that the SREBP/SCAP pathway is controlled by fatty acids. Evidence indicates that (poly)-unsaturated fatty acids inhibit SREBP1-induced lipogenesis in the liver by suppressing SREBP1 mRNA levels and inhibiting proteolytic processing of SREBP1. The direct target of (poly)-unsaturated fatty acids was proposed to be UBXD8, an ER membrane-bound protein that facilitates the degradation of INSIG1, which normally sequesters the SCAP-SREBP complex in the ER and prevents its activation [36,37]. Our data indicate that activation of the SREBP/SCAP pathway by 
elaidate is independent of UBXD8. Our data also indicate that the effect of elaidate is distinct from that of oleate. Whereas oleate suppressed the expression of cholesterogenic genes in Hepa1-6 and 3T3-L1 cells, elaidate had the opposite effect. Apparently, the configuration of the double bond in 9-octadecenoic acid dramatically alters its signalling properties in liver and other cells. The effect of palmitate was intermediate between that of oleate and elaidate in Hepa1-6 cells, and diverges from elaidate in 3T3-L1 cells, supporting our previous report that in macrophages, elaidate and palmitate have distinct signalling properties [20].

As already indicated, elevated levels of intracellular free cholesterol serves as a negative feedback regulator of SREBP signalling. When intracellular cholesterol levels drop, the interaction between SREBP-SCAP and INSIG1 is released, triggering the movement of SREBP-SCAP to the Golgi, where SREBP becomes activated [54,55]. We found that elaidate significantly reduced intracellular free cholesterol levels, likely accounting for the activation of the SREBP/SCAP pathway. One possible explanation for the lower free cholesterol levels is enhanced cholesterol esterification. Although Soat1 has been reported to be induced by elaidate [21], silencing of Soatl did not abrogate the cholesterogenic effects of elaidate, nor did elaidate increase intracellular levels of cholesterol ester, ruling out enhanced cholesterol esterification as key underlying mechanism. Besides lowering intracellular free cholesterol, elaidate partially but significantly repressed the strong anti-cholesterogenic effect of exogenous cholesterol, suggesting that elaidate decreases the sensitivity of SCAP to cholesterol. Overall, our data suggest that elaidate activates the SREBP signalling through complementary mechanisms that include lowering intracellular free cholesterol levels and desensitizing SCAP to cholesterol.

Previously, Koppe and colleagues showed that compared to diets high in saturated fatty acids, a diet high in industrial trans fatty acids markedly increased plasma alanine aminotransferase activity in AKR/J mice, suggesting enhanced liver damage [56]. Furthermore, studies in different mouse strains revealed that diets rich in industrial trans fatty acid from partially hydrogenated vegetable oil promote hepatic steatosis and fibrosis $[17,57,58]$. Our in vivo study, which provides the most detailed characterization of the effects of industrial trans fatty acids on the liver, extends these findings by showing that a diet enriched in trans fatty acids enhances liver steatosis and fibrosis, and raises hepatic triglyceride levels, hepatic cholesterol levels, plasma acute phase proteins, and plasma alanine aminotransferase activity. Accordingly, we now have convincing evidence that industrial trans fatty acids promote NALFD in mice. 


\section{Chapter 3}

Our study is also consistent with other studies showing that the trans fatty acid-induced increase in liver mass and hepatic lipid accumulation is accompanied by a decrease in fat tissue mass $[15,59,60]$. One potential explanation for the pronounced increase in the liver to fat ratio is that trans fatty acids may be preferentially directed towards the liver at the expense of fat tissue. Currently, it is unclear how this can happen. It can be hypothesized that lipoproteins containing triglycerides enriched in trans fatty acids may be less efficiently hydrolyzed by lipoprotein lipase in fat tissue. As a result, a larger portion of the dietary fat is taken to the liver. Unfortunately, there are no tools to experimentally validate this hypothesis, given the lack of radiolabelled trans fatty acids and triglycerides. An alternative explanation is that differences in liver to fat ratio are related to differences in adipose tissue lipolysis. However, plasma levels of NEFA and glycerol were not significantly different between the groups, suggesting that adipose tissue lipolysis is not different between the groups. Although it would certainly be a worthwhile pursuit to tease out the processes underlying the differential in vivo partitioning of trans fatty acids, tools are lacking to properly address this question.

Our studies also do not allow us to draw conclusions about whether trans fatty acids themselves are responsible for the enhanced NASH features or whether the effects could be mediated by the increase in hepatic cholesterol. Indeed, emerging evidence suggests that hepatic free cholesterol is a major lipotoxic molecule that plays a critical role in the development of NASH $[61,62]$. Taking into account the damaging role of cholesterol, it is not unreasonable to suggest an increase in cholesterogenesis may underlie the stimulation of NASH by industrial trans fatty acids.

Given the major differences in experimental set-up and complexity of the treatments between the in vitro and in vivo studies, one should be careful to directly compare the outcomes of the two experimental approaches. Nevertheless, cis-unsaturated fatty acids seemed to behave similarly in vivo and in vitro, as the Cis diet and oleate led to lower cholesterogenic gene expression compared to the Saturated diet and palmitate, respectively. By contrast, whereas elaidate induced cholesterogenic gene expression in Hepa1-6 cells in comparison with palmitate, chronic feeding of the Trans diet reduced cholesterogenic gene expression in liver in comparison with the Saturated diet, while overnight feeding of the Trans diet led to similar cholesterogenic gene expression as the Saturated diet. Compared with oleate, elaidate induced cholesterogenic gene expression in vitro, and overnight feeding of the Trans diet led to higher cholesterogenic gene expression than the Cis diet. Intriguingly, chronic feeding of the Trans and the Cis diet led to similar cholesterogenic gene expression. Although caution should be exercised when comparing the outcomes of in vitro and in vivo approaches, it is tempting to 
attribute the increase in hepatic cholesterol in the Trans group to activation of the SREBP/SCAP pathway. It can be speculated that the Trans diet initially stimulated hepatic cholesterogenesis, leading to the observed elevation in liver cholesterol levels, which in turn caused a compensatory decrease in cholesterogenic gene expression. Alternatively, it is possible that the increase in liver triglyceride and cholesterol levels in the Trans group is caused by preferential shuttling of circulating lipids to the liver.

Epidemiological studies have found a positive association between intake of trans fatty acids and NAFLD [12,13]. Our and other animal studies suggest that this association may be causal. Currently, there is no record of a nutritional trial that investigated the effect of industrial trans fatty acids on intrahepatic lipid or markers of liver damage in humans. It is likely that these studies will never be performed due to ethical restrictions. Industrial trans fatty acids are best known for their ability to raise plasma LDL cholesterol and lower plasma HDL cholesterol levels in human volunteers $[6,63,64]$. Due to the very different lipoprotein profiles of mice and humans, effects of specific treatments on plasma lipid levels in mice cannot easily be extrapolated to the human situation. Nevertheless, we anticipated that the molecular mechanism of action of elaidate in liver cells might give some clues about the plasma cholesterol-modulating effect of trans fatty acids. The observed activation of the SREBP/SCAP pathway by elaidate is expected to enhance the extraction of LDL from the bloodstream by activating the transcription of LDLR. Indeed, elaidate significantly increases Ldlr mRNA in Hepa1-6 cells, which is mediated by a functional SREBP/SCAP pathway. Strikingly, elaidate was found to suppress LDLR protein levels, via a mechanism independent of SREBP2 and dependent on SREBP1. One possibility is that elaidate decreases LDLR protein by enhancing its degradation. Based on these data, it could be hypothesized that SREBP1 drives the expression of a gene that mediates the degradation of LDLR by elaidate. One obvious candidate would be Pcsk9. However, mRNA expression levels of $P c s k 9$ were not altered by elaidate. Additional studies are necessary to elucidate the mechanism accounting for the suppressive effect of elaidate on LDLR protein.

In conclusion, we show that industrial trans fatty acids stimulate cholesterogenesis in vitro via activation of the SREBP/SCAP pathway. Induction of cholesterogenesis might explain the increase in liver cholesterol and NAFLD in mice fed a diet rich in transunsaturated fatty acids. 
Acknowledgments: All authors edited and revised the manuscript and approved the final version. Sander Kersten $(\mathrm{PhD})$ is responsible for the integrity of the work as a whole.

We would like to thank Dominique Massau and Imke Vohs for their assistance in the lab, and their help in generating some of the in vitro data.

Funding: This project was funded by the Graduate School, VLAG from Wageningen University, The Netherlands.

Noam Zelcer is an Established Investigator of the Dutch Heart Foundation (2013T111) and is supported by an ERC Consolidator grant (617376) from the European Research Council and by a Vici grant from the Netherlands Organization for Scientific Research (NWO; 016.176.643).

Disclosure: The authors declare no conflict of interest. 


\section{References}

[1] Kummerow, F.A., 2009. The negative effects of hydrogenated trans fats and what to do about them. Atherosclerosis 205(2): 458-65.

[2] Filip, S., Vidrih, R., 2008. Trans Fatty Acids and Human Health 16.

[3] Stender, S., Astrup, A., Dyerberg, J., 2008. Ruminant and industrially produced trans fatty acids: Health aspects. Food and Nutrition Research 52: 1-8.

[4] Brouwer, I.A., Wanders, A.J., Katan, M.B., 2010. Effect of animal and industrial Trans fatty acids on HDL and LDL cholesterol levels in humans - A quantitative review. PLoS ONE 5(3): 1-10.

[5] Chardigny, J., Destaillats, F., Malpuech-brugère, C., Moulin, J., Bauman, D.E., Lock, A.L., et al., 2008. Do trans fatty acids from industrially produced sources and from natural sources have the same effect on cardiovascular disease risk factors in healthy subjects? Results of the trans Fatty Acids Collaboration ( TRANSFACT ) study $1-$ 4 (May): 558-66.

[6] Mensink, R.P.\&., Katan, M.B., 1990. Effect of dietary trans fatty acids on highdensity and low-density lipoprotein cholesterol levels in healthy subjects. New England Journal of Medicine 327(2): 82-7.

[7] Remig, V., Franklin, B., Margolis, S., Kostas, G., Nece, T., Street, J.C., 2010. Trans Fats in America: A Review of Their Use, Consumption, Health Implications, and Regulation. Journal of the American Dietetic Association 110(4): 585-92.

[8] Mozaffarian, D., Katan, M.B., Ascherio, A., Stampfer, M.J., Willett, W.C., 2006. Trans Fatty Acids and Cardiovascular Disease. New England Journal of Medicine 354(15): 1601-13.

[9] Ganguly, R., Pierce, G.N., 2012. Trans fat involvement in cardiovascular disease. Molecular Nutrition and Food Research 56(7): 1090-6.

[10] Ganguly, R., Pierce, G.N., 2015. The toxicity of dietary trans fats. Food and Chemical Toxicology 78: 170-6.

[11] de Souza, R.J., Mente, A., Maroleanu, A., Cozma, A.I., Ha, V., Kishibe, T., et al., 2015. Intake of saturated and trans unsaturated fatty acids and risk of all cause mortality, cardiovascular disease, and type 2 diabetes: systematic review and metaanalysis of observational studies. BMJ (Clinical Research Ed.) 351(6).

[12] Mazidi, M., Katsiki, N., Mikhailidis, D.P., Banach, M., 2018. Link between plasma trans-fatty acid and fatty liver is moderated by adiposity. International Journal of Cardiology 272: 316-22.

[13] Mantovani, A., 2018. Plasma trans-fatty acid and risk of nonalcoholic fatty liver disease: New data from National Health and Nutrition Examination Survey (NHANES). International Journal of Cardiology 272: 329-30.

[14] Sullivan, S., 2010. Implications of diet on nonalcoholic fatty liver disease. Current Opinion in Gastroenterology 26(2): 160-4. 
[15] Machado, R.M., Stefano, J.T., Oliveira, C.P.M.S., Mello, E.S., Ferreira, F.D., Nunes, V.S., et al., 2010. Intake of trans fatty acids causes nonalcoholic steatohepatitis and reduces adipose tissue fat content. The Journal of Nutrition 140(6): 1127-32.

[16] Obara, N., Fukushima, K., Ueno, Y., Wakui, Y., Kimura, O., Tamai, K., et al., 2010. Possible involvement and the mechanisms of excess trans-fatty acid consumption in severe NAFLD in mice. Journal of Hepatology 53(2): 326-34.

[17] Hu, X., Tanaka, N., Guo, R., Lu, Y., Nakajima, T., Gonzalez, F.J., et al., 2017. PPAR $\alpha$ protects against trans-fatty-acid-containing diet-induced steatohepatitis. Journal of Nutritional Biochemistry 39: 77-85.

[18] Collison, K.S., Maqbool, Z., Saleh, S.M., Inglis, A., Makhoul, N.J., Bakheet, R., et al., 2009. Effect of dietary monosodium glutamate on trans fat-induced nonalcoholic fatty liver disease. Journal of Lipid Research 50(8): 1521-37.

[19] Zhao, X., Shen, C., Zhu, H., Wang, C., Liu, X., Sun, X., et al., 2016. Trans-fatty acids aggravate obesity, insulin resistance and hepatic steatosis in C57BL/6 mice, possibly by suppressing the IRS1 dependent pathway. Molecules 21(6): 1-11.

[20] Oteng, A.-B., Bhattacharya, A., Brodesser, S., Qi, L., Tan, N.S., 2017. Feeding Angptl4- / - mice trans fat promotes foam cell formation in mesenteric lymph nodes without leading to ascites. J. Lipid Res. 58: 1-36.

[21] Vendel Nielsen, L., Krogager, T.P., Young, C., Ferreri, C., Chatgilialoglu, C., Nørregaard Jensen, O., et al., 2013. Effects of Elaidic Acid on Lipid Metabolism in HepG2 Cells, Investigated by an Integrated Approach of Lipidomics, Transcriptomics and Proteomics. PLoS ONE 8(9).

[22] Shao, F., Ford, D.A., 2014. Elaidic acid increases hepatic lipogenesis by mediating sterol regulatory element binding protein-1c activity in HuH-7 cells. Lipids 49(5): 403-13.

[23] Hua, X., Wu, J., Goldstein, J.L., Brown, M.S., Hobbs, H.H., 1995. Structure of the human gene encoding sterol regulatory element binding protein-1 (SREBF1) and localization of SREBF1 and SREBF2 to chromosomes 17p11.2 and 22q13. Genomics 25(3): 667-73.

[24] Yokoyama, C., Wang, X., Briggs, M.R., Admon, A., Wu, J., Hua, X., et al., 1993. SREBP-1, a basic-helix-loop-helix-leucine zipper protein that controls transcription of the low density lipoprotein receptor gene. Cell 75(1): 187-97.

[25] Brown, M., Goldstein, J., 1997. SREBP pathway: regulation of cholesterol metabolism by proteolysis of membrane bound transcription factor. Cell 89(1): 33140 .

[26] Amemiya-Kudo, M., Shimano, H., Hasty, A.H., Yahagi, N., Yoshikawa, T., Matsuzaka, T., et al., 2002. Transcriptional activities of nuclear SREBP-1a, -1c, and -2 to different target promoters of lipogenic and cholesterogenic genes. Journal of Lipid Research 43(8): 1220-35.

[27] Horton, J.D., Goldstein, J.L., Brown, M.S., 2002. Critical review. Most 109(9): 1125-31. 
[28] Goldstein, J.L., DeBose-Boyd, R.A., Brown, M.S., 2006. Protein sensors for membrane sterols. Cell 124(1): 35-6.

[29] Steck, T.L., Lange, Y., 2002. SCAP, an ER sensor that regulates cell cholesterol. Developmental Cell 3(3): 306-8.

[30] Edwards, P.A., Tabor, D., Kast, H.R., 2000. Regulation of gene expression by SREBP and SCAP 1529: 103-13.

[31] Rome, S., Lecomte, V., Meugnier, E., Rieusset, J., Debard, C., Euthine, V., et al., 2008. Microarray analyses of SREBP-1a and SREBP-1c target genes identify new regulatory pathways in muscle. Physiological Genomics 34(3): 327-37.

[32] Hannah, V.C., Ou, J., Luong, A., Goldstein, J.L., Brown, M.S., 2001. Unsaturated Fatty Acids Down-regulate SREBP Isoforms 1a and 1c by Two Mechanisms in HEK293 Cells. Journal of Biological Chemistry 276(6): 4365-72.

[33] Mater, M.K., Thelen, A.P., Pan, D.A., Jump, D.B., 1999. Sterol response elementbinding protein 1c (SREBP1c) is involved in the polyunsaturated fatty acid suppression of hepatic S14 gene transcription. Journal of Biological Chemistry 274(46): 32725-32.

[34] Georgiadi, A., Kersten, S., 2012. Mechanisms of Gene Regulation by Fatty Acids. Advances in Nutrition 3(2): 127-34.

[35] Xu, J., Teran-Garcia, M., Park, J.H.Y., Nakamura, M.T., Clarke, S.D., 2001. Polyunsaturated Fatty Acids Suppress Hepatic Sterol Regulatory Element-binding Protein-1 Expression by Accelerating Transcript Decay. Journal of Biological Chemistry 276(13): 9800-7.

[36] Lee, J.N., Kim, H., Yao, H., Chen, Y., Weng, K., Ye, J., 2010. Identification of Ubxd8 protein as a sensor for unsaturated fatty acids and regulator of triglyceride synthesis. Proceedings of the National Academy of Sciences of the United States of America 107(50): 21424-9.

[37] Kim, H., Zhang, H., Meng, D., Russell, G., Lee, J.N., Ye, J., 2013. UAS domain of Ubxd8 and FAF1 polymerizes upon interaction with long-chain unsaturated fatty acids. Journal of Lipid Research 54(8): 2144-52.

[38] Ou, J., Tu, H., Shan, B., Luk, A., DeBose-Boyd, R. A Bashmakov, Y., Goldstein, J.L., et al., 2001. Unsaturated fatty acids inhibit transcription of the sterol regulatory element-binding protein-1c (SREBP-1c) gene by antagonizing ligand-dependent activation of the LXR. Proceedings of the National Academy of Sciences of the United States of America 98(11): 6027-32.

[39] Ulven, S.M., Dalen, K.T., Gustafsson, J.Å., Nebb, H.I., 2005. LXR is crucial in lipid metabolism. Prostaglandins Leukotrienes and Essential Fatty Acids 73(1 SPEC. ISS.): 59-63.

[40] Folch, J., Lees, M., Sloane Stanley, G.H., 1957. A simple method for the isolation and purification of total lipides from animal tissues. The Journal of Biological Chemistry 226(1): 497-509.

[41] Thompson, R.H., Merola, G. V., 1993. A simplified alternative to the AOAC official 
method for cholesterol in multicomponent foods. J AOAC Int. 76(5): 1057-68.

[42] Sakai, J., Rawson, R.B., Espenshade, P.J., Cheng, D., Seegmiller, A.C., Goldstein, J.L., et al., 1998. Molecular identification of the sterol-regulated luminal protease that cleaves SREBPs and controls lipid composition of animal cells. Molecular Cell 2(4): 505-14.

[43] Nohturfft, A., Yabe, D., Goldstein, J.L., Brown, M.S., Espenshade, P.J., 2000. Regulated step in cholesterol feedback localized to budding of SCAP from ER membranes. Cell 102(3): 315-23.

[44] Herzog, K., Pras-Raves, M.L., Vervaart, M.A.T., Luyf, A.C.M., van Kampen, A.H.C., Wanders, R.J.A., et al., 2016. Lipidomic analysis of fibroblasts from Zellweger spectrum disorder patients identifies disease-specific phospholipid ratios. Journal of Lipid Research 57(8): 1447-54.

[45] Irizarry, R.A., Hobbs, B., Collin, F., Beazer-Barclay, Y.D., Antonellis, K.J., Scherf, U., et al., 2003. Exploration, normalization, and summaries of high density oligonucleotide array probe level data. Biostatistics 4(2): 249-64.

[46] Bolstad, B.M., Irizarry, R.A., Astrand, M., Speed, T.P., 2003. A comparison of normalization methods for high density oligonucleotide array data based on variance and bias. Bioinformatics 19(2): 185-93.

[47] Dai, M., Wang, P., Boyd, A.D., Kostov, G., Athey, B., Jones, E.G., et al., 2005. Evolving gene/transcript definitions significantly alter the interpretation of GeneChip data. Nucleic Acids Research 33(20): 1-9.

[48] Yoshikawa, T., Shimano, H., Yahagi, N., Ide, T., Amemiya-Kudo, M., Matsuzaka, T., et al., 2002. Polyunsaturated fatty acids suppress sterol regulatory elementbinding protein 1c promoter activity by inhibition of liver X receptor (LXR) binding to LXR response elements. Journal of Biological Chemistry 277(3): 1705-11.

[49] Motamed, M., Zhang, Y., Wang, M.L., Seemann, J., Kwon, H.J., Goldstein, J.L., et al., 2011. Identification of luminal loop 1 of scap protein as the sterol sensor that maintains cholesterol homeostasis. Journal of Biological Chemistry 286(20): 1800212.

[50] Gao, Y., Zhou, Y., Goldstein, J.L., Brown, M.S., Radhakrishnan, A., 2017. Cholesterol-induced conformational changes in the sterolsensing domain of the Scap protein suggest feedback mechanism to control cholesterol synthesis. Journal of Biological Chemistry 292(21): 8729-37.

[51] Sato, R., Inoue, J., Kawabe, Y., Kodama, T., Takano, T., Maeda, M., 1996. Steroldependent Transcriptional Regulation of Sterol Regulatory Element-binding Protein2. The Journal of Biological Chemistry 271(43): 26461-4.

[52] Amemiya-Kudo, M., Shimano, H., Yoshikawa, T., Yahagi, N., Hasty, A.H., Okazaki, H., et al., 2000. Promoter analysis of the mouse sterol regulatory element-binding protein-1c gene. Journal of Biological Chemistry 275(40): 31078-85.

[53] Matsuda, M., Korn, B.S., Hammer, R.E., Moon, Y.A., Komuro, R., Horton, J.D., et al., 2001. SREBP cleavage-activating protein (SCAP) is required for increased lipid 
synthesis in liver induced by cholesterol deprivation and insulin elevation. Genes and Development 15(10): 1206-16.

[54] Yang, T., Espenshade, P.J., Wright, M.E., Yabe, D., Gong, Y., Aebersold, R., et al., 2002. Crucial Step in Cholesterol Homeostasis: Sterols Promote Binding of SCAP to INSIG-1, a Membrane Protein that Facilitates Retention of SREBPs in ER. Cell 110: 489-500.

[55] Yabe, D., Brown, M.S., Goldstein, J.L., 2002. Insig-2, a second endoplasmic reticulum protein that binds SCAP and blocks export of sterol regulatory elementbinding proteins. Proceedings of the National Academy of Sciences 99(20): 127538 .

[56] Koppe, S.W.P., Elias, M., Moseley, R.H., Green, R.M., 2009. Trans fat feeding results in higher serum alanine aminotransferase and increased insulin resistance compared with a standard murine high-fat diet. Am J Physiol Gastrointest Liver Physiol 297(2): G378-84.

[57] Tetri, L.H., Basaranoglu, M., Brunt, E.M., Yerian, L.M., Neuschwander-Tetri, B.A., 2008. Severe NAFLD with hepatic necroinflammatory changes in mice fed trans fats and a high-fructose corn syrup equivalent. AJP: Gastrointestinal and Liver Physiology 295(5): G987-95.

[58] Morinaga, M., Kazuyoshi Kon, ${ }^{*}, \dagger$ Hiroaki Saito, Kumiko Arai, Hiromi Kusama, A.U., Shunhei Yamashina, K.I. and S.W., 2015. Sodium 4-phenylbutyrate prevents murine dietary steatohepatitis caused by trans-fatty acid plus fructose. Journal of Clinical Biochemistry and Nutrition 56(3): 166-70.

[59] den Hartigh, L.J., Han, C.Y., Wang, S., Omer, M., Chait, A., 2013. 10E,12Zconjugated linoleic acid impairs adipocyte triglyceride storage by enhancing fatty acid oxidation, lipolysis, and mitochondrial reactive oxygen species. Journal of Lipid Research 54(11): 2964-78.

[60] Clément, L., Poirier, H., Niot, I., Bocher, V., Guerre-Millo, M., Krief, S., et al., 2002. Dietary trans-10,cis-12 conjugated linoleic acid induces hyperinsulinemia and fatty liver in the mouse. Journal of Lipid Research 43(9): 1400-9.

[61] Ioannou, G.N., 2016. The Role of Cholesterol in the Pathogenesis of NASH. Trends in Endocrinology and Metabolism 27(2): 84-95.

[62] Wouters, K., Bilsen, M. van., Gorp, P.J. va., Bieghs, V., Lütjohann, D., Kerksiek, A., et al., 2010. Intrahepatic cholesterol influences progression, inhibition and reversal of non-alcoholic steatohepatitis in hyperlipidemic mice. FEBS Letters 584(5): 10015 .

[63] Zock, P.L., Katan, M.B., 1992. Hydrogenation alternatives: effects of trans fatty acids and stearic acid versus linoleic acid on serum lipids and lipoproteins in humans. Journal of Lipid Research 33(3): 399-410.

[64] Judd, J.T., Clevidence, B.A., Muesing, R.A., Wittes, J., Sunkin, M.E., Podczasy, J.J., 1994. Dietary trans fatty acids: Effects on plasma lipids and lipoproteins of healthy men and women. American Journal of Clinical Nutrition. 


\section{Supplemental Material}

A

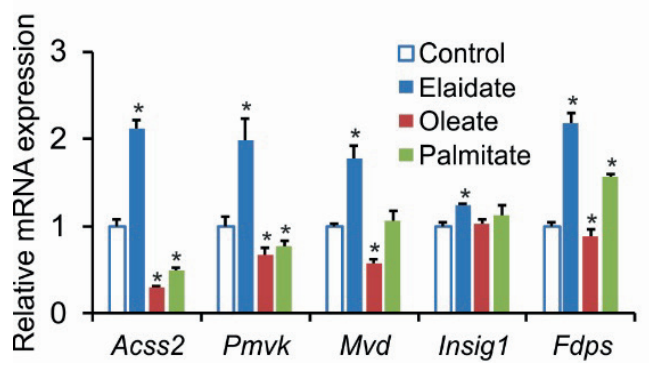

B

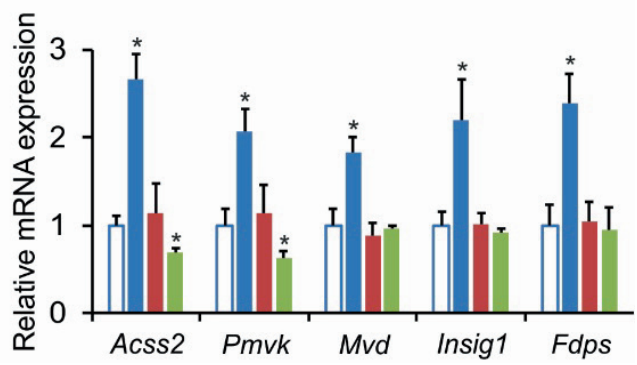

Supplemental Figure 1: Elaidate induces cholesterogenic genes in 3T3-L1 cells. (A) Relative mRNA expression of cholesterol synthesis genes in undifferentiated 3T3-L1 fibroblasts treated with $500 \mu \mathrm{M}$ of individual fatty acids for 24 hours. (B) Relative mRNA expression of cholesterol synthesis genes in 3T3-L1 cells that were differentiated in the presence of $500 \mu \mathrm{M}$ of individual fatty acids. mRNA expression was normalized to $36 b 4$. Data are mean \pm SD. ${ }^{*} p<0.05$ relative to control. 


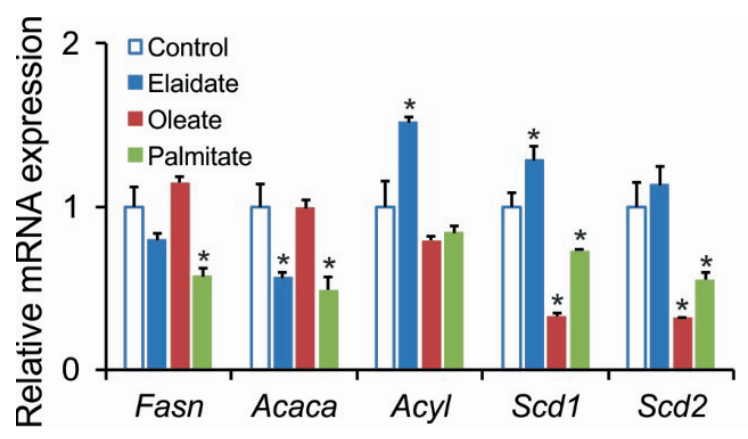

Supplemental Figure 2: Relative mRNA expression of target genes of SREBP1c involved in fatty acid synthesis in Hepa1-6 cells treated with $500 \mu \mathrm{M}$ of individual fatty acids for 24 hours. mRNA expression was normalized to $36 b 4$. Data are mean \pm SD. ${ }^{*} \mathrm{p}<0.05$ relative to control condition. 
A

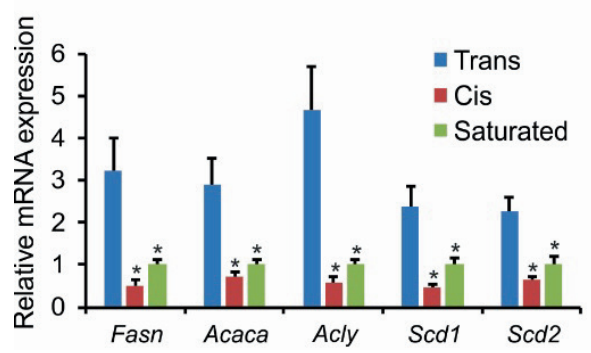

B

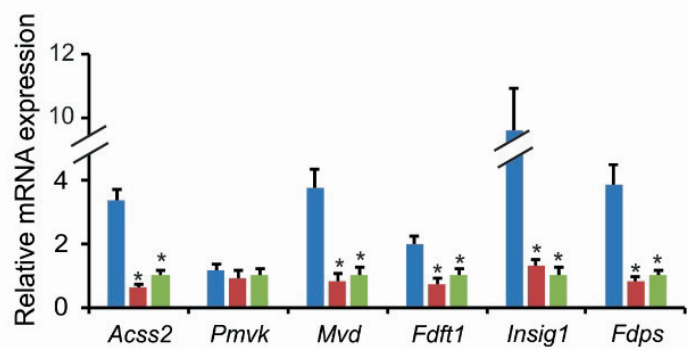

Supplemental Figure 3: Trans diet induces lipogenic and cholesterogenic genes in inguinal adipose tissue. (A-B) Relative mRNA expression of fatty acid synthesis genes (A) and cholesterol synthesis genes (B) in the inguinal adipose tissue of mice fed Trans, Cis and Saturated diets. mRNA expression was normalized to $36 b 4$. $\mathrm{N}=8$ mice/group. Data are mean \pm SEM. $* \mathrm{p}<0.05$ relative to Trans group. 


\section{Supplemental Table 1: Composition of test diets}

\begin{tabular}{|c|c|c|c|}
\hline Ingredients (\%) & Saturated fat diet & Unsaturated fat diet & Trans fat diet \\
\hline Cocoa Butter & 23.3060 & 0 & 0 \\
\hline Canola Oil & 0 & 13.2265 & 0 \\
\hline Palm Olein Oil & 0 & 7.8536 & 0 \\
\hline Hydrogenated Soy Oil & 0 & 0 & 21.0800 \\
\hline Casein- Vitamin Tested & 23.3060 & 23.3059 & 23.3060 \\
\hline Sucrose & 19.7090 & 19.7090 & 19.7090 \\
\hline Maltodextrin & 11.6530 & 11.6530 & 11.6530 \\
\hline Dextrin & 8.4830 & 8.4830 & 8.4830 \\
\hline Powdered Cellulose & 5.8270 & 5.8270 & 5.8270 \\
\hline Soybean Oil & 2.9200 & 2.9200 & 2.9200 \\
\hline Potassium Citrate, & 1.9230 & 1.9230 & 1.9230 \\
\hline \multicolumn{4}{|l|}{ Tribasic Monohydrate } \\
\hline Calcium Phosphate & 1.5150 & 1.5150 & 1.5150 \\
\hline AIN-76A Vitamin Mix & 1.1650 & 1.1650 & 1.1650 \\
\hline DIO Mineral Mix & 1.1650 & 1.1650 & 1.1650 \\
\hline Calcium Carbonate & 0.6410 & 0.6410 & 0.6410 \\
\hline L-Cystine & 0.3500 & 0.3500 & 0.3500 \\
\hline Choline Bitartate & 0.2330 & 0.2330 & 0.2330 \\
\hline FD\&C Yellow 6 Lake & 0.0300 & 0.0300 & 0.0300 \\
\hline
\end{tabular}




\section{Supplemental Table 2: Primer sequences of genes}

\begin{tabular}{|c|c|c|}
\hline Genes & Forward primer & Reverse primer \\
\hline m36B4 & ATGGGTACAAGCGCGTCCTG & GCCTTGACCTTTTCAGTAAG \\
\hline mFasn & GGCATCATTGGGCACTCCTT & GCTGCAAGCACAGCCTCTCT \\
\hline mAcyl & CCAGTTAATCAAACGTCGAGGA & CTTTGGCCTTGCCGACAGT \\
\hline mScd1 & TAGCCTGTAAAAGATTTCTGCAAACC & CCGGAGACCCTTAGATCGA \\
\hline $\mathrm{mScd} 2$ & TTCTCCCGAGAGCTAATGTTCT & TTCTTGCGATACGCCGTGG \\
\hline mAcss 2 & AAACACGCTCAGGGAAAATCA & ACCGTAGATGTATCCCCCAGG \\
\hline mPmvk & AAAATCCGGGAAGGACTTCGT & AGAGCACAGATGTTACCTCCA \\
\hline mMvd & ATGGCCTCAGAAAAGCCTCAG & TGGTCGTTTTTAGCTGGTCCT \\
\hline mFdft1 & TCCCACTGCTGTGTAACTTCC & TGTCTACAAATTCTGCCATCCC \\
\hline mInsig1 & TGTCGGTTTACTGTATCCCTGT & GGCAAAATCTAATTTGGCACTGG \\
\hline mFdps & GGAGGTCCTAGAGTACAATGCC & AAGCCTGGAGCAGTTCTACAC \\
\hline mSrebpla & GCCAGCCTGGCAATGTGTGA & CAGGTCACACAGGAGCAGCT \\
\hline mSrebp1c & c GGAGCCATGGATTGCACATT & CCTGTCTCACCCCCAGCATA \\
\hline mSrebp2 & CTGCAGCCTCAAGTGCAAAG & CAGTGTGCCATTGGCTGTCT \\
\hline mHmgcr & AGCTTGCCCGAATTGTATGTG & TCTGTTGTGAACCATGTGACTTC \\
\hline mLdlr & GCATCAGCTTGGACAAGGTGT & GGGAACAGCCACCATTGTTG \\
\hline mScap & TGGAGCTTTTGAGACTCAGGA & TCGATTAAGCAGGTGAGGTCG \\
\hline mUbxd8 & GAGCAGGATCTAACTCAGGAGC & CAGCAGCCTCCATGTTCCAG \\
\hline mLxra & CTCAATGCCTGATGTTTCTCCT & TCCAACCCTATCCCTAAAGCAA \\
\hline mLxrb & CGTGGTCATCTTAGAGCCAGA & GCTGAGCACGTTGTAGTGGAA \\
\hline
\end{tabular}




\section{Supplemental Table 2 continued: Primer sequences of genes}

\begin{tabular}{|c|c|c|}
\hline Genes & Forward primer & Reverse primer \\
\hline mAbcal & AAAACCGCAGACATCCTTCAG & CATACCGAAACTCGTTCACCC \\
\hline mAbcg1 & СТTTССТАСТСТGTACCCGAGG & CGGGGCATTCCATTGATAAGG \\
\hline mSoat1 & GAAGGCTCACTCATTTGTCAGA & GTCTCGGTAAATAAGTGTAGGCG \\
\hline mSqle & ATAAGAAATGCGGGGATGTCAC & ATATCCGAGAAGGCAGCGAAC \\
\hline hGAPDH & GAAGGTGAAGGTCGGAGTC & GAAGATGGTGATGGGATTTC \\
\hline hSREBP1 & ACTTCCCTGGCCTATTTGACC & GGCATGGACGGGTAAATCTT \\
\hline hSREBP2 & AACGGTCATTCACCCAGGTC & GGCTGAAGAATAGGAGTTGCC \\
\hline hACSS2 & CAAGTGTGTCAGTTCAGCAATG & CCACAAGCTCTGGGATCATAGG \\
\hline hHMGCR & GGACCCCTTTGCTTAGATGAAA & CCACCAAGACCTATTGCTCTG \\
\hline hHMGCS1 & CTCTTGGGATGGACGGTATGC & GCTCCAACTCCACCTGTAGG \\
\hline hMVD & GGACCGGATTTGGCTGAATG & CCCATCCCGTGAGTTCCTC \\
\hline hINSIG1 & CCTGGCATCATCGCCTGTT & AGAGTGACATTCCTCTGGATCTG \\
\hline hSQLE & TGACAATTCTCATCTGAGGTCCA & CAGGGATACCCTTTAGCAGTTTT \\
\hline hLDLR & GACGTGGCGTGAACATCTG & CTGGCAGGCAATGCTTTGG \\
\hline
\end{tabular}




\section{Supplemental Table 3: Detailed fatty acid profile of diets}

Fatty acids $\quad$ Saturated (\%) $\quad$ Cis-Unsaturated (\%) $\quad$ Trans-Unsaturated (\%)

\begin{tabular}{|c|c|c|c|}
\hline C $14: 0$ & 0.18 & 0.44 & 0.14 \\
\hline C $14: 19 t$ & 0 & 0 & 0 \\
\hline C $14: 19 c$ & 0 & 0 & 0 \\
\hline C 16:0 & 23.76 & 16.16 & 11.01 \\
\hline C $16: 19 t$ & 0 & 0 & 0 \\
\hline C $16: 19 \mathrm{c}$ & 0.18 & 0.26 & 0 \\
\hline C 17:0 & 0.28 & 0 & 0.17 \\
\hline C $17: 110 \mathrm{c}$ & 0 & 0 & 0 \\
\hline C 18:0 & 31.59 & 2.99 & 8.23 \\
\hline C $18: 17+8 t$ & 0.11 & 0 & 4.28 \\
\hline C $18: 19 t$ & 0 & 0.11 & 3.33 \\
\hline C $18: 111 \mathrm{t}$ & 0 & 0 & 6.66 \\
\hline C $18: 15 c+7 c+12 t$ & 0 & 0 & 5.03 \\
\hline C $18: 19 c+13 t$ & 30.98 & 49.24 & 23.84 \\
\hline C $18: 111 c+15 t$ & 0.87 & 2.54 & 2.05 \\
\hline C $18: 112 c$ & 0 & 0.23 & 6.85 \\
\hline C $18: 113 \mathrm{c}$ & 0 & 0.10 & 0.91 \\
\hline C $18: 115 c$ & 0 & 0 & 0.65 \\
\hline C $18: 2 \mathrm{tt}$ & 0 & 0 & 0.78 \\
\hline C $18: 2 \mathrm{ct}$ & 0 & 0.19 & 0 \\
\hline C $18: 2$ tc & 0 & 0.11 & 0 \\
\hline C 18:2 cc & 9.67 & 19.96 & 7.56 \\
\hline C $18: 3 \mathrm{ttt}$ & 0 & 0 & 0 \\
\hline C $20: 0$ & 1.10 & 0.55 & 0.31 \\
\hline C 18:3 tct & 0 & 0 & 0 \\
\hline C $18: 3 \mathrm{ctt}$ & 0 & 0 & 0 \\
\hline C $18: 3 \mathrm{ctc}$ & 0 & 0.09 & 0 \\
\hline C $18: 3$ tcc & 0 & 0.19 & 0 \\
\hline C $18: 3 \mathrm{ccc}$ & 1.16 & 5.03 & 1.00 \\
\hline C $20: 111 \mathrm{c}$ & 0 & 0.84 & 0.21 \\
\hline Unspecified cis/trans & 0 & 0 & 11.01 \\
\hline Unknown fatty acids & 0.14 & 1.04 & 6.03 \\
\hline
\end{tabular}


Chapter 4 


\section{Feeding Angptl4/-- mice trans fat promotes foam cell formation in}

mesenteric lymph nodes without leading to ascites

Antwi-Boasiako Oteng, Asmita Bhattacharya, Susanne Brodesser, Ling Qi, Nguan Soon Tan, Sander Kersten

Journal of Lipid Research 2017 58(6):1100-1113 


\section{Abstract}

Background: ANGPTL4 regulates plasma triglyceride levels by inhibiting lipoprotein lipase. Inactivation of ANGPTL4 decreases plasma triglycerides and reduces risk of coronary artery disease. Unfortunately, targeting ANGPTL4 for the therapeutic management of dyslipidemia and atherosclerosis is hampered by the observation that mice and monkeys in which ANGPTL4 is inactivated exhibit lipid accumulation in mesenteric lymph nodes. In mice these pathological events exclusively unfold upon feeding a high saturated fatty acid diet and are followed by an ultimately lethal proinflammatory response and chylous ascites.

Results: Here we show that Angptl4-/- mice fed a diet rich in trans fatty acids develop numerous lipid-filled giant cells in their mesenteric lymph nodes, yet do not have elevated serum amyloid and haptoglobin, do not exhibit ascites, and survive, unlike Angptl4-/- mice fed a saturated fatty acid-rich diet. In RAW264.7 macrophages the saturated fatty acid palmitate markedly increases markers of inflammation and the unfolded protein response, whereas the trans-unsaturated elaidate and the cis-unsaturated oleate have the opposite effect.

Conclusion: Trans and saturated fatty acids have very distinct biological effects. Furthermore, lipid accumulation in mesenteric lymph nodes is uncoupled from activation of an acute-phase response and chylous ascites, suggesting that ANGPTL4 should not be fully dismissed as target for dyslipidemia. 


\section{Introduction}

Elevated plasma levels of LDL-cholesterol are widely accepted to accelerate the development of atherosclerosis. Besides LDL, elevated plasma triglycerides are increasingly recognized as a risk factor for coronary artery disease. More specifically, recent human genetic studies have pointed towards a causal role of hypertriglyceridemia in atherosclerosis development. Accordingly, strategies aimed at reducing hypertriglyceridemia in humans offer a promising therapeutic approach to lower the risk of coronary artery disease. Triglycerides are present in the blood as part of chylomicrons and very low density lipoproteins, and are hydrolysed by the enzyme lipoprotein lipase (LPL) [1]. Hence, enhanced hydrolysis of triglycerides by LPL attenuates hypertriglyceridemia and may be targeted to retard the development of coronary artery disease [2].

LPL is produced by a number of cell types including (cardio)myocytes, adipocytes, and macrophages, and is transported to the luminal surface of capillaries by the protein glycosylphosphatidylinositol-anchored HDL binding protein 1 (GPIHBP1), which also helps to stabilize LPL [3]. Consequently, inactivating mutations in GPIHBP1 give rise to hyperchylomicronemia in mice and humans $[4,5]$. The activity of LPL is under tight regulation by several proteins to ensure the homeostatic balance in triglyceride disposal under different nutritional and physiological states [6,7]. One of these proteins is Angiopoietin-like 4 (ANGPTL4). Following its discovery in 2000 [8,9], ANGPTL4 has been shown to post-translationally inhibit LPL activity by promoting the conversion of catalytically active LPL dimers into inactive monomers and by stimulating the intracellular degradation of LPL [10,11]. As a result, over-expression of ANGPTL4 in mice leads to increased plasma triglycerides, whereas the deletion of ANGPTL4 results in lower plasma triglycerides [12,13]. Interestingly, the loss of ANGPTL4 is able to correct the hypertriglyceridemia in Gpihbp 1-/- mice [14], suggesting that ANGPTL4 may interfere with an accessory transport mechanism for LPL across the endothelium [3]. Alternatively, the pronounced lipid lowering caused by loss of ANGPTL4 in Gpihbp1-/- mice may be due to enhanced entry of LPL into the bloodstream via the lymph [10].

In humans, carriers of the inactivating E40K mutation in ANGPTL4 have lower plasma triglyceride levels and have a reduced risk of coronary artery disease compared to noncarriers $[15,16]$. Based on these observations, ANGPTL4 would seem a very attractive pharmacological target to decrease the risk of developing coronary artery disease. However, we and others have shown that inactivating ANGPTL4 in mice fed a diet high 
in saturated fat elicits severe and ultimately lethal chylous ascites and peritonitis, which is preceded by enlarged mesenteric lymph nodes (MLN), the presence of lipid-filled giant cells, and a massive acute phase response [17]. These effects were coupled to the role of ANGPTL4 as LPL inhibitor in MLN macrophages, thereby preventing excessive fatty acid uptake and a concomitant pro-inflammatory response. The above data have raised serious concerns about the safety of pharmacological targeting of ANGPTL4 in patients. Recently, Dewey et al. reported the accumulation of lipids in MLN of several female monkeys treated with an anti-ANGPTL4 antibody [16]. However, it is presently unclear whether the accumulation of lipid in MLN forebodes a marked acute phase response, and is directly connected to the development of chylous ascites and peritonitis. In this context, it is relevant to mention that Dewey et al. did not observe any significant difference between E40K carriers and non-carriers in the rates of diagnosis codes for abdominal lymphatic disorders, as well as ascites, peritonitis, malabsorption, abdominal discomfort, or diarrhea [16].

Trans fatty acids are unsaturated fatty acids that contain one or more double bonds in the trans configuration as opposed to the more common cis-configuration. Trans fatty acids are synthesized naturally in small amounts in the guts of certain ruminants and are thus contained in meat and dairy products of these animals $[18,19]$. However, most of the ingested trans fatty acids are synthesized artificially during food processing by partial hydrogenation of vegetable oils to improve the texture and longevity of foods. Numerous epidemiological studies have demonstrated a strong correlation between consumption of industrially-produced trans fatty acids and the development of cardiovascular diseases [20-24]. Consequently, national policies that either restrict or ban the consumption of trans fat-rich foods have been instituted by several countries [25-29].

A trans double bond preserves the straight conformation of a fatty acid, whereas a regular cis double bond creates a kink in the fatty acid molecule. Because they have a similar linear structure, trans fatty acids are claimed to act like saturated fatty acids. Indeed, both trans and saturated fatty acids have been shown to raise plasma levels of LDL, which may account for the positive link between trans and saturated fatty acid consumption and coronary artery disease [22]. However, to what extent trans and saturated fatty acids are interchangeable and share the same cellular effects in vivo and in vitro is not well known. To further explore this question, we compared the effect of diets rich in trans or saturated fatty acids in Angptl4-/- mice, which as pointed out above develop a massive acute phase response and ultimately lethal chylous ascites upon feeding a saturated fat-rich diet. These studies were complemented by investigations into the effect of saturated and trans fatty acids in cultured macrophages. Our results indicate that: a) trans fatty acids have 
very distinct effects from saturated fatty acids in vitro and in vivo; b) the accumulation of lipid in MLN is uncoupled from the hepatic acute phase response and chylous ascites in Angptl4-/- mice fed a diet rich in trans fatty acids. 


\section{Materials and Methods}

\section{Animal Treatment}

Animal studies were conducted using pure-bred Angptl4 F $^{-}$mice on a $\mathrm{C} 57 \mathrm{Bl} / 6$ background. Four- to six-months-old male mice were randomly assigned to 3 groups ( $n=8$ per group). Each group was fed a high fat diet rich in saturated, cis-unsaturated or trans-unsaturated fatty acids for 7 weeks providing 45\% energy percent as triglycerides (Modified Testdiet 58V8, TestDiet), shown in Supplemental Table 1 (Test Diet Limited, London). Fat sources were partially hydrogenated soy oil for the trans fat diet, canola and palm olein oil for the unsaturated fat diet, and cocoa butter for the saturated fat diet. Bodyweight and food intake were measured weekly. Blood samples were collected at weekly intervals by tail vein bleeding into EDTA-coated tubes. After the dietary intervention, mice were anaesthetized with isoflurane, followed by blood collection via orbital puncture. Mice were euthanized by cervical dislocation, after which tissues were excised. Tissues for RNA and protein analysis were immediately frozen in liquid nitrogen and stored at $-80^{\circ} \mathrm{C}$. Tissues for histological analysis were fixed in $4 \%$ paraformaldehyde and later embedded in paraffin. In a second study, 9 male Angptl4 ${ }^{-}$ and 8 male wildtype mice at 4-6 months of age were placed on the trans-fat diet for 7 weeks purposely to confirm and to quantify foam cell formation in the MLN. These animal studies were approved by the Local Animal Ethics Committee at Wageningen University.

\section{Fatty acid profiling of test diets by Gas Chromatography - Flame Ionization Detection}

The fat component in the test diets was extracted by the Folch technique as previously described [30]. Briefly, the fats were extracted with a mixture of chloroform and ethanol (2:1 ratio). The addition of water gave a biphasic system, with the fat contained in the chloroform at lower phase. Following purification, the extracted fats were measured gravimetrically. The isolated fats were then saponificated with methanolic $\mathrm{NaOH}$ and methylated with boron trifluoride $\left(\mathrm{BF}_{3}\right)$ to obtain methyl esters. The methyl esters were then fractionated by gas chromatography, detected by flame ionization and calculated as a fraction of the total amount.

\section{Histology}

Hematoxylin and eosin (H\&E) staining of MLN was performed using standard protocols. In summary, the MLN were fixed in 4\% paraformaldehyde, processed and embedded in 
paraffin blocks. The tissues were then sectioned onto a superfrost glass slide at $3 \mu \mathrm{m}$ with a microtome. The tissue slices were incubated overnight at $37^{\circ} \mathrm{C}$. The slices were stained at room temperature in Mayer hematoxylin solution for $10 \mathrm{~min}$ and in eosin $\mathrm{Y}$ solution for $10 \mathrm{sec}$. Images were then taken with a light microscope.

\section{Quantification of plasma acute phase proteins, insulin, and glucose.}

Blood samples were centrifuged at $4^{\circ} \mathrm{C}$ for $15 \mathrm{~min}$ at $10,000 \mathrm{~g}$. Plasma was collected and stored at $-80^{\circ} \mathrm{C}$. ELISA Development kits were used to measure plasma serum amyloid A (Tridelta Dev. Ltd, Ireland), Haptoglobin (Abcam, Cambridge-UK) and Lipocalin-2 (Abcam, Cambridge-UK) according to the manufacturer's protocol. Plasma insulin and glucose were measured on plasma samples collected after 2 weeks of dietary intervention, prior to the mice getting visibly ill. Glucose (DiaSys, Germany) and insulin (Crystal Chem, Downers Grove, USA) were measured using dedicated kits.

\section{RNA isolation and quantitative real-time PCR}

Total RNA was isolated from mouse liver tissue or cultured RAW264.7 macrophages with TRIzol reagent (Invitrogen, Bleiswijk, the Netherlands) and a Tissuelyser II (Qiagen, Venlo, the Netherlands). Reverse transcription was performed using First strand cDNA synthesis kit (Thermo Scientific, IL, USA) according to the manufacturer's protocol. Quantitative RT-PCR-amplifications were performed with iQ SYBR Green Supermix on a CFX384 Touch real-time PCR detection system (Bio-Rad, Hercules, CA, USA). Primer sequences of genes are provided in Supplemental Table 2. Expression of target genes were normalised to $36 \mathrm{~b} 4$.

\section{Cell culture}

Murine RAW264.7 macrophages (ATCC) were cultured in Dulbecco's modified Eagle's medium (DMEM) supplemented with $10 \%$ fetal bovine serum, $100 \mathrm{U} / \mathrm{ml}$ penicillin, and $1000 \mu \mathrm{g} / \mathrm{ml}$ streptomycin (Lonza, Verviers, Belgium) in a humidified chamber at $37{ }^{\circ} \mathrm{C}$ with $5 \% \mathrm{CO}_{2}$.

\section{Fatty acid preparation and treatment}

Palmitate, oleate and elaidate (Sigma-Aldrich, St Louis, MO, USA) served as models of saturated, cis-unsaturated and trans-unsaturated fatty acids respectively. The fatty acids were reconstituted in ethanol and prepared by dissolving in filter-sterilised $70 \mathrm{mM} \mathrm{KOH}$. The fatty acids were coupled to FA-free BSA by 30 min incubation at $37^{\circ} \mathrm{C}$ in a prewarmed DMEM containing FA-free BSA. The final working concentrations of the FAs was $500 \mu \mathrm{M}$ and the final ratio of FFA:BSA was $2: 1$. Vehicle control treatment was 
prepared using an equivalent mixture of $\mathrm{KOH}$ and ethanol. The final concentration of ethanol was less than $0.1 \%$. The RAW264.7 cells were seeded into 6 -well plates at $4 \mathrm{x}$ $10^{5}$ cells per well. After overnight incubation, the cells were incubated with $500 \mu \mathrm{M}$ of the individual fatty acids or vehicle control for 6 hours. Treatments for experiments were performed in triplicates unless specified otherwise. This procedure was repeated in subsequent experiments with different trans fatty acids (elaidate, trans-vaccenate, palmitelaidate, CLA 9Z,11E and CLA 10E, 12Z) and different saturated fatty acids (laurate, myristate, palmitate and stearate).

\section{Fatty acid treatment of RAW264.7 cells in the presence of chemical inhibitors}

Palmitate-treated RAW264.7 cells were co-treated with several chemical inhibitors. Specifically, RAW264.7 cells were treated with $500 \mu \mathrm{M}$ palmitate for 6 hours, and cotreated for the same duration with $10 \mu \mathrm{M}$ dimethylsphingosine (DMS) (a sphingosine kinase inhibitor), $100 \mu \mathrm{M}$ sulfosuccinimidyl olate (SSO) (CD36 inhibitor), $2.5 \mu \mathrm{M}$ Triascin C (Acyl-CoA synthase inhibitor), $50 \mu \mathrm{M} \mathrm{C}$-ceramide (membrane-permeable ceramide analog), $10 \mu \mathrm{M}$ fumonisin B1 (ceramide synthase inhibitor), $10 \mu \mathrm{M}$ amidepsine A (Diglyceride acyltransferase 1 (DGAT1) inhibitor), $50 \mu \mathrm{M}$ etomoxir (carnitine palmitoyl transferase 1 (Cpt1) inhibitor) or $10 \mu \mathrm{M}$ myriocin (serine palmitoyltransferase 1 (SPT1) inhibitor). In a follow-up experiment, RAW 264.7 cells were treated with $500 \mu \mathrm{M}$ palmitate, oleate or elaidate for 6 hours, and co-treated with $2.5 \mu \mathrm{M}$ Triascin $\mathrm{C}$ or $10 \mu \mathrm{M}$ DMS.

\section{Oil red-O staining of lipid droplets}

A stock of oil red-O was prepared by dissolving $0.5 \mathrm{~g}$ in $500 \mathrm{~mL}$ of isopropanol. A working concentration was prepared by mixing the stock and $\mathrm{ddH}_{2} \mathrm{O}$ at a ratio of $3: 2$. After treatment, the RAW264.7 cells were washed twice with PBS, fixed with 4\% paraformaldehyde for $30 \mathrm{~min}$ and incubated with filtered oil red-O dye for $20 \mathrm{mins}$. The cells were then carefully washed three times with $\mathrm{ddH}_{2} \mathrm{O}$. Pictures were taken by light microscope.

\section{Triglyceride quantification}

Following treatment, cells were washed twice with PBS. Tris-EDTA buffer (25 mM Tris, $1 \mathrm{mM}$ EDTA; pH7.5) was added to the cells and to prepared triglyceride standards (Instruchemie, The Netherlands). The plate was subsequently put at $-80^{\circ} \mathrm{C}$ for 1 hour. The plates were allowed to thaw to room temperature, followed by the addition of 200 $\mu \mathrm{L}$ tertiary butanol and $50 \mu \mathrm{L}$ methanol. The plates were then put to shake at $270 \mathrm{rpm}$ for 15 mins and allowed to evaporate on a hot plate at $60^{\circ} \mathrm{C}$. Monocolor reagent 
(Instruchemie, The Netherlands) were then added to the wells, followed by shaking for 5 mins at $250 \mathrm{rpm}$ on a plate shaker. Samples and standards were transferred to 96-well plates and absorbance was measured at $492 \mathrm{~nm}$. Triglyceride content was normalized to protein content, which was quantified by the Pierce BCA protein assay (ThermoScientific, IL, USA) according to manufacturer's protocol.

\section{Measurement of Oxidative stress}

Intracellular ROS production was measured using DCFDA Cellular ROS Detection Assay kit (Abcam, ab11385, Cambridge, UK) according to manufacturer's protocol. In brief, RAW264.7 cells were seeded into 96-well plates at $2 \times 10^{4}$ cells per well. After overnight incubation, cells were washed with PBS and incubated with $20 \mu \mathrm{M}$ DCFDA dye for 45 mins at $37^{\circ} \mathrm{C}$ in the dark. The cells were then washed with PBS and incubated for 6 hours with fatty acids or vehicle control. DCFDA oxidation by intracellular ROS generates a fluorescent dicholorofluorescein (DCF). Fluorescence signal was measured at excitation/emission wavelengths Ex485/Em538 nm with a Fluoroskan Ascent FL microplate reader. Six replicates were used per individual fatty acid and control treatment.

\section{Immunoblot of UPR markers}

Cell lysates were prepared with RIPA lysis buffer supplemented with protease and phosphatase inhibitors. Protein concentrations in lysates was quantified via Bradford assay (Biorad, CA, USA). Samples were boiled for $5 \mathrm{~min}$ in SDS-containing buffer before 30ug of protein lysates were loaded in a mini SDS-PAGE gel. Phos-tag gels were run similar to regular SDS-PAGE gels complemented with $50 \mu \mathrm{M} \mathrm{MnCl2} \mathrm{(Sigma)} \mathrm{and}$ $50 \mu \mathrm{M}$ Phos-tag (NARD), and by soaking gels for $10 \mathrm{mins}$ in $1 \mathrm{mM}$ EDTA before transfer to PVDF membrane as previously described [31]. The primary antibodies which were diluted in 2\% BSA in TBST are as follows: HSP90 (\#7947, 1:5000), BiP (\#1051, 1:500), $\alpha$-Tubulin (\#5286, 1:2000) from Santa Cruz; IRE1 $\alpha$ (\#3294, 1:2000), PERK (\#3192, 1:2000), cleaved caspase-3 (\#9664, 1:1000) from Cell Signaling; Sel1L (ab78298, 1:2,000), CHOP (ab11419, 1:1000) from Abcam; Xbp1 (\#9D11A43, 1:1000) from BioLegend; Hrd1 (kind gift from Dr. Richard Wojcikiewicz 1:200). The secondary antibodies which were diluted in 5\% milk in TBST are anti-rabbit IgG-HRP (Biorad, 1:5000), anti-mouse IgG-HRP (Biorad, 1:5000); and anti-goat IgG-HRP (Jackson ImmunoResearch, 1:5000). 


\section{Microarray analysis}

Microarray analysis was performed on RAW264.7 macrophages treated with either palmitate, oleate or elaidate $(500 \mu \mathrm{M})$ for 6 hours. RNA was purified with RNeasy Minikit columns (Qiagen) and analyzed for quality with RNA 6000 Nano chips on the Agilent 2100 Bioanalyzer (Agilent Technologies, Amsterdam, The Netherlands). One microgram of RNA was used for cDNA synthesis using the First Strand cDNA synthesis kit (Thermo Scientific IL, USA). Purified RNA (100 ng) was labeled with the Ambion WT expression kit (Invitrogen) and hybridized to an Affymetrix Mouse Gene 1.1 ST array plate (Affymetrix, Santa Clara, CA). Hybridization, washing, and scanning were carried out on an Affymetrix GeneTitan platform. Scans of the Affymetrix arrays were processed using packages from the Bioconductor project. Arrays were normalized using the Robust Multi-array Average method [32,33]. Probesets were defined by assigning probes to unique gene identifiers, e.g. Entrez ID [34]. The total gene set (22135 probesets) was filtered according to the Universal Probability Code method (score $>0.5$ ) [35], resulting in 7905 probesets, which were used to generate a scatter plot. An overall heatmap was generated using the 500 most variable genes based on calculation of the coefficient of variation. UPC-filtered genes that were induced at least 1.5 fold by palmitate or elaidate relative to oleate were used for Ingenuity Pathway Analysis (Ingenuity Systems, Redwood City, USA) Microarray data were submitted to the Gene Expression Omnibus (accession number SE98303).

\section{Lipid analysis}

Ceramide and sphingomyelin levels were determined by Liquid Chromatography coupled to Electrospray Ionization Tandem Mass Spectrometry (LC-ESI-MS/MS). RAW 264.7 macrophages from $10 \mathrm{~cm}$ cell culture dishes were homogenized in $400 \mu \mathrm{l}$ of water using the Precellys 24 Homogenisator (Peqlab, Erlangen, Germany) at 6,500 rpm for $30 \mathrm{sec}$. To $100 \mu \mathrm{l}$ of homogenate $500 \mu \mathrm{l}$ of methanol, $250 \mu \mathrm{l}$ of chloroform and internal standards (127 pmol Ceramide 12:0 and 123 pmol Sphingomyelin 12:0, both Avanti Polar Lipids, Alabaster, AL, USA) were added. Lipid extraction was performed as previously described [36]. Dried lipid extracts were resolved in $300 \mu 1$ of mobile phase solvent A (see below). LC-MS/MS analysis was performed using a normal phase Nucleosil NH2 column $(50 \mathrm{~mm} \times 2 \mathrm{~mm}$ ID, $3 \mu \mathrm{m}$ particle size, $120 \AA$ pore size, Macherey-Nagel, Dueren, Germany) with detection using a QTRAP 6500 mass spectrometer (SCIEX, Darmstadt, Germany). The LC (1260 Infinity Binary LC System, Agilent, Waldbronn, Germany) was operated at a flow rate of $0.75 \mathrm{ml} / \mathrm{min}$ with a mobile phase of acetonitrile/methanol/acetic acid 97:2:1 (v/v/v), with $5 \mathrm{mM}$ ammonium acetate 
(solvent A) and methanol/acetic acid 99:1 ( $\mathrm{v} / \mathrm{v} / \mathrm{v})$ with $5 \mathrm{mM}$ ammonium acetate (solvent B). LC separation and MS/MS detection were performed as previously described [36]. Ceramide and sphingomyelin species were quantified on the basis of calibration curves which were calculated from LC-MS/MS measurements of serially diluted synthetic ceramide and sphingomyelins standards (Avanti Polar Lipids, Alabaster, AL, USA). Linearity and correlation coefficients of the standard curves were obtained via linear regression analysis.

\section{Statistical Analysis}

Statistical analyses were performed using one-way ANOVA followed by the Tukey HSD test. Data are represented as means \pm SEM (animal studies) or means \pm SD (cell culture studies). $\mathrm{P}<0.05$ was considered statistically significant. 


\section{Results}

Feeding Angptl4 ${ }^{\digamma}$ mice a diet rich in saturated fatty acids but not trans- or cisunsaturated fatty acids results in peritonitis, chylous ascites and death.

Angptl4-/- mice were randomly assigned to a diet rich in saturated fatty acids ("Saturated"), a diet rich in cis-unsaturated fatty acids ("Unsaturated"), or a diet rich in trans-unsaturated fatty acids ("Trans"). Different fats were blended to achieve a similar relative content of saturated fatty acids between the cis-unsaturated and the transunsaturated diets, a similar content of cis-unsaturated fatty acids between the saturated and the trans-unsaturated diets, and a complete absence of trans fatty acids in the saturated and cis-unsaturated diets. Analysis of the fat composition of the three diets by gas chromatography indicated that approximately $60-65 \%$ of the total fatty acid content was constant among the three diets, with the remaining $35-40 \%$ of the fatty acids taken up by saturated fatty acids, cis-unsaturated fatty acids, or trans-unsaturated fatty acids (Figure 1A). The detailed fatty acid profiles of the three diets are shown in Supplemental Table 3. The Angptl4-/- mice were fed the diets for 7 weeks. Consistent with our previous studies, Angptl4-/- mice fed the saturated fat diet developed anorexia characterized by decreased feed intake (Figure 1B) and a concomitant steady decline in body weight (Figure 1C). Six of the 8 mice in the saturated fat group became severely ill and showed severe lethargy, reduced responsiveness to stimuli, and abdominal distention. These mice had to be euthanized before the end of the 7-week period, as illustrated by the survival curve (Figure 1D). Upon euthanasia, Angptl4-/- mice fed the saturated fat diet showed an exudate of chylous ascites and fibrinopurulent peritonitis, as previously reported (Figure 1E). In comparison with earlier experiments, the onset of the development of clinical symptoms in the Angptl4-/- mice was more rapid. Remarkably, the observed pathologies were absent both in the Angptl4-/- mice fed the cis or trans-unsaturated fat diet, even after more prolonged feeding (18 weeks) and in the wildtype mice fed any of the three diets.

After 2 weeks of feeding, prior to becoming visibly ill, plasma glucose levels were significantly lower in the Angptl4-/- mice fed the saturated fat diet compared to the other two groups (Figure 1F). By contrast, plasma insulin levels were the highest in the Angptl4-/- mice fed the trans-unsaturated fat diet. 


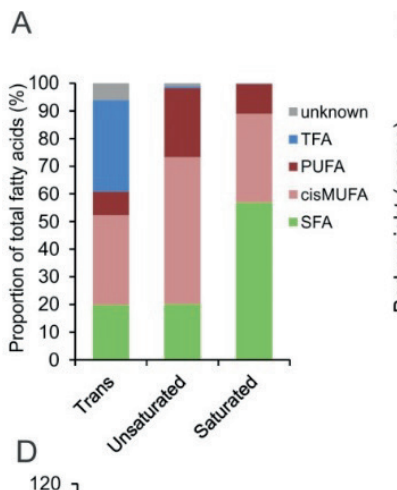

B

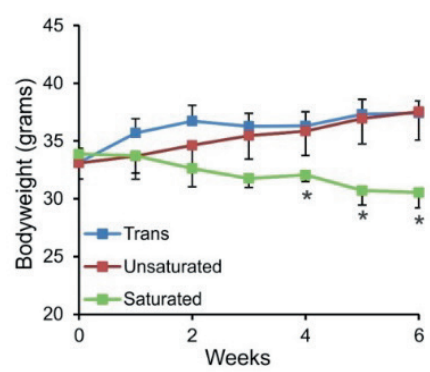

$E$
C

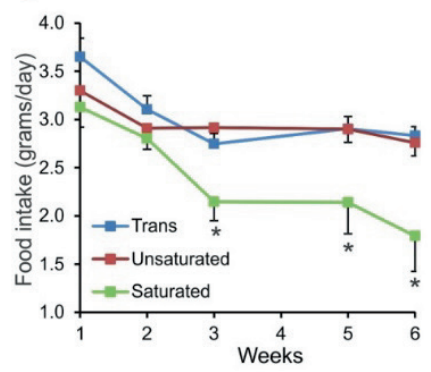

$\mathrm{F}$
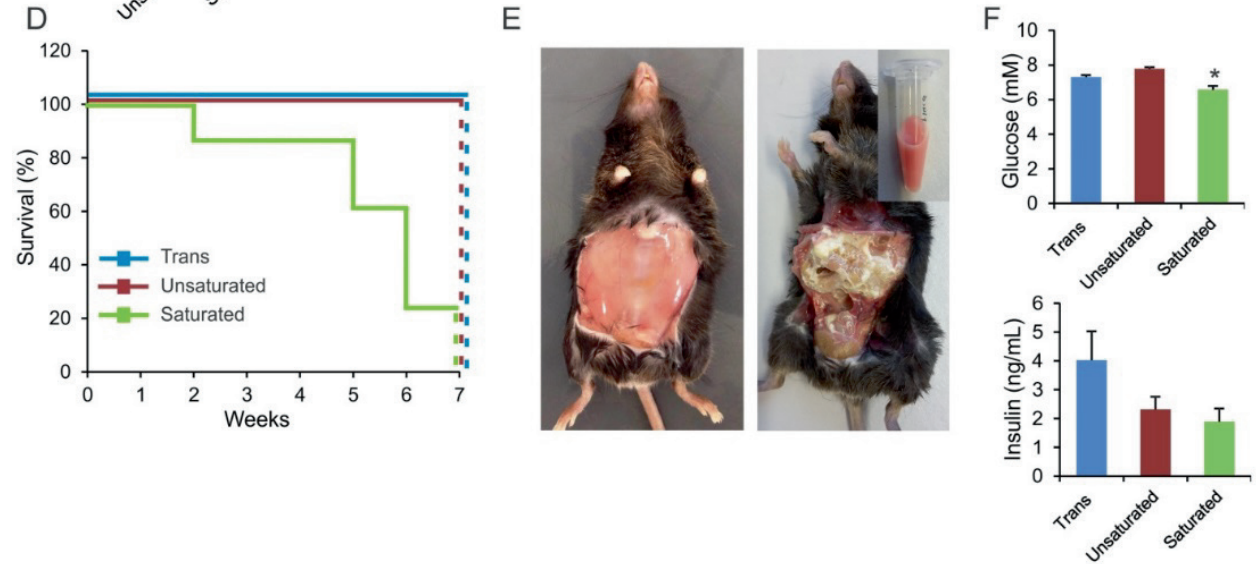

Figure 1: Feeding Angptl4 $\digamma$ mice a diet rich in saturated but not trans- or cis-unsaturated fatty acids results in peritonitis, chylous ascites and death. (A) Profile of fatty acid composition in the three test diets. Changes in body weight (B), average daily food intake (C), and survival (D) of Angptl4 $\%$ mice fed the trans, unsaturated or saturated fat diet for 7 weeks. (E) Representative pictures of Angptl4 $\uparrow$ mice fed the saturated fat diet immediately after sacrifice, before (left) and after (right) removal of peritoneum. A sample of the ascites fluid collected from the peritoneum of a single mouse is shown at the top right. (F) Plasma levels of glucose and insulin after 2 weeks of feeding. Error bars represent SEM. Asterisks indicates significantly different relative to the trans fat group $(* \mathrm{p}<0.05)$.

Feeding Angptl4 $/$ mice a diet rich in saturated fatty acids but not trans- or cisunsaturated fatty acids causes marked elevation of acute phase proteins.

To examine the effect of the three diets on inflammation, we measured plasma levels of the acute phase protein Serum Amyloid A (SAA). Strikingly, whereas Angptl4-/- mice fed the saturated fat diet showed a more than 50-fold increase in plasma SAA within one week, the mice fed the trans- and cis-unsaturated fat diet showed no increase at all (Figure 2A). 
A

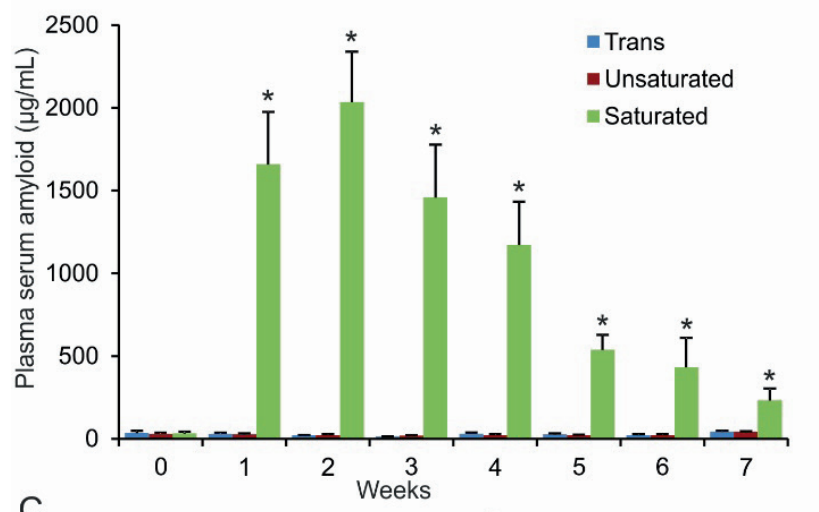

B

C

D
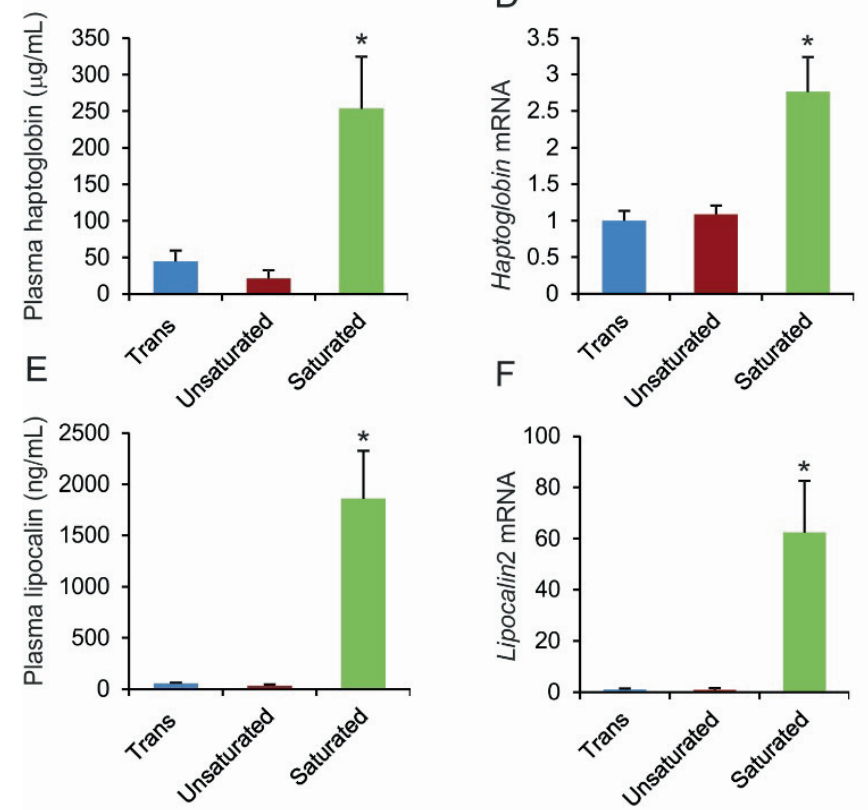

Figure 2: Feeding Angptl4 $/$ mice a diet rich in saturated but not trans- or cis-unsaturated fatty acids causes marked elevation of acute phase proteins. Angptl $\digamma$ mice were fed the trans, unsaturated or saturated fat diet for 7 weeks. (A) Weekly circulating levels of serum amyloid A (SAA). (B) Relative hepatic mRNA level of Saa2. (C) Plasma levels of haptoglobin. (D) Relative hepatic mRNA level of $H p$. (E) Plasma levels of Lipocalin. (F) Relative hepatic mRNA level of Lcn2. mRNA expression levels were normalised against 36b4. Please note that the Angptl4 $\digamma$ mice fed the saturated fat diet were euthanized at different time points due to the implementation of humane endpoints. Error bars represent SEM. Asterisks indicates significantly different relative to trans fat group $(* \mathrm{p}<0.05)$. 
In agreement with these data, liver mRNA levels of Saa2 at sacrifice were significantly elevated in the saturated fat group but not in the trans- or cis-unsaturated fat groups (Figure 2B). Plasma levels of haptoglobin and lipocalin, as well as liver mRNA of $H p$ and $L c n 2$, mirrored the SAA data, showing a marked increase in the saturated fat group in comparison to both the trans- or cis-unsaturated fat groups (Figure 2C-F). Overall, these data show that feeding Angptl4-/- mice a diet rich in trans-unsaturated fatty acids does not lead to chylous ascites and peritonitis, and does not provoke a massive acute phase response, unlike Angptl4-/- mice fed a diet rich in saturated fatty acids.

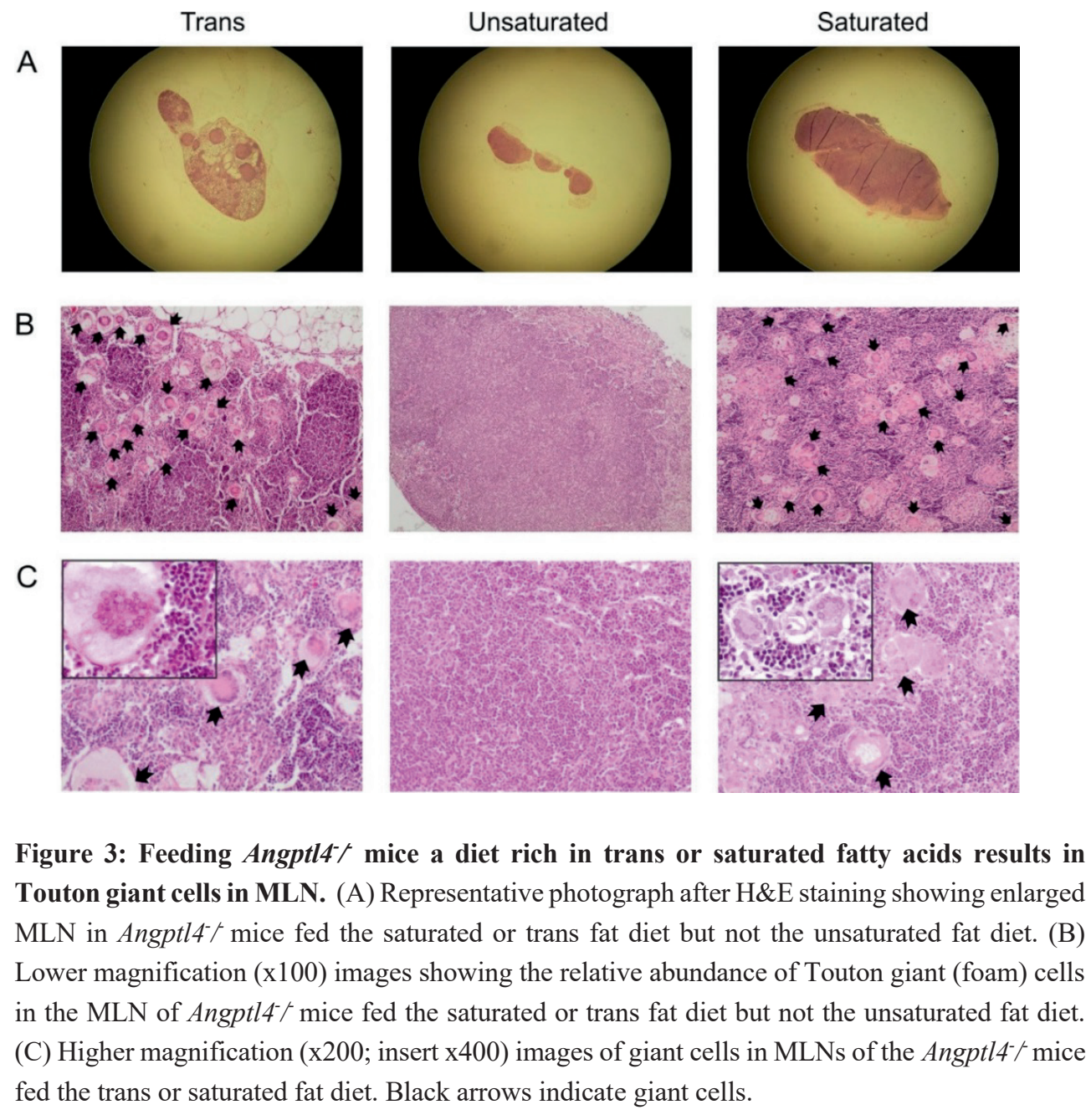


Feeding Angptl4 $\%$ mice a diet rich in trans or saturated fatty acids stimulates lymphadenopathy characterized by formation of giant cells.

We have previously demonstrated that the development of chylous ascites in Angptl4-/mice fed a saturated fat diet is accompanied by mesenteric lymphadenopathy as shown by enlarged MLN and the appearance of Touton giant cells (macrophage foam cells). Therefore, in this study, we performed H\&E staining on the lymph nodes to examine giant cell formation. In agreement with our earlier studies, lipid-laden giant cells could be observed in the enlarged MLN of Angptl4-/- mice fed the saturated fat diet ( 6 positives out of 7 lymph nodes studied). Intriguingly, despite the absence of ascites and any elevation in plasma acute phase proteins, MLN were enlarged and giant cells were also plentiful in the Angptl4-/- mice fed the trans-unsaturated fat diet (9 positives out of 13 lymph nodes studied) (Figure 3A, B and C). No giant cells were observed in the MLN of Angptl4-/- mice fed the cis-unsaturated fat diet ( 0 positives out of 5 lymph nodes studied) nor were they ever observed in wildtype mice. A summary of the observations in the Angptl4-/- mice fed the three diets is presented in Supplemental Table 4. These data show that mesenteric lymphadenopathy in Angptl4-/- mice is uncoupled from the hepatic acute phase response and chylous ascites.

\section{Palmitate but not elaidate and oleate stimulates UPR in cultured macrophages.}

The experiments in Angptl4-/- mice suggest that saturated fatty acids and transunsaturated fatty acids have distinct effects on inflammation, despite both leading to foam cell formation. To study the mechanistic basis for these differences in inflammatory properties, we used RAW264.7 macrophages and incubated these cells either with a saturated fatty acid (palmitate), a cis-unsaturated fatty acid (oleate), or a transunsaturated fatty acid (elaidate). Oil red-O staining showed that all three fatty acids promoted lipid droplet and foam cell formation (Figure 4A). Histological and biochemical analysis did not reveal any differences in lipid accumulation among the three fatty acids (Figure 4B). Consistent with the in vivo data, the saturated fatty acid palmitate had a pronounced pro-inflammatory effect, as shown by the induction of numerous genes involved in inflammation, including Ptgs2, Cxcl2 and Gdf15, whereas oleate and elaidate caused a modest yet significant decrease in inflammatory gene expression (Figure 5A). The differences in inflammatory properties of the fatty acids could not be explained by differences in activation of oxidative stress, as determined by measuring ROS production (Figure 5B). 
A
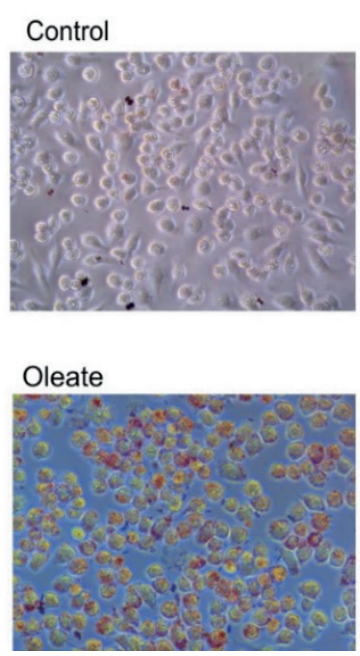

Elaidate

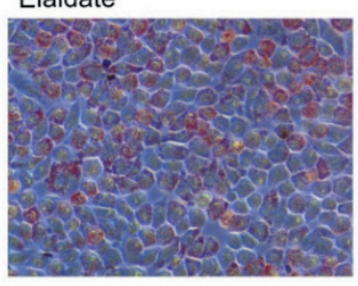

Palmitate

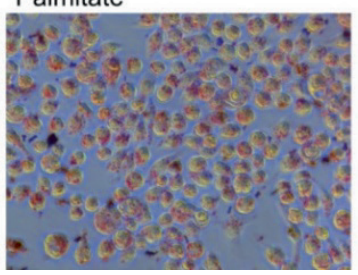

B

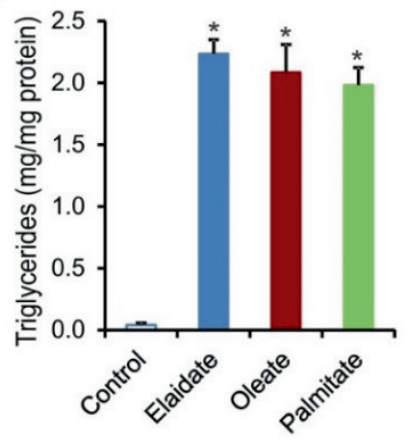

Figure 4: Similar lipid content in RAW264.7 macrophages treated with different fatty acids.

RAW264.7 macrophages were treated with $500 \mu \mathrm{M}$ of individual fatty acids (palmitate, oleate or elaidate) or vehicle control for 6 hours. (A) Representative images of Oil red-O staining of lipid droplets in RAW264.7 macrophages. (B) Intracellular triglyceride quantification after fatty acid treatments. Error bars represent SD. Asterisks indicate statistically significant relative to vehicle control $(* \mathrm{p}<0.05)$.

To gain insight into the mechanism(s) underlying the differential properties of palmitate and elaidate, we performed whole genome expression profiling. Visualization of the overall gene expression data by heat map showed that the impact of palmitate on gene expression was very distinct from that of oleate and elaidate (Figure 5C). In turn, the effects of elaidate and oleate were quite similar with the exception of a cluster of genes involved in lipid and cholesterol synthesis. When related to oleate, palmitate caused much more pronounced changes in gene expression than elaidate, as shown by scatter plot analysis (Figure 5D). Ingenuity pathway analysis on genes induced by palmitate further corroborated the profound stimulation of inflammatory genes by palmitate. In particular, genes part of the pathway "Communication between Innate and Adaptive Immune Cells" were highly upregulated by palmitate (rank 6), and to a much lesser extent by elaidate (Figure 5E). By contrast, the pathway "Superpathway of Cholesterol Biosynthesis" was specifically induced by elaidate (rank 1) (Figure 5G). Interestingly, the pathway "Unfolded Protein Response" was highly induced by palmitate (rank 3) when compared with elaidate and oleate (Figure 5F). 
A B

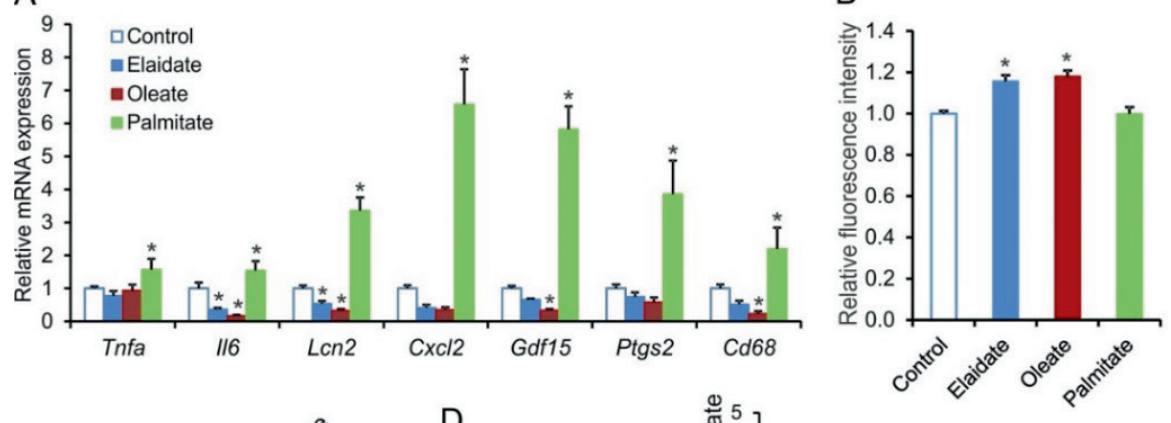

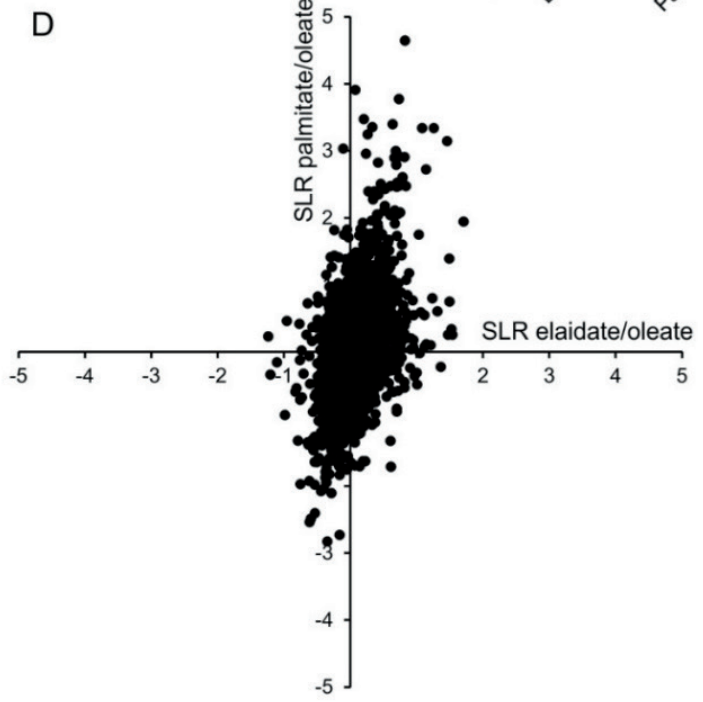
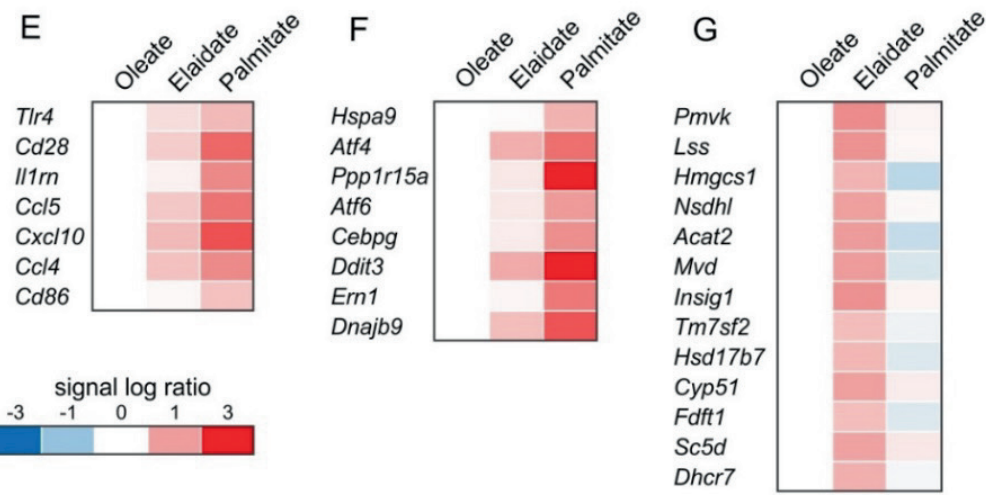

Figure 5: Differential gene expression profiles in macrophages treated with palmitate, oleate or elaidate. (A) Q-PCR expression of selected inflammatory markers in RAW264.7 cells after treatment with $500 \mu \mathrm{M}$ fatty acids or vehicle control for 6 hours. (B) Fluorometric measurement of relative reactive oxygen species as marker of oxidative stress in RAW264.7 macrophages. (C) Heatmap of the 500 most variable genes based on the calculation of the coefficient of variation. 
The cluster of genes deviating in the elaidate-treated cells is indicated. (D) Scatter plot of genes that passed the UPC filter (7905 of the 22135 probesets). The signal log ratio for the comparison of palmitate vs. oleate treated cells is shown along the Y-axis. The signal log ratio for the comparison of elaidate vs. oleate treated cells is shown along the X-axis. (E-G) Heatmaps of pathways that were induced by palmitate: communication between innate and adaptive immune cells (E) and unfolded protein response (F), or elaidate: superpathway of cholesterol biosynthesis $(\mathrm{G})$, according to Ingenuity pathway analysis. Asterisks indicate statistically significant relative to vehicle control $(* \mathrm{p}<0.05)$.

Activation of the unfolded protein response (UPR) may provide a mechanistic basis for the pro-inflammatory action of palmitate. UPR maintains cell survival via activation of sensors such as protein kinase RNA-like ER kinase (PERK), activating transcription factor 6 (ATF6) and inositol requiring kinase 1 (IRE1), which in turn triggers the unconventional splicing of Xbpl mRNA to generate the transcription factor XBP1s [3740]. Detailed analyses showed that palmitate increased numerous indicators of UPR, including XBP1 splicing (Figure 6A), IRE1 $\alpha$ phosphorylation, PERK phosphorylation, $\mathrm{XBP} 1$ and $\mathrm{CHOP}$ protein levels (Figure 6B), and expression of various ER stress and UPR downstream effector/responsive genes (Figure 6C), which was not observed for oleate and elaidate. In fact, oleate and elaidate seemed to lead to a mild but significant reduction in UPR in general as compared to control cells, as indicated by IRE1 $\alpha$ phosphorylation, Ddit3 and Grp78 mRNA levels, and Xbpl alternative splicing. In addition, palmitate induced cleavage of caspase 3 , concurrent with upregulation of cell stress markers, suggesting enhanced apoptosis (Figure 6D). None of these fatty acids caused the induction of ER-associated degradation proteins SEL1L and HRD1 under these experimental conditions (Figure 6E). To verify that the behavior of elaidate is representative of a broader group of trans unsaturated fatty acids, we studied the effect of trans-vaccenate, palmitelaidate, 9Z,11E conjugated linoleate, and 10E,12Z conjugated linoleate in RAW264.7 macrophages. All trans fatty acids led to a similar expression level of a selected group of UPR and inflammation gene markers (Figure 6F), and all were similarly ineffective towards inducing CHOP and XBP1 protein levels (Figure 6G), in clear contrast to palmitate. Aside from palmitate, the other saturated fatty acids laurate, myristate and stearate effectively induced UPR and inflammation marker genes as well as $\mathrm{CHOP}$ and $\mathrm{XBP} 1$ protein levels, with a seemingly positive correlation between the magnitude of induction and the length of the fatty acid chain (Figure 6H and I). These data show that the observed effects of palmitate and elaidate are representative of a larger set of saturated and trans unsaturated fatty acids, respectively. 
A Elaidate Oleate Palmitate Control C

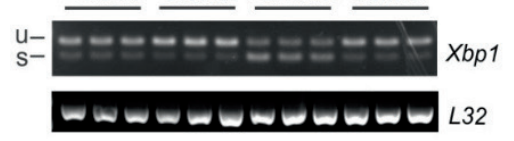

B

Elaidate Oleate Palmitate Control
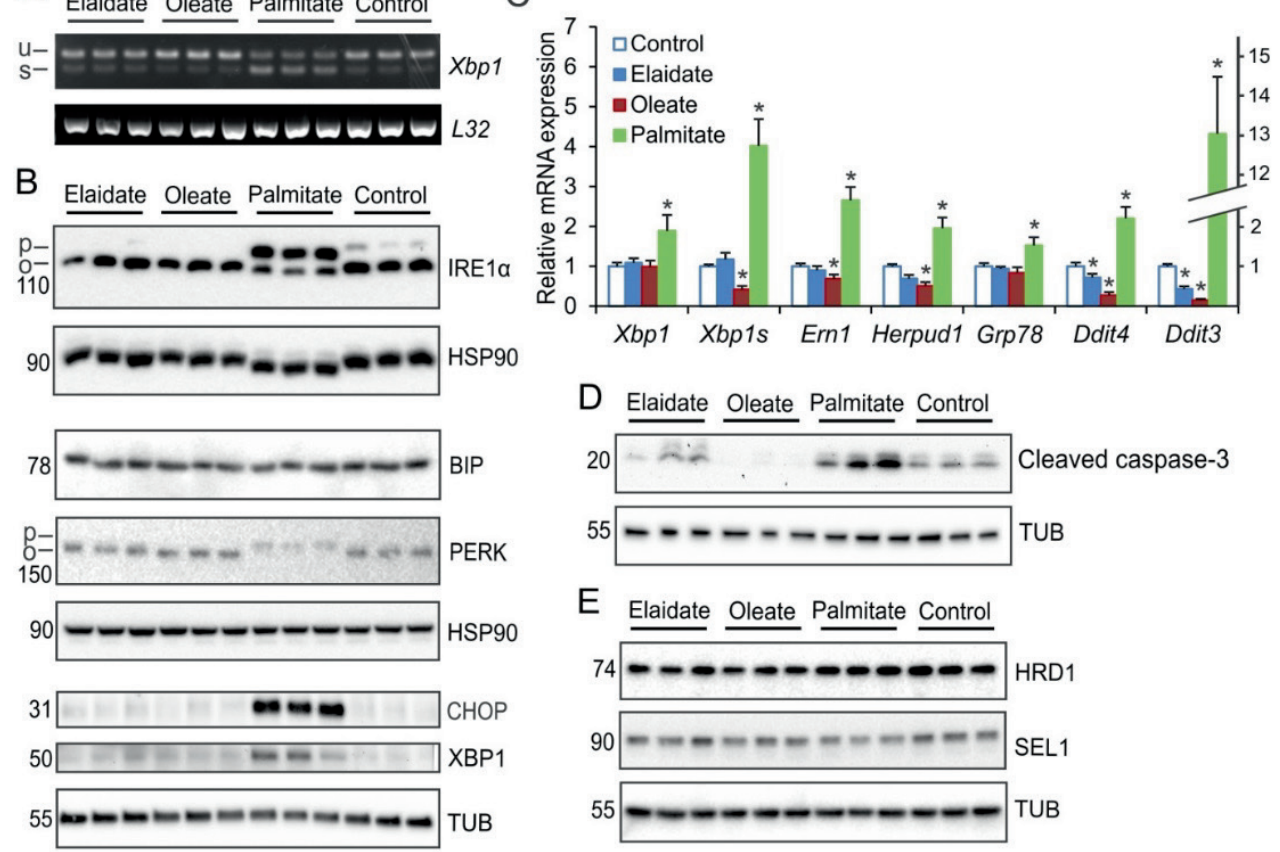

D Elaidate Oleate Palmitate Control

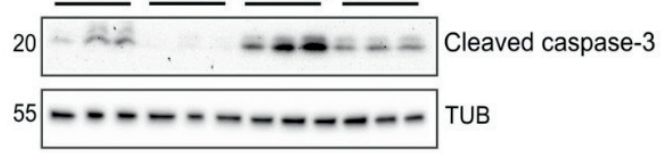

E Elaidate Oleate Palmitate Control

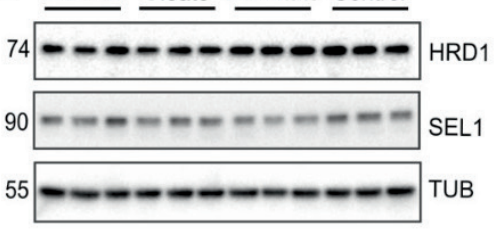

$\mathrm{F}$
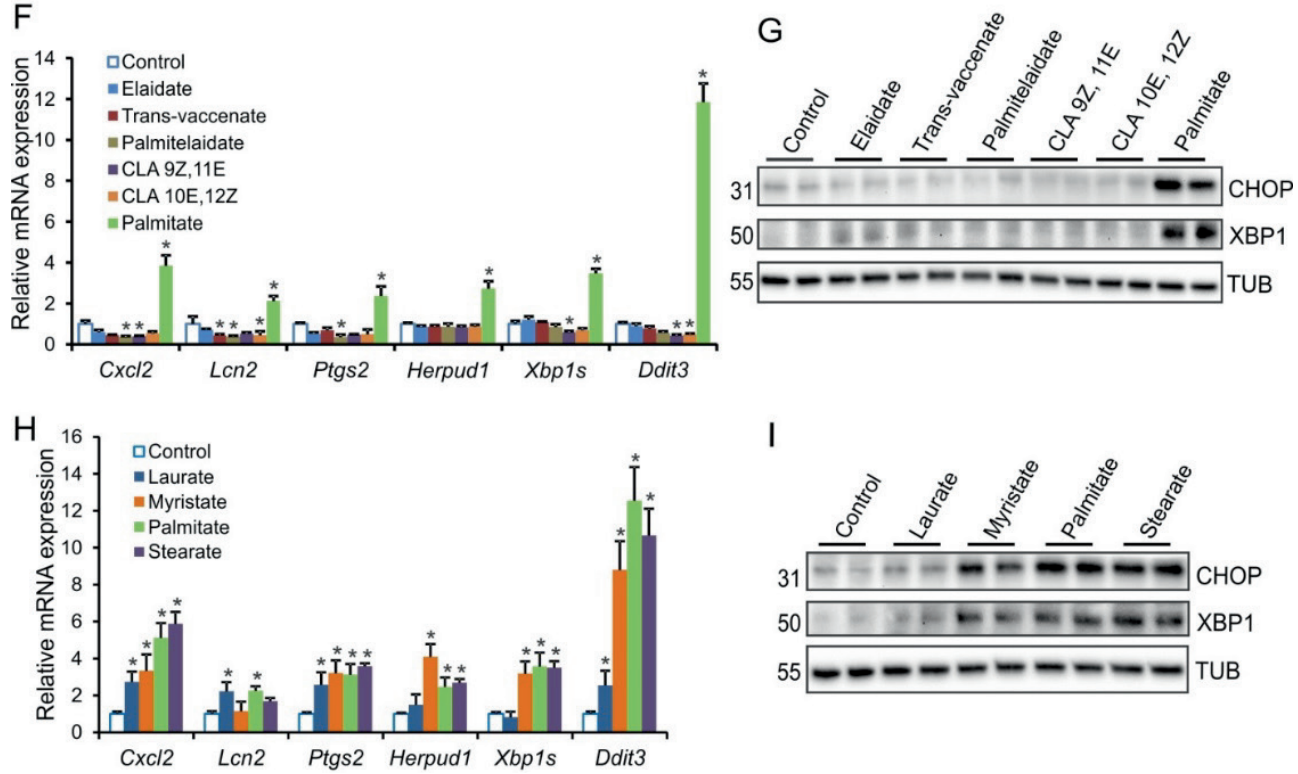

Figure 6: Palmitate but not elaidate or oleate induces UPR and inflammation markers in RAW264.7 macrophages. RAW264.7 macrophages were treated for 6 hours with individual fatty acids $(500 \mu \mathrm{M})$ as indicated. (A) RT-PCR of Xbpl processing showing unspliced(u) and spliced(s) Xbp1 mRNA with L32 as control. (B) Immunoblot of UPR downstream effectors and 
UPR responsive proteins using regular gels (BIP, PERK, CHOP and XBP1) and phospho-tag gels (IRE1 $\alpha$ ). (C) Q-PCR expression of selected UPR markers. (D) Immunoblot of cleaved caspase-3, an indicator of activated cell death by apoptosis. (E) Immunoblot of endoplasmic reticulumassociated protein degradation markers. (F) Q-PCR expression of selected UPR and inflammation marker genes after treatment with the trans fatty acids trans-vaccenic acid, palmitelaidic acid, 9Z,11E conjugated linoleic acid (CLA 9Z, 11E), 10E,12Z conjugated linoleic acid (CLA 10E, $12 \mathrm{Z}$ ), and palmitate. (G) Immunoblot of CHOP and XBP1 after treatment with different trans fatty acids and palmitate. $(\mathrm{H})$ Q-PCR expression of selected UPR and inflammation marker genes after treatment with the saturated fatty acids laurate, myristate, palmitate, and stearate. (I) Immunoblot of CHOP and XBP1 after treatment with different saturated fatty acids. mRNA expression was normalised against 36b4. Error bars represent SD. Asterisks indicate statistically significant relative to vehicle control $(* \mathrm{p}<0.05)$.

Consistent with the results shown above, palmitate markedly raised levels of the lipotoxic mediator ceramide as compared to elaidate and oleate, as reflected by the total ceramide levels (Figure 7A) and levels of fatty-acyl subspecies (Figure 7B). To better delineate the differential lipotoxic effects of saturated and unsaturated fatty acids, we tested the effect of a number of chemical inhibitors of various pathways of intracellular lipid metabolism - including the ceramide pathway - on palmitate-induced UPR and inflammation in RAW264.7 macrophages. Whereas most compounds showed minimal effects, the CD36 inhibitor sulfosuccinimidyl oleate markedly suppressed the induction of $\mathrm{Cxcl} 2$ and Ddit3 by palmitate, suggesting that cellular fatty acid uptake is necessary for palmitate-induced lipotoxicity (Figure 7C). By contrast, the sphingosine kinase inhibitor dimethylsphingosine and the acyl-CoA synthetase inhibitor triascin C markedly augmented the induction of $\mathrm{Cxcl} 2$ and Ddit3 by palmitate (Figure 7C). Accordingly, we selected these two compounds to further dissect the differential effects of trans and saturated fatty acids. Interestingly, in the presence of dimethylsphingosine or triascin $\mathrm{C}$, elaidate and oleate lowered expression of UPR and inflammation markers, indicating an anti-lipotoxic action (Figure 7D). This anti-lipotoxic effect was most pronounced for oleate but also clearly noticeable for elaidate.

Overall, these data indicate that saturated fatty acids and trans fatty acids differentially affect UPR and inflammation in cultured macrophages. 

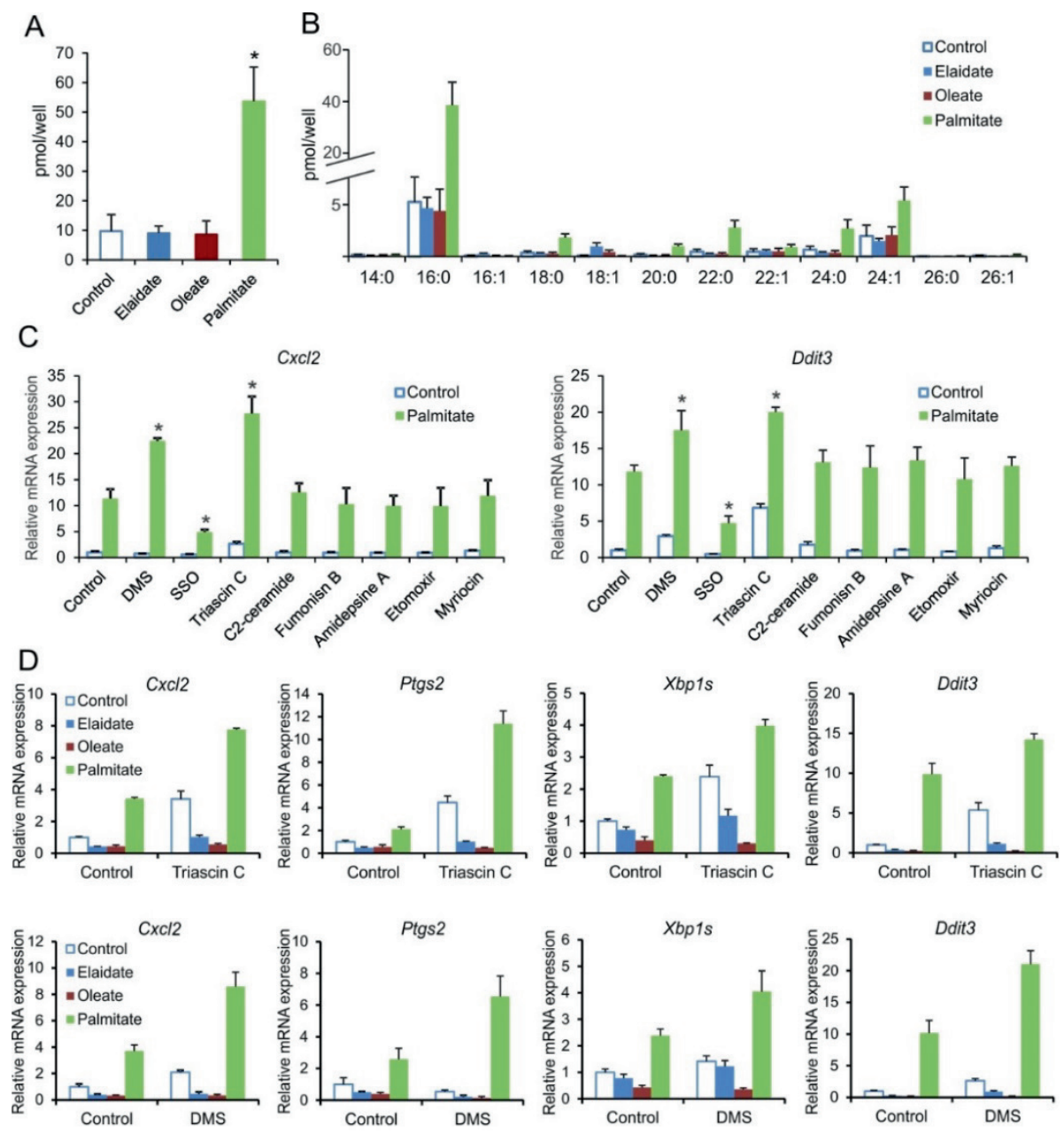

Figure 7: Anti-lipotoxic effect of oleate and elaidate in RAW264.7 macrophages. RAW264.7 macrophages were treated for 6 hours with individual fatty acids $(500 \mu \mathrm{M})$ as indicated. Total ceramide levels (A) and levels of ceramide fatty-acyl subspecies (B) after 6-hour treatment with elaidate, oleate, palmitate or vehicle control. (C) Cxcl2 and Ddit3 expression in RAW264.7 macrophages treated with palmitate or vehicle control and co-treated with the compounds dimethylsphingosine (DMS), sulfosuccinimidyl olate (SSO), triascin C, C2-ceramide, fumonisin B1, amidepsine A, etomoxir and myriocin. (D) Expression of selected UPR and inflammation markers in fatty acid-treated RAW264.7 cells co-treated with either triascin C (top row) or DMS (bottom row). mRNA expression was normalised against 36b4. Error bars represent SD. Asterisks indicate statistically significant relative to vehicle control $(* \mathrm{p}<0.05)$. 


\section{Discussion}

The work presented in this paper leads to two important conclusions. First, our data show that lipid accumulation and the appearance of giant cells in MLN (mesenteric lymphadenopathy) in Angptl4-/- mice fed a high fat diet is disconnected from activation of the acute phase response, chylous ascites, and death. Accordingly, the development of mesenteric lymphadenopathy upon ANGPTL4 inactivation may be clinically silent, both in the short and long term, removing part of the concern associated with the use of ANGPTL4-inactivating antibodies as lipid-lowering therapy. Second, we demonstrate that the effects of saturated fatty acids and trans fatty acids are very distinct, both in vivo and in cultured macrophages. Hence, our data do not support the common notion that trans fatty acids act like saturated fatty acids.

By virtue of their ability to inhibit LPL and raise circulating triglyceride levels, members of the Angiopoietin-like protein family are intensely explored as pharmacological targets for dyslipidemia [41-43]. Whereas the targeting of ANGPTL3 has been very successful [44,45] — with strategies to inactivate ANGPTL3 now having moved into clinical trial phase - the targeting of ANGPTL4 has been hampered by the observation that inactivating ANGPTL4 leads to severe clinical problems in mice fed a diet high in saturated fat. Specifically, inactivation of ANGPTL4 causes enhanced lipid uptake into macrophages in MLN, triggering foam (Touton) cell formation and a massive inflammatory response, which progresses into chylous ascites and ultimately death by peritonitis $[17,46]$.

Two recent genetic studies generated new interest in ANGPTL4 as lipid-lowering target by showing that human carriers of an inactivating mutant of ANGPTL4 have a lower risk of coronary artery disease than non-carriers, concomitant with a reduction in serum triglycerides $[15,16]$. However, one of these studies also found accumulation of lipids in the MLN in a number of the female monkeys treated with ANGPTL4-inactivating antibodies, copying the symptoms seen in Angptl4-/- mice fed a high saturated fat diet [16]. These findings raised concern about the possible side-effects of the use of ANGPTL4-inactivating antibodies in humans. In this paper we show that lipid accumulation and the appearance of lipid-laden giant cells in MLN is uncoupled from the activation of a massive pro-inflammatory response. In particular, we find that despite developing giant cells in their MLN, Angptl4-/- mice fed a diet rich in trans fatty acids do not have elevated plasma serum amyloid and haptoglobin levels, do not develop ascites, and survive, unlike Angptl4-/- mice fed a diet rich in saturated fatty acids. Our findings thus imply that the presence of giant cells in MLN is non-deleterious and 
clinically silent, which, together with the reduced risk of coronary artery disease in E40K carriers, suggests that ANGPTL4 may still have promise as a viable therapeutic target for triglyceride lowering and coronary artery disease. To predict future clinical problems, our data also emphasize the need to closely monitor plasma levels of acute phase proteins, both in E40K homozygotes as well as in monkeys and patients that might be treated with ANGPTL4-inactivating antibodies.

Previously, we proposed that elevated LPL activity in MLN macrophages is responsible for the formation of lipid-laden giant cells in Angptl4-/- mice fed a high saturated fat diet [17]. We hypothesized that the lipid-activated macrophages would release proinflammatory cytokines into the portal circulation that activate the hepatic acute phase response. The data presented in this paper indicate that the formation of lipid-laden giant cells per se is insufficient to initiate an acute phase response, thus pointing towards an alternative and/or additional mechanism. Based on our in vitro studies, this mechanism requires uptake of fatty acids, is specifically activated by saturated fatty acids, and may be linked to the ceramide pathway (see below). Interestingly, Angptl4-/- mice do not develop lipid-laden giant cells in MLN when fed a diet rich in cis-unsaturated fatty acids, even though oleate was equally effective at promoting lipid accumulation in RAW264.7 macrophages. It can be speculated that the development of lipid-laden giant cells in vivo may be dependent on concurrent activation of macrophage or other immune cells, which is triggered by saturated fatty acids (and trans-unsaturated fatty acids) but not by the more anti-inflammatory cis-unsaturated fatty acids.

Because trans and saturated fatty acids have a similar linear structure, trans fatty acids are claimed to act like saturated fatty acids. Indeed, nutritional trials have shown that consumption of diets rich in saturated fatty acid and trans fatty acids both raise LDLcholesterol levels in human $[23,47]$. However, to what extent saturated and trans fatty acids exert the same effects at the cellular level and share the same mechanism of action is unclear. Our results indicate that the biological effects of saturated and trans fat in Angptl4-/- mice are very different. Whereas feeding a diet rich in saturated fat provoked a pronounced inflammatory response characterized by a huge increase in plasma levels of acute phase proteins, no such response was seen in Angptl4-/- mice fed a diet rich in trans fatty acids. Experiments in RAW267.4 macrophages confirmed the distinct biological effects of the saturated fatty acid palmitate as compared to the trans fatty acid elaidate. Specifically, whereas palmitate triggered inflammation, UPR, and apoptosis, elaidate induced genes involved in cholesterol synthesis. The properties of palmitate and elaidate were shown to be representative of a whole class of saturated fatty acids and trans fatty acids, respectively. 
The observed lipotoxic properties of palmitate are in line with numerous published studies performed in several cell types where palmitate induced a number of stressrelated pathways such as oxidative stress, inflammation, and UPR [48-52]. Several physiological and pathological insults can disturb ER function, leading to the accumulation of unfolded proteins and causing the subsequent activation of UPR. In our studies in RAW264.7 macrophages, we found that palmitate treatment potently activated UPR and inflammatory gene expression. By contrast, elaidate and especially oleate suppressed UPR and pro-inflammatory gene expression, which was most evident when baseline levels of UPR and inflammation were raised using dimethylsphingosine or triascin C. These observations are consistent with existing evidence indicating that saturated and cis-unsaturated fatty acids are pro-lipotoxic and anti-lipotoxic, respectively [50,53-55]. Studies have indicated that palmitate may induce ER stress by altering the ER-membrane composition. Indeed, there is evidence that direct sensing of the lipid composition of the ER membrane contributes to the UPR [56,57]. Specifically, it has been suggested that saturated acyl chains are less flexible than unsaturated acyl chains and change the biophysical properties of the membrane to favor dimerization and concomitant activation of IRE1 and PERK [58,59]. If that is true, elaidate would be expected to mimic the properties of palmitate, which was not at all observed. Consistent with our observations, trans-unsaturated fatty acids did not induce ER stress or cytotoxicity in yeast and $\beta$ cells $[60,61]$. The modest repressive effect of trans fatty acids on well-established indicators of lipotoxicity such as UPR and inflammation is both unexpected and interesting and presents an attractive challenge for further investigation into the potential specific molecular mechanisms behind the purported harmful effects of trans fat. Overall, these data underscore the distinct properties of saturated and transunsaturated fatty acids.

Treatment of RAW264.7 macrophages with palmitate caused a marked increase in levels of ceramide, which is a known lipotoxic intermediate. However, our studies using a ceramide analog and inhibitors of ceramide synthesis suggest that ceramide itself may not be responsible for the palmitate-induced lipotoxicity. The observed potentiation of the lipotoxic effect of palmitate by dimethylsphingosine has been observed previously [62], and suggests a potential role of sphingosine or sphingosine-1-phosphate. Besides dimethylsphingosine, the acyl-CoA synthesis inhibitor triascin $\mathrm{C}$ also potentiated palmitate-induced lipotoxicity. This result is in line with a similar study in mouse peritoneal macrophages treated with stearate [63], and suggest that lipotoxicity is mediated by free palmitate/stearate. Based on our experiments, it is not possible to attribute the lipotoxic effects of palmitate to a single well-defined mechanism. 
In conclusion, our data indicate that the presence of lipid-laden giant cells in MLN is uncoupled from the acute phase response and chylous ascites. The probable nondeleterious effect of foam cells in MLN of ANGPTL4 deficient mice suggests that ANGPTL4 should not be fully dismissed as a target for correcting dyslipidemia and atherosclerosis in humans. Finally, our in vitro and in vivo data strongly indicate that trans and saturated fatty acids have distinct biological effects, with the trans fatty acids leaning much more towards cis-unsaturated fatty acids than towards saturated fatty acids. 
Acknowledgments: All authors edited and revised the manuscript and approved the final version. Sander Kersten $(\mathrm{PhD})$ is responsible for the integrity of the work as a whole.

Funding: This work was supported by a Nanyang Technological University iFood Research Grant (WBS M4081459.080), by Fondation Leducq Grant 12CVD04, and by the Graduate School VLAG (Wageningen University).

Disclosure: The authors declare that there is no conflict of interest associated with this manuscript. 


\section{References}

[1] Wang, H., Eckel, R.H., 2009. Lipoprotein lipase: from gene to obesity. American Journal of Physiology. Endocrinology and Metabolism 297(2): E271-88.

[2] Investigators, M.I.G. and Cardi.E.C., 2016. Coding Variation in ANGPTL4 , LPL, and SVEP1 and the Risk of Coronary Disease. New England Journal of Medicine 374(12).

[3] Fong, L.G., Young, S.G., Beigneux, A.P., Bensadoun, A., Oberer, M., Jiang, H., et al., 2016. GPIHBP1 and Plasma Triglyceride Metabolism. Trends in Endocrinology \& Metabolism xx(7): 1-15.

[4] Beigneux, A.P., Franssen, R., Bensadoun, A., Gin, P., Melford, K., Peter, J., et al., 2009. Chylomicronemia with a mutant GPIHBP1 (Q115P) that cannot bind lipoprotein lipase. Arteriosclerosis, Thrombosis, and Vascular Biology 29(6): 95662.

[5] Beigneux, A.P., Davies, B.S.J., Gin, P., Weinstein, M.M., Farber, E., Qiao, X., et al., 2007. Glycosylphosphatidylinositol-Anchored High-Density Lipoprotein-Binding Protein 1 Plays a Critical Role in the Lipolytic Processing of Chylomicrons. Cell Metabolism 5(4): 279-91.

[6] Dijk, W., Kersten, S., 2014. Regulation of lipoprotein lipase by Angpt14. Trends in Endocrinology and Metabolism 25(3): 146-55.

[7] Catoire, M., Alex, S., Paraskevopulos, N., Mattijssen, F., Evers-van Gogh, I., Schaart, G., et al., 2014. Fatty acid-inducible ANGPTL4 governs lipid metabolic response to exercise. Proceedings of the National Academy of Sciences of the United States of America 111(11): E1043-52.

[8] Kersten, S., Mandard, S., Tan, N.S., Escher, P., Metzger, D., Chambon, P., et al., 2000. Characterization of the fasting-induced adipose factor FIAF, a novel peroxisome proliferator-activated receptor target gene. Journal of Biological Chemistry 275(37): 28488-93.

[9] Yoon, J.C., Chickering, T.W., Rosen, E.D., Dussault, B., Qin, Y., Soukas, A., et al., 2000. Peroxisome proliferator-activated receptor gamma target gene encoding a novel angiopoietin-related protein associated with adipose differentiation. Molecular and Cellular Biology 20(14): 5343-9.

[10] Dijk, W., Beigneux, A.P., Larsson, M., Bensadoun, A., Young, S.G., Kersten, S., 2016. Angiopoietin-like 4 (ANGPTL4) promotes intracellular degradation of lipoprotein lipase in adipocytes. Journal of Lipid Research 7(11): 956-63.

[11] Sukonina, V., Lookene, A., Olivecrona, T., Olivecrona, G., 2006. Angiopoietin-like protein 4 converts lipoprotein lipase to inactive monomers and modulates lipase activity in adipose tissue. Pnas 103(46): 17450-5.

[12] Mandard, S., Zandbergen, F., Van Straten, E., Wahli, W., Kuipers, F., Müller, M., 
et al., 2006. The fasting-induced adipose factor/angiopoietin-like protein 4 is physically associated with lipoproteins and governs plasma lipid levels and adiposity. Journal of Biological Chemistry 281(2): 934-44.

[13] Köster, A., Chao, Y.B., Mosior, M., Ford, A., Gonzalez-DeWhitt, P.A., Hale, J.E., et al., 2005. Transgenic angiopoietin-like (Angptl)4 overexpression and targeted disruption of Angptl4 and Angptl3: Regulation of triglyceride metabolism. Endocrinology 146(11): 4943-50.

[14] Sonnenburg, W.K., Yu, D., Lee, E.-C., Xiong, W., Gololobov, G., Key, B., et al., 2009. GPIHBP1 stabilizes lipoprotein lipase and prevents its inhibition by angiopoietin-like 3 and angiopoietin-like 4. Journal of Lipid Research 50(12): 24219.

[15] Abid, K., Trimeche, T., Mili, D., Msolli, M.A., Trabelsi, I., Nouira, S., et al., 2016. ANGPTL4 variants E40K and T266M are associated with lower fasting triglyceride levels and predicts cardiovascular disease risk in Type 2 diabetic Tunisian population. Lipids in Health and Disease 15(1): 63.

[16] Dewey, F.E., Gusarova, V., O'Dushlaine, C., Gottesman, O., Trejos, J., Hunt, C., et al., 2016. Inactivating Variants in ANGPTL4 and Risk of Coronary Artery Disease. New England Journal of Medicine 374(12): 1123-33.

[17] Lichtenstein, L., Mattijssen, F., de Wit, N.J., Georgiadi, A., Hooiveld, G.J., van der Meer, R., et al., 2010. Angptl4 Protects against Severe Proinflammatory Effects of Saturated Fat by Inhibiting Fatty Acid Uptake into Mesenteric Lymph Node Macrophages. Cell Metabolism 12(6): 580-92.

[18] Bassett, C.M.C., Edel, A.L., Patenaude, A.F., McCullough, R.S., Blackwood, D.P., Chouinard, P.Y., et al., 2010. Dietary vaccenic acid has antiatherogenic effects in LDLr-/- mice. The Journal of Nutrition 140(1): 18-24.

[19] Stender, S., Astrup, A., Dyerberg, J., 2008. Ruminant and industrially produced trans fatty acids: Health aspects. Food and Nutrition Research 52: 1-8.

[20] Mozaffarian, D., Katan, M.B., Ascherio, A., Stampfer, M.J., Willett, W.C., 2006. Trans Fatty Acids and Cardiovascular Disease. New England Journal of Medicine 354(15): 1601-13.

[21] Ganguly, R., Pierce, G.N., 2012. Trans fat involvement in cardiovascular disease. Molecular Nutrition and Food Research 56(7): 1090-6.

[22] Ginter, E., Simko, V., 2016. New data on harmful effects of trans-fatty acids. Bratislavské Lekárske Listy 117(5): 251-3.

[23] de Souza, R.J., Mente, A., Maroleanu, A., Cozma, A.I., Ha, V., Kishibe, T., et al., 2015. Intake of saturated and trans unsaturated fatty acids and risk of all cause mortality, cardiovascular disease, and type 2 diabetes: systematic review and metaanalysis of observational studies. BMJ (Clinical Research Ed.) 351(6): h3978.

[24] WILLETT, W., 1993. Intake of trans fatty acids and risk of coronary heart disease among women. The Lancet 341(8845): 581-5. 
[25] Eckel, R.H., Borra, S., Lichtenstein, A.H., Yin-Piazza, S.Y., 2007. Understanding the complexity of trans fatty acid reduction in the American diet: American heart association trans fat conference 2006: Report of the trans fat conference planning group. Circulation 115(16): 2231-46.

[26] Kaur, G., Cameron-Smith, D., Sinclair, A.J., 2012. Are trans fats a problem in Australia? The Medical Journal of Australia 196(11): 666-7.

[27] L'Abbé, M.R., Stender, S., Skeaff, C.M., Tavella, M., 2009. Approaches to removing trans fats from the food supply in industrialized and developing countries. European Journal of Clinical Nutrition 63: S50-67.

[28] Stender, S., Dyerberg, J., Astrup, A., 2006. Consumer protection through a legislative ban on industrially produced trans fatty acids in foods in Denmark. Scandinavian Journal of Food \& Nutrition 50(July): 155-60.

[29] Pérez-Farinós, N., Dal Re Saavedra, M.Á., Villar Villalba, C., Robledo de Dios, T., 2016. Trans-fatty acid content of food products in Spain in 2015. Gaceta Sanitaria 30(5): 379-82.

[30] Folch, J., Lees, M., Sloane Stanley, G.H., 1957. A simple method for the isolation and purification of total lipides from animal tissues. The Journal of Biological Chemistry 226(1): 497-509.

[31] Yang, L., Xue, Z., He, Y., Sun, S., Chen, H., Qi, L., 2010. A phos-tag-based approach reveals the extent of physiological endoplasmic reticulum stress. PLoS ONE 5(7): 1-7.

[32] Irizarry, R.A., Hobbs, B., Collin, F., Beazer-Barclay, Y.D., Antonellis, K.J., Scherf, U., et al., 2003. Exploration, normalization, and summaries of high density oligonucleotide array probe level data. Biostatistics 4(2): 249-64.

[33] Bolstad, B.M., Irizarry, R.A., Astrand, M., Speed, T.P., 2003. A comparison of normalization methods for high density oligonucleotide array data based on variance and bias. Bioinformatics 19(2): 185-93.

[34] Dai, M., Wang, P., Boyd, A.D., Kostov, G., Athey, B., Jones, E.G., et al., 2005. Evolving gene/transcript definitions significantly alter the interpretation of GeneChip data. Nucleic Acids Research 33(20): 1-9.

[35] Piccolo, S.R., Withers, M.R., Francis, O.E., Bild, A.H., Johnson, W.E., 2013. Multiplatform single-sample estimates of transcriptional activation. Proceedings of the National Academy of Sciences of the United States of America 110(44): 1777883.

[36] Schwamb, J., Feldhaus, V., Baumann, M., Patz, M., Brodesser, S., Brinker, R., et al., 2012. B-cell receptor triggers drug sensitivity of primary CLL cells by controlling glucosylation of ceramides. Blood 120(19): 3978-85.

[37] Han, J., Kaufman, R.J., 2016. The role of ER stress in lipid metabolism and lipotoxicity. Journal of Lipid Research 57(8): 1329-38. 
[38] Bensellam, M., Maxwell, E.L., Chan, J.Y., Luzuriaga, J., West, P.K., Jonas, J.-C., et al., 2016. Hypoxia reduces ER-to-Golgi protein trafficking and increases cell death by inhibiting the adaptive unfolded protein response in mouse beta cells. Diabetologia: 1492-502.

[39] Chen, Y., Brandizzi, F., 2013. IRE1: ER stress sensor and cell fate executor. Trends in Cell Biology 23(11): 547-55.

[40] Gupta, S., Deepti, A., Deegan, S., Lisbona, F., Hetz, C., Samali, A., 2010. HSP72 protects cells from ER stress-induced apoptosis via enhancement of IRE1??-xbp1 signaling through a physical interaction. PLoS Biology 8(7).

[41] Fu, Z., Abou-Samra, A.B., Zhang, R., 2015. A lipasin/Angpt18 monoclonal antibody lowers mouse serum triglycerides involving increased postprandial activity of the cardiac lipoprotein lipase. Scientific Reports 5(October): 18502.

[42] Li, Y., Teng, C., 2014. Angiopoietin-like proteins 3, 4 and 8: regulating lipid metabolism and providing new hope for metabolic syndrome. Journal of Drug Targeting 22(8): 679-87.

[43] Zhang, R., 2016. The ANGPTL3-4-8 model, a molecular mechanism for triglyceride trafficking. Open Biology 6(4): 150272.

[44] Gusarova, V., Alexa, C. a., Wang, Y., Rafique, A., Kim, J.H., Buckler, D., et al., 2015. ANGPTL3 blockade with a human monoclonal antibody reduces plasma lipids in dyslipidemic mice and monkeys. Journal of Lipid Research 56(7): 1308-17.

[45] Wang, Y., Gusarova, V., Banfi, S., Gromada, J., Cohen, J.C., Hobbs, H.H., 2015. Inactivation of ANGPTL3 reduces hepatic VLDL-triglyceride secretion. Journal of Lipid Research 56(7): 1296-307.

[46] Desai, U., Lee, E.C., Chung, K., Gao, C., Gay, J., Key, B., et al., 2007. Lipidlowering effects of anti-angiopoietin-like 4 antibody recapitulate the lipid phenotype found in angiopoietin-like 4 knockout mice. Proc Natl Acad Sci U S A 104(28): 11766-71.

[47] Siri-Tarino, P.W., Sun, Q., Hu, F.B., Krauss, R.M., 2010. Saturated fatty acids and risk of coronary heart disease: Modulation by replacement nutrients. Current Atherosclerosis Reports 12(6): 384-90.

[48] Cao, J., Dai, D.-L., Yao, L., Yu, H.-H., Ning, B., Zhang, Q., et al., 2012. Saturated fatty acid induction of endoplasmic reticulum stress and apoptosis in human liver cells via the PERK/ATF4/CHOP signaling pathway. Molecular and Cellular Biochemistry 364(1-2): 115-29.

[49] Egnatchik, R.A., Leamy, A.K., Noguchi, Y., Shiota, M., Young, J.D., 2014. Palmitate-induced activation of mitochondrial metabolism promotes oxidative stress and apoptosis in H4IIEC3 rat hepatocytes. Metabolism: Clinical and Experimental 63(2): 283-95.

[50] Schilling, J.D., Machkovech, H.M., He, L., Sidhu, R., Fujiwara, H., Weber, K., et al., 2013. Palmitate and lipopolysaccharide trigger synergistic ceramide production 
in primary macrophages. Journal of Biological Chemistry 288(5): 2923-32.

[51] Leamy, A.K., Hasenour, C.M., Egnatchik, R.A., Trenary, I.A., Yao, C., Patti, G.J., et al., 2016. Knockdown of Triglyceride Synthesis Does Not Enhance Palmitate Lipotoxicity or Prevent Oleate-Mediated Rescue in Rat Hepatocytes. Biochimica et Biophysica Acta (BBA) - Molecular and Cell Biology of Lipids 1861(9): 1005-14.

[52] Robblee, M.M., Kim, C.C., Abate, J.P., Valdearcos, M., Sandlund, K.L.M., Shenoy, M.K., et al., 2016. Saturated Fatty Acids Engage an IRE1 $\alpha$-Dependent Pathway to Activate the NLRP3 Inflammasome in Myeloid Cells. Cell Reports: 1-13, Doi.

[53] Dumas, J.A., Bunn, J.Y., Nickerson, J., Crain, K.I., Ebenstein, D.B., Tarleton, E.K., et al., 2016. Dietary saturated fat and monounsaturated fat have reversible effects on brain function and the secretion of pro-inflammatory cytokines in young women. Metabolism 65(10): 1582-8.

[54] L'homme, L., Esser, N., Riva, L., Scheen, A., Paquot, N., Piette, J., et al., 2013. Unsaturated fatty acids prevent activation of NLRP3 inflammasome in human monocytes/macrophages. Journal of Lipid Research 54: 2998-3008.

[55] Kennedy, A., Martinez, K., Chuang, C.-C., LaPoint, K., McIntosh, M., 2009. Saturated fatty acid-mediated inflammation and insulin resistance in adipose tissue: mechanisms of action and implications. The Journal of Nutrition 139(1): 1-4.

[56] Borradaile, N.M., Han, X., Harp, J.D., Gale, S.E., Ory, D.S., Schaffer, J.E., 2006. Disruption of endoplasmic reticulum structure and integrity in lipotoxic cell death. Journal of Lipid Research 47(12): 2726-37.

[57] Leamy, A.K., Egnatchik, R. a., Shiota, M., Ivanova, P.T., Myers, D.S., Brown, H.A., et al., 2014. Enhanced synthesis of saturated phospholipids is associated with ER stress and lipotoxicity in palmitate treated hepatic cells. Journal of Lipid Research 55(7): 1478-88.

[58] Volmer, R., van der Ploeg, K., Ron, D., 2013. Membrane lipid saturation activates endoplasmic reticulum unfolded protein response transducers through their transmembrane domains. Proceedings of the National Academy of Sciences of the United States of America 110(12): 4628-33.

[59] Kitai, Y., Ariyama, H., Kono, N., Oikawa, D., Iwawaki, T., Arai, H., 2013. Membrane lipid saturation activates IRE1?? without inducing clustering. Genes to Cells 18(9): 798-809.

[60] Deguil, J., Pineau, L., Rowland Snyder, E.C., Dupont, S., Beney, L., Gil, A., et al., 2011. Modulation of Lipid-Induced ER Stress by Fatty Acid Shape. Traffic 12(3): 349-62.

[61] Dhayal, S., Welters, H.J., Morgan, N.G., 2008. Structural requirements for the cytoprotective actions of mono-unsaturated fatty acids in the pancreatic beta-cell line, BRIN-BD11. British Journal of Pharmacology 153(8): 1718-27.

[62] Hamada, Y., Nagasaki, H., Fujiya, A., Seino, Y., Shang, Q.L., Suzuki, T., et al., 2014. Involvement of de novo ceramide synthesis in pro-inflammatory adipokine 
secretion and adipocyte-macrophage interaction. Journal of Nutritional Biochemistry 25(12): 1309-16.

[63] Anderson, E.K., Hill, A.A., Hasty, A.H., 2012. Stearic acid accumulation in macrophages induces toll-like receptor 4/2-independent inflammation leading to endoplasmic reticulum stress-mediated apoptosis. Arteriosclerosis, Thrombosis, and Vascular Biology 32(7): 1687-95.

[64] Jinjuvadia, R., Antaki, F., Lohia, P., Liangpunsakul, S., 2017. The association between nonalcoholic fatty liver disease and metabolic abnormalities in the United States Population. Journal of Clinical Gastroenterology 51(2): 160-6. 


\section{Supplemental Material}

\section{Supplemental Table 1: Composition of test diets}

Ingredients (\%)

Cocoa Butter

Canola Oil

Palm Olein Oil

Hydrogenated Soy Oil 0

Casein- Vitamin Tested 23.3060

Sucrose

19.7090

Maltodextrin

Dextrin

8.4830

5.8270

2.9200

1.9230

1.9230

13.2265

7.8536

0

23.3059

19.7090

11.6530

8.4830

5.8270

2.9200

2.9200

Potassium Citrate,

Tribasic Monohydrate

Calcium Phosphate

1.5150

1.5150

1.5150

AIN-76A Vitamin Mix 1.1650

1.1650

1.1650

DIO Mineral Mix

1.1650

1.1650

1.1650

Calcium Carbonate

0.6410

0.6410

0.6410

L-Cystine

0.3500

0.3500

0.3500

Choline Bitartate

0.2330

0.2330

0.0300

0.0300

(orange) 


\section{Supplemental Table 2: Primer sequences of genes}

\begin{tabular}{|c|c|c|}
\hline Genes & Forward primer & Reverse primer \\
\hline m36B4 & ATGGGTACAAGCGCGTCCTG & GCCTTGACCTTTTCAGTAAG \\
\hline mGrp78 & AGGACAAGAAGGAGGATGTGGG & ACCGAAGGGTCATTCCAAGTG \\
\hline mGrp94 & AAACGGCAACACTTCGGTCAG & GCATCCATCTCTТСТСССТСАТС \\
\hline $\mathrm{mXbp} 1$ & GCAGCAAGTGGTGGATTTGG & AGATGTTCTGGGGAGGTGACAAC \\
\hline $\mathrm{mXbp} 1 \mathrm{~s}$ & GAGTCCGCAGCAGGTG & GTGTCAGAGTCCATGGGA \\
\hline mDdit3 & CTGGAAGCCTGGTATGAGGAT & CAGGGTCAAGAGTAGTGAAGGT \\
\hline mDdit4 & CAAGGCAAGAGCTGCCATAG & CCGGTACTTAGCGTCAGGG \\
\hline mGdf15 & CTGGCAATGCCTGAACAACG & GGTCGGGACTTGGTTCTGAG \\
\hline $\mathrm{mCxcl} 2$ & CCAACCACCAGGCTACAGG & GCGTCACACTCAAGCTCTG \\
\hline mPtgs 2 & TTCAACACACTCTATCACTGGC & AGAAGCGTTTGCGGTACTCAT \\
\hline mErn1 & ACACCGACCACCGTATCTCA & CTCAGGATAATGGTAGCCATGTC \\
\hline mHerpud & d1 CCTGGCTTCTCTGGCTACAC & GTCGGGACAAAAGTTCCTGA \\
\hline $\mathrm{mHp} 2$ & GCTATGTGGAGCACTTGGTTC & CACCCATTGCTTCTCGTCGTT \\
\hline $\mathrm{mLcn} 2$ & TGGAAGAACCAAGGAGCTGT & GGTGGGGACAGAGAAGATGA \\
\hline mSAA2 & GCGAGCCTACACTGACATGA & TTTTCTCAGCAGCCCAGACT \\
\hline $\mathrm{mTNFa}$ & CTTCCATCCAGTTGCCTTCTTG & AATTAAGCCTCCGACTTGTGAAG \\
\hline mIL6 & CAACCTCCTCTCTGCCGTCAA & TGACTCCAAAGTAGACCTGCCC \\
\hline $\mathrm{mCD} 68$ & CCAATTCAGGGTGGAAGAAA & CTCGGGCTCTGATGTAGGTC \\
\hline
\end{tabular}




\section{Supplemental Table 3: Detailed fatty acid profile of diets}

Fatty acids $\quad$ Saturated (\%) $\quad$ Cis-Unsaturated (\%) $\quad$ Trans-Unsaturated (\%)

\begin{tabular}{|c|c|c|c|}
\hline C $14: 0$ & 0.18 & 0.44 & 0.14 \\
\hline C $14: 19 t$ & 0 & 0 & 0 \\
\hline C 14:19c & 0 & 0 & 0 \\
\hline C 16:0 & 23.76 & 16.16 & 11.01 \\
\hline C 16:19t & 0 & 0 & 0 \\
\hline C $16: 19 \mathrm{c}$ & 0.18 & 0.26 & 0 \\
\hline C 17:0 & 0.28 & 0 & 0.17 \\
\hline C $17: 110 \mathrm{c}$ & 0 & 0 & 0 \\
\hline C 18:0 & 31.59 & 2.99 & 8.23 \\
\hline C $18: 17+8 t$ & 0.11 & 0 & 4.28 \\
\hline C $18: 19 t$ & 0 & 0.11 & 3.33 \\
\hline C $18: 111 \mathrm{t}$ & 0 & 0 & 6.66 \\
\hline C $18: 15 c+7 c+12 t$ & 0 & 0 & 5.03 \\
\hline C $18: 19 c+13 t$ & 30.98 & 49.24 & 23.84 \\
\hline C $18: 111 c+15 t$ & 0.87 & 2.54 & 2.05 \\
\hline C $18: 112 c$ & 0 & 0.23 & 6.85 \\
\hline C $18: 113 c$ & 0 & 0.10 & 0.91 \\
\hline C $18: 115 c$ & 0 & 0 & 0.65 \\
\hline C $18: 2 \mathrm{tt}$ & 0 & 0 & 0.78 \\
\hline C $18: 2 \mathrm{ct}$ & 0 & 0.19 & 0 \\
\hline C $18: 2$ tc & 0 & 0.11 & 0 \\
\hline C $18: 2 \mathrm{cc}$ & 9.67 & 19.96 & 7.56 \\
\hline C $18: 3 \mathrm{ttt}$ & 0 & 0 & 0 \\
\hline C 20:0 & 1.10 & 0.55 & 0.31 \\
\hline C $18: 3 \mathrm{tct}$ & 0 & 0 & 0 \\
\hline C $18: 3 \mathrm{ctt}$ & 0 & 0 & 0 \\
\hline C $18: 3 \mathrm{ctc}$ & 0 & 0.09 & 0 \\
\hline C $18: 3$ tcc & 0 & 0.19 & 0 \\
\hline C $18: 3 \mathrm{ccc}$ & 1.16 & 5.03 & 1.00 \\
\hline C $20: 111 \mathrm{c}$ & 0 & 0.84 & 0.21 \\
\hline Unspecified cis/trans & 0 & 0 & 11.01 \\
\hline Unknown fatty acids & 0.14 & 1.04 & 6.03 \\
\hline
\end{tabular}


Supplemental Table 4: Overview of clinical observations in Angptl4-/- mice fed the different diets

Touton giant cells

Enlarged lymp nodes

SAA elevated

Chylous ascites

Weight loss
Saturated fat

Yes (6/7)

Yes, massive

Yes

Yes

Yes
Unsaturated fat

No $(0 / 5)$

Yes, slightly

No

No

No
Trans fat

Yes (9/13)

Yes, slightly

No

No

No 
Chapter 5 
Angptl4-hypomorphic mice expressing truncated $\mathrm{N}$-terminal ANGPTL4 exhibit lymphadenopathy and mild chylous ascites upon high fat feeding

Antwi-Boasiako Oteng*, Philip M. M. Ruppert*, Lily Boutens, Wieneke Dijk, Gunilla Olivecrona, Rinke Stienstra, Sander Kersten

* Authors contributed equally 


\begin{abstract}
Background \& Aim: ANGPTL4 regulates plasma lipids, making it an attractive target for correcting dyslipidemia. However, ANGPTL4 inactivation in mice fed a high fat diet causes chylous ascites, an acute phase response, and mesenteric lymphadenopathy. Here, we investigated the role of ANGPTL4 in the abovementioned pathologies using Angptl4 hypomorphic mice. In addition, we studied the influence of ANGPTL4 on lipid uptake in macrophages.
\end{abstract}

Results: Angptl4 expression in peritoneal and bone marrow-derived macrophages was highly induced by lipids. Recombinant ANGPTL4 decreased lipid uptake in macrophages, whereas deficiency of ANGPTL4 increased lipid uptake and enhanced lipid-induced genes. Hypomorphic Angptl4 mice with partial expression of N-terminal ANGPTL4 exhibited reduced fasting plasma triglyceride, cholesterol, and non-esterified fatty acid levels, strongly resembling Angptl $4^{-/-}$mice. However, during high fat feeding, hypomorphic Angptl4 mice showed markedly delayed and attenuated elevation in plasma serum amyloid A and much milder chylous ascites than Angptl $^{-/-}$mice, despite similar abundance of lipid-laden giant cells in mesenteric lymph nodes.

Conclusion: ANGPTL4 deficiency increases lipid uptake into macrophages. Compared with the absence of ANGPTL4, low levels of N-terminal ANGPTL4 are sufficient to mitigate the development of chylous ascites and an acute phase response in mice, whereas mesenteric lymphadenopathy is not affected. 


\section{Introduction}

Elevated plasma triglyceride levels are increasingly considered as an independent risk factor for cardiovascular diseases [1-3]. Triglycerides circulate in the blood in two major forms: as chylomicrons carrying the dietary triglycerides, and as very-low density lipoproteins carrying endogenously produced triglycerides [3,4]. The clearance of plasma triglycerides is primarily mediated by the action of lipoprotein lipase (LPL). This secretory enzyme is produced in parenchymal cells of fat tissue, skeletal muscle, and heart, as well as by macrophages. With the help of the endothelial protein glycosylphosphatidylinositol-anchored high-density lipoprotein binding protein 1 (GPIHBP1), LPL is transferred from the surface of the sub-endothelial myocytes and adipocytes to the luminal side of the capillary endothelium. There, LPL hydrolyses the triglycerides contained in the triglyceride-rich lipoproteins to release fatty acids for uptake by the underlying tissues [5-9]. The activity of LPL is regulated posttranslationally by numerous factors, many of which are produced in the liver, including several apolipoproteins. In addition, LPL activity is governed by several members of the family of Angiopoietin-like proteins: ANGPTL3, ANGPTL4, and ANGPTL8 [5,10-12].

ANGPTL3 is produced in the liver and cooperates with ANGPTL8 to inhibit LPL activity in peripheral tissues. The role of ANGPTL3/ANGPTL8 in LPL regulation is particularly important in the fed state [13]. By contrast, ANGPTL4 mainly plays a role in LPL regulation in the fasted state. Individuals who carry an inactive variant of ANGPTL4 exhibit lower levels of circulating triglycerides and have decreased odds of developing coronary heart disease [14,15]. In mice, overexpression of ANGPTL4 potently represses LPL activity and leads to hypertriglyceridemia, whereas deletion of ANGPTL4 stimulates LPL activity and drastically reduces plasma triglyceride levels $[16,17]$. In contrast to ANGPTL3, which functions as an endocrine factor, ANGPTL4 likely serves as a local regulator of LPL in tissues where LPL and ANGPTL4 are coproduced, in particular in adipose tissue [18]. Inhibition of LPL is mediated by the Nterminal coiled-coiled domain of ANGPTL4, leading to unfolding of LPL and conversion of the active LPL dimers into inactive monomers [19-21]. In adipocytes, ANGPTL4 promotes the cleavage and subsequent degradation of LPL, thereby preventing the delivery of LPL to the endothelial surface $[22,23]$. The important role of ANGPTL4 in governing plasma lipid levels in humans has made ANGPTL4 an attractive therapeutic target for correcting dyslipidemia and associated cardiovascular disorders.

However, we and others showed that disabling ANGPTL4, either via monoclonal antibody-mediated or genetic inactivation, leads to a highly pro-inflammatory and 
ultimately lethal phenotype in mice fed a high saturated fat diet. This marked phenotype includes mesenteric lymphadenopathy, characterized by the presence of lipid-laden Touton giant cells in mesenteric lymph nodes, as well as fibrinopurulent peritonitis, chylous ascites, and marked elevation of plasma levels of acute phase proteins, such as serum amyloid A and haptoglobin [24,25]. Accumulation of lipids in mesenteric lymph nodes was also observed in several female monkeys treated with an anti-ANGPTL4 antibody [15]. Although there is so far no evidence pointing to the occurrence of abdominal lymphadenopathy in humans homozygous for an inactive ANGPTL4 variant, currently the therapeutic prospects of whole body ANGPLT4 inactivation are not very favourable.

The mouse model that revealed the effect of ANGPTL4 inactivation on lymphadenopathy and other pathologies is a whole body ANGPTL4 knock-out model caused by the deletion of exons 2 and 3 and part of intron 1, leading to the absence of ANGPTL4 protein [26]. Whether other and milder forms of genetic ANGPTL4 inactivation in mice provoke a similar phenotype upon feeding a high saturated fat diet is unclear. According, the first aim of our paper was to further investigate the role of ANGPTL4 in the aforementioned pathologies using Angptl4 hypomorphic mice.

The deleterious effects of ANGPTL4 inactivation observed in mesenteric lymph nodes were previously attributed to the role of ANGPTL4 as LPL inhibitor in mesenteric lymph node macrophages, thereby preventing excessive fatty acid uptake and a concomitant pro-inflammatory response. It was shown that recombinant ANGPTL4 is able to limit lipid uptake into peritoneal macrophages incubated with chyle [24]. However, whether endogenous ANGPTL4 suppresses lipid uptake into macrophages was not addressed. Accordingly, the second aim of our paper was to examine the influence of endogenous ANGPTL4 on lipid uptake in macrophages. 


\section{Materials and Methods}

\section{Animal studies}

Animal studies were performed in male purebred wild-type (WT), Angptl4 ${ }^{\text {hyp }}$ and Angptl4 $^{-/}$mice on a C57Bl/6 background. The Angptl4 ${ }^{\text {hyp }}$ mice were a kind donation from Dr. Nguan Soon Tan (Nanyang Technological University, Singapore). These mice can also be classified as Angptl4 knockout-first mice [27], which upon stepwise crossing with mice expressing flippase recombinase and Cre recombinase can be used to generate a tissue-specific Angptl4 knockout mice model.

High fat diet intervention: Eleven to 16-week-old mice (10 wild-type, 11 Angptl4 $^{\text {hyp }}$ and 8 Angptl4 $\%$ ) were fed a high fat diet that provided $45 \%$ energy percent as triglycerides (D12451, Research Diets Inc., New Brunswick, NJ). After week 2 and 4 of the intervention, blood samples were collected to measure plasma serum amyloid A (SAA). After 20 weeks, blood was collected via orbital puncture under isoflurane anaesthesia. Immediately thereafter, the mice were euthanized by cervical dislocation. Tissues were excised and immediately frozen in liquid nitrogen followed by storage at $-80^{\circ} \mathrm{C}$. The animal studies were approved by the Local Animal Ethics Committee of Wageningen University (AVD104002015236: 2016.W-0093.005, 2016.W-0093.007).

Fasting intervention: Mice were fed a standard chow diet since weaning. Mice were either kept on chow or fasted for 24 hours and euthanized between 9.00 and 11.00 hours. Blood was collected via orbital puncture under isoflurane anaesthesia. Immediately thereafter, the mice were euthanized by cervical dislocation. Tissues were excised and immediately frozen in liquid nitrogen followed by storage at $-80^{\circ} \mathrm{C}$.

Intraperitoneal glucose tolerance test: After 18 weeks of high fat diet, the mice were fasted for 5 hours prior to the glucose tolerance test. The mice were injected intraperitoneally with glucose (1 $\mathrm{g} / \mathrm{kg}$ body weight) (Baxter, Deerfield, IL, USA). Blood samples from tail vein bleeding were tested for glucose levels at different time points following glucose injection using a GLUCOFIX Tech glucometer and glucose sensor test strips (GLUCOFIX Tech, Menarini Diagnostics, Valkenswaard, The Netherlands).

\section{Histology}

Haematoxylin and eosin staining was performed on the mesenteric lymph nodes. During the mice sections, lymph nodes were isolated into plastic cassettes and immediately fixated in 4\% paraformaldehyde. The tissues were processed and embedded into paraffin blocks. Thin sections of the blocks were made at $5 \mu \mathrm{M}$ using a microtome and the placed 
onto Superfrost glass slides followed by overnight incubation at $37^{\circ} \mathrm{C}$. The tissues were stained in Mayer hematoxylin solution for 10 minutes and in eosin for 10 seconds at room temperature with intermediate washings in ethanol. The tissues were allowed to dry at room temperature followed by imaging using light microscope.

\section{Quantification of plasma parameters}

Blood samples were collected into EDTA-coated tubes and centrifuged at $4^{\circ} \mathrm{C}$ for 15 min at $10,000 \mathrm{~g}$. Plasma was collected and stored at $-80^{\circ} \mathrm{C}$. ELISA kits were used to measure plasma serum amyloid (SAA) (Tridelta Development Ltd, Ireland) and haptoglobin (Abcam, Cmabridge, UK) according to manufacturer's protocol. Measurement of plasma levels of triglycerides, non-esterified fatty acids (NEFA) and glycerol were performed using kits from HUMAN Diagnostics (Wiesbaden, Germany) according to manufacturer's protocol. Plasma levels of cholesterol and glucose were also measured using kits from Diasys Diagnostics Systems (Holzheim, Germany) according to manufacturer's protocol.

\section{LPL activity measurements}

LPL activity levels in epididymal white adipose tissues were measured in triplicate with a $[3 \mathrm{H}]$ triolein-labeled lipid emulsion as previously described [28]. Protein contents in homogenates of adipose tissue were measured using Markwell's modified Lowry method [29].

\section{Cell Culture}

Bone marrow cells were isolated from femurs of wild-type or Angptl $4^{-/}$mice following standard protocol and differentiated into macrophages (bone-marrow derived macrophages) during 6-8 days culturing in DMEM containing 10\% FBS and 1\% PS supplemented with 10\% L929-conditioned medium (L929). After 6-8 days, nonadherent cells were removed and adherent cells were washed and plated in 6-, 12-, or 48 well plates in DMEM/FCS/PS + L929. After 24 hours, the cells were washed with PBS and treated. Peritoneal macrophages were obtained by infusion, and subsequent collection of ice-cold PBS from the abdominal cavity, and frozen directly or brought into culture in DMEM containing 10\% FBS and 1\% PS. RAW264.7 macrophages were cultured in DMEM containing 10\% FBS and 1\% P/S. All cells were maintained in a humidified incubator at $37^{\circ} \mathrm{C}$ with $5 \% \mathrm{CO}_{2}$.

Inguinal white adipose tissue from 3-4 WT, Angptl4 ${ }^{\text {hyp }}$ and Angptl4 ${ }^{-/-}$mice was collected and placed in Dulbecco's modified eagle's medium (DMEM; Lonza, Verviers, Belgium) supplemented with $1 \%$ Penicillin/Streptomycin (PS) and 1\% BSA (Sigma-Aldrich). 
Material was minced with scissors and digested in collagenase-containing medium (DMEM with $3.2 \mathrm{~mm} \mathrm{CaCl} 2,1.5 \mathrm{mg} / \mathrm{ml}$ collagenase type II (C6885, Sigma-Aldrich), $10 \%$ foetal bovine serum (FBS), $0.5 \%$ BSA, and $15 \mathrm{~mm}$ HEPES) for $1 \mathrm{~h}$ at $37{ }^{\circ} \mathrm{C}$, with occasional vortexing. Cells were filtered through a $100-\mu \mathrm{m}$ cell strainer (Falcon) to remove remaining cell clumps and lymph nodes. The cell suspension subsequently was centrifuged at $1600 \mathrm{rpm}$ for $10 \mathrm{~min}$ and the pellet was resuspended in erythrocyte lysis buffer (155 mm NH $4 \mathrm{Cl}, 12 \mathrm{~mm} \mathrm{NaHCO}, 0.1 \mathrm{~mm}$ EDTA). Upon incubation for $2 \mathrm{~min}$ at room temperature, cells were centrifuged at $1200 \mathrm{rpm}$ for $5 \mathrm{~min}$ and the pelleted cells were resuspended in DMEM $+10 \%$ FCS $+1 \% \mathrm{P} / \mathrm{S}$ and plated. Upon confluence, the cells were differentiated according to the protocol as described previously $(14,15)$. Briefly, confluent SVFs were plated in 1:1 surface ratio, differentiation was induced 2 days afterwards by switching to an differentiation induction cocktail (DMEM containing $10 \% \mathrm{FBS}, 1 \% \mathrm{P} / \mathrm{S}, 0.5 \mathrm{~mm}$ isobutylmethylxanthine, $1 \mu \mathrm{M}$ dexamethasone, $7 \mu \mathrm{g} / \mathrm{ml}$ insulin and $1 \mu \mathrm{M}$ rosiglitazone) for 3 days. Subsequently, cells were maintained in DMEM supplemented with $10 \% \mathrm{FBS}, 1 \% \mathrm{P} / \mathrm{S}$, and $7 \mu \mathrm{g} / \mathrm{ml}$ insulin for 3-6 days and switched to DMEM with $10 \% \mathrm{FBS}$ and $1 \% \mathrm{P} / \mathrm{S}$ for 3 days. Average rate of differentiation was at least $80 \%$ as determined by eye.

\section{Cell culture experiments and chemical treatments}

Bone marrow-derived macrophages and peritoneal macrophages were exposed to $1 \mathrm{mM}$ intralipid or $500 \mu \mathrm{M}$ oleic acid (Sigma-Aldrich) for 6 hours in DMEM/PS media. The oleic acid was conjugated to BSA at a ratio of about 2:1 (BSA:oleic acid). Bone-marrow derived macrophages were also treated with synthetic PPAR agonists; $1 \mu \mathrm{M}$ Wy14643, $1 \mu \mathrm{M}$ rosiglitazone, $1 \mu \mathrm{M} \mathrm{L165041,} 1 \mu \mathrm{M}$ GW501516 or vehicle control for 6 hours. All PPAR agonists were obtained from Sigma-Aldrich. Peritoneal macrophages and RAW264.7 cells were incubated for 6 hours with lymph (final triglyceride concentration of $2 \mathrm{mM}$, which was collected from rats provided with palm-oil based high fay diet overnight). Bone-marrow derived macrophages were co-incubated for 6 hours with 10 $\mu \mathrm{M}$ orlistat (Sigma-Aldrich) and $1 \mathrm{mM}$ intralipid. RAW264.7 macrophages treated with $1 \mathrm{mM}$ intralipid or lymph was incubated with or without $0.5 \mu \mathrm{g} / \mathrm{mL}$ recombinant ANGPTL4 (R\&D Systems, Abingdon, UK).

\section{Oil red-O staining}

Oil red-O staining was performed in RAW264.7 cells incubated with intralipid or lymph in the presence or absence of recombinant ANGPTL4. A stock of oil red O (Sigma) was prepared by dissolving $0.5 \mathrm{~g}$ in $500 \mathrm{ml}$ of isopropanol. Working concentrations were made by dissolving stock concentrations with water ( 3 stock: 2 water) and filtered. 
Treated cells were washed twice with PBS, followed by fixation with $4 \%$ formalin for $30 \mathrm{~min}$. The cells were then washed twice with PBS and incubated with oil red-O dye for $20 \mathrm{~min}$. The stained cells were washed 3 times with $\mathrm{ddH}_{2} \mathrm{O}$ after which cells were visualised under a light microscope and pictures taken.

\section{Bodipy staining}

For visualization of lipid droplets, treated bone marrow-derived macrophages of wildtype and $\mathrm{Angptl}^{-/ /}$mice were washed with PBS, fixated in $4 \%$ formalin and subsequently stained with $1 \mathrm{ng} / \mathrm{mL}$ bodipy (ThermoFisher Scientific, Landsmeer, The Netherlands).

\section{RNA isolation and quantitative real-time PCR}

Total RNA was isolated from tissues and cells by homogenizing in TRIzol (Thermo Fisher Scientific) either with a QIagen Tissue Lyser II (Qiagen, Venlo, The Netherlands) or by pipetting up and down. Reverse transcription was performed using the iScript ${ }^{\mathrm{TM}}$ cDNA Synthesis Kit (Biorad) according to the manufacturer's protocol. Quantitative PCR amplifications were done on a CFX384 real-time PCR platform (Bio-Rad) with the SensiMix PCR mix from Bioline (GC Biotech, Alphen aan de Rijn, The Netherlands). Primer sequences of genes are provided in Supplemental Table 1. Expression of target genes was normalized to $36 \mathrm{~b} 4$.

\section{Extracellular flux analysis}

Real-time oxygen consumption (OCR) and extracellular acidification rates (ECAR) of bone marrow-derived macrophages from wild-type and Angptl4 ${ }^{-/ 2}$ mice were assessed using XF-96 Extracellular Flux Analyzer (Seahorse, Bioscience, Santa Clara, CA, USA). Basal metabolic rates of bone marrow-derived macrophages seeded in quintuplicate were determined during three consecutive measurements in unbuffered Seahorse medium ( 8.3 g DMEM powder, $0.016 \mathrm{~g}$ phenol red and $1.85 \mathrm{~g} \mathrm{NaCl}$ in $1 \mathrm{~L}$ milli-Q, $\mathrm{pH} 7.4$ at $37^{\circ} \mathrm{C}$, sterile-filtered) conatining $25 \mathrm{mM}$ glucose and $2 \mathrm{mM} \mathrm{L}$-glutamine. After basal measurements, three consecutive measurements were made following addition of 1.5 $\mu \mathrm{M}$ oligomycin, $1.5 \mathrm{mM}$ carbonyl cyanide-4-(trifluoromethoxy)phenylhydrazone (FCCP) and combination of $2 \mu \mathrm{M}$ antimycin and $1 \mu \mathrm{M}$ rotenone. Pyruvate $(1 \mathrm{mM})$ was added together with FCCP to fuel maximal respiration. All compounds used during the Seahorse runs were acquired from Merck. Signals were normalized to relative DNA content in the wells using the Quanti-iT ${ }^{\mathrm{TM}} \mathrm{dsDNA}$ Assay kit (ThermoFisher).

\section{Western immunoblotting}


To isolate protein, mouse fat pads, differentiated primary adipocytes, primary macrophages and whole adipose tissues were lysed in RIPA Lysis and Extraction Buffer (25 mm Tris-HCl, pH 7.6, $150 \mathrm{~mm} \mathrm{NaCl}, 1 \%$ Nonidet P-40, and 0.1\% SDS; Thermo Fisher Scientific) supplemented with protease and phosphatase inhibitors (Roche Diagnostics, Almere, The Netherlands). Following homogenization, lysates were placed on ice for $30 \mathrm{~min}$ and centrifuged 2-3 times at $13,000 \mathrm{rpm}$ for $10 \mathrm{~min}$ at $4{ }^{\circ} \mathrm{C}$ to remove fat and cell debris. Concentration of protein lysates was determined by using a bicinchoninic acid assay (Thermo Fisher Scientific). For assessment of LPL release, bone marrow derrived macrophages were treated for 20 mins with $10 \mathrm{IU} / \mathrm{mL}$ heparin (\# 012866-08, LEO Pharma). For assessment of glycosylation of LPL, 2-30 $\mu \mathrm{g}$ of proteins was digested with endoglycosidase $\mathrm{H}$ (EndoH), (New England BioLabs) according to manufacturer's protocol. Protein lysates (10-30 $\mu \mathrm{g}$ of protein/lane) were loaded onto 8$16 \%$ or $10 \%$ Criterion gels (Bio-Rad, Veenendaal, The Netherlands). Next, proteins were transferred onto a polyvinylidene difluoride membrane using the Transblot Turbo System (Bio-Rad). Membranes were probed with a goat anti-mouse LPL antibody (28), a rabbit anti-mouse HSP90 antibody (\#4874S, Cell signaling), a rat anti-mouse ANGPTL4 antibody (Kairos 142-2, Adipogen) and a rabbit anti-mouse ANGPTL4 antibody (742) [30] at 1:5000 (mLPL), 1:2000 (HSP90), or 1:1000 (ANGPTL4) dilutions. Blocking and the incubation of primary and secondary antibodies were all done in TBS, pH 7.5, 0.1\% Tween 20 (TBS-T), and 5\% (w/v) skimmed milk at 1:5000. In between, membranes were washed in TBS-T. Quantification was performed with the ChemiDoc MP system (Bio-Rad) and Clarity ECL substrate (Bio-Rad). Equal loading of medium samples was verified with HSP90.

\section{Statistical Analysis}

Statistical analyses were performed using one-way ANOVA followed by Tukey HSD test. Data are presented as mean \pm SEM (animal studies) or mean \pm SD (cell culture studies). $\mathrm{P}<0.05$ was considered statistically significant. 


\section{Results}

\section{Angptl4 expression is sensitive to lipids and regulates lipid uptake by macrophages}

We previously suggested that enhanced LPL-mediated lipid uptake in mesenteric lymph node macrophages may be at the basis of the pro-inflammatory phenotype in Angptl $4^{-1-}$ mice fed a high fat diet. To better understand the role of endogenous ANGPTL4 in lipid uptake in macrophages, we first studied the regulation of Angptl4 expression by lipids in macrophages. Treatment of peritoneal macrophages with oleic acid markedly increased Angptl4 mRNA (Fig. 1A).
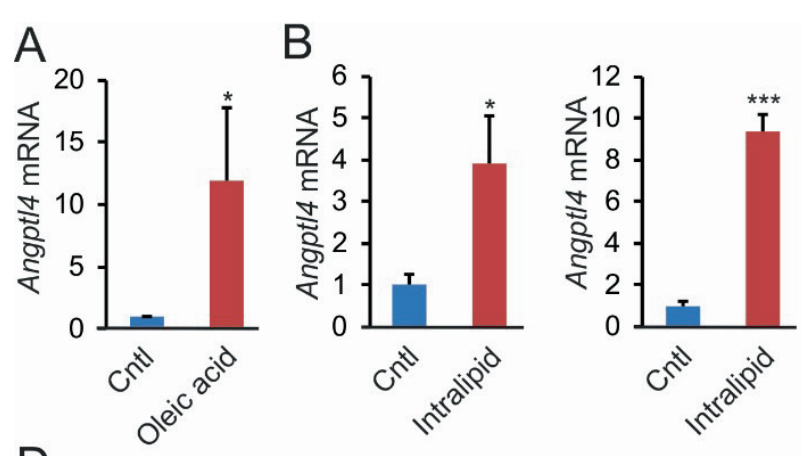

C
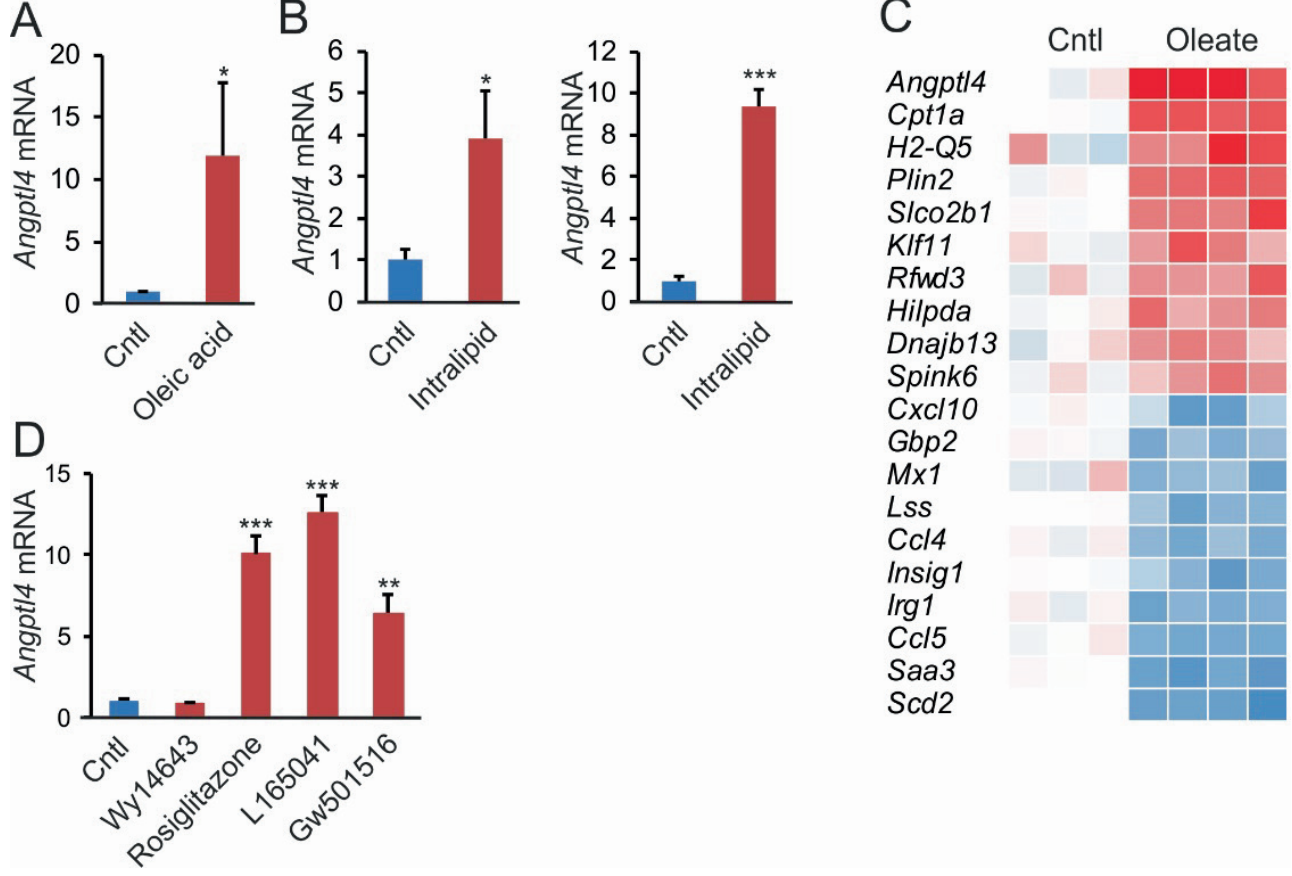

Figure 1. Angptl4 expression is induced in primary macrophages by lipid stimulation or PPAR agonists. (A) Angptl4 expression in peritoneal macrophages treated with $500 \mu \mathrm{M}$ oleic acid for 6 hours. (B) Angptl4 expression in peritoneal macrophages (left) and bone marrowderived macrophages (right) treated for 6 hours with $0.5 \mathrm{mM}$ intralipid. (C) Heatmap of top 10 most upregulated and top 10 most downregulated genes in bone marrow-derived macrophages treated for 5 hours with $250 \mathrm{uM}$ oleic acid or BSA (vehicle control) (GSE77104) [31]. (D) Angptl4 expression in bone marrow-derived macrophages treated for 6 hours with $1 \mu \mathrm{M}$ of PPAR agonists or control. mRNA expression was normalized to $36 b 4$. Data are mean $\pm \mathrm{SD} ;{ }^{*} \mathrm{p}<0.05,{ }^{* *} \mathrm{p}<0.01$ and $* * * \mathrm{p}<0.001$ relative to vehicle control. 
Similarly, treatment of peritoneal and bone-marrow derived macrophages with intralipid markedly induced Angptl4 mRNA (Fig. 1B). Interestingly, analysis of a publicly available transcriptomics dataset indicated that Angptl4 is the most highly induced gene in bone marrow-derived macrophages upon treatment with oleic acid (Fig. 1C) [31]. The effect of lipid treatment on Angptl4 expression is likely mediated by PPAR $\delta$ and/or PPAR $\gamma$, as Angptl4 is a well-established target of PPARs in other cell types and treatment with synthetic PPAR $\delta$ and PPAR $\gamma$ agonists caused a pronounced increase in Angptl4 mRNA (Fig. 1D).

To study the effect of exogenous ANGPTL4 on lipid uptake in macrophages, RAW264.7 macrophages were treated with intralipid in the presence or absence of recombinant ANGPTL4. ANGPTL4 clearly reduced lipid accumulation, as shown by decreased oil red $\mathrm{O}$ staining (Fig. 2A). Similar results were obtained in peritoneal macrophages incubated with lymph, an endogenous lipid source (Fig. 2B). Transcriptomics analysis of peritoneal macrophages treated with intralipid showed that the induction of lipidsensitive genes by intralipid was almost completely abolished in the presence of recombinant ANGPTL4 (Fig. 2C).

To study the effect of endogenous ANGPTL4 on lipid uptake in macrophages, wild-type and Angptl4 ${ }^{-/}$macrophages were treated with intralipid. Lipid accumulation was distinctly higher in Angptl4 $4^{--}$macrophages than in wild-type macrophages, as shown by Bodipy staining (Fig. 2D). This effect could be abolished by treatment with the LPL inhibitor orlistat (Fig. 2D). Consistent with enhanced lipid uptake in Angptl4 ${ }^{-1-}$ macrophages, the effect of intralipid on the expression of lipid-sensitive genes was much more pronounced in Angptl4 ${ }^{-/}$bone marrow-derived macrophages than in wild-type macrophages (Fig. 2E). Similar results were obtained in peritoneal wild-type and Angptl $4^{-/ /}$macrophages upon loading with lymph (Fig. 2F). Together, these data strongly suggest that ANGPTL4 deficiency leads to elevated lipid uptake in macrophages via regulation of LPL.

\section{ANGPTL4 deficiency has no effect on LPL protein and on fuel utilization in primary macrophages}

We previously showed in primary adipocytes that ANGPTL4 deficiency is paralleled by elevated LPL protein, indicating that ANGPTL4 regulates LPL protein levels [22]. To study the influence of ANGPTL4 on LPL protein in macrophages, we performed Western blot for LPL in bone marrow-derived macrophages from wild-type and Angptl4${ }^{\prime}$ mice, with and without intralipid treatment. 

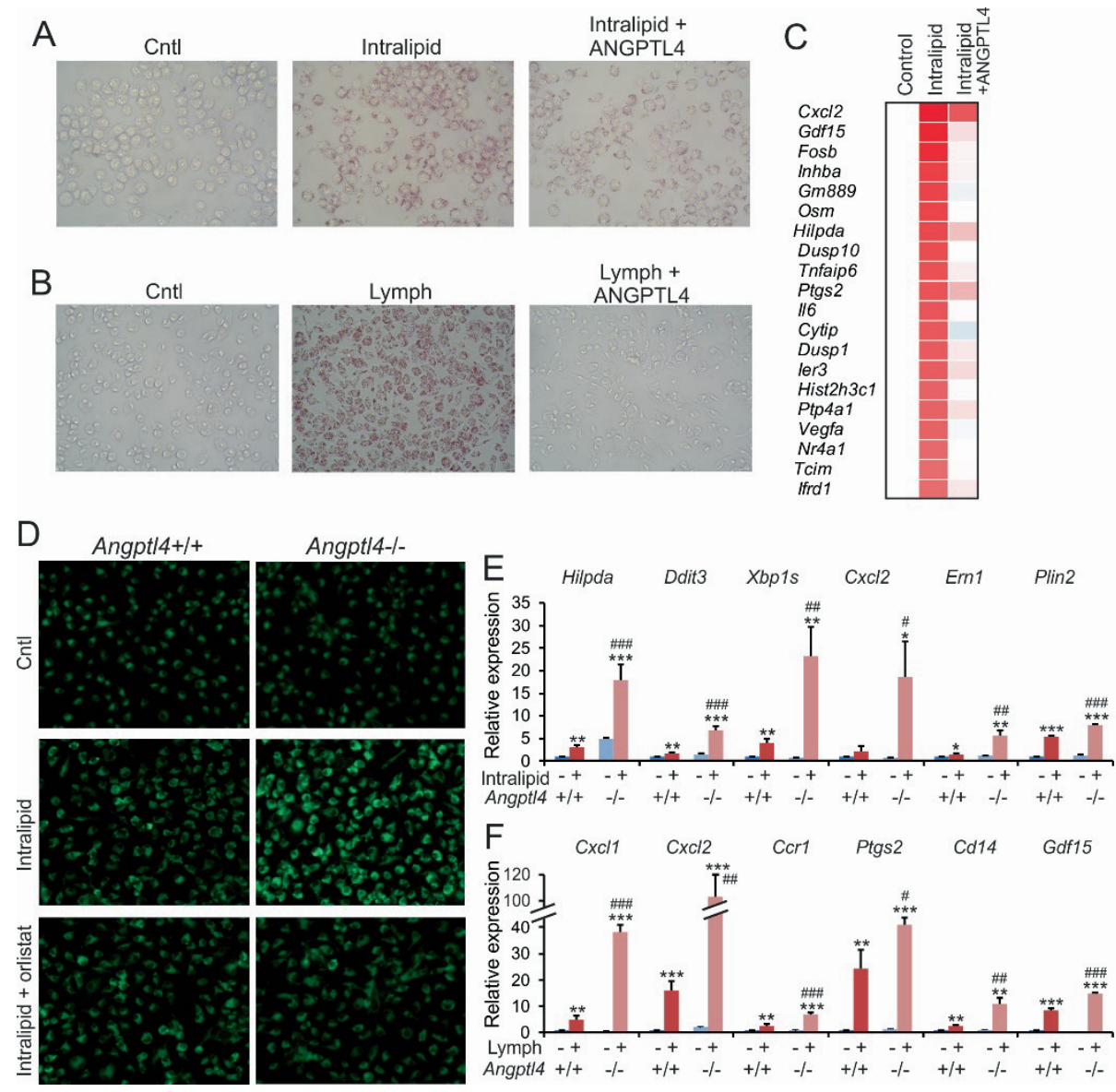

Figure 2. ANGPTL4 deficiency promotes macrophage lipid uptake and magnifies the induction of lipid-sensitive genes. (A) Oil red O staining of lipid droplets in RAW264.7 macrophages treated with $0.5 \mathrm{mM}$ intralipid for 6 hours in the presence or absence of recombinant ANGPTL4 $(0.5 \mu \mathrm{g} / \mathrm{mL})$. (B) Oil red O staining of lipid droplets in peritoneal macrophages treated for 6 hours with lymph (final triglyceride concentration of $2 \mathrm{mM}$ ) in the presence or absence of recombinant ANGPTL4 $(0.5 \mu \mathrm{g} / \mathrm{mL})$. (C) Heatmap showing expression profile of lipid-sensitive genes in peritoneal macrophages treated for 6 hours with $0.5 \mathrm{mM}$ intralipid in the presence or absence of recombinant ANGPTL4. (D) Bodipy staining of Angptl4 ${ }^{+/+}$and Angptl4 ${ }^{-/-}$bone marrow-derived macrophages cultured with regular culture medium (Cntl), or for 6 hours with 0.5 $\mathrm{mM}$ intralipid with or without orlistat . (E-F) mRNA expression of lipid sensitive genes in Angptl4 $^{+/+}$and Angptl4 ${ }^{-/}$bone marrow-derived macrophages treated for 6 hours with $1 \mathrm{mM}$ intralipid (E) or peritoneal macrophages treated for 6 hours with lymph (final triglyceride concentration of $2 \mathrm{mM}$ ) (F). mRNA expression was normalized to $36 b 4$. Data are mean $\pm \mathrm{SD} ;{ }^{*} \mathrm{p}$ $<0.05,{ }^{*} \mathrm{p}<0.01$ and ${ }^{* * *} \mathrm{p}<0.001$ relative to untreated wild-type; and $\# \mathrm{p}<0.05, \# \# \mathrm{p}<0.01$ and $\# \# \# \mathrm{p}<0.001$ relative to wild-type + intralipid/lymph. 
In contrast to what was observed in adipocytes, ANGPTL4 deficiency did not have any effect on LPL protein in macrophages and did not alter the ratio between the active, endonuclease H-resistant form and the inactive, endonuclease-sensitive form of LPL (Fig. 3A).
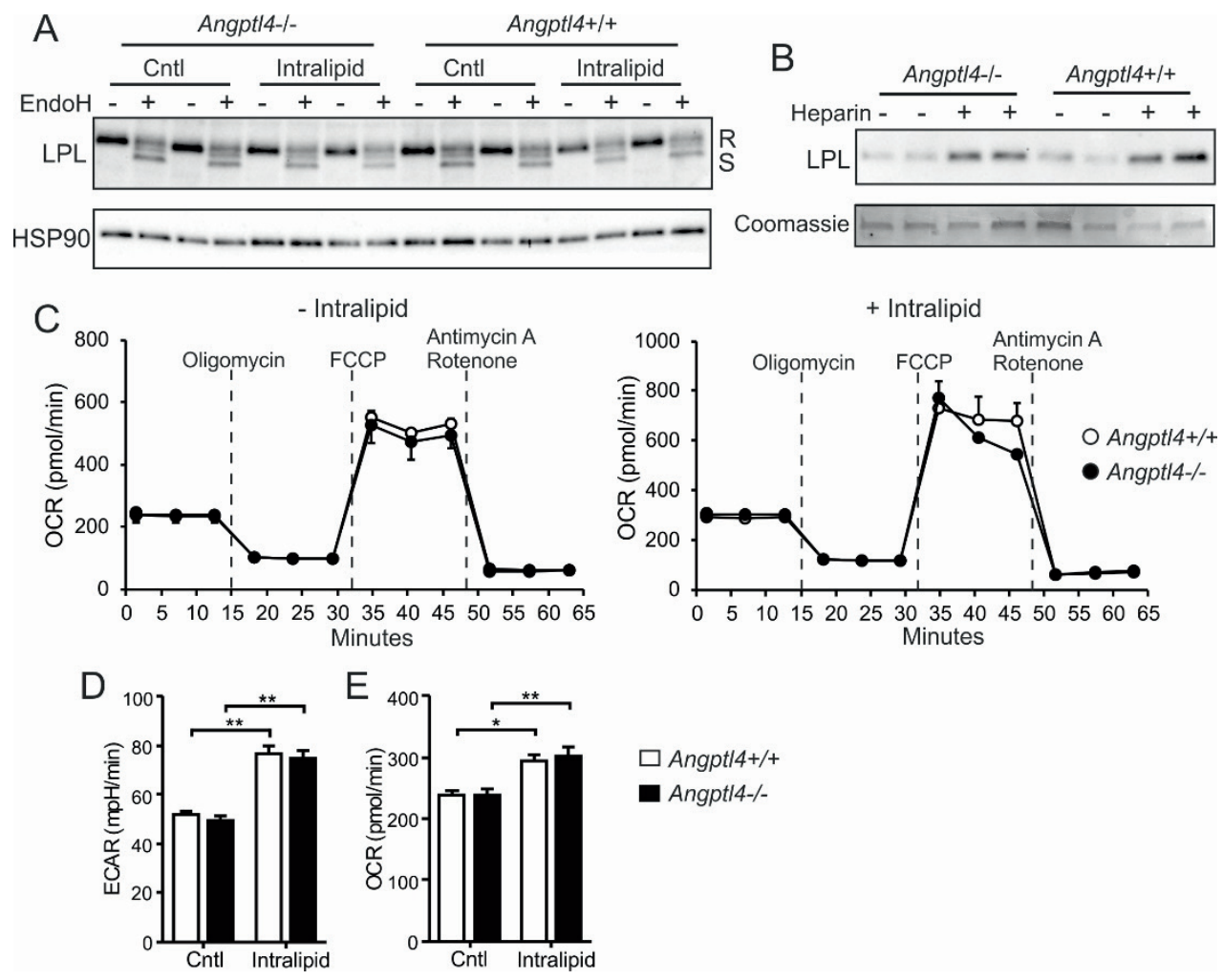

Figure 3. ANGPTL4 does not influence LPL processing and lipid oxidation in primary macrophages. (A) Immunoblot of LPL in Angptl4 ${ }^{+/+}$and Angptl4 $4^{-/-}$bone marrow-derived macrophages treated for 6 hours with or without $0.5 \mathrm{mM}$ intralipid in the presence or absence of endonuclease-H. (B) Immunoblot of LPL from culture medium of Angptl $^{+/+}$and $\mathrm{Angptl}^{-/-}$bone marrow-derived macrophages treated with or without heparin. HSP90 or Coomassie staining serve as loading control. (C) Real time changes in oxygen consumption rate (OCR) in Angptl4 ${ }^{+/+}$and Angptl $4^{-/}$bone marrow-derived macrophages treated with or without $0.5 \mathrm{mM}$ intralipid for 6 hours, assessed in unbuffered medium during sequential injections with Oligomycin, FCCP, and antimycin A + rotenone. (D) Basal extracellular acidification rate (ECAR) and oxygen consumption rate (OCR) of Angptl4 ${ }^{+/+}$and Angptl4 ${ }^{-/-}$bone marrow-derived macrophages incubated with or without $0.5 \mathrm{mM}$ intralipid for 6 hrours. Measurements were done in unbuffered medium. Data are mean $\pm \mathrm{SEM} ;{ }^{*} \mathrm{p}<0.05, * * \mathrm{p}<0.01$. 
In addition, the amount of LPL that could be released into the medium by treatment of the macrophages with heparin did not differ between the two genotypes (Fig. 3B). These data suggest that in macrophages, ANGPTL4 does not inhibit LPL activity by promoting LPL degradation.

To test if differences in lipid uptake are reflected in fuel utilization between the genotypes, we measured real time metabolic fluxes in bone marrow-derived macrophages using the Seahorse machine. No differences in basal and maximal oxidative phosphorylation were observed between wild-type and Angptl4 ${ }^{-1-}$ macrophages, both in the absence and in the presence of intralipid (Fig. 3C). Intralipid treatment increased glycolysis (ECAR) and oxidative phosphorylation (OCR) rates, which was not different between the wild-type and Angptl ${ }^{-/-}$macrophages (Fig. 3D). These data suggest that the inhibitory effect of ANGPTL4 on LPL-mediated lipid uptake in macrophages does not have any influence on fuel utilization.

\section{Angptl4-hypomorphic mice show partial expression of N-terminal exons and truncated ANGPTL4 protein in adipose tissue and primary adipocytes}

The second objective of this paper was to investigate whether in addition to the Angptl41- model, an alternative model of genetic Angptl4 inactivation also leads to a deleterious phenotype in mice fed a high saturated fat diet. To that end, we used Angptl4 knockout first mice. The Angptl4 knockout first allele contains a reading frame-independent LacZ gene trap cassette followed by a promoter-driven selection cassette in intron 3 (Fig. 4A). The Angptl4 knockout first allele was designed to create tissue-specific Angptl4 ${ }^{-/ 2}$ mice by subsequent crossing with mice expressing the FLP and Cre recombinase enzymes. Interestingly, based on sequence analysis, it can be predicted that when omitting the FLP and Cre recombinase reaction steps, the Angptl4 knockout first allele might lead to the production of a whole body mutant ANGPTL4 protein covering amino acid residues 1186, followed by 51 amino acids originating from the EN2a splice acceptor site, internal ribosome entry site, and part of LacZ (Fig. 4B). Accordingly, we hypothesized that the Angptl4 knockout first allele may give rise to a truncated ANGPTL4 protein that contains the N-terminal domain responsible for LPL inhibition. In the remainder of the paper, we will refer to the ANGPTL4 knockout first mice as Angptl $4^{\text {hyp }}$ mice, as explained below. To carefully characterize the Angptl ${ }^{\text {hyp }}$ mice, we performed qPCR for Angptl4 using cDNA obtained from adipose tissue and primary adipocytes of wild-type, Angptl $4^{\text {hyp }}$ and Angptl $^{-/ /}$mice. Primers were designed to amplify parts of the cDNA encoded by specific exons. 
A

B

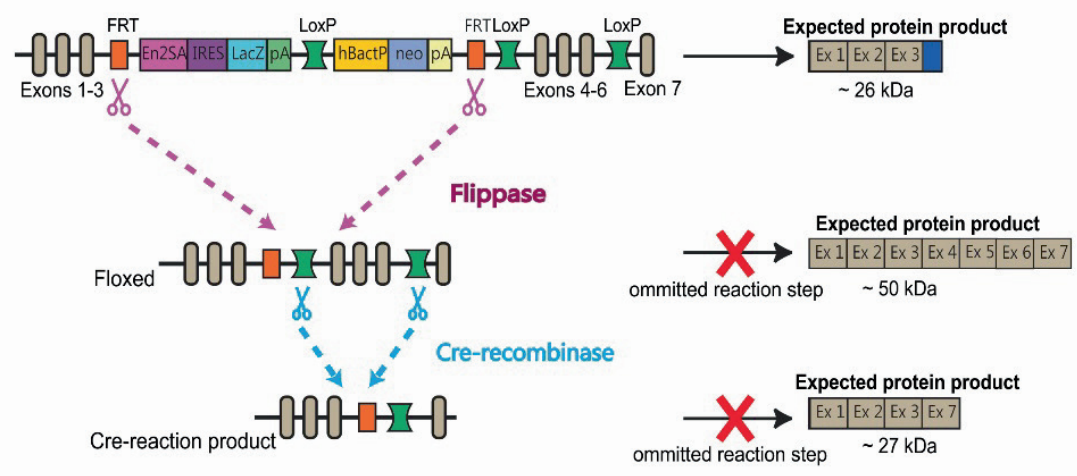

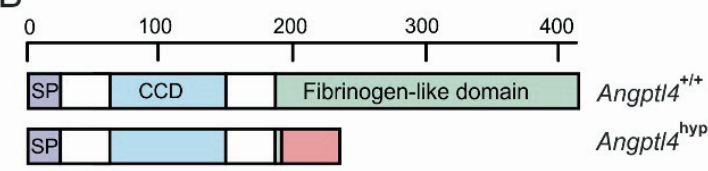
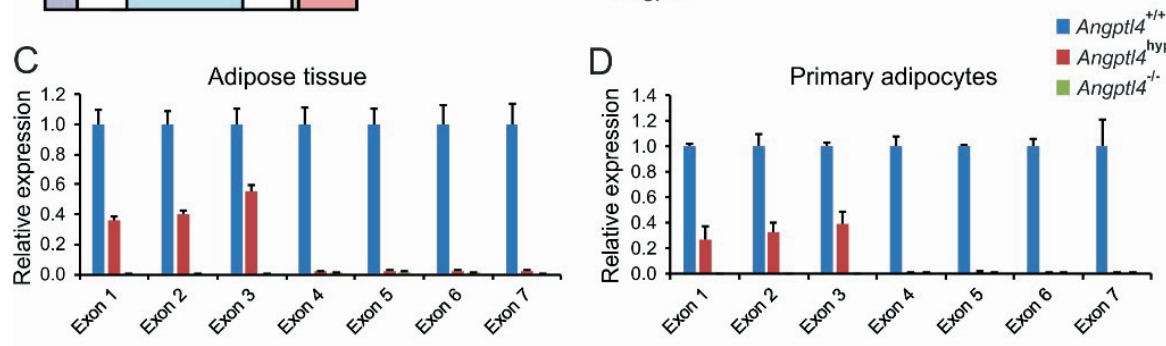

$E$
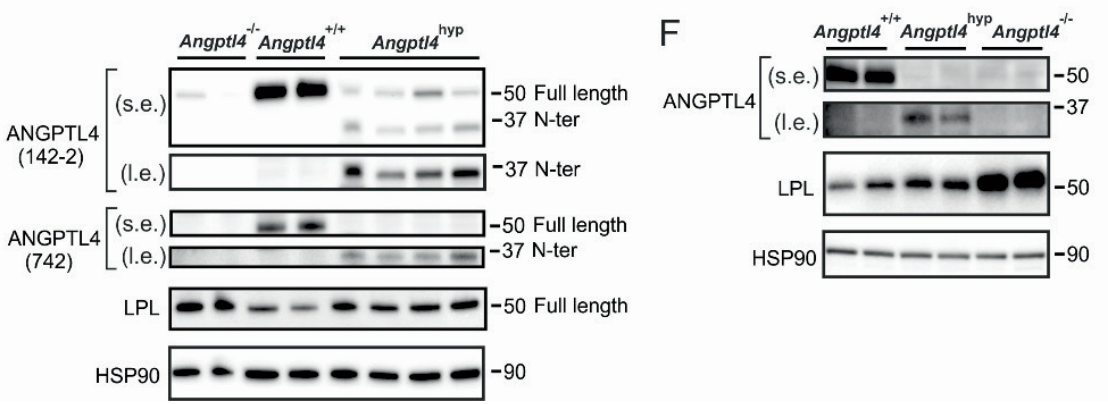

Figure 4. Hypomorphic ANGPTL4 mice show reduced ANGPTL4 expression and reduced regulation on LPL. (A) Schematic map of the Angptl4 locus by knockout first-allele strategy. (B) Full ANGPTL4 protein (upper scheme) and predicted sequence of truncated ANGPTL4 (lower scheme) based on sequence analysis of the knockout first-allele mice. mRNA expression of all 7 exons of Angptl4 in epididymal adipose tissue (C) and primary adipocytes (D) of Angptl4/- and Angptl $4^{\text {hyp }}$ mice relative to wild-type mice. Western blot showing full-length and N-terminal ANGPTL4, and LPL in adipose tissue (E) and primary adipocytes (F) of the Angptl4 ${ }^{-/}$, Angptl4 $4^{\text {hyp }}$ and wild-type mice. mRNA expression was normalized to $36 b 4$. $\mathrm{N}=7$ to 11 mice/group. Data are mean \pm SEM. 
Remarkably, whereas no Angptl4 expression using any of the primers could be detected in adipose tissue and adipocytes of Angptl4 ${ }^{-/}$mice, substantial Angptl4 expression (approx. 30-50\% of wild-type level) for exon 1-3 was found in the Angptl $4^{\text {hyp }}$ mice (Fig. 4C, D). By contrast, no amplification was observed in the Angptl ${ }^{\text {hyp }}$ mice using primers directed against parts of the cDNA encoded by exons 4-7 (Fig. 4C,D). A similar expression profile of the Angptl4 exons was observed in the mouse livers (Supplemental Fig. 1). These data indicate that the Angptl $4^{\text {hyp }}$ mice produce a truncated cDNA that may lead to the production of a truncated ANGPTL4 protein.

To verify this notion, we performed Western blot on adipose tissue of the three types of mice using a monoclonal antibody directed against mouse ANGPTL4. A strong ANGPTL4 band at $50 \mathrm{kD}$ was observed in the wild-type mice (Fig. 4E). At this position, a weak band was observed in the Angptl4 ${ }^{\text {hyp }}$ and Angptl $4^{-/-}$mice, which is likely nonspecific. Interestingly, a band around $32 \mathrm{kD}$ was observed in the Angptl ${ }^{\text {hyp }}$ mice but not in the Angptl4 ${ }^{-/ 2}$ mice (Fig. 4E). This band migrates at a molecular weight that is slightly higher than would be predicted for the ANGPTL4 fragment based on primary sequence. To verify that the designated bands indeed represent ANGPTL4, we used the same samples to perform Western blot using a polyclonal antibody directed against an epitope close to the N-terminus of ANGPTL4. Similar results were obtained (Fig. 4E). These analyses confirm that the Angptl4 $4^{\text {hyp }}$ mice produce a truncated ANGPTL4 protein containing the LPL inhibitory domain, at levels that are far below the levels in wild-type mice. Accordingly, the Angptl $4^{\text {hyp }}$ mice represent a hypomorph. In line with previous studies, we found that LPL protein levels are increased in adipose tissue of Angptl4 ${ }^{-1-}$ mice (Fig. 4E) [22,23]. LPL protein levels were also increased in Angptl4 ${ }^{\text {hyp }}$ mice (Fig. 4E). Similarly, primary adipocytes of Angptl $4^{\text {hyp }}$ mice expressed low levels of a truncated N-terminal ANGPTL4 protein (Fig. 4F). LPL protein levels in Angptl4hyp adipocytes were markedly lower than in Angptl $4^{-/-}$adipocytes, yet higher than in wild-type adipocytes (Fig. 4F).

\section{Angptl $^{\text {hyp }}$ and Angptl $^{-/-}$mice show similar regulation on plasma metabolites and LPL after 24-hour fast}

In line with the alternative name fasting-induced adipose factor, the effects of Angptl4 inactivation or overexpression on lipid metabolism are most prominent in the fasted state. Accordingly, we measured basic plasma parameters in the three types of mice in the fasted and fed state. Fasting plasma levels of triglycerides, cholesterol, and nonesterified fatty acids were remarkably similar between the Angptl $4^{\text {hyp }}$ and Angptl4 $4^{-/-}$mice, and were significantly lower compared to the wild-type mice (Fig. 5A). These data 
suggest that Angptl $4^{\text {hyp }}$ and Angptl $4^{-/}$mice are highly similar and that the low levels of N-terminal ANGPTL4 protein in the Angptl $4^{\text {hyp }}$ mice have no discernible impact on plasma lipid parameters.

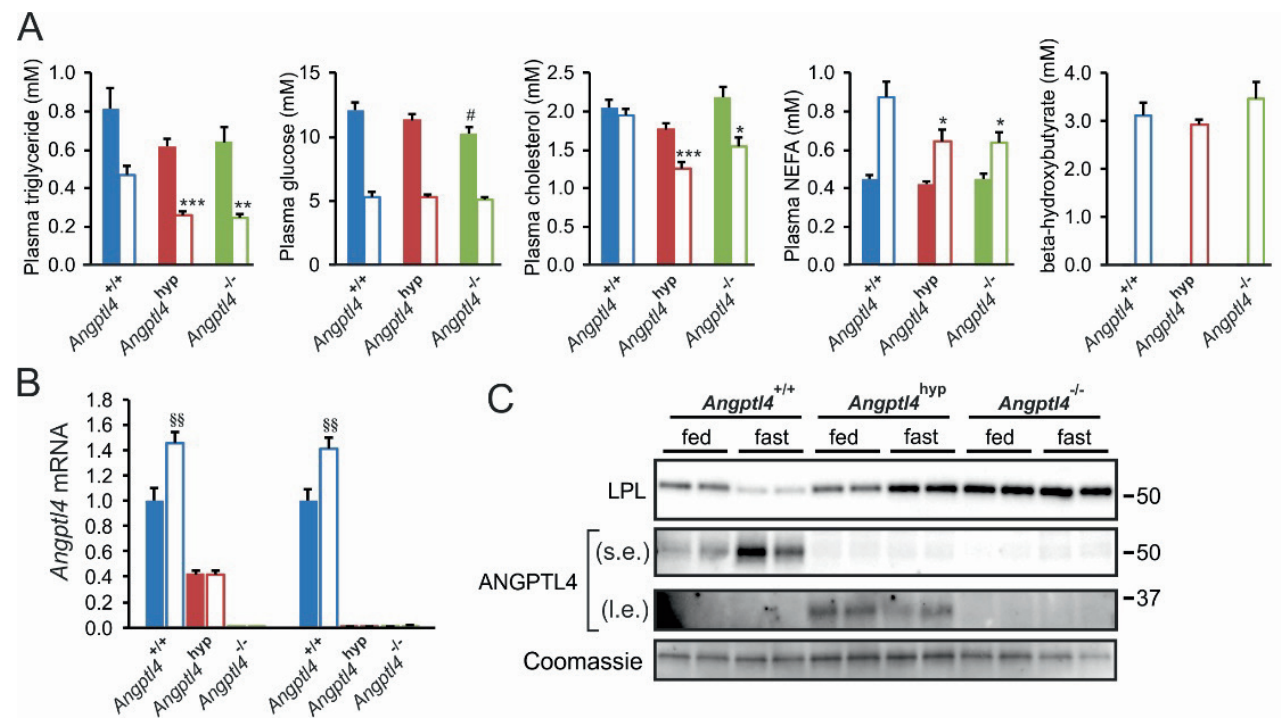

Figure 5. Similar regulation on plasma metabolites and LPL between Angptl $4^{\text {hyp }}$ and Angptl4 $^{-1-}$ mice after 24-hour fast. (A) Plasma levels of triglycerides, glucose, cholesterol, nonesterified fatty acids (NEFA) and $\beta$-hydroxybutyrate in fed (closed bars) and fasted (opened bars) mice. (B) Representative mRNA expression from N-terminal (left column) and C-terminal (right column) regions of Angptl4 in epididymal adipose tissues of fed (closed bars) and fasted (opened) mice. (C) Western blot of LPL and ANGPTL4 in epididymal adipose tissues from the 3 groups of mice in fed and fasted state. Coomassie staining represent loading control. mRNA expression was normalized to $36 b 4$. $\mathrm{N}=7$ to 11 mice/group. Data are mean $\pm \mathrm{SEM} ;{ }^{*} \mathrm{p}<0.05,{ }^{* *} \mathrm{p}<0.01$ and $* * * \mathrm{p}<0.001$ represent significance in fasted group relative to wild-type fasted mice; $\# \mathrm{p}<0.05$ represent significance in fed group relative to wild-type fed mice; and $\S \S p<0.01$ relative to wildtype fed mice.

Consistent with previous studies [28,32], Angptl4 mRNA in adipose tissue was significantly increased by fasting (Fig. 5B). Interestingly, fasting did not influence Angptl4 mRNA in the Angptl $4^{\text {hyp }}$ mice. In line with these data and fitting with previous studies [23,33], ANGPTL4 protein levels in wild-type adipose tissue were increased by fasting, whereas LPL protein levels were reduced by fasting (Fig. 5C). By contrast, levels of truncated ANGPTL4 protein were modestly reduced by fasting in the Angptl ${ }^{\text {hyp }}$ mice, concomitant with an increase in LPL protein. 
As previously shown [23], LPL protein was higher in Angptl4 ${ }^{-/}$mice compared with wild-type mice (Fig. 5C). Importantly, the decrease in LPL protein upon fasting was abrogated in Angptl4 ${ }^{-/}$mice, indicating that the increase in ANGPTL4 mediates the reduction in LPL protein upon fasting. Interestingly, in the fed state, LPL protein levels were lower in Angptl $4^{\text {hyp }}$ than Angptl $4^{-/-}$mice, whereas in the fasted, LPL protein levels were similar in Angptl $4^{\text {hyp }}$ and Angptl $4^{-/-}$mice (Fig. 5C). These data suggest that small amounts of N-terminal ANGPTL4 in adipose tissue can decrease LPL protein levels, presumably by promoting LPL degradation, specifically in the fed and not the fasted state.

\section{Angptl $^{\text {hyp }}$ mice show attenuated chylous ascites and acute inflammation but similar levels of lymphadenopathy as Angptl4 $^{-/-}$mice}

We have previously shown that Angptl $4^{-/-}$mice respond poorly to high saturated fat diet characterised by the formation of lipid-laden Touton giant cells in mesenteric lymph nodes, accumulation of chylous ascites in peritoneum and acute inflammation [25]. To determine the influence of low levels of N-terminal ANGPTL4 on the development of lymphadenopathy, chylous ascites and other pathologies, the three groups of mice were fed a standard high fat diet rich in saturated fat for 20 weeks. Whereas the Angptl $4^{-/ /}$mice showed a rapid and marked increase in serum amyloid A levels upon high fat feeding, serum amyloid A levels in the Angptl4 ${ }^{\text {hyp }}$ mice only went up after 20 weeks of high fat feeding (Fig. 6A). At this time point, hepatic expression of several acute phase proteins in Angptl ${ }^{\text {hyp }}$ mice was significantly lower than or approximating the levels in Angptl4 $4^{-1-}$ mice (Fig. 6B). Besides a delayed increase in serum amyloid A, the development of chylous ascites was much reduced in the Angptl $4^{\text {hyp }}$ mice compared to the Angptl $4^{-/-}$ mice. Whereas $90 \%$ of the Angptl $4^{-/}$mice show very pronounced ascites several weeks after starting the high fed diet, leading to premature death [25], at the end of the 20 weeks of high fat feeding only $12 \%$ of the Angptl $4^{\text {hyp }}$ mice showed any sign of ascites, which was also much milder (Fig. 6C). The mesenteric adipose tissue of the mice with ascites showed the characteristic mesenteric panniculitis (Fig. 6D). Of note, the frequency rate and severity of ascites in the Angptl $4^{\text {hyp }}$ mice remained unchanged when extending the high fat feeding to 36 weeks (data not shown). Despite the much milder ascites and slower progression of the inflammatory phenotype, after 20 weeks of high fat feeding nearly all mesenteric lymph nodes in the Angptl ${ }^{\text {hyp }}$ mice contained multiple lipid-laden Touton giant cells (Fig. 6E). 
A
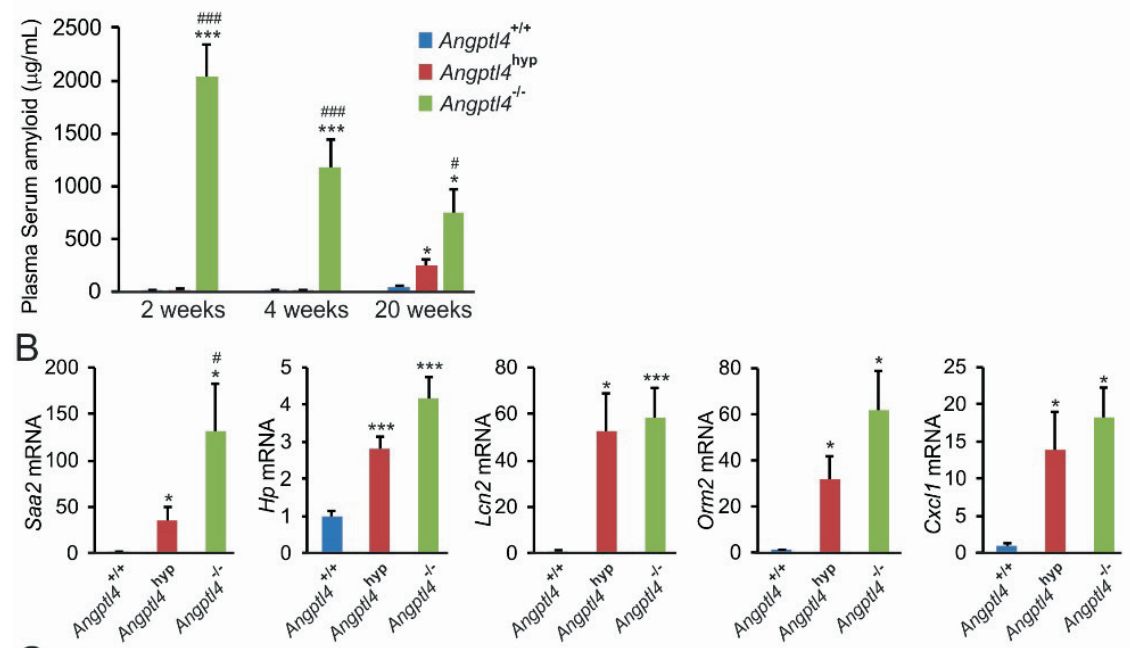

C
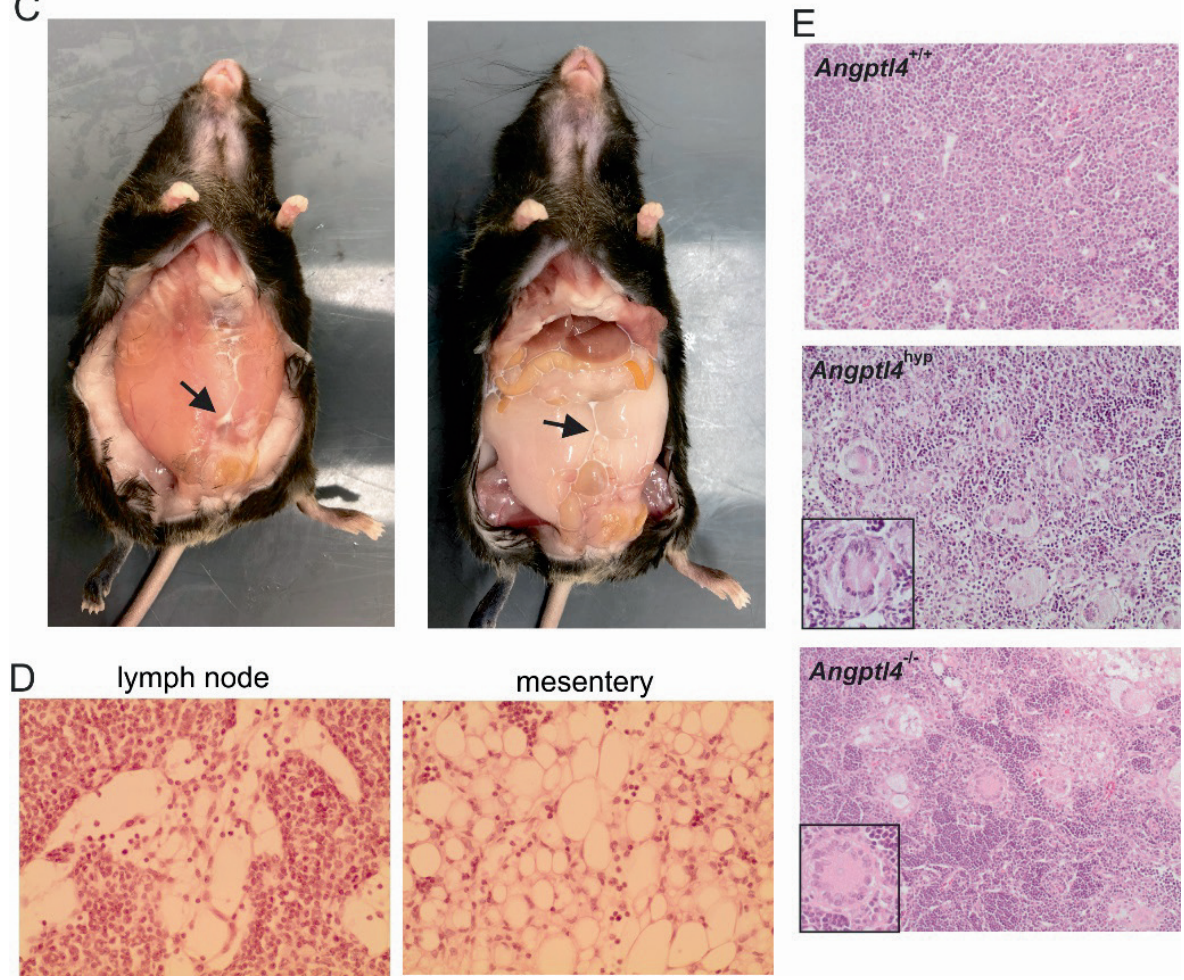

Figure 6. Angptl $4^{\text {hyp }}$ mice have less severe pathological phenotypes in response to 20-weeks of high fat diet compared to Angptl4 A- $^{--}$mice. (A) Plasma levels of serum amyloid A after 2, 4 and 20 weeks of high fat diet. (B) Relative hepatic expression of pro-inflammatory markers in three groups of mice. (C) Pictures showing levels of ascites formation in the peritoneum of 
Angptl ${ }^{\text {hyp }}$ mice. (D) Images depicting mesenteric panniculitis in Angptl4 ${ }^{\text {hyp }}$ mice. (E) Images showing Touton giant cells in the mesenteric lymph nodes of Angptl4 $4^{\text {hyp }}$ and Angptl4 ${ }^{-/}$mice but not wildtype mice. Images are 200x magnification; insert is $400 \mathrm{x}$ magnification. mRNA expression was normalized to $36 b 4 . \mathrm{N}=7$ to 11 mice/group. Data are mean $\pm \mathrm{SEM} ;{ }^{*} \mathrm{p}<0.05$, $* * \mathrm{p}<0.01$ and $* * * \mathrm{p}<0.001$ relative to wild-type.

\section{Angptl $^{\text {hyp }}$ mice show increased LPL activity and improved glucose tolerance compared to wild-type mice}

Consistent with the role of ANGPTL4 as LPL inhibitor, after 20 weeks of high fat feeding, adipose tissue LPL activity was significantly higher in Angptl $4^{\text {hyp }}$ mice than in wild-type mice (Fig. 7A). By contrast, adipose tissue LPL activity was not increased in Angptl $^{-/ /}$mice, which is likely related to the steatitis in these animals [24]. Interestingly, whereas plasma levels of triglycerides and cholesterol were significantly lower in both Angptl $4^{\text {hyp }}$ and Angptl4 ${ }^{-/-}$mice than in wild-type mice, plasma glycerol and non-esterified fatty acid levels were only lower in the Angptl $4^{\text {hyp }}$ mice, which again may be related to the steatitic phenotype of the Angptl4 ${ }^{-/}$adipose tissue (Fig. 7B). To study the impact of ANGPTL4 deficiency on glucose homeostasis, we measured plasma glucose and performed a glucose tolerance test. Plasma glucose levels were significantly lower in Angptl $4^{-/}$mice than in wild-type and Angptl ${ }^{\text {hyp }}$ mice (Fig. 7C), while glucose tolerance was dramatically or modestly improved in Angptl4 ${ }^{-/-}$and Angptl $4^{\text {hyp }}$ mice, respectively, compared with wild-type mice (Fig. 7D). Overall, the LPL activity and plasma metabolites levels in Angptl4 ${ }^{\text {hyp }}$ mice are consistent with the known role of ANGPTL4 in intra- and extracellular lipolysis. By contrast, LPL activity and plasma metabolite levels in Angptl $4^{-/-}$mice fed a high fat diet likely partly reflect the severe inflammatory phenotype in these animals. 

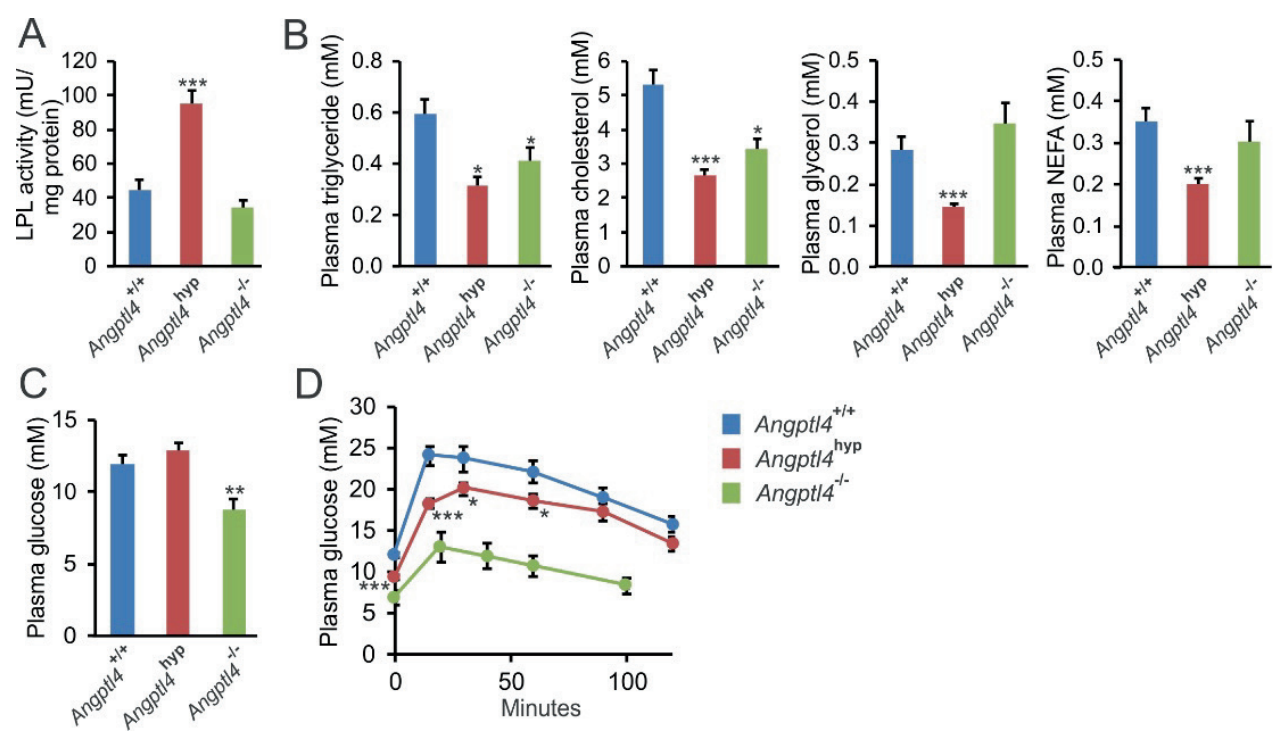

Figure 7. Increased LPL activity and improved glucose tolerance in Angptl ${ }^{\text {hyp }}$ mice after 20 week of high fat diet. (A) LPL activity in epididymal adipose tissue of Angptl4 ${ }^{\text {hyp }}$, Angptl4 ${ }^{-/}$and wild-type mice (B) Plasma concentrations of triglycerides, cholesterol, glycerol and non-esterified fatty acids (NEFA) in the 3 groups of mice. Plasma glucose levels (C) and glucose tolerance test (D) in the 3 groups of mice. $\mathrm{N}=7$ to 11 mice/group. Data are mean $\pm \mathrm{SEM} ;{ }^{*} \mathrm{p}<0.05,{ }^{* *} \mathrm{p}<0.01$ and $* * * \mathrm{p}<0.001$ relative to wild-type. No statistical analysis could be done on glucose tolerance in Angptl4 $^{-/}$mice due to the use of different time points. 


\section{Discussion}

In this paper we addressed two independent questions relevant to the inflammatory phenotype in ANGPTL4-deficient mice fed a high fat diet: 1) what is the influence of endogenous ANGPTL4 on lipid uptake in macrophages, and 2) what is the role of Nterminal ANGPTL4 in the development of the inflammatory phenotype. Previously, we showed that Angplt4 $4^{-/}$mice fed a diet rich in saturated fat develop an inflammatory and ultimately lethal phenotype characterized by the formation of Touton giant cells in mesenteric lymph nodes, chylous ascites, and fibrinopurulent peritonitis [24,25]. By comparing Angplt4 ${ }^{--}$mice with an Angptl4-hypomorphic model, here we show that a low level expression of a truncated N-terminal ANGPTL4 does not prevent Touton giant cell formation, yet drastically mitigates the acute phase response and the development of chylous ascites upon high fat feeding. Importantly, the development of fibrinopurulent peritonitis and the death of the animals is completely prevented by the expression of the truncated ANGPTL4. Although the severity of the clinical phenotype was attenuated, activation of the acute phase response and chylous ascites were still observed in a substantial number of Angptl4 ${ }^{\text {hyp }}$ mice. These data also indicate that the deleterious phenotype in Angplt4 ${ }^{-/}$mice fed a high fat diet is caused by the absence of the $\mathrm{N}$-terminal portion of ANGPTL4, which is involved in LPL inhibition, and is not related to the absence of the C-terminal fragment of ANGPTL4.

Our observation that partial deficiency of ANGPTL4 leads to undesirable clinical consequences in mice suggests that therapeutic approaches that only partially inactivate ANGPTL4 and/or are aimed at whole body inactivation of ANGPTL4 may still carry the risk of major side effects in humans. By contrast, tissue-specific inactivation, in particular adipose tissue-specific and possibly liver-specific inactivation of ANGPTL4, hold considerably more promise for improving dyslipidemia and reducing coronary artery disease risk without leading to deleterious side-effects [33].

In line with a predominant role of ANGPTL4 in lipid metabolism during fasting, plasma levels of triglycerides, cholesterol and non-esterified fatty acids were lower in Angptl $4^{-/-}$ mice compared with wild-type mice specifically in the fasted but not the fed state. Interestingly, in the fasted state, plasma triglyceride, cholesterol, and non-esterified fatty acid levels were similar in Angptl ${ }^{\text {hyp }}$ and Angptl $4^{-/-}$mice, which was accompanied by similar levels of LPL protein in the adipose tissue. Accordingly, the expression of low levels of N-terminal ANGPTL4 does not influence LPL protein levels in the fasted state. By contrast, in the fed state, LPL protein levels in adipose tissue were lower in Angptl $4^{\text {hyp }}$ mice than in Angptl4 ${ }^{-/-}$mice, suggesting that a low level of N-terminal ANGPTL4 
decreases LPL protein, likely by promoting LPL degradation. The reason why Nterminal ANGPTL4 in Angptl $4^{\text {hyp }}$ mice has a more pronounced effect on LPL in the fed state than in the fasted state could be related to the decrease in N-terminal ANGPTL4 during fasting. This decrease in N-terminal ANGPTL4 in Angptl4 $4^{\text {hyp }}$ mice by fasting likely accounts for the parallel increase in LPL protein. Why N-terminal ANGPTL4 is suppressed by fasting in Angptl $4^{\text {hyp }}$ mice is unclear but does not seem to be mediated at the transcriptional level.

In addition to regulating plasma lipid levels, recent reports also suggest a role of ANGPTL4 in glucose homeostasis [34-36]. Using different models, ANGPTL4 deficiency in mice was found to lead to improved glucose tolerance. Consistent with the study by Gusarova et al [37], we found that Angptl $4^{-/-}$mice chronically fed a high fat diet have drastically lower basal plasma glucose levels and improved glucose tolerance. However, these mice have numerous clinical abnormalities, which prohibits any conclusion on the direct effect of ANGPTL4 deficiency on glucose homeostasis. Angptl ${ }^{\text {hyp }}$ mice fed a high fat diet showed a much milder clinical phenotype, concomitant with a more modest yet statistically significant improvement in glucose tolerance, yet acute phase protein levels were still elevated compared with wild-type mice. This observation again makes it difficult to derive a solid conclusion on the direct effect of ANGPTL4 deficiency on glucose homeostasis. We previously found that Angptl $^{-/ /}$mice fed a diet high in unsaturated fat, which does not exhibit any clinical complications, were more glucose tolerant than wild-type mice fed the same diet [35]. In addition, it was shown that adipocyte-specific ANGPTL4-deficient mice have markedly improved glucose and insulin tolerance after one month and to a lesser extent five months of high fat feeding, which was suggested to be due to LPL-mediated redistribution of ectopic fat stores to adipose tissue [34]. While growing evidence thus connects ANGPTL4 with glucose homeostasis, further studies are necessary to determine how ANGPTL4 may influence glucose homeostasis.

An interesting question concerns the significance of the lipid-laden Touton giant cells for the onset of the debilitating side-effects after high fat feeding. A causal role had been previously questioned by the observation that Angptl4 ${ }^{-/ 2}$ mice fed a high trans-fat diet develop Touton cells but do not show elevated systemic inflammation or ascites and survive the intervention [25]. Here we find that Angptl $4^{\text {hyp }}$ mice fed a high fat diet, despite showing a much milder inflammation and ascites than Angptl $4^{-/}$mice, carry similar numbers of Touton giant cells in their mesenteric lymph nodes. These data reinforce the notion that the formation of lipid-laden Touton giant cells is uncoupled from activation of an acute-phase response and chylous ascites [25]. 
Our study confirms previous data indicating that Angptl4 expression in macrophages is highly induced by lipids $[24,38,39]$. This regulation is likely mediated by PPAR $\delta$ and/or PPAR $\gamma$, as shown by the marked induction of Angptl4 mRNA by PPAR $\delta$ and PPAR $\gamma$ agonists in bone marrow-derived macrophages. Importantly, the present study shows that not only external ANGPTL4 but also endogenously produced ANGPTL4 inhibits lipid uptake in macrophages. The elevated lipid uptake in Angptl $4^{-/-}$macrophages increases the expression of various lipid-sensitive genes involved in inflammation and ER stress, such as Hilpda, Ddit3, Ptgs2 and Cxcl2. The absence of ANGPTL4 in bone marrowderived macrophages did not influence respiration and fuel utilization, indicating that the influence of ANGPTL4 deficiency on inflammation is likely not due to changes in intracellular metabolism. Together, our findings solidify the important role of ANGPTL4 in protecting macrophages from the deleterious effects of excessive lipid uptake.

It is well established that ANGPTL4 inactivates LPL by promoting LPL unfolding $[19,40]$. Recently, we demonstrated a local role of action for ANGPTL4 in promoting the intracellular cleavage and subsequent degradation of LPL in adipocytes [22]. In stark contrast, ANGPTL4 deficiency in macrophages does not lead to the accumulation of full length, rapidly-releasable LPL, while the cleaved, N-terminal LPL portion is generally not detectable in our hands. These data suggest that the inhibitory effect of ANGPTL4 on LPL activity in macrophages does not involve enhanced LPL degradation. The cellular factors explaining the difference in ANGPTL4 action between adipocytes and macrophages remain to be identified. Overall, these findings suggest that the location and cellular mechanism of LPL inactivation by ANGPTL4 may be different in different tissues.

In conclusion, we find that ANGPTL4 deficiency enhances lipid uptake into macrophages. Furthermore, in comparison to Angptl $4^{-/-}$mice, mice expressing low levels of N-terminal ANGPTL4 show a reduced acute phase response and milder chylous ascites following high fat feeding. These findings have significant clinical implications since any therapeutic strategy would likely reduce but not completely inactivate ANGPTL4. 


\section{Acknowledgements}

We thank Madelene Ericsson and Rakel Nyrén (Department of Medical Biosciences/Physiological Chemistry, Umeå University, Sweden) for their technical assistance with the LPL activity measurements, and Imke Vohs (Wageningen University) for her assistance with the H\&E staining.

Funding: This work was supported by the Graduate School Voeding, Levensmiddelentechnologie, Agro-Biotechnologie en Gezondheid (VLAG) (Wageningen University) and CVON ENERGISE grant CVON2014-02.

Disclosure: The authors declare that there is no conflict of interest associated with this manuscript. 


\section{References}

[1] Singh, A., Singh, R., 2016. Triglyceride and cardiovascular risk: A critical appraisal. Indian Journal of Endocrinology and Metabolism 20(4): 418.

[2] Reiner, Ž., 2017. Hypertriglyceridaemia and risk of coronary artery disease. Nature Reviews Cardiology.

[3] Sandesara, P.B., Virani, S.S., Fazio, S., Shapiro, M.D., 2018. The Forgotten Lipids: Triglycerides, Remnant Cholesterol, and Atherosclerotic Cardiovascular Disease Risk. Endocrine Reviews.

[4] Toth, P.P., 2016. Triglyceride-rich lipoproteins as a causal factor for cardiovascular disease. Vascular Health and Risk Management 12: 171-83.

[5] Kersten, S., 2014. Physiological regulation of lipoprotein lipase. Biochimica et Biophysica Acta 1841(7): 919-33.

[6] Beigneux, A.P., Miyashita, K., Ploug, M., Blom, D.J., Ai, M., Linton, M.F., et al., 2017. Autoantibodies against GPIHBP1 as a Cause of Hypertriglyceridemia. New England Journal of Medicine.

[7] Li, Y., He, P.P., Zhang, D.W., Zheng, X.L., Cayabyab, F.S., Yin, W.D., et al., 2014. Lipoprotein lipase: From gene to atherosclerosis. Atherosclerosis 237(2): 597-608.

[8] Davies, B.S.J., Beigneux, A.P., Ii, R.H.B., Tu, Y., Gin, P., Weinstein, M.M., et al., 2011. GPIHBP1 Is Responsible for the Entry of Lipoprotein Lipase into Capillaries. Cell 12(1): 42-52.

[9] Merkel M, Eckel, R. and G.I., 2002. Lipoprotein lipase: genetics, lipid uptake, and regulation. J. Lipid Res.: 1997-2006.

[10] Dijk, W., Kersten, S., 2014. Regulation of lipoprotein lipase by Angpt14. Trends in Endocrinology and Metabolism 25(3): 146-55.

[11] Mattijssen, F., Kersten, S., 2012. Regulation of triglyceride metabolism by Angiopoietin-like proteins. Biochimica et Biophysica Acta 1821(5): 782-9.

[12] Shan, L., Yu, X.C., Liu, Z., Hu, Y., Sturgis, L.T., Miranda, M.L., et al., 2009. The angiopoietin-like proteins ANGPTL3 and ANGPTL4 Inhibit lipoprotein lipase activity through distinct mechanisms. Journal of Biological Chemistry 284(3): 141924.

[13] Kersten, S., 2017. Angiopoietin-like 3 in lipoprotein metabolism. Nature Reviews Endocrinology.

[14] Abid, K., Trimeche, T., Mili, D., Msolli, M.A., Trabelsi, I., Nouira, S., et al., 2016. ANGPTL4 variants E40K and T266M are associated with lower fasting triglyceride levels and predicts cardiovascular disease risk in Type 2 diabetic Tunisian population. Lipids in Health and Disease 15(1): 63.

[15] Dewey, F.E., Gusarova, V., O’Dushlaine, C., Gottesman, O., Trejos, J., Hunt, C., et al., 2016. Inactivating Variants in ANGPTL4 and Risk of Coronary Artery Disease. New England Journal of Medicine 374(12): 1123-33.

[16] Köster, A., Chao, Y.B., Mosior, M., Ford, A., Gonzalez-DeWhitt, P.A., Hale, J.E., et al., 2005. Transgenic angiopoietin-like (Angptl)4 overexpression and targeted 
disruption of Angpt14 and Angptl3: Regulation of triglyceride metabolism. Endocrinology 146(11): 4943-50.

[17] Mandard, S., Zandbergen, F., Van Straten, E., Wahli, W., Kuipers, F., Müller, M., et al., 2006. The fasting-induced adipose factor/angiopoietin-like protein 4 is physically associated with lipoproteins and governs plasma lipid levels and adiposity. Journal of Biological Chemistry 281(2): 934-44.

[18] Dijk, W., Kersten, S., 2016. Regulation of lipid metabolism by angiopoietin-like proteins. Current Opinion in Lipidology 27: 249-256.

[19] Mysling, S., Kristensen, K.K., Larsson, M., Kovrov, O., Bensadouen, A., Jørgensen, T.J.D., et al., 2016. The angiopoietin-like protein angptl4 catalyzes unfolding of the hydrolase domain in lipoprotein lipase and the endothelial membrane protein gpihbp1 counteracts this unfolding. ELife 5(DECEMBER2016): 18.

[20] Sukonina, V., Lookene, A., Olivecrona, T., Olivecrona, G., 2006. Angiopoietin-like protein 4 converts lipoprotein lipase to inactive monomers and modulates lipase activity in adipose tissue. Proceedings of the National Academy of Sciences.

[21] Lichtenstein, L., Berbée, J.F.P., Van Dijk, S.J., Van Dijk, K.W., Bensadoun, A., Kema, I.P., et al., 2007. Angptl4 upregulates cholesterol synthesis in liver via inhibition of LPL- and HL-dependent hepatic cholesterol uptake. Arteriosclerosis, Thrombosis, and Vascular Biology 27(11): 2420-7.

[22] Dijk, W., Ruppert, P.M.M., Oost, L.J., Kersten, S., 2018. Angiopoietin-like 4 promotes the intracellular cleavage of lipoprotein lipase by PCSK3/furin in adipocytes. Journal of Biological Chemistry 293(36): 14134-45.

[23] Dijk, W., Beigneux, A.P., Larsson, M., Bensadoun, A., Young, S.G., Kersten, S., 2016. Angiopoietin-like 4 (ANGPTL4) promotes intracellular degradation of lipoprotein lipase in adipocytes. Journal of Lipid Research 7(11): 956-63.

[24] Lichtenstein, L., Mattijssen, F., de Wit, N.J., Georgiadi, A., Hooiveld, G.J., van der Meer, R., et al., 2010. Angpt14 Protects against Severe Proinflammatory Effects of Saturated Fat by Inhibiting Fatty Acid Uptake into Mesenteric Lymph Node Macrophages. Cell Metabolism 12(6): 580-92.

[25] Oteng, A.-B., Bhattacharya, A., Brodesser, S., Qi, L., Tan, N.S., 2017. Feeding Angptl4- / - mice trans fat promotes foam cell formation in mesenteric lymph nodes without leading to ascites. J. Lipid Res. 58: 1-36.

[26] Dijk, W., Heine, M., Vergnes, L., Boon, M.R., Schaart, G., Hesselink, M.K.C., et al., 2015. ANGPTL4 mediates shuttling of lipid fuel to brown adipose tissue during sustained cold exposure. ELife 4(OCTOBER2015): 1-23.

[27] Skarnes, W.C., Rosen, B., West, A.P., Koutsourakis, M., Bushell, W., Iyer, V., et al., 2011. A conditional knockout resource for the genome-wide study of mouse gene function. Nature 474.

[28] Kroupa, O., Vorrsjö, E., Stienstra, R., Mattijssen, F., Nilsson, S.K., Kersten, S., et al., 2012. Linking nutritional regulation of Angptl4, Gpihbp1, and Lmf1 to lipoprotein lipase activity in rodent adipose tissue: $1-15$.

[29] Markwell, M.A.K., Haas, S.M., Bieber, L.L., Tolbert, N.E., 1978. A modification of the Lowry procedure to simplify protein determination in membrane and lipoprotein 
samples. Analytical Biochemistry 87(1): 206-10.

[30] Mandard, S., Zandbergen, F., Nguan, S.T., Escher, P., Patsouris, D., Koenig, W., et al., 2004. The direct peroxisome proliferator-activated receptor target fastinginduced adipose factor (FIAF/PGAR/ANGPTL4) is present in blood plasma as a truncated protein that is increased by fenofibrate treatment. Journal of Biological Chemistry 279(33): 34411-20.

[31] Robblee, M.M., Kim, C.C., Abate, J.P., Valdearcos, M., Sandlund, K.L.M., Shenoy, M.K., et al., 2016. Saturated Fatty Acids Engage an IRE1 $\alpha$-Dependent Pathway to Activate the NLRP3 Inflammasome in Myeloid Cells. Cell Reports: 1-13.

[32] Kersten, S., Mandard, S., Tan, N.S., Escher, P., Metzger, D., Chambon, P., et al., 2000. Characterization of the fasting-induced adipose factor FIAF, a novel peroxisome proliferator-activated receptor target gene. Journal of Biological Chemistry 275(37): 28488-93.

[33] Doolittle, M.H., Ben-Zeev, O., Elovson, J., Martin, K., Kirchgessner., 1990. The response of lipoprotein lipase to feeding and fasting. Journal of Biological Chemistry 265(8): 4570-7.

[34] Aryal, B., Singh, A.K., Zhang, X., Varela, L., Rotllan, N., Goedeke, L., et al., 2018. Absence of ANGPTL4 in adipose tissue improves glucose tolerance and attenuates atherogenesis. JCI Inisght 3(6): 1-19.

[35] Janssen, A.W.F., Katiraei, S., Bartosinska, B., Eberhard, D., Dijk, K.W. Van., 2018. Loss of angiopoietin-like 4 ( ANGPTL4) in mice with diet-induced obesity uncouples visceral obesity from glucose intolerance partly via the gut microbiota. Diabetologia 61(6): 1447-1458.

[36] Davies, B.S.J., 2018. Can targeting ANGPTL proteins improve glucose tolerance? Diabetologia 61(6): 1277-1281.

[37] Gusarova, V. et al., 2018. Genetic inactivation of ANGPTL4 improves glucose homeostasis and is associated with reduced risk of diabetes. Nature Communications 9(2252): 1-11.

[38] Georgiadi, A., Wang, Y., Stienstra, R., Tjeerdema, N., Janssen, A., Stalenhoef, A., et al., 2013. Overexpression of angiopoietin-like protein 4 protects against atherosclerosis development. Arteriosclerosis, Thrombosis, and Vascular Biology 33(7): 1529-37.

[39] Aryal, B., Rotllan, N., Araldi, E., Ramírez, C.M., He, S., Chousterman, B.G., et al., 2016. ANGPTL4 deficiency in haematopoietic cells promotes monocyte expansion and atherosclerosis progression. Nature Communications 7.

[40] Sukonina, V., Lookene, A., Olivecrona, T., Olivecrona, G., 2006. Angiopoietin-like protein 4 converts lipoprotein lipase to inactive monomers and modulates lipase activity in adipose tissue. Pnas 103(46): 17450-5. 


\section{Supplemental Material}

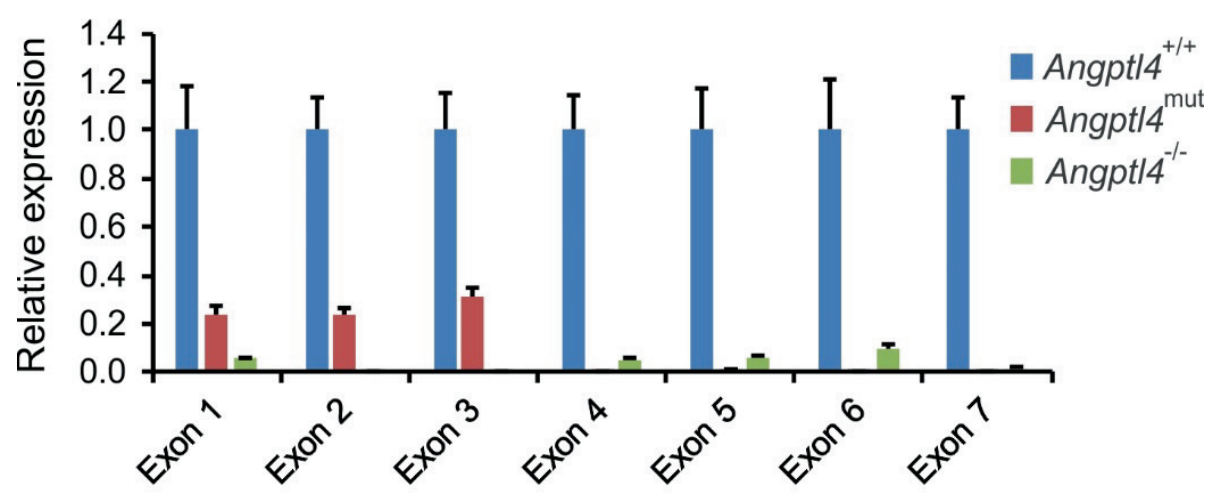

Supplemental Figure 1: Relative mRNA expression of all 7 exons of Angptl4 in the liver Angptl4 ${ }^{-/}$, Angptl4 ${ }^{\text {mut }}$ and wild-type mice. mRNA expression was normalized to $36 \mathrm{~b} 4$. N=7-11 mice/group. Data are mean \pm SEM. 


\section{Supplemental Table 1: Primer sequences of genes}

\begin{tabular}{|c|c|c|}
\hline$\underline{\text { Genes }}$ & Forward primer & Reverse primer \\
\hline m36B4 & ATGGGTACAAGCGCGTCCTG & GCCTTGACCTTTTCAGTAAG \\
\hline mAngptl4 & GTTTGCAGACTCAGCTCAAGG & CCAAGAGGTCTATCTGGCTCTG \\
\hline mHilpda & GCACGACCTGGTGTGACTGT & CCAGCACATAGAGGTTCAGCAT \\
\hline mDdit3 & CTGGAAGCCTGGTATGAGGAT & CAGGGTCAAGAGTAGTGAAGGT \\
\hline mXbp1s & AAACAGAGTAGCAGCGCAGACTGC & GGATCTCTAAAACTAGAGGCTTGGTG \\
\hline $\mathrm{mCxcl} 2 \mathrm{C}$ & CCAACCACCAGGCTACAGG & GCGTCACACTCAAGCTCTG \\
\hline mErn1 & ACACCGACCACCGTATCTCA & CTCAGGATAATGGTAGCCATGTC \\
\hline $\mathrm{mPlin} 2 \mathrm{C}$ & CTTGTGTCCTCCGCTTATGTC & GCAGAGGTCACGGTCTTCAC \\
\hline mCxcl1 & CTGGGATTCACCTCAAGAACATC & CAGGGTCAAGGCAAGCCTC \\
\hline $\mathrm{mCcr} 1$ & CTCATGCAGCATAGGAGGCTT & ACATGGCATCACCAAAAATCCA \\
\hline mPtgs 2 & TTCAACACACTCTATCACTGGC & AGAAGCGTTTGCGGTACTCAT \\
\hline $\mathrm{mCd} 14$ & CACACTCACTCAАCТTTТССТ & GCTGAGATCAGTCCTCTCTCG \\
\hline mGdf15 & CTGGCAATGCCTGAACAACG & GGTCGGGACTTGGTTCTGAG \\
\hline mAngtpl4 l & Exon1 CTTTGCATCCTGGGACGAGATG & GACAAGCGTTACCACAGGCAG \\
\hline mAngtpl4 & Exon2 GCTCAAGGCTCAAAACAGCAA & TGATAGGTATCTCTGCTGCTGG \\
\hline mAngtp14 & Exon3 CCCACGCACCTAGACAATGG & TGGGAGTCAAGCCAATGAGC \\
\hline mAngtpl4 & Exon4 GGGACTGCCAGGAACTCTTC & AATGGTGGAGACCCCAGAGG \\
\hline mAngtp14 & Exon5 GAGGCTGGACAGTGATTCAGAGA & GGGATCTCCGAAGCCATCCTT \\
\hline mAngtp14 & Exon6 ACGGCCAATGAGCTGGG & GGGCAGGGAAAGGCCA \\
\hline mAngtp14 & Exon7 CGCTACTATCCTCTGCAGGC & CCCTCGAGCCCATGTTTTCT \\
\hline mSaa2 & GCGAGCCTACACTGACATGA & TTTTCTCAGCAGCCCAGACT \\
\hline $\mathrm{mHp}$ & GCTATGTGGAGCACTTGGTTC & CACCCATTGCTTCTCGTCGTT \\
\hline $\operatorname{mLcn} 2$ & TGGAAGAACCAAGGAGCTGT & GGTGGGGACAGAGAAGATGA \\
\hline Orm2 & CAACATCACCATAGGCGACCC & ATTTCCTGCCGGTAATCAGGG \\
\hline
\end{tabular}


Chapter 6 


\section{Muscle-specific inflammation}

induced by MCP-1 overexpression does not affect whole-body insulin sensitivity in mice

Inkie J. A. Evers-van Gogh*, Antwi-Boasiako Oteng*, Sheril Alex, Nicole Hamers, Milene Catoire, Rinke Stienstra, Eric Kalkhoven \& Sander Kersten

*Authors contributed equally

Diabetologia. 2016; 59: 624-633. 


\section{Abstract}

Aims/hypothesis Obesity is associated with a state of chronic low-grade inflammation that is believed to contribute to the development of skeletal muscle insulin resistance. However, the extent to which local and systemic elevation of cytokines, such as monocyte chemoattractant protein 1 (MCP-1), interferes with the action of insulin and promotes insulin resistance and glucose intolerance in muscle remains unclear. Here, we aim to investigate the effect of muscle-specific overexpression of MCP-1 on insulin sensitivity and glucose tolerance in lean and obese mice.

Methods We used Mck-Mcp-1 transgenic (Tg) mice characterised by muscle-specific overexpression of $\mathrm{Mcp}-1$ (also known as $C c l 2$ ) and elevated plasma MCP-1 levels. Mice were fed either chow or high-fat diet for 10 weeks. Numerous metabolic variables were measured, including glucose and insulin tolerance tests, muscle insulin signalling and plasma NEFA, triacylglycerol, cholesterol, glucose and insulin.

Results Despite clearly promoting skeletal muscle inflammation, muscle-specific overexpression of $M c p-1$ did not influence glucose tolerance or insulin sensitivity in either lean chow-fed or diet-induced obese mice. In addition, plasma NEFA, triacylglycerol, cholesterol, glucose and insulin were not affected by MCP-1 overexpression. Finally, in vivo insulin-induced Akt phosphorylation in skeletal muscle did not differ between Mcp-1-Tg and wild-type mice.

Conclusions/interpretation We show that increased MCP- 1 production in skeletal muscle and concomitant elevated MCP-1 levels in plasma promote inflammation in skeletal muscle but do not influence insulin signalling and have no effect on insulin resistance and glucose tolerance in lean and obese mice. Overall, our data argue against MCP-1 promoting insulin resistance in skeletal muscle and raise questions about the impact of inflammation on insulin sensitivity in muscle. 


\section{Introduction}

Over recent decades, the global prevalence of obesity has been increasing steadily, concurrent with major changes in diet and lifestyle. Obesity is often accompanied by a number of metabolic perturbations, including insulin resistance, hyperinsulinaemia, dyslipidaemia and hypertension, which together substantially increase the risk for type 2 diabetes and cardiovascular disease [1-4]. Development of insulin resistance is generally considered as an early event that drives many of the metabolic disturbances associated with obesity. Since insulin resistance was first recognised several decades ago, a number of ideas have been put forward to try and explain the link between excess adiposity and reduced tissue sensitivity to insulin. Currently, major emphasis is placed on the detrimental effect of fat stored outside the adipose compartment. Additionally and alternatively, inflammation is increasingly believed to play a pivotal role in the development of insulin resistance and type 2 diabetes (reviewed in [5]). According to this so-called inflammatory hypothesis, obesity is characterised by expansion of the adipose depot, giving rise to adipocyte hypertrophy, hypoxia and endoplasmic reticulum (ER) stress, which in turn causes enhanced production of cytokines and chemokines [68]. Cytokines such as TNF $\alpha$, IL-1 $\beta$, IL-6, IL-8 and monocyte chemoattractant protein 1 (MCP-1) promote the attraction of different immune cells in obese adipose tissue, including proinflammatory macrophages and $\mathrm{B}$ cells, whereas regulatory $\mathrm{T}$ cells, natural killer $\mathrm{T}$ cells and eosinophils become less abundant [9]. These immune cells are producers of a host of different cytokines/chemokines and together with the hypertrophic adipocytes contribute to a state of chronic low-grade inflammation. In vitro studies abound indicating that proinflammatory cytokines are able to inhibit insulin signalling and thus promote the development of insulin resistance in fat cells and muscle cells. For example, Eckel and colleagues found that the chemokine MCP-1 impaired insulin signalling and reduced insulin-stimulated glucose uptake in muscle cells, suggesting that MCP-1 may represent a molecular link in the negative crosstalk between adipose tissue and skeletal muscle [10]. What is less clear is whether the obesity-induced adipose tissue inflammation leads to the increments in cytokine levels in blood plasma necessary to interfere with the action of insulin and to cause insulin resistance in muscle in vivo. This is particularly relevant since it has been calculated that approximately $80 \%$ of insulin-induced glucose disposal is accounted for by skeletal muscle glucose uptake. Studies performed using mice characterised by fat-specific overexpression of $\mathrm{Mcp}-1$ (also known as $C c l 2$ ) or expression of a dominant-negative mutant of $M c p-1$ suggest that fat-derived MCP-1 reduces glucose tolerance and insulin sensitivity and blunts 
insulin-mediated signalling $[11,12]$. However, the full scope of the role of adipose tissue inflammation and the impact of specific cytokines such as MCP-1 on whole-body glucose tolerance and insulin's action in skeletal muscle remains ambiguous.

Importantly, recent studies indicate that elevated MCP-1 expression not only occurs during obesity but is also a feature of physical exercise. Specifically, levels of MCP-1 mRNA in skeletal muscle and levels of MCP-1 protein in plasma were found to be increased upon acute exercise, leading to the classification of MCP-1 as a myokine or exercise factor [13-17]. In skeletal muscle, MCP-1 is known to promote macrophage infiltration after (severe) tissue damage [18,19]. In contrast, chronic exercise results in a decrease in MCP-1 plasma levels [20,21]. The features of MCP-1 are reminiscent of IL-6. Production of IL-6 in adipose tissue has been shown to be increased during obesity and contributes to obesity-associated low-grade inflammation (reviewed in [22]). At the same time, production of IL-6 in skeletal muscle and plasma IL-6 levels are increased in response to acute exercise and have been suggested to potentially confer a positive effect on insulin signalling and insulin sensitivity in skeletal muscle [23], although recent data argue against that notion [24]. How inflammatory mediators may have a beneficial role in the context of physical exercise yet promote insulin resistance during obesity represents somewhat of a conundrum.

The aim of the present study was to assess the extent to which elevation of MCP-1 production in muscle and elevated MCP-1 levels in plasma may be able to interfere with insulin signalling in skeletal muscle and promote insulin resistance. To this end, we took advantage of $M c k-M c p-1$ transgenic (Tg) mice, which specifically overexpress $M c p-1$ in skeletal muscle. Our studies indicate that elevation of MCP-1 production in skeletal muscle and elevated MCP-1 levels in plasma promote inflammation in skeletal muscle but do not influence insulin signalling in skeletal muscle and have no effect on insulin resistance and glucose tolerance in lean and obese mice. 


\section{Materials and Methods}

\section{Animals}

Animal studies were carried out using wild-type (WT) and $M c k-M c p-1$ Tg mice, all on a C57Bl/6J background [25]. The mice were kindly donated by D. Patsouris (Université Claude Bernard Lyon 1, France). Only male mice were used. Mice were kept in temperature-controlled rooms $\left(21 \pm 1^{\circ} \mathrm{C}\right)$ on a $12 \mathrm{~h}$ light-dark cycle. For the studies using chow-fed mice, $16 \mathrm{WT}$ and 19 Mcp-1-Tg mice were fed regular chow (RMH-B Arie Blok, Woerden, the Netherlands). At 14-17 weeks of age, eight mice per group were subjected to an intraperitoneal glucose tolerance test and a week later to an intraperitoneal insulin tolerance test. Mice were euthanised at 17-20 weeks of age after a $6 \mathrm{~h}$ fast at zeitgeber time (ZT)7-ZT9. Before being euthanised, eight mice of each genotype received an intraperitoneal injection of insulin ( $2 \mathrm{U} / \mathrm{kg}$ body weight in $100 \mu \mathrm{l}$ saline $[154 \mathrm{mmol} / \mathrm{l} \mathrm{NaCl}], \mathrm{n}=4$ per genotype $)$ or saline $(100 \mu \mathrm{l}, \mathrm{n}=4$ per genotype followed by euthanasia exactly $5 \mathrm{~min}$ later by cervical dislocation. For the remaining mice, blood was collected by orbital puncture under isoflurane anaesthesia and mice were euthanised by cervical dislocation. For the high-fat feeding study, 12 WT and 12 Mcp-1-Tg mice were fed regular chow (RMH-B Arie Blok) after weaning. At 12-16 weeks of age, mice were switched to a high-fat diet (HFD) containing 60 energy $\%$ fat (D12492; Research Diets Inc., New Brunswick, NJ, USA). Food intake and body weights were registered weekly. After 8 weeks of high-fat feeding, mice were subjected to an intraperitoneal glucose tolerance test and a week later to an intraperitoneal insulin tolerance test. Mice were euthanised after 10 weeks of high-fat feeding after a $6 \mathrm{~h}$ fast at ZT7-ZT9. Before being euthanised, four mice of each genotype received an intraperitoneal injection of insulin $(2 \mathrm{U} / \mathrm{kg}$ body weight in $100 \mu \mathrm{l}$ saline, $\mathrm{n}=2$ per genotype) or saline ( $100 \mu \mathrm{l}, \mathrm{n}=2$ per genotype) followed by euthanasia exactly $5 \mathrm{~min}$ later by cervical dislocation. For the remainder of the mice, blood was collected by orbital puncture under isoflurane anaesthesia and mice were euthanised by cervical dislocation. Tissues were excised and immediately frozen in liquid nitro- gen followed by storage at $-80^{\circ} \mathrm{C}$. All experiments were blind to group and outcome assessment. All animal experiments were authorised by the animal welfare committee of Wageningen University.

\section{Intraperitoneal glucose tolerance test}

After a $6 \mathrm{~h}$ fast mice were injected intraperitoneally with glucose $(1.2 \mathrm{~g} / \mathrm{kg}$ body weight for mice on chow, $0.8 \mathrm{~g} / \mathrm{kg}$ body weight for mice on HFD). Blood was collected by tail 
bleeding after 0, 15, 30, 60, 90 and 120 min, and glucose was measured using an AccuChek compact (Roche Diagnostics, Almere, the Netherlands).

\section{Intraperitoneal insulin tolerance test}

After a $6 \mathrm{~h}$ fast mice were injected intraperitoneally with insulin $(0.75 \mathrm{U} / \mathrm{kg}$ body weight). Blood was collected by tail bleeding after $0,15,30,45,60$ and $90 \mathrm{~min}$, and glucose was measured using an Accu-Chek compact (Roche Diagnostics).

\section{Histological stainings}

Histological analysis of musculus gastrocnemius morphology and macrophage infiltration was done using hematoxylin and eosin (H\&E) staining. On the day of euthanasia, samples were frozen in isopentane mixed with dry ice and stored at $-80^{\circ} \mathrm{C}$. Tissue was embedded in Tissue-Tek O.C.T. Compound (Sakura Finetek, Alpen aan de Rijn, the Netherlands), sectioned at $10 \mu \mathrm{m}$ in a cryostat chamber, fixed on a Superfrost glassslide, left to dry for $30 \mathrm{~min}$ and stored at $-20^{\circ} \mathrm{C}$. Samples were stained at room temperature in Mayer hematoxylin solution for $10 \mathrm{~min}$ and in eosin $\mathrm{Y}$ solution for 30 sec.

\section{Measurement of plasma variables}

Blood was collected into EDTA-coated tubes. Blood samples were placed on ice and centrifuged at $4^{\circ} \mathrm{C}$ for $10 \mathrm{~min}$ at $10,000 \mathrm{~g}$. Plasma was collected and stored at $-80^{\circ} \mathrm{C}$. To measure plasma MCP-1 concentration a DuoSet ELISA Development kit against mouse MCP-1 was used (R\&D Systems, Minneapolis, MN, USA). For insulin measurements an ultra-sensitive mouse insulin ELISA kit was used (Crystal chemicals, Downers Grove, IL, USA). For the other plasma measurements, the following kits were used: NEFA Reagent set, Triglycerides Liquicolor, Cholesterol Liquicolor (all Instruchemie, Delfzijl, the Netherlands) and Glucose GOD FS (DiaSys, Holzheim, Germany). All measurements were performed according to the manufacturers' protocols.

\section{Affymetrix microarray}

Microarray analysis was performed on RNA of the musculus gastrocnemius. The RNA of 8 WT mice and $8 \mathrm{Tg}$ mice was extracted using TRIzol reagent (Invitrogen, Carlsbad, CA, USA) and a Tissuelyser II (Qiagen, Germantown, MD, USA), purified using the RNeasy Micro Kit (Qiagen) and the integrity were verified with the RNA 6000 Nano assay on the Agilent 2100 Bioanalyzer (Agilent Technologies, Amsterdam, the Netherlands). Hybridization, washing, and scanning of the Affymetrix GeneChip Mouse Gene 1.1 ST Array were performed on Affymetrix GeneTitan. Scans of the Affymetrix 
arrays were processed using packages from the Bioconductor project [26]. Arrays were normalized and raw signal intensities were obtained using the Robust Multiarray Average method $[27,28]$. Probe sets were defined according to Dai et al. [29]. In this method probes are assigned to Entrez IDs as a unique gene identifier. The $\mathrm{P}$ values were calculated using an Intensity-Based Moderated T-statistic (IBMT) [30]. The microarray data were submitted to the Gene Expression Omnibus (accession number pending). Gene set enrichment analysis (GSEA) was used to find enriched gene sets in the induced or suppressed genes [31]. Genes were ranked based on the IBMT-statistic and subsequently analyzed for over- or underrepresentation in predefined gene sets derived from Gene Ontology, KEGG, National Cancer Institute, PFAM, Biocarta, Reactome and WikiPathways pathway databases. Only gene sets consisting of more than 15 and fewer than 500 genes were taken into account. Statistical significance of GSEA results was determined using 1,000 permutation.

\section{RNA isolation and quantitative real-time PCR}

Total RNA was extracted from different mouse tissues using TRIzol reagent (Invitrogen, Bleiswijk, the Netherlands) and a Tissuelyser II (Qiagen, Venlo, the Netherlands). Reverse transcription was performed using Superscript II and oligo(dT) primers (Invitrogen). PCR-amplifications were carried out using iQ SYBR Green Supermix on a CFX384 Touch real-time PCR detection system (Bio-Rad, Hercules, CA, USA). The sequences of the primers used for quantitative real-time PCR (qPCR) are shown in Supplemental Table 1. All genes were normalised to 36b4. Primer efficiencies were determined using LinRegPCR v11.1 (http://LinRegPCR.nl) [32]. Relative expression of the transcript levels was calculated as described previously [33].

\section{Western blotting}

Total protein extracts were obtained from musculus gastrocnemius to detect MCP-1. Tissue was homogenised using a Tissuelyser II (Qiagen) in protein extraction buffer without bromophenol blue ( $62.5 \mathrm{mmol} / 1 \mathrm{Tris} \mathrm{HCl}, \mathrm{pH} 6.8,2 \% \mathrm{SDS}, 10 \%$ glycerol and $10 \mathrm{mmol} / \mathrm{l}$ dithiothreitol) and protein concentration was measured using Bradford protein assay. Protein $(50 \mu \mathrm{g})$ was boiled, subjected to SDS- PAGE and proteins transferred to nitrocellulose membrane (Immobilon; Millipore, Billerica, MA, USA). Membranes were blocked and incubated with anti-MCP-1 (R17; Santa Cruz, Dallas, TX, USA) and anti-Actin (Sigma Aldrich, St Louis, MO, USA). To obtain total protein extracts from musculus gastrocnemius to detect phospho-Akt, tissue was homogenised using a Tissuelyser II (Qiagen) in protein extraction buffer with phosphatase inhibitors (50 mmol/1 Tris HCl, pH 4.7, 1 mmol/1 EDTA, 150 mmol/1 NaCl, 1\% NP40, 0.25\% 
sodium deoxycholate, $2 \mathrm{mmol} / \mathrm{l}$ sodium orthovanadate and $5 \mathrm{mmol} / \mathrm{l}$ sodium fluoride, containing freshly added protease inhibitor cocktail [Roche Applied Science, Penzberg, Germany]). After being centrifuged at $20,800 \mathrm{~g}$ for $10 \mathrm{~min}$ at $4^{\circ} \mathrm{C}$, supernatant fractions were collected and $50 \mu \mathrm{g}$ of protein was boiled with Laemmli sample buffer. The protein was subjected to SDS-PAGE and transferred to a polyvinylidene difluoride membrane (Immobilon; Millipore). Membranes were blocked and incubated with anti-Akt (in house), anti-phospho-Akt (Ser473) (Cell Signalling Technology, Danvers, MA, USA) and anti-Actin (Sigma Aldrich). Quantification was carried out using ImageJ $1.49 \mathrm{~m}$ (http://imagej.nih.gov).

\section{Statistical analysis}

For statistical analysis Student's $t$ test was used. Data are means \pm SEM and $p<0.05$ is considered statistically significant. One mouse from the WT group, fed HFD, was excluded from subsequent analysis because it was mistakenly injected with a higher dose of insulin during the insulin tolerance test. Some qPCR data on gene expression were excluded from the results section because the genes were expressed at a low level, with $\mathrm{Ct}$ values greater than 32 in both the WT and Mcp-1-Tg mice. 


\section{Results}

Skeletal muscle-specific overexpression of MCP-1 results in a local inflammatory response

To assess the impact of muscle-specific overexpression of MCP-1 on whole-body glucose homeostasis and insulin sensitivity, we used Tg mice that overexpress Mcp-1 under the control of the Mck promotor [25].
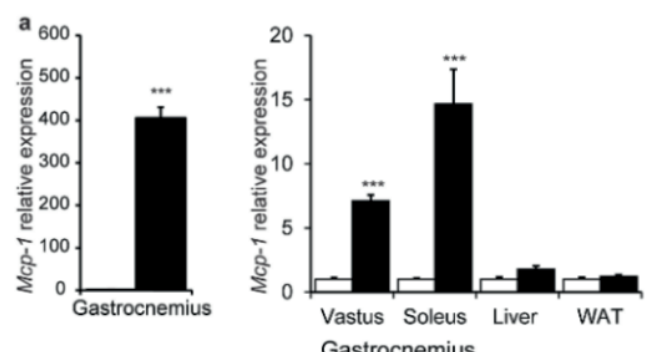

b

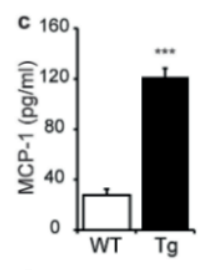

d
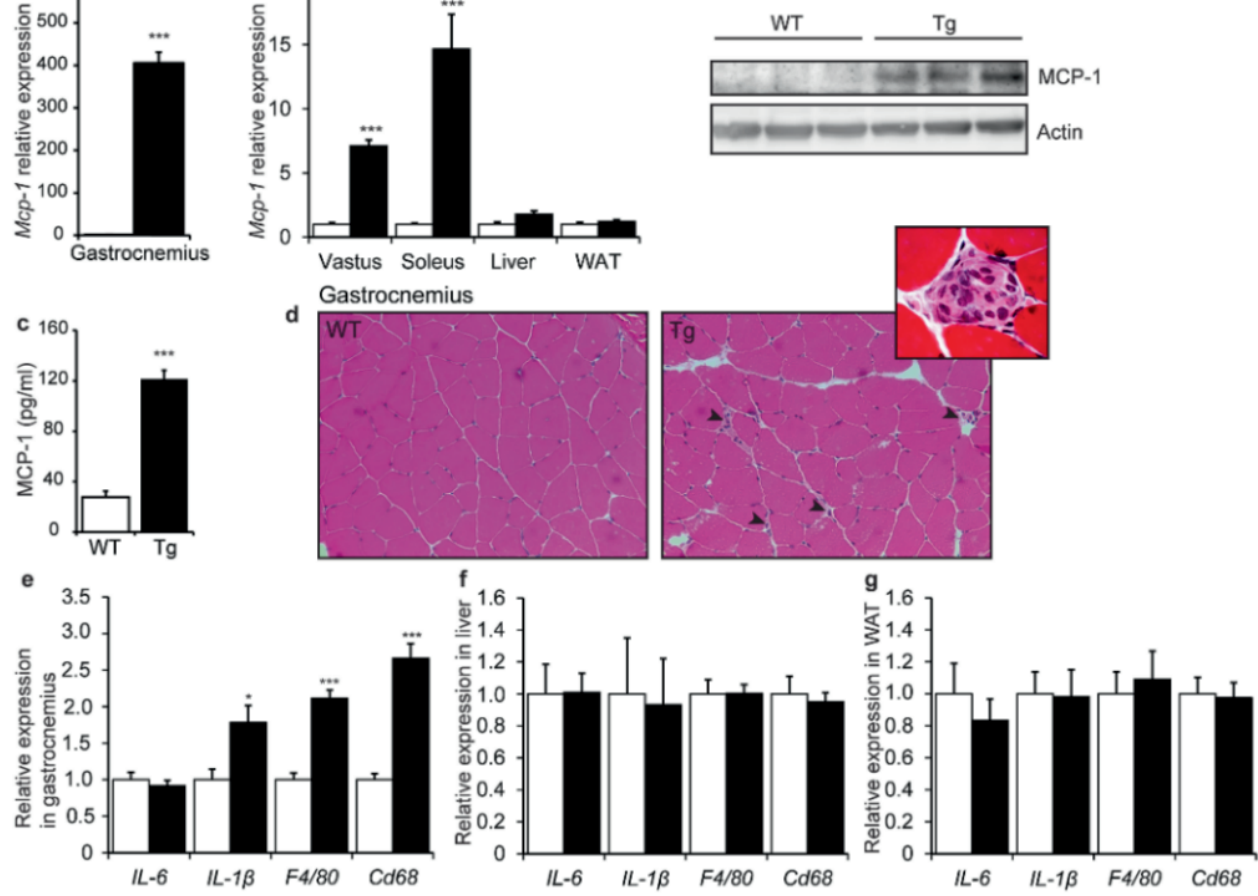

Figure 1: Skeletal muscle-specific overexpression of MCP-1 provokes inflammation. (a) Relative gene expression of $M c p-1$ measured by qPCR; $n=8$ (WT mice) or $n=11$ (Mcp-1-Tg mice). (b) Representative western blot analysis of MCP-1 overexpression in Mcp-1-Tg mice. (c) Plasma concentration of MCP-1; $\mathrm{n}=8$ (WT mice) or $\mathrm{n}=11$ (Mcp- 1 -Tg mice). (d) H\&E staining of representative sections of musculus gastrocnemius of WT and Mcp-1-Tg mice (magnification $\times 200$ ). Arrowheads indicate macrophage infiltration. Insert: H\&E staining of musculus vastus (magnification $\times 1,000$ ), showing immune cell infiltrate. $\mathbf{e}-\mathbf{g}$ : Relative gene expression of Il6, $I l l b, F 4 / 80$ and $C d 68$ in gastrocnemius (e), liver (f) and WAT (g) measured by qPCR; $n=8$ (WT mice) or $n=11$ (Mcp-1-Tg mice). White bars, WT mice; black bars, Mcp-1-Tg mice. Data are means \pm SEM; ${ }^{*} p<0.05$ and ${ }^{* * *} p<0.001$ according to Student's $t$ test for WT vs Mcp-1-Tg mice 
The marked overexpression of $M c p-1$ in the transgenic mice was confirmed at the mRNA level in the musculus gastrocnemius, vastus and soleus, whereas $M c p-1$ was only weakly induced or not induced in the liver and white adipose tissue (WAT), respectively (Figure 1a). Since the overexpression of $M c p-1$ was most pronounced in the gastrocnemius muscle, we used the gastrocnemius in all follow-up experiments. Overexpression of $M c p-1$ in the gastrocnemius of the $M c p-1-\mathrm{Tg}$ mice was confirmed at the protein level (Figure 1b). Furthermore, the plasma concentration of MCP-1 in the Mcp-1-Tg mice was increased approximately 4.4-fold compared with WT mice, indicating that MCP-1 is secreted from skeletal muscle (Figure 1c). H\&E staining indicated enhanced inflammation in the gastrocnemius of the Mcp-1-Tg mice as compared with WT mice, as illustrated by infiltration of macrophages between the muscle fibres (Figure 1d). In addition, mRNA levels of $I l l b$ and the macrophage markers $F 4 / 80$ and $C d 68$ were significantly increased in the gastrocnemius of the $\mathrm{Mcp}$ 1 -Tg mice (Figure 1e). In contrast, expression of the same inflammatory markers in liver (Figure 1f) and WAT (Figure 1g) did not reveal any differences between WT and Mcp1-Tg mice.

Next, we performed microarray analysis on the gastrocnemius of eight WT mice and eight Mcp-1-Tg mice to study the overall effect of MCP-1 overexpression on gene expression in skeletal muscle. Overexpression of MCP-1 caused a clear inflammatory gene expression signature (Figure 2a). The 25 most highly induced genes were all involved in inflammation and/or immunity, including the genes encoding lysozyme ( $L y z 1$ ), the immune cells surface markers $C d 52$ and $C d 180$, and the secreted protein Retnla. Interestingly, the most highly induced gene was Mcp-1 (99-fold), followed by its receptor $C$ cr2. Gene set enrichment analysis (GSEA) corroborated the pronounced induction of inflammation and immune-related pathways. Specifically, the top 20 of most highly enriched pathways are all linked to inflammation, including chemokine signalling, T cell receptor signalling, phagocytosis and inflammatory disease-related pathways (Figure $2 b$ ). The complete list of significantly regulated genes (q value $<0.05$ ) is presented in Supplemental Table 2. Taken together, these data indicate that MCP-1 overexpression promotes inflammation in skeletal muscle. 
a

\begin{tabular}{|c|c|c|c|c|c|c|c|c|c|c|c|c|c|c|c|c|c|c|c|}
\hline \multirow[b]{2}{*}{ Gene name } & \multicolumn{8}{|c|}{ WT } & \multicolumn{8}{|c|}{$\mathrm{Tg}$} & \multirow{2}{*}{$\begin{array}{l}\text { Mean } \\
\text { FC }\end{array}$} & \multirow{2}{*}{ p-Value } & \\
\hline & 1 & 2 & 3 & 4 & 5 & 6 & 7 & 8 & 1 & 2 & 3 & 4 & 5 & 6 & 7 & 8 & & & \\
\hline Mcp-1/Ccl2 & & & & & & & & & & & & & & & & & 99.1 & 0 & -5 \\
\hline Ccr2 & & & & & & & & & & & & & & & & & 6.9 & $2.53 \times 10^{-14}$ & \\
\hline Lyz1 & & & & & & & & & & & & & & & & & 5.8 & $1.58 \times 10^{-13}$ & \\
\hline Retnla & & & & & & & & & & & & & & & & & 5.7 & $9.02 \times 10^{-11}$ & \\
\hline$H 2-A b 1$ & & & & & & & & & & & & & & & & & 5.3 & $4.22 \times 10^{-14}$ & \\
\hline Ear2 & & & & & & & & & & & & & & & & & 5.1 & $1.03 \times 10^{-07}$ & \\
\hline $\mathrm{H} 2-\mathrm{Aa}$ & & & & & & & & & & & & & & & & & 4.9 & $1.27 \times 10^{-12}$ & \\
\hline Cd74 & & & & & & & & & & & & & & & & & 4.8 & $8.66 \times 10^{-14}$ & \\
\hline$H_{2}-E b 1$ & & & & & & & & & & & & & & & & & 4.7 & $4.24 \times 10^{-12}$ & \\
\hline Cd52 & & & & & & & & & & & & & & & & & 4.3 & $7.84 \times 10^{-12}$ & \\
\hline Ndnf & & & & & & & & & & & & & & & & & 4.2 & $4.97 \times 10^{-13}$ & 5 \\
\hline Fcrls & & & & & & & & & & & & & & & & & 3.9 & $2.16 \times 10^{-10}$ & \\
\hline Cd180 & & & & & & & & & & & & & & & & & 3.8 & $2.49 \times 10^{-13}$ & \\
\hline Slamf9 & & & & & & & & & & & & & & & & & 3.8 & $3.16 \times 10^{-12}$ & \\
\hline Mpeg1 & & & & & & & & & & & & & & & & & 3.6 & $1.99 \times 10^{-10}$ & \\
\hline H2-DMb1 & & & & & & & & & & & & & & & & & 3.4 & $2.48 \times 10^{-05}$ & \\
\hline H2-DMa & & & & & & & & & & & & & & & & & 3.4 & $1.68 \times 10^{-11}$ & \\
\hline Ly86 & & & & & & & & & & & & & & & & & 3.3 & $5.99 \times 10^{-09}$ & \\
\hline Csf2ra & & & & & & & & & & & & & & & & & 3.2 & $9.79 \times 10^{-11}$ & \\
\hline Laptm5 & & & & & & & & & & & & & & & & & 3.2 & $1.39 \times 10^{-12}$ & \\
\hline Alcam & & & & & & & & & & & & & & & & & 3.2 & $3.41 \times 10^{-06}$ & \\
\hline Atp8b4 & & & & & & & & & & & & & & & & & 3.2 & $3.95 \times 10^{-10}$ & \\
\hline Ptprc & & & & & & & & & & & & & & & & & 3.1 & $6.46 \times 10^{-11}$ & \\
\hline Lyz2 & & & & & & & & & & & & & & & & & 3.1 & $6.49 \times 10^{-09}$ & \\
\hline Pilra & & & & & & & & & & & & & & & & & 3.0 & $2.06 \times 10^{-10}$ & \\
\hline
\end{tabular}

b

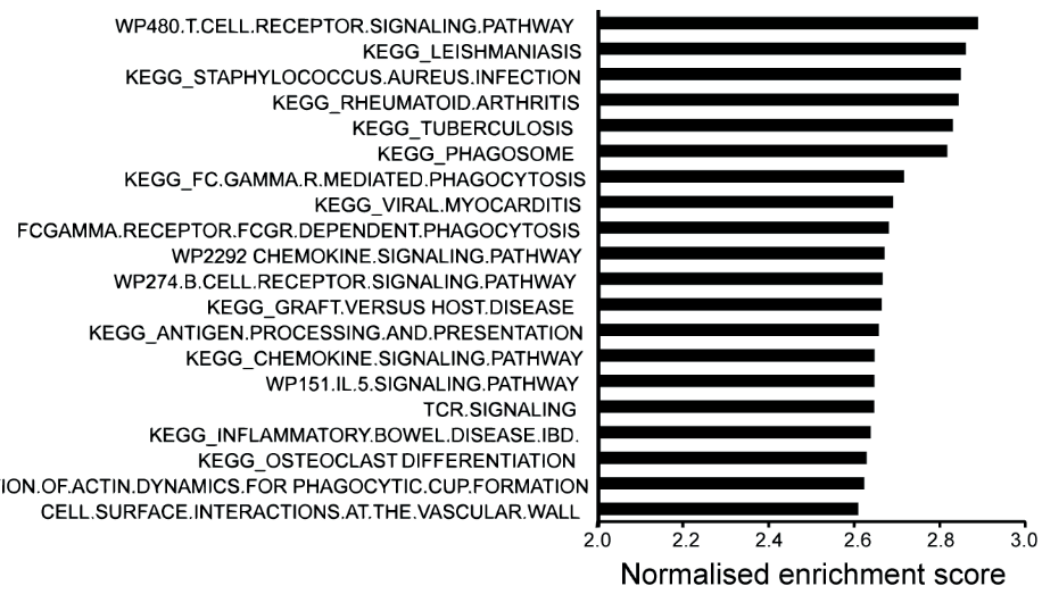

Figure 2: MCP-1 overexpression induces an inflammatory gene expression profile. (a) Gene expression changes in gastrocnemius muscle, illustrated by a heatmap of the 25 most highly induced genes in Mcp-1-Tg mice compared with WT mice. FC, fold change. (b) Gene sets positively enriched in the gastrocnemius of $M c p-1-\mathrm{Tg}$ mice ranked according to normalised enrichment score (GSEA). The enrichment score reflects the degree to which a gene set is overrepresented at the top or bottom of a ranked list of genes. Normalisation accounts for differences in gene set size and in correlations between gene sets and the expression dataset. 


\section{No metabolic phenotype can be observed in mice overexpressing MCP-1 in skeletal muscle when fed a normal chow diet}

To test whether skeletal muscle-specific overexpression of MCP-1 and associated inflammation may also influence glucose homeostasis and insulin sensitivity, Mcp-1$\mathrm{Tg}$ mice and their WT littermates were subjected to detailed metabolic characterisation. Mcp-1-Tg and WT mice had similar weights of musculus gastrocnemius, musculus vastus, liver and WAT (Figure 3a).
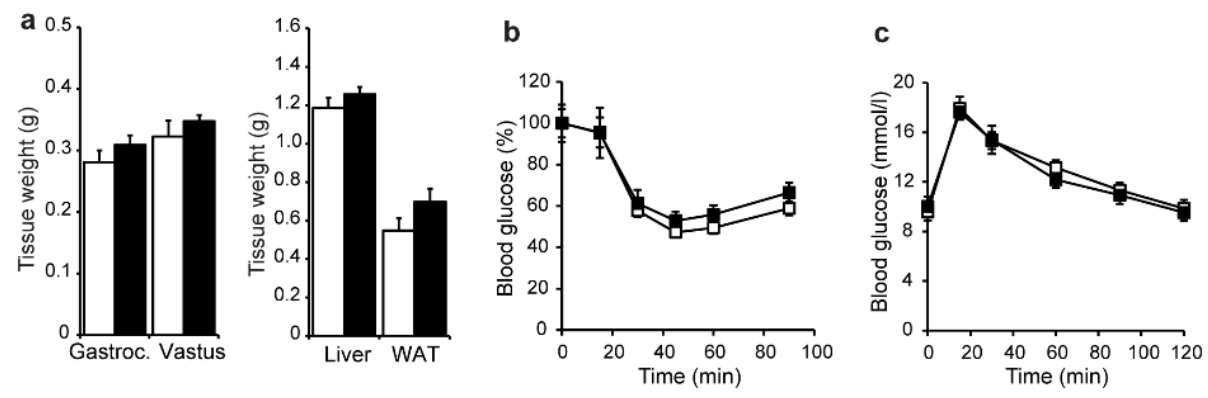

d
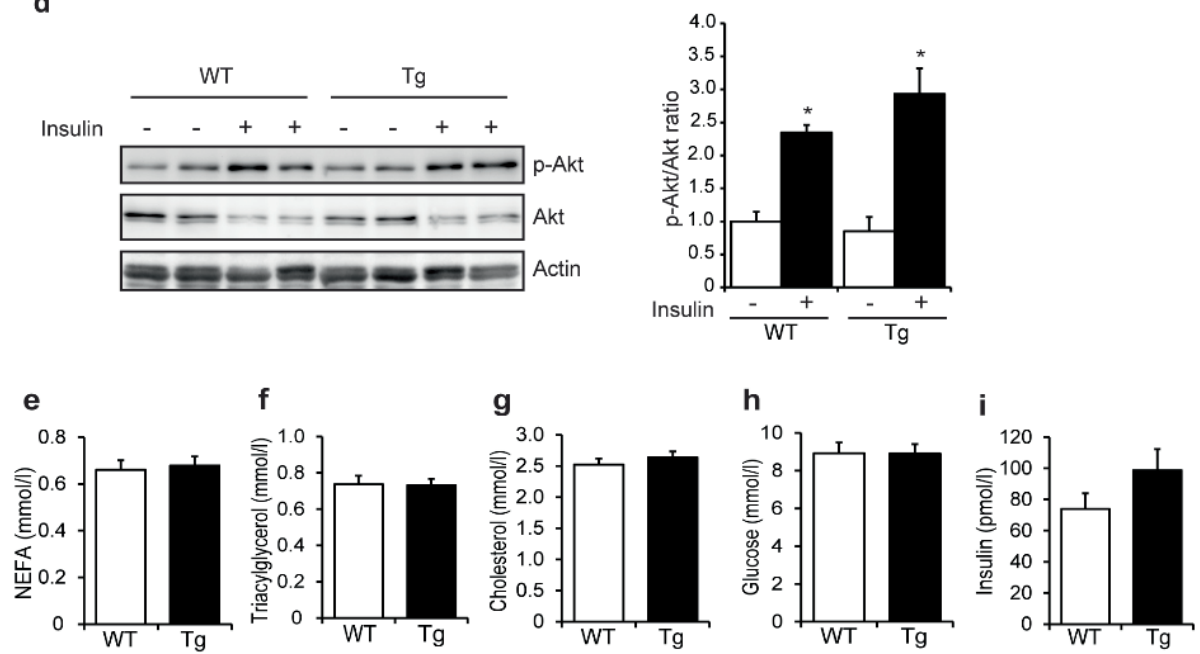

Figure 3: No metabolic phenotype is observed in mice overexpressing $M c p-1$ in skeletal muscle under normal chow diet. (a) Tissue weights of musculus gastrocnemius (Gastroc.), musculus vastus, liver and WAT; $n=8$ (WT mice) or $n=11$ (Mcp- 1 -Tg mice). (b, c) Blood glucose level during insulin tolerance test (b) and glucose tolerance test (c); $n=8$ (WT and Mcp-1-Tg mice). (d) Representative western blot showing phosphorylation levels of Akt in gastrocnemius of WT and Mcp-1-Tg mice upon insulin injection. Bar graph shows quantification of $\mathrm{p}$-Akt/Akt ratio performed in four mice per treatment group and normalised to WT mice without insulin. (e- 
i) Plasma concentration of NEFA (e), triacylglycerol (f), cholesterol (g), glucose (h) and insulin (i); $n=8$ (WT mice) or $n=11$ (Mcp-1-Tg mice). White bars and squares, WT mice; black bars and squares, Mcp-1-Tg mice. Data are means \pm SEM; ${ }^{*} p<0.05$ according to Student's $t$ test for insulin-treated mice vs control-treated mice (d).

Intriguingly, the results of an intraperitoneal insulin tolerance test (Figure 3b) and intraperitoneal glucose tolerance test (Figure 3c) did not differ between $M c p-1-\mathrm{Tg}$ and WT mice. In addition, we analysed the effect of muscle-specific MCP-1 overexpression on the sensitivity of muscle to i.p. injection of insulin, using phosphorylation of Akt as a readout. Insulin injection markedly stimulated Akt phosphorylation in the gastrocnemius muscle (Figure 3d). However, no significant difference in phospho-Akt could be observed between WT and Mcp-1-Tg mice, indicating that MCP-1 overexpression does not alter insulin-dependent signalling in skeletal muscle. Finally, the Mcp-1-Tg mice did not show any changes in plasma concentrations of NEFA, triacylglycerol, cholesterol, glucose or insulin when compared with WT mice (Figure $3 \mathrm{e}-\mathrm{i}$ ). Taken together, our results indicate a lack of an effect of muscle-specific MCP-1 overexpression on insulin sensitivity and glucose homeostasis in mice fed a normal chow diet.

\section{HFD feeding does not result in metabolic alterations in muscle-specific Mcp-1-Tg mice}

One possible explanation for the lack of effect of MCP-1 overexpression on variables associated with insulin sensitivity is related to the fact that the mice were fed normal chow and were therefore lean. To determine the impact of muscle-specific overexpression of MCP-1 on glucose homeostasis and insulin sensitivity in the context of obesity and insulin resistance, muscle-specific $M c p-1$-Tg and WT littermates were rendered obese and insulin resistant by chronic high-fat feeding. The muscle- specific overexpression of Mcp-1 in the Mcp-1-Tg mice was maintained after HFD (Figure 4a), accompanied by a 10.9-fold increase in plasma MCP-1 levels (Figure 4b) . H\&E staining showed more pronounced macrophage infiltration in the gastrocnemius of the Mcp-1$\mathrm{Tg}$ mice compared with WT mice (Figure 4c). In addition, mRNA levels of $I l l b$ and the macrophage markers $F 4 / 80$ and $C d 68$ were significantly increased in the gastrocnemius of the Mcp-1-Tg mice (Figure 4d). In contrast, expression of inflammatory markers remained unchanged in liver and adipose tissue of the Mcp-1-Tg mice, with the exception of macrophage marker $F 4 / 80$ and alternatively-activated macrophage marker $C d 206$, which were both significantly reduced in liver of $M c p-1$-Tg mice (Supplemental Figure 1). 


\section{a}

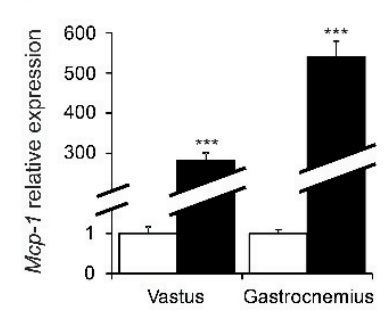

c

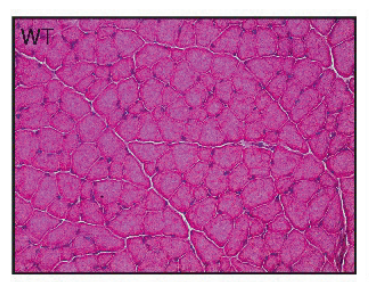

e

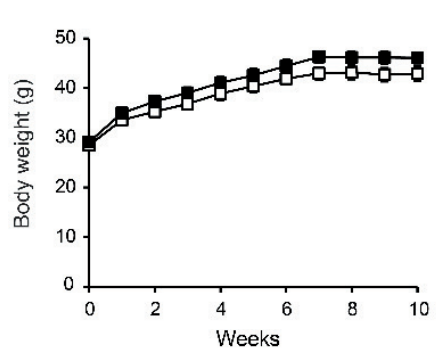

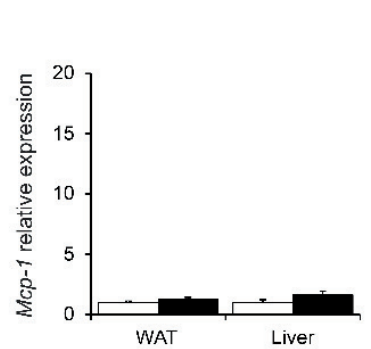

b

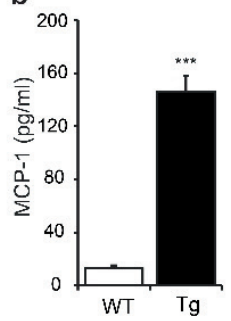

d
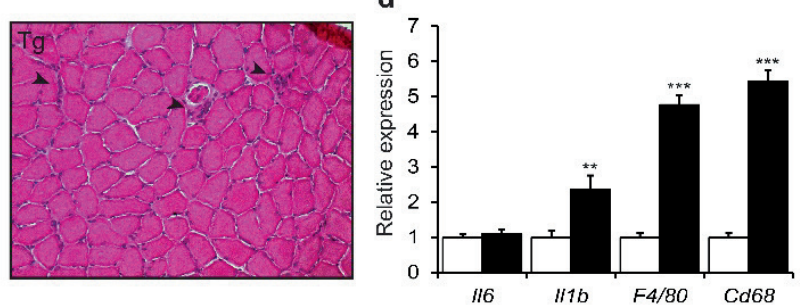

f
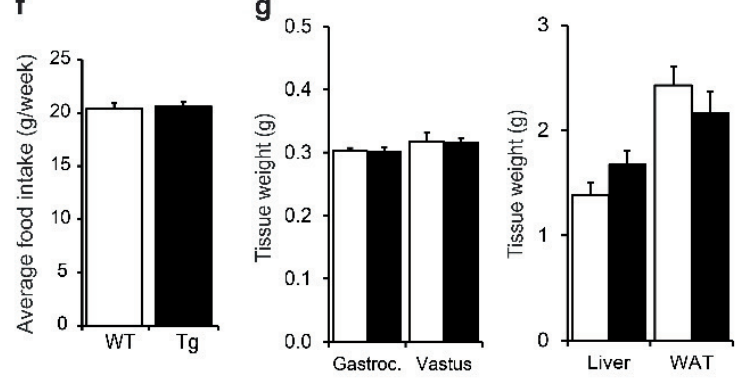

Figure 4: Characterisation of muscle-specific Mcp-1-Tg mice fed an HFD. (a) Relative gene expression of Mcp-1 measured by qPCR. (b) Blood concentration of MCP-1. (c) H\&E staining of representative sections of gastrocnemius of WT and Mcp-1-Tg mice (magnification $\times 200$ ); Arrowheads indicate macrophage infiltration. (d) Relative gene expression of $I l 6, I l 1 b, F 4 / 80$ and Cd68 measured by qPCR. (e): Body weight changes in the WT and Mcp-1-Tg mice. (f) Average food intake from week 2 to week 8. (g) Tissue weights of musculus gastrocnemius (Gastroc.), musculus vastus, liver and WAT. White bars and squares, WT mice; black bars and squares, $M c p$ 1 -Tg mice. Data are means \pm SEM; $n=11$ (WT mice) or $n=12$ (Mcp- 1 -Tg mice); ${ }^{*} p<0.01$ and *** $p<0.001$ according to Student's t test for WT vs Mcp-1-Tg mice.

Whole-body weight (Figure 4e) and food intake (Figure 4f) did not differ between $M c p$ 1-Tg mice and WT mice on HFD, and there was no difference in weights of the musculus gastrocnemius, musculus vastus, liver and WAT (Figure 4g). An intraperitoneal insulin tolerance test and glucose tolerance test revealed decreased glucose tolerance in mice fed HFD compared with chow. 


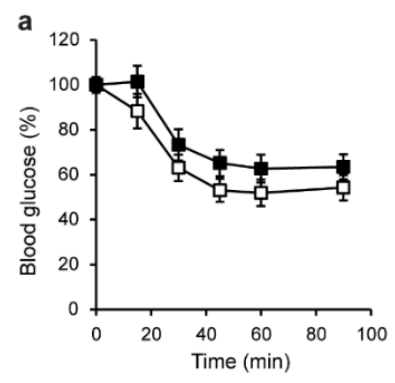

C

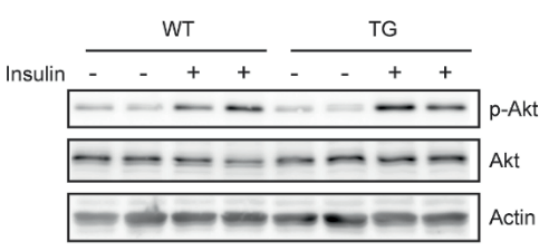

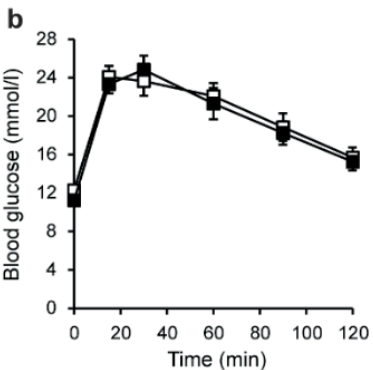
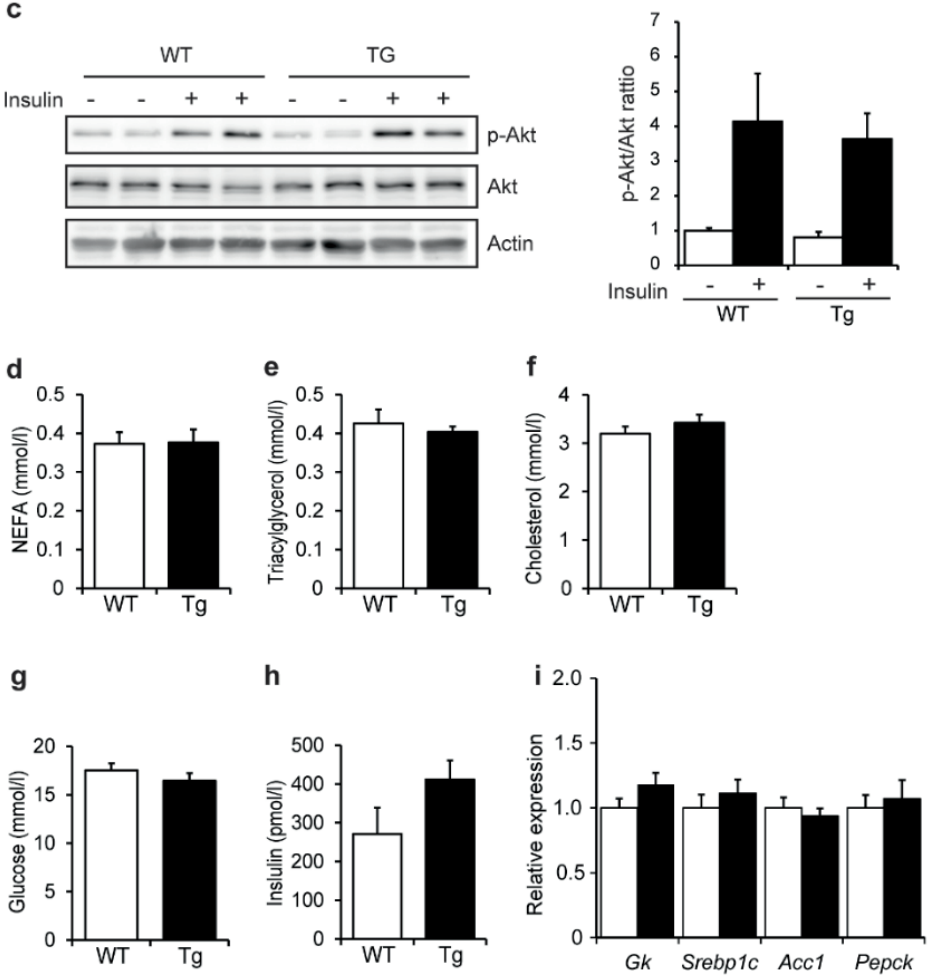

Figure 5: HFD feeding does not result in metabolic alterations in muscle- specific Mcp-1-Tg mice. (a, b) Plasma glucose level during insulin tolerance test (a) and glucose tolerance test (b); $n=11$ (WT mice) or $n=12$ (Mcp-1-Tg mice). (c) Representative western blot showing phosphorylation levels of Akt in gastrocnemius of WT and Mcp-1-Tg mice upon injection of insulin. Bar graph shows quantification of $\mathrm{p}$-Akt/Akt ratio performed on two mice per treatment group and normalised to WT mice without insulin. (d-h) Plasma concentration of NEFA (d), triacylglycerol (e), cholesterol (f), glucose (g) and insulin (h); $n=7$ (WT mice) or $n=8$ (Mcp-1-Tg mice). (i) Relative gene expression of Gk, Srebplc, Accl and Pepck in liver measured by qPCR; $n=12$ (for WT and Mcp-1-Tg mice). White bars and squares, WT mice; black bars and squares, Mcp-1-Tg mice. Data are means \pm SEM; $\uparrow p=0.08, \ddagger p=0.03$ according to Student's $t$ test for insulintreated mice vs control-treated mice $(\mathbf{c})$. 
However, no differences in insulin and glucose tolerance were detected when comparing diet-induced obese $M c p-1-\mathrm{Tg}$ mice with WT mice (Figure 5a, b). As observed in chow-fed mice, insulin-induced Akt phosphorylation was similar in the gastrocnemius of HFD-fed Mcp-1-Tg mice compared with HFD-fed WT mice (Figure $5 c)$. In addition, none of the plasma metabolites measured was significantly changed in the Mcp-1-Tg mice compared with WT mice. (Figure 5d-h). Finally, hepatic expression of insulin-sensitive genes Gk, Srebplc (also known as Srebfl), Accl (Acaca), and Pepck (Pckl) was unaffected by muscle-specific MCP-1 over-expression (Figure 5i). Taken together, muscle-specific overexpression of MCP-1 did not affect insulin sensitivity and glucose homeostasis in obese insulin-resistant mice. 


\section{Discussion}

The aim of the present study was to assess to what extent elevation of MCP-1 production in muscle and elevated MCP-1 levels in plasma interfere with insulin signalling in skeletal muscle and promote insulin resistance. To this end, an Mck-Mcp-1-Tg mouse model that specifically overexpresses MCP-1 in skeletal muscle was used. We show that muscle-specific overexpression of MCP-1 promotes inflammation in skeletal muscle but does not influence insulin signalling in skeletal muscle and has no effect on whole-body insulin sensitivity and glucose tolerance in either lean or obese mice. These findings therefore indicate that muscle inflammation by itself is not sufficient to evoke whole-body insulin resistance.

Studies to date have not been consistent in establishing a causal relation between muscle-specific stimulation or repression of inflammatory molecules and alterations in insulin sensitivity. On the one hand, it has been shown that over- production of constitutively activated c-Jun N-terminal kinase in skeletal muscle impairs insulin signalling in mice by modulating serine and threonine phosphorylation of Akt and IRS1 [34]. On the other hand, muscle-specific ablation of IкB kinase 2, resulting in reduced activation of the transcription factor nuclear factor- $\mathrm{KB}(\mathrm{NF}-\kappa \mathrm{B})$, had no effect on obesity-induced insulin resistance and on insulin signalling in muscle [35]. Similarly, muscle-specific ablation of signal transducer and activator of transcription 3 (STAT3), another transcription factor activated by proinflammatory cytokines, showed no effect on obesity-induced insulin resistance, as assessed by glucose tolerance test, clamp studies and measurement of 2-deoxyglucose uptake [36]. Our study indicates that local activation of inflammation in muscle via MCP-1 overexpression does not affect insulin sensitivity and insulin action. Along the same lines, overexpression of NF- $\mathrm{KB}$ subunit p65 and IкBK $\beta$ in single muscles of rats using in vivo electrotransfer had no effect on glucose disposal in muscle under hyperinsulinaemic-euglycaemic clamp conditions, suggesting that activation of the IкBK $\beta-\mathrm{NF}-\kappa \mathrm{B}$ pathway in muscle does not seem to be an important local mediator of insulin resistance [37].

Presently, it is difficult to merge the diverse findings into a coherent and consistent picture describing the role and impact of inflammatory signalling in muscle on local insulin resistance. A provocative conclusion emerging from our study and other 'negative' studies is that obesity-induced insulin resistance in muscle, the primary organ accounting for insulin-mediated glucose disposal, is not dependent on inflammatory signalling in muscle itself; this in turn can be extrapolated to suggest that development of insulin resistance in muscle during obesity does not require inflammatory mediators 
that affect skeletal muscle. In future studies, more attention should be accorded to clarification of the role of inflammatory signalling from adipose tissue to muscle in the development of insulin resistance.

In contrast to our findings, Patsouris et al observed modest whole-body and muscle insulin resistance and mildly elevated plasma glucose levels in Mck-Mcp-1-Tg mice [25]. One of the potential reasons for the discrepancy between our data and those of Patsouris is the composition of the background diet, as our chow diet and HFD are different from the diets used by Patsouris et al. Another important difference between our study and the study by Patsouris and colleagues is that we measured plasma metabolic variables (i.e. insulin, glucose, triacylglycerol) in $6 \mathrm{~h}$-fasted mice, whereas Patsouris assessed the same variables in fed mice. Unfortunately, additional relevant experimental details (number of mice used, sex of the mice, time of euthanasia) are not mentioned in the Patsouris paper, which would have allowed for a more detailed comparison between the two studies [25].

In contrast to the present study in mice with muscle-specific overexpression of MCP1, studies performed in mice with adipose tissue-specific overexpression of MCP-1 or expression of a dominant-negative mutant of MCP-1 show that adipose tissue-derived MCP-1 reduces glucose tolerance and insulin sensitivity and blunts insulin-mediated signalling in skeletal muscle $[11,12]$. Also, in vitro treatment of human skeletal muscle cells or the $\mathrm{C} 2 \mathrm{C} 12$ muscle cell line with recombinant MCP-1 protein results in decreased Akt phosphorylation upon insulin stimulation $[10,11]$. This raises the question why MCP-1 secreted from adipose tissue is able to alter whole-body and muscle insulin sensitivity whereas MCP-1 secreted from the muscle is not, especially since the levels of plasma MCP-1 were comparable in both transgenic mouse models [11, 12]. One explanation may be that circulating MCP-1 only plays a minor role in regulating wholebody insulin sensitivity and that local MCP-1 concentrations predominantly determine the metabolic outcome: raising MCP-1 levels in muscle only results in local inflammation, while increased MCP-1 expression in adipose tissue starts a cascade of events including altered adipose-resident immune cell profiles and altered output of adipokines and metabolites, ultimately resulting in impaired whole-body insulin sensitivity. It should be noted, however, that acute or chronic elevation of circulating MCP-1 by injection results in insulin resistance [38]. Accordingly, a possible alternative explanation is that circulating MCP-1 is able to affect insulin sensitivity but that the form secreted by adipose tissue is different from the form secreted by muscle tissue. It is known that MCP-1 exists in an unglycosylated (8-12 $\mathrm{kDa}$ ) and in a glycosylated form $(17-30 \mathrm{kDa})$ [39-41] and it has been suggested that the glycosylated form can bind to 
cell membranes, increasing the local MCP-1 expression, while unglycosylated MCP-1 is more easily diffusible and able to form a potent chemotactic gradient [42]. The MCP1 protein detected by western blotting in this study has a molecular mass of approximately $18 \mathrm{kDa}$, which suggests that the MCP-1 expressed in skeletal muscle is glycosylated. Furthermore, in vitro treatment of muscle cells with MCP-1 and acute or chronic elevation of MCP-1 by injection in vivo, which both resulted in insulin resistance, were all performed with unglycosylated recombinant MCP-1 protein produced in E. coli $[10,11,38]$. More research is therefore needed to investigate the glycosylation status of MCP-1 originating from different tissues. Moreover, it would be interesting to determine whether MCP-1 glycosylation status influences insulin signalling in insulin-sensitive tissues.

By acting as a myokine and as an inflammatory mediator, MCP-1 displays characteristics similar to those of IL-6, the most studied and discussed myokine (reviewed in [22]). It has been suggested that IL-6 produced by skeletal muscle has a stimulatory influence on insulin sensitivity in skeletal muscle, whereas opposite effects have been shown for elevated IL-6 levels in adipose tissue and liver [23,43-45]. Accordingly, the effect of both MCP-1 and IL-6 on insulin sensitivity seems to be very tissue-specific and may also depend on whether they are induced acutely or chronically [22]. Examining the parallels between MCP-1 and IL-6 may help unravel the underlying mechanism accounting for the tissue-specific actions of these two dual-faced regulators of glucose homeostasis.

A limitation of our study is that we only assessed muscle-specific insulin signalling at the level of Akt phosphorylation and did not examine other insulin-sensitive targets or perform clamp studies. Another limitation is that we cannot rule out that (obesityinduced) overexpression of MCP-1 specifically during adulthood has a different effect on insulin action than permanent MCP-1 overexpression in muscle starting during embryonic development. Future studies using inducible $M c p-1$-Tg mice are necessary to clarify this issue.

In conclusion, elevation of MCP-1 production in skeletal muscle and concomitant elevation of plasma MCP-1 lead to enhanced inflammation in skeletal muscle but have no effect on insulin resistance and glucose tolerance in lean and obese mice and do not affect insulin-mediated signalling in skeletal muscle. 
Acknowledgements: We thank D. Patsouris (Université Claude Bernard Lyon 1, France) for donating the $M c k-M c p-1-\mathrm{Tg}$ mice.

Funding: This study was supported by a grant from the Dutch Diabetes Foundation (Grant 2009.60.003).

Disclosure: The authors declare that there is no duality of interest associated with this manuscript. 


\section{References}

[1] Charakida, M., Khan, T., Johnson, W., Finer, N., Woodside, J., Whincup, P.H., et al., 2014. Lifelong patterns of BMI and cardiovascular phenotype in individuals aged 60-64 years in the 1946 British birth cohort study: An epidemiological study. The Lancet Diabetes and Endocrinology 2(8): 648-54.

[2] Kahn, S.E., Hull, R.L., Utzschneider, K.M., 2006. Mechanisms linking obesity to insulin resistance and type 2 diabetes. Nature 444(7121): 840-6.

[3] Ng, M., Fleming, T., Robinson, M., Thomson, B., Graetz, N., Margono, C., et al., 2014. Global, regional, and national prevalence of overweight and obesity in children and adults during 1980-2013: A systematic analysis for the Global Burden of Disease Study 2013. The Lancet 384(9945): 766-81.

[4] Spiegelman, B.M., Flier, J.S., 2001. Obesity and the regulation of energy balance. Cell 104(4): 531-43.

[5] Donath, M.Y., 2014. Targeting inflammation in the treatment of type 2 diabetes: Time to start. Nature Reviews Drug Discovery 13(6): 465-76.

[6] Hotamisligil, G.S., 2010. Endoplasmic Reticulum Stress and the Inflammatory Basis of Metabolic Disease. Cell 140(6): 900-17.

[7] Skurk, T., Alberti-Huber, C., Herder, C., Hauner, H., 2007. Relationship between adipocyte size and adipokine expression and secretion. Journal of Clinical Endocrinology and Metabolism 92(3): 1023-33.

[8] Ye, J., 2009. Emerging role of adipose tissue hypoxia in obesity and insulin resistance. International Journal of Obesity 33(1): 54-66.

[9] Cinti, S., Mitchell, G., Barbatelli, G., Murano, I., Ceresi, E., Faloia, E., et al., 2005. Adipocyte death defines macrophage localization and function in adipose tissue of obese mice and humans. Journal of Lipid Research 46(11): 2347-55.

[10] Sell, H., Dietze-Schroeder, D., Kaiser, U., Eckel, J., 2006. Monocyte chemotactic protein- 1 is a potential player in the negative cross-talk between adipose tissue and skeletal muscle. Endocrinology 147(5): 2458-67.

[11] Kamei, N., Tobe, K., Suzuki, R., Ohsugi, M., Watanabe, T., Kubota, N., et al., 2006. Overexpression of monocyte chemoattractant protein-1 in adipose tissues causes macrophage recruitment and insulin resistance. Journal of Biological Chemistry 281(36): 26602-14.

[12] Kanda, H., Tateya, S., Tamori, Y., Kotani, K., Hiasa, K.I., Kitazawa, R., et al., 2006. MCP-1 contributes to macrophage infiltration into adipose tissue, insulin resistance, and hepatic steatosis in obesity. Journal of Clinical Investigation 116(6): 1494-505.

[13] Catoire, M., Mensink, M., Kalkhoven, E., Schrauwen, P., Kersten, S., 2014. Identification of human exercise-induced myokines using secretome analysis. Physiological Genomics 46(7): 256-67. 
[14] Mathers, J.L., Farnfield, M.M., Garnham, A.P., Caldow, M.K., Cameron-Smith, D., Peake, J.M., 2012. Early inflammatory and myogenic responses to resistance exercise in the elderly. Muscle and Nerve 46(3): 407-12.

[15] Suzuki, K., Nakaji, S., Yamada, M., Liu, Q., Kurakake, S., Okamura, N., et al., 2003. Impact of a competitive marathon race on systemic cytokine and neutrophil responses. Medicine and Science in Sports and Exercise 35(2): 348-55.

[16] Tantiwong, P., Shanmugasundaram, K., Monroy, A., Ghosh, S., Li, M., DeFronzo, R.A., et al., 2010. NF- $\mathrm{BB}$ activity in muscle from obese and type 2 diabetic subjects under basal and exercise-stimulated conditions. American Journal of PhysiologyEndocrinology and Metabolism 299(5): E794-801.

[17] Vella, L., Caldow, M.K., Larsen, A.E., Tassoni, D., Della Gatta, P.A., Gran, P., et al., 2012. Resistance exercise increases NF- B activity in human skeletal muscle. AJP: Regulatory, Integrative and Comparative Physiology 302(6): R667-73.

[18] Lu, H., Huang, D., Ransohoff, R.M., Zhou, L., 2011. Acute skeletal muscle injury: CCL2 expression by both monocytes and injured muscle is required for repair. The FASEB Journal 25(10): 3344-55.

[19] Yahiaoui, L., Gvozdic, D., Danialou, G., Mack, M., Petrof, B.J., 2008. CC family chemokines directly regulate myoblast responses to skeletal muscle injury. Journal of Physiology 586(16): 3991-4004.

[20] Leggate, M., Carter, W.G., Evans, M.J.C., Vennard, R.A., Sribala-Sundaram, S., Nimmo, M.A., 2012. Determination of inflammatory and prominent proteomic changes in plasma and adipose tissue after high-intensity intermittent training in overweight and obese males. Journal of Applied Physiology 112(8): 1353-60.

[21] Many, G., Hurtado, M.-E., Tanner, C., Houmard, J., Gordish-Dressman, H., Park, J.-J., et al., 2013. Moderate-Intensity Aerobic Training Program Improves Insulin Sensitivity and Inflammatory Markers in a Pilot Study of Morbidly Obese Minority Teens. Pediatric Exercise Science 25(1): 12-26.

[22] Catoire, M., Kersten, S., 2015. The search for exercise factors in humans. FASEB Journal 29(5): 1615-28.

[23] Lambernd, S., Taube, A., Schober, A., Platzbecker, B., Görgens, S.W., Schlich, R., et al., 2012. Contractile activity of human skeletal muscle cells prevents insulin resistance by inhibiting pro-inflammatory signalling pathways. Diabetologia 55(4): $1128-39$.

[24] Harder-Lauridsen, N.M., Krogh-Madsen, R., Holst, J.J., Plomgaard, P., Leick, L., Pedersen, B.K., et al., 2014. Effect of IL-6 on the insulin sensitivity in patients with type 2 diabetes. American Journal of Physiology-Endocrinology and Metabolism 306(7): E769-78.

[25] Patsouris, D., Cao, J.-J., Vial, G., Bravard, A., Lefai, E., Durand, A., et al., 2014. Insulin Resistance is Associated with MCP1-Mediated Macrophage Accumulation in Skeletal Muscle in Mice and Humans. PLoS ONE 9(10): e110653. 
[26] Gentleman, R.C., Carey, V.J., Bates, D.J., Bolstad, B.M., Dettling, M., Dudoit, S., et al., 2004. Bioconductor: open software development for computational biology and bioinformatics. Genome Biology 5(10): R80.

[27] Bolstad, B.M., Irizarry, R.A., Astrand, M., Speed, T.P., 2003. A comparison of normalization methods for high density oligonucleotide array data based on variance and bias. Bioinformatics 19(2): 185-93.

[28] Irizarry, R.A., Bolstad, B.M., Collin, F., Cope, L.M., Hobbs, B., Speed, T.P., 2003. Summaries of Affymetrix GeneChip probe level data. Nucleic Acids Research 31(4): e15.

[29] Dai, M., Wang, P., Boyd, A.D., Kostov, G., Athey, B., Jones, E.G., et al., 2005. Evolving gene/transcript definitions significantly alter the interpretation of GeneChip data. Nucleic Acids Research 33(20): 1-9.

[30] Sartor, M.A., Tomlinson, C.R., Wesselkamper, S.C., Sivaganesan, S., Leikauf, G.D., Medvedovic, M., 2006. Intensity-based hierarchical Bayes method improves testing for differentially expressed genes in microarray experiments. BMC Bioinformatics 7: $1-17$.

[31] Subramaniana, A., Tamayoa, P., Moothaa, V.K., Mukherjeed, S., Eberta, B.L., Gillettea, M.A., et al., 2005. Gene set enrichment analysis: A knowledge-based approach for interpreting genome-wide expression profiles. Pnas 102(43): 1554550.

[32] Ruijter, J.M., Ramakers, C., Hoogaars, W.M.H., Karlen, Y., Bakker, O., van den hoff, M.J.B., et al., 2009. Amplification efficiency: Linking baseline and bias in the analysis of quantitative PCR data. Nucleic Acids Research 37(6).

[33] Pfaffl, M.W., 2001. A new mathematical model for relative quantification in realtime RT-PCR. Nucleic Acids Res 29(9): 16-21.

[34] Henstridge, D.C., Bruce, C.R., Pang, C.P., Lancaster, G.I., Allen, T.L., Estevez, E., et al., 2012. Skeletal muscle-specific overproduction of constitutively activated cJun N-terminal kinase (JNK) induces insulin resistance in mice. Diabetologia 55(10): 2769-78.

[35] Röhl, M., Pasparakis, M., Baudler, S., Baumgartl, J., Gautam, D., Huth, M., et al., 2004. Conditional disruption of IкB kinase 2 fails to prevent obesity-induced insulin resistance. Journal of Clinical Investigation 113(3): 474-81.

[36] White, A.T., LaBarge, S. a., McCurdy, C.E., Schenk, S., 2015. Knockout of STAT3 in skeletal muscle does not prevent high-fat diet-induced insulin resistance. Molecular Metabolism 4(8): 569-75.

[37] Polkinghorne, E., Lau, Q., Cooney, G.J., Kraegen, E.W., Cleasby, M.E., 2008. Local

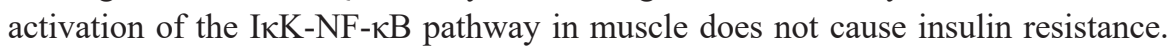
Am J Physiol Endocrinol Metab 294: 316-25.

[38] Tateya, S., Tamori, Y., Kawaguchi, T., Kanda, H., Kasuga, M., 2010. An increase in the circulating concentration of monocyte chemoattractant protein-1 elicits 
systemic insulin resistance irrespective of adipose tissue inflammation in mice. Endocrinology 151(3): 971-9.

[39] Ruggiero, P., Flati, S., Di Cioccio, V., Maurizi, G., Macchia, G., Facchin, A., et al., 2003. Glycosylation enhances functional stability of the chemotactic cytokine CCL2. European Cytokine Network 14(2): 91-6.

[40] Liu, Z.G., Haelens, A., Wuyts, A., Struyf, S., Pang, X.W., Proost, P., et al., 1996. Isolation of a lymphocyte chemotactic factor produced by the murine thymic epithelial cell line MTEC1: identification as a $30 \mathrm{kDa}$ glycosylated form of MCP-1. Eur Cytokine Netw 7: 381-388.

[41] Zhang, Y., Ernst, C.A., Rollins, B.J., 1996. MCP-1: Structure/Activity Analysis. Methods 10(1): 93-103.

[42] Yao, Y., Tsirka, S.E., 2010. The C terminus of Mouse Monocyte Chemoattractant Protein 1 (MCP1) mediates MCP1 dimerization while blocking its chemotactic potency. Journal of Biological Chemistry 285(41): 31509-16.

[43] Bastard, J.-P., Maachi, M., Van Nhieu, J.T., Jardel, C., Bruckert, E., Grimaldi, A., et al., 2002. Adipose tissue IL-6 content correlates with resistance to insulin activation of glucose uptake both in vivo and in vitro. Journal of Clinical Endocrinology and Metabolism 87(5): 2084-9.

[44] Kim, J.H., Jae, E.K., Liu, H.Y., Cao, W., Chen, J., 2008. Regulation of interleukin6-induced hepatic insulin resistance by mammalian target of rapamycin through the STAT3-SOCS3 pathway. Journal of Biological Chemistry 283(2): 708-15.

[45] Senn, J.J., Klover, P.J., Nowak, I.A., Mooney, R.A., 2002. Interleukin-6 induces cellular insulin resistance in hepatocytes. Diabetes 51(12): 3391-9. 


\section{Supplemental Material}

liver

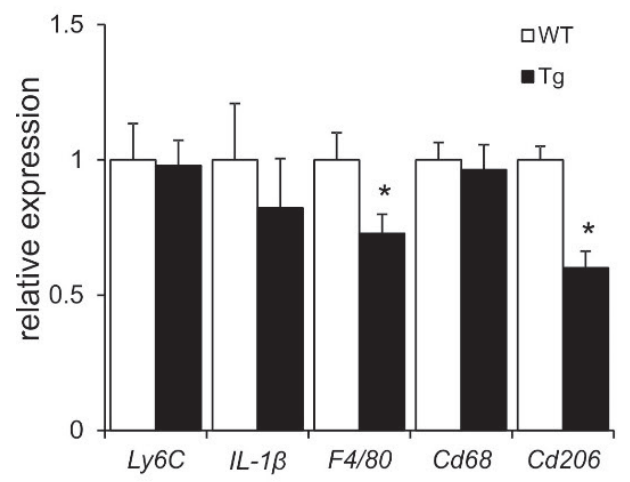

white adipose tissue

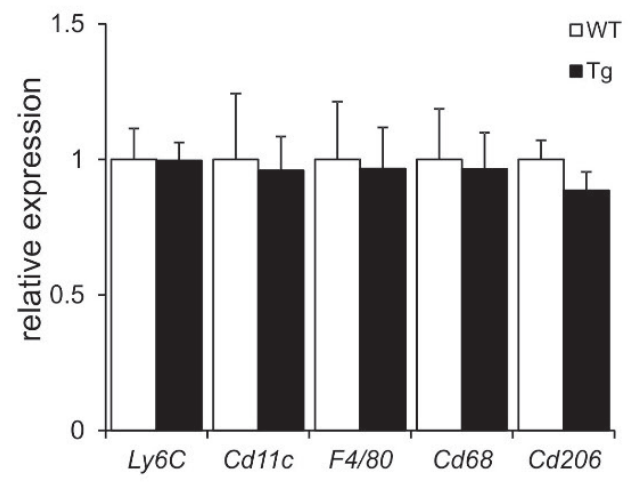

Supplemental Figure 1: Relative gene expression of selected genes in liver and white adipose tissue measured by RT-PCR; $n=12$ [WT], 12 [Mcp-1-Tg]. Data are means \pm $\mathrm{SEM} ; *=p<0.05$ 


\section{Supplemental Table 1: Primer sequences of genes}

\begin{tabular}{|c|c|c|}
\hline Genes & Forward primer & Reverse primer \\
\hline m36B4 & AGCGCGTCCTGGCATTGTCTGTGG & GGGCAGCAGTGGTGGCAGCAGC \\
\hline $\mathrm{mCd} 68$ & CCAATTCAGGGTGGAAGAAA & CTCGGGCTCTGATGTAGGTC \\
\hline $\mathrm{mF} 4 / 80$ & CTTTGGCTATGGGCTTCCAGTC & GCAAGGAGGACAGAGTTTATCGTG \\
\hline mIl-1b & CAGGCAGGCAGTATCACTCA & AGGTGCTCATGTCCTCATCC \\
\hline mIl-6 & CTTCCATCCAGTTGCCTTCTTG & AATTAAGCCTCCGACTTGTGAAG \\
\hline mLy6c & GCAGTGCTACGAGTGCTATGG & ACTGACGGGTCTTTAGTTTCCTT \\
\hline $\mathrm{mCd} 206$ & CTCTGTTCAGCTATTGGACGC & CGGAATTTCTGGGATTCAGCTTC \\
\hline mSrebplc & GGAGCCATGGATTGCACATT & CCTGTCTCACCCCCAGCATA \\
\hline $\mathrm{mGk}$ & AGGAGGCCAGTGTAAAGATGT & CTCCCAGGTCTAAGGAGAGAAA \\
\hline mAcc & GCCATTGGTATTGGGGCTTACC & CCCGACCAAGGACTTTGTTG \\
\hline mPepck & CAGGATCGAAAAGCAAGACAGT & AAGTCCTCTTCCGACATCCAG \\
\hline
\end{tabular}




\section{Supplemental Table 2: Genes significantly altered between gastrocnemius of WT and $M c p-1-T g$ mice $(\mathbf{q}<0.05)$}

\begin{tabular}{|c|c|c|c|c|}
\hline Entrez ID & $\begin{array}{l}\text { Gene } \\
\text { name }\end{array}$ & $\begin{array}{l}\text { Fold } \\
\text { change }\end{array}$ & q value & Description \\
\hline 20296 & $\mathrm{Ccl} 2$ & 99.11 & $0.00 \mathrm{E}+00$ & chemokine (C-C motif) ligand 2 \\
\hline 12772 & Ccr2 & 6.90 & $2.09 \mathrm{E}-10$ & chemokine (C-C motif) receptor 2 \\
\hline 17110 & Lyz1 & 5.76 & $5.22 \mathrm{E}-10$ & lysozyme 1 \\
\hline 57262 & Retnla & 5.73 & $6.48 \mathrm{E}-08$ & resistin like alpha \\
\hline 14961 & $\mathrm{H} 2-\mathrm{Ab} 1$ & 5.34 & $2.33 \mathrm{E}-10$ & histocompatibility 2 , class II antigen A, beta 1 \\
\hline 13587 & Ear2 & 5.08 & $2.10 \mathrm{E}-05$ & eosinophil-associated, ribonuclease A family, member 2 \\
\hline 14960 & $\mathrm{H} 2-\mathrm{Aa}$ & 4.88 & $2.55 \mathrm{E}-09$ & histocompatibility 2 , class II antigen A, alpha \\
\hline 16149 & $\mathrm{Cd} 74$ & 4.79 & $3.58 \mathrm{E}-10$ & CD74 antigen (invariant polypeptide ofMHC, class II antigen- \\
\hline \multicolumn{5}{|r|}{ - } \\
\hline 14969 & $\mathrm{H} 2-\mathrm{Eb} 1$ & 4.74 & 5.85E-09 & histocompatibility 2 , class II antigen E beta \\
\hline 23833 & Cd52 & 4.31 & $8.65 \mathrm{E}-09$ & CD52 antigen \\
\hline 68169 & Ndnf & 4.17 & 1.17E-09 & neuron-derived neurotrophic factor \\
\hline 80891 & Fcrls & 3.87 & $1.23 \mathrm{E}-07$ & Fc receptor-like $S$, scavenger receptor \\
\hline 17079 & $\mathrm{Cd} 180$ & 3.81 & $6.87 \mathrm{E}-10$ & CD180 antigen \\
\hline 98365 & Slamf9 & 3.80 & $5.23 \mathrm{E}-09$ & SLAM family member 9 \\
\hline 17476 & Mpeg1 & 3.58 & $1.22 \mathrm{E}-07$ & macrophage expressed gene 1 \\
\hline 14999 & $\mathrm{H} 2-\mathrm{DMb} 1$ & 3.43 & 2.14E-03 & histocompatibility 2 , class II, locus Mb1 \\
\hline 14998 & H2-DMa & 3.36 & $1.64 \mathrm{E}-08$ & histocompatibility 2, class II, locus DMa \\
\hline 17084 & Ly86 & 3.32 & $1.80 \mathrm{E}-06$ & lymphocyte antigen 86 \\
\hline 12982 & Csf2ra & 3.24 & $6.48 \mathrm{E}-08$ & colony stimulating factor 2 receptor, alpha, low-affinity (granulocyte- \\
\hline \multicolumn{5}{|r|}{$\mathrm{x}^{2}$} \\
\hline 16792 & Laptm5 & 3.22 & $2.55 \mathrm{E}-09$ & lysosomal-associated protein transmembrane 5 \\
\hline 11658 & Alcam & 3.21 & 4.03E-04 & activated leukocyte cell adhesion molecule \\
\hline 241633 & Atp8b4 & 3.15 & $1.98 \mathrm{E}-07$ & ATPase, class I, type 8B, member 4 \\
\hline 19264 & Ptprc & 3.12 & 4.85E-08 & protein tyrosine phosphatase, receptor type, $\mathrm{C}$ \\
\hline 17105 & Lyz2 & 3.07 & $1.82 \mathrm{E}-06$ & lysozyme 2 \\
\hline 231805 & Pilra & 3.02 & $1.22 \mathrm{E}-07$ & paired immunoglobin-like type 2 receptor alpha \\
\hline 12523 & $\mathrm{Cd} 84$ & 2.99 & $1.82 \mathrm{E}-06$ & CD84 antigen \\
\hline 213391 & Rassf4 & 2.98 & 4.85E-08 & Ras association (RalGDS/AF-6) domain family member 4 \\
\hline 13723 & $\mathrm{Emb}$ & 2.98 & 5.93E-06 & embigin \\
\hline 12508 & $\mathrm{Cd} 53$ & 2.91 & $2.77 \mathrm{E}-07$ & CD53 antigen \\
\hline 227929 & Cytip & 2.88 & $1.52 \mathrm{E}-06$ & cytohesin 1 interacting protein \\
\hline 104759 & Pld4 & 2.88 & 9.84E-08 & phospholipase D family, member 4 \\
\hline 216864 & Mg12 & 2.85 & $3.28 \mathrm{E}-08$ & macrophage galactose $\mathrm{N}$-acetyl-galactosamine specific lectin 2 \\
\hline 225825 & $\mathrm{Cd} 226$ & 2.84 & $1.29 \mathrm{E}-06$ & CD226 antigen \\
\hline 213002 & Ifitm6 & 2.81 & 8.04E-06 & interferon induced transmembrane protein 6 \\
\hline 17381 & Mmp12 & 2.75 & $2.52 \mathrm{E}-06$ & matrix metallopeptidase 12 \\
\hline 20345 & Selplg & 2.75 & $2.37 \mathrm{E}-07$ & selectin, platelet (p-selectin) ligand \\
\hline 217305 & Cd3001d & 2.73 & $1.92 \mathrm{E}-05$ & CD300 molecule-like family member d \\
\hline 72318 & Cyth4 & 2.73 & $5.85 \mathrm{E}-09$ & cytohesin 4 \\
\hline 16414 & Itgb2 & 2.72 & 4.85E-08 & integrin beta 2 \\
\hline 18726 & Lilra6 & 2.70 & $6.53 \mathrm{E}-04$ & leukocyte immunoglobulin-like receptor, subfamily A (with TM \\
\hline \multicolumn{5}{|c|}{ domain), member 6} \\
\hline 94176 & Dock2 & 2.70 & $1.25 \mathrm{E}-07$ & dedicator of cyto-kinesis 2 \\
\hline 216984 & Evi2b & 2.68 & $1.52 \mathrm{E}-06$ & ecotropic viral integration site $2 b$ \\
\hline 16409 & Itgam & 2.66 & $6.60 \mathrm{E}-07$ & integrin alpha $\mathrm{M}$ \\
\hline \multicolumn{2}{|c|}{100038909} & Gm14548 & 2.64 & $1.01 \mathrm{E}-03$ predicted gene 14548 \\
\hline 20375 & Spi1 & 2.64 & $8.65 \mathrm{E}-09$ & spleen focus forming virus (SFFV) proviral integration oncogene \\
\hline 12721 & Corola & 2.59 & 2.37E-07 & coronin, actin binding protein $1 \mathrm{~A}$ \\
\hline 414084 & Tnip3 & 2.58 & $1.42 \mathrm{E}-07$ & TNFAIP3 interacting protein 3 \\
\hline 14728 & Lilrb4 & 2.58 & $1.29 \mathrm{E}-06$ & leukocyte immunoglobulin-like receptor, subfamily B, member 4 \\
\hline 19260 & Ptpn22 & 2.55 & $1.25 \mathrm{E}-07$ & protein tyrosine phosphatase, non-receptor type 22 (lymphoid) \\
\hline 14727 & Gp49a & 2.55 & $5.40 \mathrm{E}-05$ & glycoprotein $49 \mathrm{~A}$ \\
\hline
\end{tabular}


Supplemental Table 2 continued

\begin{tabular}{|c|c|c|}
\hline 319446 & Dpep2 & 2.54 \\
\hline 627984 & Nlrp1c-ps & 2.54 \\
\hline 20308 & $\mathrm{Ccl} 9$ & 2.52 \\
\hline 13058 & Cybb & 2.50 \\
\hline 394432 & Ugt1a7c & 2.49 \\
\hline 11690 & Alox5ap & 2.48 \\
\hline 20305 & $\mathrm{Ccl} 6$ & 2.48 \\
\hline 13040 & Ctss & 2.46 \\
\hline 18636 & Cfp & 2.46 \\
\hline 74039 & Nfam1 & 2.45 \\
\hline 11501 & Adam8 & 2.43 \\
\hline 69810 & Clec4b1 & 2.43 \\
\hline 232413 & Clec12a & 2.43 \\
\hline 66815 & Ccdc109b & 2.40 \\
\hline 230787 & Themis2 & 2.37 \\
\hline 18830 & Pltp & 2.37 \\
\hline 14127 & Fcer1g & 2.35 \\
\hline 16822 & Lcp2 & 2.33 \\
\hline 56644 & Clec7a & 2.31 \\
\hline 13449 & Dok2 & 2.28 \\
\hline 21391 & Tbxas 1 & 2.27 \\
\hline 107684 & Coro2a & 2.24 \\
\hline 12265 & Ciita & 2.24 \\
\hline 15000 & $\mathrm{H} 2-\mathrm{DMb} 2$ & 2.23 \\
\hline 19204 & Ptafr & 2.21 \\
\hline 18826 & Lcp1 & 2.20 \\
\hline 19277 & Ptpro & 2.18 \\
\hline 72042 & Cotl1 & 2.17 \\
\hline \multicolumn{2}{|c|}{100038363} & RIKEN \\
\hline 12514 & Cd68 & 2.15 \\
\hline 75345 & Slamf7 & 2.11 \\
\hline 105855 & Nckap11 & 2.11 \\
\hline 18301 & Fxyd5 & 2.10 \\
\hline 212937 & Tifab & 2.10 \\
\hline \multicolumn{3}{|c|}{ member B } \\
\hline 12481 & $\mathrm{Cd} 2$ & 2.09 \\
\hline 18796 & Plcb2 & 2.08 \\
\hline 16854 & Lgals3 & 2.06 \\
\hline 12260 & $\mathrm{Clqb}$ & 2.05 \\
\hline 22177 & Tyrobp & 2.05 \\
\hline 73149 & Clec4a3 & 2.04 \\
\hline 12493 & $\mathrm{Cd} 37$ & 2.02 \\
\hline 19354 & $\operatorname{Rac} 2$ & 2.01 \\
\hline 503550 & Klri1 & 1.95 \\
\hline 56792 & Stap1 & 1.95 \\
\hline 20491 & Sla & 1.94 \\
\hline 26904 & Sh2d1b1 & 1.94 \\
\hline 226652 & Arhgap30 & 1.92 \\
\hline 76088 & Dock8 & 1.91 \\
\hline 98496 & Pid1 & 1.89 \\
\hline 12506 & $\mathrm{Cd} 48$ & 1.89 \\
\hline 54445 & Unc93b1 & 1.89 \\
\hline 668218 & $\operatorname{Bin} 2$ & 1.89 \\
\hline 22778 & Ikzf1 & 1.89 \\
\hline 20351 & Sema4a & 1.89 \\
\hline 17969 & Ncf1 & 1.88 \\
\hline 58861 & Cysltr1 & 1.87 \\
\hline
\end{tabular}

1.70E-06 dipeptidase 2

1.13E-05 NLR family, pyrin domain containing 1C, pseudogene

8.11E-06 chemokine (C-C motif) ligand 9

$1.44 \mathrm{E}-06$ cytochrome $\mathrm{b}-245$, beta polypeptide

2.60E-04 UDP glucuronosyltransferase 1 family, polypeptide A7C

2.04E-06 arachidonate 5-lipoxygenase activating protein

7.78E-05 chemokine (C-C motif) ligand 6

5.68E-06 cathepsin S

$1.80 \mathrm{E}-06$ complement factor properdin

5.98E-07 Nfat activating molecule with ITAM motif 1

6.23E-09 a disintegrin and metallopeptidase domain 8

3.50E-03 C-type lectin domain family 4 , member b1

1.00E-05 C-type lectin domain family 12 , member a

8.55E-07 coiled-coil domain containing 109B

$1.52 \mathrm{E}-06$ thymocyte selection associated family member 2

$1.15 \mathrm{E}-08$ phospholipid transfer protein

6.48E-08 Fc receptor, IgE, high affinity I, gamma polypeptide

4.33E-07 lymphocyte cytosolic protein 2

3.86E-05 C-type lectin domain family 7 , member a

$1.71 \mathrm{E}-06$ docking protein 2

3.45E-07 thromboxane A synthase 1, platelet

$2.12 \mathrm{E}-05$ coronin, actin binding protein $2 \mathrm{~A}$

$1.37 \mathrm{E}-06$ class II transactivator

6.28E-04 histocompatibility 2, class II, locus Mb2

$3.73 \mathrm{E}-06$ platelet-activating factor receptor

4.02E-05 lymphocyte cytosolic protein 1

$1.01 \mathrm{E}-02$ protein tyrosine phosphatase, receptor type, $\mathrm{O}$

2.71E-08 coactosin-like 1 (Dictyostelium)

2.16 1.44E-03 RIKEN cDNA F630028010 gene

4.84E-05 CD68 antigen

$1.03 \mathrm{E}-05$ SLAM family member 7

2.18E-07 NCK associated protein 1 like

1.37E-05 FXYD domain-containing ion transport regulator 5

6.98E-06 TRAF-interacting protein with forkhead-associated domain, family

1.82E-06 CD2 antigen

7.80E-05 phospholipase $\mathrm{C}$, beta 2

7.78E-05 lectin, galactose binding, soluble 3

8.60E-06 complement component 1 , q subcomponent, beta polypeptide

6.02E-04 TYRO protein tyrosine kinase binding protein

$1.03 \mathrm{E}-04$ C-type lectin domain family 4 , member a3

1.00E-05 CD37 antigen

1.54E-03 RAS-related C3 botulinum substrate 2

1.66E-04 killer cell lectin-like receptor family I member 1

$1.00 \mathrm{E}-05$ signal transducing adaptor family member 1

$2.10 \mathrm{E}-05$ src-like adaptor

4.48E-03 SH2 domain protein 1B1

2.69E-05 Rho GTPase activating protein 30

$1.29 \mathrm{E}-06$ dedicator of cytokinesis 8

$1.15 \mathrm{E}-06$ phosphotyrosine interaction domain containing 1

2.82E-05 CD48 antigen

3.12E-05 unc-93 homolog B1 (C. elegans)

$1.07 \mathrm{E}-03$ bridging integrator 2

2.30E-05 IKAROS family zinc finger 1

2.83E-05 Semaphorin 4A

8.81E-05 neutrophil cytosolic factor 1

7.30E-04 cysteinyl leukotriene receptor 1 
Supplemental Table 2 continued

\begin{tabular}{|c|c|c|c|c|}
\hline 19267 & Ptpre & 1.87 & $5.72 \mathrm{E}-05$ & protein tyrosine phosphatase, receptor type, E \\
\hline 52614 & Emr4 & 1.87 & $2.71 \mathrm{E}-03$ & EGF-like module containing, mucin-like, hormone receptor-like \\
\hline \multicolumn{5}{|r|}{ 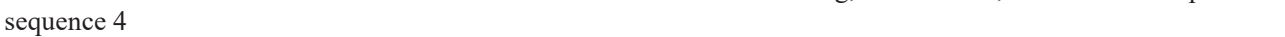 } \\
\hline 78416 & Rnase6 & 1.87 & $2.30 \mathrm{E}-05$ & ribonuclease, RNase A family, 6 \\
\hline 17345 & Mki67 & 1.86 & $1.51 \mathrm{E}-03$ & antigen identified by monoclonal antibody Ki 67 \\
\hline 26411 & Map4k1 & 1.86 & $1.37 \mathrm{E}-05$ & mitogen-activated protein kinase kinase kinase kinase 1 \\
\hline 56177 & Olfm1 & 1.85 & $1.78 \mathrm{E}-02$ & olfactomedin 1 \\
\hline 14421 & B4galnt1 & 1.84 & $1.25 \mathrm{E}-03$ & beta-1,4-N-acetyl-galactosaminyl transferase 1 \\
\hline 73723 & Sh3bgrl3 & 1.84 & $2.83 \mathrm{E}-05$ & SH3 domain binding glutamic acid-rich protein-like 3 \\
\hline 64381 & Ms4a8a & 1.83 & $1.50 \mathrm{E}-03$ & membrane-spanning 4-domains, subfamily A, member 8A \\
\hline 239217 & Kctd12 & 1.82 & $5.93 \mathrm{E}-06$ & potassium channel tetramerisation domain containing 12 \\
\hline 320139 & Ptpn7 & 1.81 & 1.39E-03 & protein tyrosine phosphatase, non-receptor type 7 \\
\hline 14130 & Fcgr $2 b$ & 1.80 & $1.61 \mathrm{E}-04$ & Fc receptor, IgG, low affinity IIb \\
\hline 16364 & Irf4 & 1.79 & $2.80 \mathrm{E}-02$ & interferon regulatory factor 4 \\
\hline 18718 & Pip4k2a & 1.79 & $6.47 \mathrm{E}-05$ & phosphatidylinositol-5-phosphate 4-kinase, type II, alpha \\
\hline 80719 & Igsf6 & 1.79 & 4.62E-04 & immunoglobulin superfamily, member 6 \\
\hline 17916 & Myo1f & 1.79 & 7.78E-05 & myosin IF \\
\hline 545030 & Wdfy4 & 1.79 & $2.10 \mathrm{E}-04$ & WD repeat and FYVE domain containing 4 \\
\hline 14131 & Fcgr3 & 1.79 & 3.37E-03 & Fc receptor, IgG, low affinity III \\
\hline 81897 & Tlr9 & 1.78 & $6.31 \mathrm{E}-05$ & toll-like receptor 9 \\
\hline 65963 & \multicolumn{2}{|c|}{ Tmem $176 b$} & 1.78 & $2.35 \mathrm{E}-05$ transmembrane protein $176 \mathrm{~B}$ \\
\hline 14017 & Evi2a & 1.77 & $2.55 \mathrm{E}-04$ & ecotropic viral integration site $2 \mathrm{a}$ \\
\hline 11810 & Apobec 1 & 1.77 & $1.37 \mathrm{E}-05$ & apolipoprotein B mRNA editing enzyme, catalytic polypeptide 1 \\
\hline 246177 & Myolg & 1.77 & $1.16 \mathrm{E}-04$ & myosin IG \\
\hline 668139 & Gm8995 & 1.77 & $1.38 \mathrm{E}-04$ & predicted gene 8995 \\
\hline 20963 & Syk & 1.77 & $8.16 \mathrm{E}-04$ & spleen tyrosine kinase \\
\hline 71653 & RIKEN & 1.76 & $1.08 \mathrm{E}-04$ & RIKEN cDNA 4930506M07 gene \\
\hline 17082 & Il1rl1 & 1.76 & $5.03 \mathrm{E}-04$ & interleukin 1 receptor-like 1 \\
\hline 67111 & Naaa & 1.75 & $7.41 \mathrm{E}-07$ & $\mathrm{~N}$-acylethanolamine acid amidase \\
\hline 19261 & Sirpa & 1.75 & $6.28 \mathrm{E}-04$ & signal-regulatory protein alpha \\
\hline 20198 & $\mathrm{~S} 100 \mathrm{a} 4$ & 1.73 & $9.85 \mathrm{E}-03$ & S100 calcium binding protein A4 \\
\hline 170786 & $\mathrm{Cd} 209 \mathrm{a}$ & 1.72 & 7.73E-03 & CD209a antigen \\
\hline 210293 & Dock10 & 1.71 & 4.24E-04 & dedicator of cytokinesis 10 \\
\hline 236312 & Pyhin1 & 1.71 & $2.10 \mathrm{E}-04$ & pyrin and HIN domain family, member 1 \\
\hline 269799 & Clec4a1 & 1.71 & 4.91E-04 & C-type lectin domain family 4 , member a1 \\
\hline 64214 & Rgs18 & 1.71 & $1.00 \mathrm{E}-03$ & regulator of G-protein signaling 18 \\
\hline 12229 & Btk & 1.70 & $3.35 \mathrm{E}-03$ & Bruton agammaglobulinemia tyrosine kinase \\
\hline 18173 & Slc11a1 & 1.70 & $5.40 \mathrm{E}-05$ & solute carrier family 11 (proton-coupled divalent metal ion \\
\hline \multicolumn{5}{|c|}{ transporters), member 1} \\
\hline 26888 & Clec4a2 & 1.70 & $1.88 \mathrm{E}-03$ & C-type lectin domain family 4 , member a2 \\
\hline 69993 & Chn2 & 1.70 & 2.22E-02 & chimerin 2 \\
\hline 234779 & Plcg2 & 1.70 & $5.30 \mathrm{E}-04$ & phospholipase C, gamma 2 \\
\hline 21844 & Tiam1 & 1.70 & $2.60 \mathrm{E}-04$ & $\mathrm{~T}$ cell lymphoma invasion and metastasis 1 \\
\hline 57781 & $\mathrm{Cd} 200 \mathrm{r} 1$ & 1.70 & $1.54 \mathrm{E}-03$ & CD200 receptor 1 \\
\hline 13732 & Emp3 & 1.70 & $2.64 \mathrm{E}-05$ & epithelial membrane protein 3 \\
\hline 22228 & Ucp2 & 1.70 & $6.82 \mathrm{E}-04$ & uncoupling protein 2 (mitochondrial, proton carrier) \\
\hline 217304 & $\mathrm{Cd} 300 \mathrm{lb}$ & 1.70 & $2.35 \mathrm{E}-04$ & CD300 antigen like family member $\mathrm{B}$ \\
\hline 16541 & Napsa & 1.69 & $6.67 \mathrm{E}-03$ & napsin A aspartic peptidase \\
\hline 12505 & $\mathrm{Cd} 44$ & 1.69 & $1.52 \mathrm{E}-04$ & CD44 antigen \\
\hline 17444 & Grap2 & 1.69 & $6.22 \mathrm{E}-05$ & GRB2-related adaptor protein 2 \\
\hline 170744 & Tlr8 & 1.68 & $8.45 \mathrm{E}-03$ & toll-like receptor 8 \\
\hline 13733 & Emr1 & 1.68 & $8.12 \mathrm{E}-03$ & EGF-like module containing, mucin-like, hormone receptor-like \\
\hline \multicolumn{5}{|r|}{ ( } \\
\hline 10050446 & & RIKEN & 1.68 & 3.16E-04 RIKEN cDNA E230016K23 gene \\
\hline 72461 & Prcp & 1.67 & $6.22 \mathrm{E}-05$ & prolylcarboxypeptidase (angiotensinase C) \\
\hline 210029 & Metrnl & 1.67 & $1.59 \mathrm{E}-03$ & meteorin, glial cell differentiation regulator-like \\
\hline 14667 & $\mathrm{Gm} 2 \mathrm{a}$ & 1.66 & $6.05 \mathrm{E}-06$ & GM2 ganglioside activator protein \\
\hline
\end{tabular}


Supplemental Table 2 continued

\begin{tabular}{|c|c|c|c|c|}
\hline 14457 & Gas 7 & 1.66 & $6.22 \mathrm{E}-05$ & growth arrest specific 7 \\
\hline 14191 & Fgr & 1.66 & $2.38 \mathrm{E}-05$ & Gardner-Rasheed feline sarcoma viral (Fgr) oncogene homolog \\
\hline 83490 & Pik3ap1 & 1.66 & $1.96 \mathrm{E}-04$ & phosphoinositide-3-kinase adaptor protein 1 \\
\hline 16331 & Inpp5d & 1.66 & $1.34 \mathrm{E}-04$ & inositol polyphosphate-5-phosphatase D \\
\hline 108101 & Fermt 3 & 1.65 & 1.17E-03 & fermitin family homolog 3 (Drosophila) \\
\hline 16534 & Kcnn4 & 1.65 & $2.07 \mathrm{E}-04$ & potassium intermediate/small conductance Ca-activ. channel, subfam \\
\hline \multicolumn{5}{|c|}{$\mathrm{N}$, member 4} \\
\hline 66058 & \multicolumn{2}{|c|}{ Tmem176a } & 1.65 & 7.78E-05 transmembrane protein 176A \\
\hline 15170 & Ptpn6 & 1.65 & $2.56 \mathrm{E}-04$ & protein tyrosine phosphatase, non-receptor type 6 \\
\hline 22350 & Ezr & 1.64 & $4.28 \mathrm{E}-05$ & ezrin \\
\hline 224829 & Trerf1 & 1.63 & $5.29 \mathrm{E}-05$ & transcriptional regulating factor 1 \\
\hline 224109 & Nrros & 1.62 & 4.92E-03 & negative regulator of reactive oxygen species \\
\hline 74131 & Sash3 & 1.61 & $3.11 \mathrm{E}-03$ & SAM and SH3 domain containing 3 \\
\hline 19271 & Ptprj & 1.61 & $2.12 \mathrm{E}-05$ & protein tyrosine phosphatase, receptor type, $\mathrm{J}$ \\
\hline 12267 & C3ar1 & 1.60 & $1.34 \mathrm{E}-03$ & complement component 3 a receptor 1 \\
\hline 238875 & Gapt & 1.59 & $7.38 \mathrm{E}-03$ & Grb2-binding adaptor, transmembrane \\
\hline 13057 & Cyba & 1.59 & $7.10 \mathrm{E}-03$ & cytochrome b-245, alpha polypeptide \\
\hline 70719 & Hmhal & 1.59 & $4.60 \mathrm{E}-03$ & histocompatibility (minor) HA-1 \\
\hline 16154 & Il10ra & 1.58 & $4.03 \mathrm{E}-03$ & interleukin 10 receptor, alpha \\
\hline 75767 & Rab11fip1 & 1.58 & $2.14 \mathrm{E}-03$ & RAB11 family interacting protein 1 (class I) \\
\hline 56470 & Rgs19 & 1.58 & $1.58 \mathrm{E}-04$ & regulator of G-protein signaling 19 \\
\hline 67742 & Samsn 1 & 1.57 & $3.52 \mathrm{E}-03$ & SAM domain, SH3 domain and nuclear localization signals, 1 \\
\hline 320181 & Fndc7 & 1.56 & $1.88 \mathrm{E}-05$ & fibronectin type III domain containing 7 \\
\hline 382062 & AB124611 & 1.56 & $2.51 \mathrm{E}-02$ & cDNA sequence AB124611 \\
\hline 170741 & Pilrb1 & 1.56 & $5.22 \mathrm{E}-03$ & paired immunoglobin-like type 2 receptor beta 1 \\
\hline 72828 & Ubash $3 b$ & 1.55 & $6.61 \mathrm{E}-03$ & ubiquitin associated and $\mathrm{SH} 3$ domain containing, $\mathrm{B}$ \\
\hline 73690 & Glipr1 & 1.55 & $2.50 \mathrm{E}-03$ & GLI pathogenesis-related 1 (glioma) \\
\hline 12978 & Csf1r & 1.54 & $1.80 \mathrm{E}-03$ & colony stimulating factor 1 receptor \\
\hline 239081 & Tlr11 & 1.54 & $7.38 \mathrm{E}-03$ & toll-like receptor 11 \\
\hline 14268 & Fn1 & 1.54 & $1.15 \mathrm{E}-03$ & fibronectin 1 \\
\hline 227541 & Camk1d & 1.54 & $8.16 \mathrm{E}-04$ & calcium/calmodulin-dependent protein kinase ID \\
\hline 74127 & Krt80 & 1.54 & $1.24 \mathrm{E}-02$ & keratin 80 \\
\hline 76117 & Arhgap 15 & 1.54 & $1.38 \mathrm{E}-04$ & Rho GTPase activating protein 15 \\
\hline 72925 & March1 & 1.53 & $8.45 \mathrm{E}-03$ & membrane-associated ring finger $(\mathrm{C} 3 \mathrm{HC} 4) 1$ \\
\hline 107373 & Fam111a & 1.53 & $1.65 \mathrm{E}-02$ & family with sequence similarity 111, member A \\
\hline 70536 & Qpct & 1.53 & $5.03 \mathrm{E}-04$ & glutaminyl-peptide cyclotransferase (glutaminyl cyclase) \\
\hline 211228 & Lrrc25 & 1.53 & $7.46 \mathrm{E}-04$ & leucine rich repeat containing 25 \\
\hline 64095 & Gpr35 & 1.53 & $1.77 \mathrm{E}-04$ & G protein-coupled receptor 35 \\
\hline 27056 & Irf5 & 1.53 & $5.66 \mathrm{E}-03$ & interferon regulatory factor 5 \\
\hline 225471 & Ticam2 & 1.53 & $1.23 \mathrm{E}-02$ & toll-like receptor adaptor molecule 2 \\
\hline 22324 & Vav1 & 1.53 & $1.56 \mathrm{E}-02$ & vav 1 oncogene \\
\hline 64380 & $\mathrm{Ms} 4 \mathrm{a} 4 \mathrm{c}$ & 1.52 & $1.47 \mathrm{E}-02$ & membrane-spanning 4-domains, subfamily A, member 4C \\
\hline 69769 & Tnfaip812 & 1.52 & $2.24 \mathrm{E}-04$ & tumor necrosis factor, alpha-induced protein 8 -like 2 \\
\hline 16970 & Lrmp & 1.52 & $6.02 \mathrm{E}-04$ & lymphoid-restricted membrane protein \\
\hline 16985 & Lsp1 & 1.52 & $1.59 \mathrm{E}-03$ & lymphocyte specific 1 \\
\hline 637515 & Nlrp1b & 1.52 & $1.69 \mathrm{E}-03$ & NLR family, pyrin domain containing 1B \\
\hline 320664 & Cass4 & 1.51 & $1.05 \mathrm{E}-02$ & Cas scaffolding protein family member 4 \\
\hline 19229 & Ptk2b & 1.51 & $2.02 \mathrm{E}-02$ & PTK2 protein tyrosine kinase 2 beta \\
\hline 64008 & Aqp9 & 1.51 & $3.96 \mathrm{E}-04$ & aquaporin 9 \\
\hline 27405 & Abcg3 & 1.50 & $5.03 \mathrm{E}-03$ & ATP-binding cassette, sub-family G (WHITE), member 3 \\
\hline 21810 & Tgfbi & 1.50 & $1.16 \mathrm{E}-04$ & transforming growth factor, beta induced \\
\hline 227659 & Slc2a6 & 1.50 & $5.92 \mathrm{E}-03$ & solute carrier family 2 (facilitated glucose transporter), member 6 \\
\hline 27984 & Efhd2 & 1.50 & $4.66 \mathrm{E}-04$ & EF hand domain containing 2 \\
\hline 14255 & Flt3 & 1.49 & $1.45 \mathrm{E}-02$ & FMS-like tyrosine kinase 3 \\
\hline 20970 & Sdc3 & 1.49 & $2.92 \mathrm{E}-03$ & syndecan 3 \\
\hline 11857 & Arhgdib & 1.49 & 4.91E-04 & Rho, GDP dissociation inhibitor (GDI) beta \\
\hline 70785 & Dennd1c & 1.49 & $1.05 \mathrm{E}-02$ & DENN/MADD domain containing 1C \\
\hline
\end{tabular}


Supplemental Table 2 continued

\begin{tabular}{|c|c|c|c|c|}
\hline 107321 & Lpxn & 1.48 & $2.83 \mathrm{E}-02$ & leupaxin \\
\hline 67865 & Rgs 10 & 1.48 & $2.89 \mathrm{E}-02$ & regulator of G-protein signalling 10 \\
\hline 19735 & Rgs2 & 1.48 & $4.85 \mathrm{E}-02$ & regulator of G-protein signaling 2 \\
\hline 20556 & Slfn2 & 1.48 & 8.03E-04 & schlafen 2 \\
\hline 320207 & Pik3r5 & 1.48 & $1.54 \mathrm{E}-02$ & phosphoinositide-3-kinase, regulatory subunit 5, p101 \\
\hline 215653 & Rassf2 & 1.48 & 4.54E-02 & Ras association (RalGDS/AF-6) domain family member 2 \\
\hline 54135 & Lsr & 1.48 & $4.00 \mathrm{E}-03$ & lipolysis stimulated lipoprotein receptor \\
\hline 27007 & Klrk1 & 1.48 & $5.03 \mathrm{E}-03$ & killer cell lectin-like receptor subfamily K, member 1 \\
\hline $\begin{array}{l}54519 \\
\text { interacting }\end{array}$ & $\begin{array}{l}\text { Apbblip } \\
\text { protein }\end{array}$ & 1.47 & 1.91E-02 & amyloid beta (A4) precursor protein-binding, family $\mathrm{B}$, member 1 \\
\hline 75766 & Destamp & 1.47 & $2.66 \mathrm{E}-02$ & dentrocyte expressed seven transmembrane protein \\
\hline 12475 & $\mathrm{Cd} 14$ & 1.47 & $1.32 \mathrm{E}-02$ & CD14 antigen \\
\hline 56743 & Lat2 & 1.47 & $5.66 \mathrm{E}-03$ & linker for activation of $\mathrm{T}$ cells family, member 2 \\
\hline 17970 & Nef2 & 1.47 & $3.76 \mathrm{E}-02$ & neutrophil cytosolic factor 2 \\
\hline 74748 & Slamf8 & 1.47 & $1.13 \mathrm{E}-02$ & SLAM family member 8 \\
\hline 26382 & Fgd2 & 1.46 & $1.47 \mathrm{E}-02$ & FYVE, RhoGEF and $\mathrm{PH}$ domain containing 2 \\
\hline 17952 & Naip6 & 1.46 & $5.03 \mathrm{E}-03$ & NLR family, apoptosis inhibitory protein 6 \\
\hline 68870 & Ak8 & 1.46 & $6.61 \mathrm{E}-03$ & adenylate kinase 8 \\
\hline 233046 & Rasgrp4 & 1.45 & $4.58 \mathrm{E}-02$ & RAS guanyl releasing protein 4 \\
\hline 544963 & Iqgap2 & 1.45 & $1.44 \mathrm{E}-03$ & IQ motif containing GTPase activating protein 2 \\
\hline 15163 & Hcls1 & 1.45 & $9.46 \mathrm{E}-03$ & hematopoietic cell specific Lyn substrate 1 \\
\hline 71607 & Snx20 & 1.45 & $2.22 \mathrm{E}-02$ & sorting nexin 20 \\
\hline 16633 & Klra2 & 1.44 & $2.56 \mathrm{E}-04$ & killer cell lectin-like receptor, subfamily A, member 2 \\
\hline 16411 & Itgax & 1.44 & 8.03E-04 & integrin alpha $\mathrm{X}$ \\
\hline 102595 & Plekho2 & 1.44 & $9.51 \mathrm{E}-04$ & pleckstrin homology domain containing, family $\mathrm{O}$ member 2 \\
\hline 330177 & Taok3 & 1.43 & $1.23 \mathrm{E}-03$ & TAO kinase 3 \\
\hline 16194 & Il6ra & 1.43 & $1.29 \mathrm{E}-02$ & interleukin 6 receptor, alpha \\
\hline 101202 & Hepacam2 & 1.43 & $1.25 \mathrm{E}-02$ & HEPACAM family member 2 \\
\hline 21934 & Tnfrsf11a & 1.43 & $1.52 \mathrm{E}-02$ & tumor necrosis factor receptor superfamily, member $11 \mathrm{a}$ \\
\hline 11867 & Arpc1b & 1.43 & $7.38 \mathrm{E}-03$ & actin related protein $2 / 3$ complex, subunit $1 \mathrm{~B}$ \\
\hline 14269 & Fnbpl & 1.42 & $3.89 \mathrm{E}-02$ & formin binding protein 1 \\
\hline 56193 & Plek & 1.42 & $6.61 \mathrm{E}-03$ & pleckstrin \\
\hline 23880 & Fyb & 1.42 & $1.28 \mathrm{E}-03$ & FYN binding protein \\
\hline 21803 & Tgfb1 & 1.41 & $1.72 \mathrm{E}-02$ & transforming growth factor, beta 1 \\
\hline 17095 & Lyl1 & 1.41 & $2.29 \mathrm{E}-02$ & lymphoblastomic leukemia 1 \\
\hline 29875 & Iqgap1 & 1.41 & 4.71E-04 & IQ motif containing GTPase activating protein 1 \\
\hline 108686 & Ccdc88a & 1.41 & 4.74E-02 & coiled coil domain containing $88 \mathrm{~A}$ \\
\hline 57442 & Kcne3 & 1.41 & $4.70 \mathrm{E}-03$ & potassium voltage-gated channel, Isk-related subfamily, gene 3 \\
\hline $\begin{array}{l}114644 \\
\text { member } 3\end{array}$ & Slc13a3 & 1.41 & $1.27 \mathrm{E}-02$ & solute carrier family 13 (sodium-dependent dicarboxylate transporter), \\
\hline 242248 & Bank1 & 1.41 & $1.26 \mathrm{E}-02$ & B cell scaffold protein with ankyrin repeats 1 \\
\hline 380732 & Milr1 & 1.41 & $4.54 \mathrm{E}-02$ & mast cell immunoglobulin like receptor 1 \\
\hline 214854 & Neur13 & 1.41 & $1.16 \mathrm{E}-02$ & neuralized homolog 3 homolog (Drosophila) \\
\hline 19241 & Tmsb4x & 1.40 & $5.14 \mathrm{E}-04$ & thymosin, beta $4, \mathrm{X}$ chromosome \\
\hline 383619 & $\operatorname{Aim} 2$ & 1.40 & 4.13E-03 & absent in melanoma 2 \\
\hline 16535 & Kenq1 & 1.40 & 4.39E-02 & potassium voltage-gated channel, subfamily $\mathrm{Q}$, member 1 \\
\hline 100182 & Akna & 1.40 & $1.94 \mathrm{E}-03$ & AT-hook transcription factor \\
\hline 72054 & Cyp4f18 & 1.40 & $1.16 \mathrm{E}-02$ & cytochrome P450, family 4, subfamily f, polypeptide 18 \\
\hline 18106 & $\mathrm{Cd} 244$ & 1.39 & $4.31 \mathrm{E}-03$ & CD244 natural killer cell receptor $2 \mathrm{~B} 4$ \\
\hline 11513 & Adcy 7 & 1.39 & $6.06 \mathrm{E}-03$ & adenylate cyclase 7 \\
\hline 233571 & P2ry6 & 1.38 & $2.75 \mathrm{E}-02$ & pyrimidinergic receptor $\mathrm{P} 2 \mathrm{Y}, \mathrm{G}$-protein coupled, 6 \\
\hline 20868 & Stk10 & 1.38 & $2.63 \mathrm{E}-02$ & serine/threonine kinase 10 \\
\hline 54354 & Rassf5 & 1.38 & $3.11 \mathrm{E}-02$ & Ras association (RalGDS/AF-6) domain family member 5 \\
\hline 226421 & RIKEN & 1.38 & $5.08 \mathrm{E}-03$ & RIKEN cDNA 5430435G22 gene \\
\hline 12331 & Cap1 & 1.38 & $7.96 \mathrm{E}-04$ & CAP, adenylate cyclase-associated protein 1 (yeast) \\
\hline 11630 & Aim 1 & 1.37 & $2.29 \mathrm{E}-02$ & absent in melanoma 1 \\
\hline 233406 & Prc1 & 1.37 & $1.32 \mathrm{E}-02$ & protein regulator of cytokinesis 1 \\
\hline
\end{tabular}


Supplemental Table 2 continued

\begin{tabular}{|c|c|c|c|c|}
\hline 56356 & Gltp & 1.37 & $8.46 \mathrm{E}-04$ & glycolipid transfer protein \\
\hline 12193 & Zfp3612 & 1.37 & $1.38 \mathrm{E}-02$ & zinc finger protein $36, \mathrm{C} 3 \mathrm{H}$ type-like 2 \\
\hline 22376 & Was & 1.37 & $1.00 \mathrm{E}-03$ & Wiskott-Aldrich syndrome homolog (human) \\
\hline 23790 & Corolc & 1.37 & $2.74 \mathrm{E}-04$ & coronin, actin binding protein $1 \mathrm{C}$ \\
\hline 57257 & Vav3 & 1.36 & 4.48E-03 & vav 3 oncogene \\
\hline 74735 & Trim14 & 1.36 & $1.41 \mathrm{E}-02$ & tripartite motif-containing 14 \\
\hline 13860 & Eps8 & 1.36 & $5.27 \mathrm{E}-03$ & epidermal growth factor receptor pathway substrate 8 \\
\hline 215632 & Psd4 & 1.36 & $5.09 \mathrm{E}-02$ & pleckstrin and Sec7 domain containing 4 \\
\hline 226101 & Myof & 1.35 & $2.20 \mathrm{E}-02$ & myoferlin \\
\hline 12798 & Cnn2 & 1.34 & $6.53 \mathrm{E}-03$ & calponin 2 \\
\hline 66494 & Prelid1 & 1.34 & $3.43 \mathrm{E}-02$ & PRELI domain containing 1 \\
\hline 107766 & Haаo & 1.34 & $1.27 \mathrm{E}-02$ & 3-hydroxyanthranilate 3,4-dioxygenase \\
\hline 223753 & Cerk & 1.34 & 4.07E-02 & ceramide kinase \\
\hline 17972 & Ncf4 & 1.34 & 4.67E-02 & neutrophil cytosolic factor 4 \\
\hline 56318 & Acpp & 1.34 & 4.78E-02 & acid phosphatase, prostate \\
\hline 217203 & \multicolumn{2}{|c|}{ Tmem106a } & 1.33 & $2.75 \mathrm{E}-02$ transmembrane protein $106 \mathrm{~A}$ \\
\hline 11461 & Actb & 1.32 & 7.13E-04 & actin, beta \\
\hline 328660 & Bex6 & 1.31 & 4.49E-02 & brain expressed gene 6 \\
\hline 12442 & Ccnb2 & 1.31 & 3.59E-02 & cyclin B2 \\
\hline 12259 & C1qa & 1.31 & 4.93E-02 & complement component $1, \mathrm{q}$ subcomponent, alpha polypeptide \\
\hline 241197 & Serpinb10 & 1.31 & $3.88 \mathrm{E}-02$ & serine (or cysteine) peptidase inhibitor, clade B (ovalbumin), member \\
\hline 10 & & & & \\
\hline 71279 & Slc29a3 & 1.29 & 8.22E-03 & solute carrier family 29 (nucleoside transporters), member 3 \\
\hline 16889 & Lipa & 1.28 & 3.89E-02 & lysosomal acid lipase A \\
\hline 13710 & Elf3 & 1.28 & $9.88 \mathrm{E}-03$ & E74-like factor 3 \\
\hline 110835 & Chrna5 & 1.28 & $3.10 \mathrm{E}-02$ & cholinergic receptor, nicotinic, alpha polypeptide 5 \\
\hline 16643 & Klrd1 & 1.27 & $2.97 \mathrm{E}-02$ & killer cell lectin-like receptor, subfamily D, member 1 \\
\hline 330460 & \multicolumn{2}{|c|}{ Tmem $150 \mathrm{~b}$} & 1.27 & 4.44E-02 transmembrane protein $150 \mathrm{~B}$ \\
\hline 223666 & \multicolumn{2}{|c|}{ Arhgap39 1.27} & $4.00 \mathrm{E}-02$ & Rho GTPase activating protein 39 \\
\hline 12489 & $\mathrm{Cd} 33$ & 1.27 & $2.80 \mathrm{E}-02$ & CD33 antigen \\
\hline 244209 & Cyp2r1 & 1.26 & $3.06 \mathrm{E}-02$ & cytochrome P450, family 2 , subfamily r, polypeptide 1 \\
\hline 238377 & Gpr68 & 1.25 & $3.64 \mathrm{E}-02$ & G protein-coupled receptor 68 \\
\hline 12306 & Anxa2 & 1.24 & $2.38 \mathrm{E}-02$ & annexin $\mathrm{A} 2$ \\
\hline 18438 & P2rx4 & 1.23 & $2.75 \mathrm{E}-02$ & purinergic receptor $\mathrm{P} 2 \mathrm{X}$, ligand-gated ion channel 4 \\
\hline 68598 & Dnajc8 & -1.23 & 2.29E-02 & DnaJ (Hsp40) homolog, subfamily C, member 8 \\
\hline 381816 & RIKEN & -1.23 & $3.48 \mathrm{E}-02$ & RIKEN cDNA 4922502D21 gene \\
\hline 11529 & Adh7 & -1.24 & $4.66 \mathrm{E}-02$ & alcohol dehydrogenase 7 (class IV), mu or sigma polypeptide \\
\hline 70385 & Spdl1 & -1.25 & $2.12 \mathrm{E}-02$ & spindle apparatus coiled-coil protein 1 \\
\hline 213006 & Mfsd4 & -1.27 & $3.44 \mathrm{E}-02$ & major facilitator superfamily domain containing 4 \\
\hline 244329 & Mcph1 & -1.27 & 4.33E-02 & microcephaly, primary autosomal recessive 1 \\
\hline 105083 & Pelo & -1.28 & 3.79E-02 & pelota homolog (Drosophila) \\
\hline 67390 & Rnmtl1 & -1.29 & 2.73E-02 & RNA methyltransferase like 1 \\
\hline 12971 & Crym & -1.31 & $2.13 \mathrm{E}-02$ & crystallin, mu \\
\hline 52653 & Nudcd2 & -1.33 & 4.82E-02 & NudC domain containing 2 \\
\hline 74096 & Hven 1 & -1.34 & $2.86 \mathrm{E}-02$ & hydrogen voltage-gated channel 1 \\
\hline 242519 & Ifna12 & -1.37 & $1.97 \mathrm{E}-02$ & interferon alpha 12 \\
\hline 17300 & Foxc1 & -1.38 & $2.29 \mathrm{E}-02$ & forkhead box $\mathrm{C} 1$ \\
\hline 18782 & Pla2g2d & -1.53 & $2.52 \mathrm{E}-02$ & phospholipase A2, group IID \\
\hline 114332 & Lyve1 & -1.68 & $1.01 \mathrm{E}-02$ & lymphatic vessel endothelial hyaluronan receptor 1 \\
\hline 276891 & Timd4 & -1.74 & $7.08 \mathrm{E}-04$ & T cell immunoglobulin and mucin domain containing 4 \\
\hline 14276 & Folr2 & -1.82 & $1.65 \mathrm{E}-02$ & folate receptor 2 (fetal) \\
\hline
\end{tabular}


Chapter 7 

General Discussion 
The importance of lipids to the sustenance of life cannot be over-emphasized. Lipids are a diverse group of biomolecules with different physiological effects. While some forms of lipids carry health and therapeutic benefits, others have been suggested to contribute to cardiometabolic diseases such as hyperlipidaemia, insulin resistance, non-alcoholic fatty liver disease (NAFLD), diabetes, and coronary heart disease. How different types of lipids influence the risk of these diseases has been poorly characterized. Accordingly, there is need for a deeper understanding of the molecular mechanisms regulating lipid metabolism.

The general objective of this thesis was to investigate the regulation of cellular lipid metabolism by trans fatty acids and the lipid-sensitive ANGPTL4 protein. The first aim was to investigate the cellular and molecular mechanisms that underlie the deleterious effects of trans fatty acids on cardiometabolic health using animal and cell culture models. The second aim was to further characterize the role of ANGPTL4 in lipid metabolism, again using animal and cell culture models. Lastly, this thesis examined the effects of muscle-specific inflammation on systemic insulin sensitivity in mice.

The major outcomes of this thesis are that:

- In Chapter 2, we provide an overview of the literature on the molecular effects of trans fatty acids.

- In Chapter 3, we show that a diet enriched in industrial trans fatty acids promotes non-alcoholic fatty liver disease in mice. We also show that the industrial trans fatty acid elaidic acid induces cholesterol synthesis by activating the SREBP signalling pathway.

- In Chapter 4, we demonstrate that ANGPTL4-deficient mice respond more favourably to a diet rich in trans fatty acids than to a diet rich in saturated fatty acids. Furthermore, we show that saturated fatty acids but not trans fatty acids induce inflammation and ER stress in murine macrophages.

- In Chapter 5, using ANGPTL4-hypomorphic mice, we show that a low level of expression of N-terminal ANGPTL4 reduces but does not fully prevent the rise in plasma acute phase proteins and the development of chylous ascites upon high fat feeding. We also show that ANGPTL4 limits lipid uptake and subsequent formation of foam cells in macrophages.

- In Chapter 6, we show that skeletal muscle-specific inflammation via overexpression of the chemokine MCP-1 does not affect systemic insulin sensitivity in mice. 


\section{Industrial trans fatty acids and cardiometabolic disorders}

Epidemiological studies have found a positive association between markers of trans fatty acid intake and the development of NAFLD [1,2]. In chapter 3 of this thesis, we find that a high fat diet enriched in industrial trans fatty acid diet promotes NAFLD in mice. Specifically, we found that feeding C57B1/6 wild-type mice a diet enriched in industrial trans fatty acids promoted liver steatosis and fibrosis along with an increase in liver damage, inflammation and hepatic content of triglycerides and cholesterol, in comparison to diets enriched with saturated or cis-unsaturated fatty acids. In agreement with our study outcome, similar studies have shown that feeding mice diets enriched with industrial trans fatty acids enhances non-alcoholic steatohepatitis (NASH) [3-6]. Hence, both epidemiological studies and studies in mice suggest a stimulatory effect of industrial trans fatty acids on the development of NAFLD.

In addition to NALFD, human studies have also shown a positive association between the intake of industrial trans fatty acids and the development of coronary artery disease [7-10]. This association is likely due to an increase in total and LDL cholesterol and a decrease in HDL cholesterol following intake of foods rich in industrial trans fatty acids [11-13]. In our mice study, we did not see any differences in plasma levels of total cholesterol between the mice fed the various diets. The lack of difference in plasma cholesterol between the various diets may be related to the fact that lipoprotein metabolism is rather different between mice and humans.

In wild-type mice, cholesterol is contained mainly in anti-atherosclerotic HDL, whereas in humans, most of the cholesterol is carried by the pro-atherosclerotic LDL [14]. The difference in lipoprotein metabolism between mice and humans partly stems from the lack of the cholesteryl-ester transfer protein (CETP) in mice $[15,16]$. CETP transfers cholesterol ester from HDL to apoB-containing lipoprotein particles such as VLDL and LDL [16], and has been implicated in the development of atherosclerosis in humans [1719]. Additionally, humans and mice have differences in their lipoproteome [20]. As a result, wild-type mice do not develop spontaneous atherosclerosis, unlike humans. To overcome this limitation, so-called "hum anized" $\mathrm{m}$ ice m odelhave been developed, including ApoE-deficient, LDLr-deficient and APOE*3-Leiden.CETP mice. These mice have similar lipoprotein profiles as humans and develop spontaneous atherosclerosis, making them attractive models to study the molecular mechanisms involved in the development of atherosclerosis, as well as the influence of specific drugs or dietary factors on atherosclerosis [21-23]. Indeed, few studies in LDLr-knockout mice have shown that industrial trans fatty acids increase atherosclerotic plaque formation in the aorta, along with increased plasma levels of total and LDL cholesterol [24], and oxidised 
LDL cholesterol [25]. As indicated above, the absence of an effect of the trans fatty acid diet on plasma cholesterol in our study is likely due to the use of regular C57B1/6 wildtype mice, which contrasts with the reported effects of industrial trans fatty acids on levels of plasma lipoproteins in humans [26].

Interestingly, several studies have reported a positive association between NAFLD and coronary artery disease [27-29]. Currently, however, there is no consensus on a causal relationship between NAFLD and cardiovascular diseases [30,31]. This is largely because NAFLD often co-exists with several other abnormalities such as dyslipidaemia, insulin resistance, and type 2 diabetes, which confounds the association between NAFLD and cardiovascular diseases [32,33]. As indicated above, there is compelling evidence that intake of industrial trans fatty acids promotes NAFLD. Accordingly, it can be speculated that NAFLD may be involved in mediating the positive association between intake of trans fatty acids and coronary artery disease.

\section{Molecular effects between industrial and natural trans fatty acids}

Trans fatty acids can be divided into two main groups; industrial trans fatty acids and natural trans fatty acids. Industrial trans fatty acids are produced artificially by partial hydrogenation of vegetable oils and are contained in snacks and fast food products. Natural trans fatty acids are produced in the gut of ruminant animals by bacterial fermentation of non-degradable fibres and are contained in meat and dairy products [34].

The concentration of trans fatty acids in industrially produced partially hydrogenated vegetable oil can be as high as $60 \%$, with elaidic acid (t18:1n-9) taking up the highest proportion. Meanwhile, the maximum content of trans fatty acids in ruminant fat is approximately $6 \%$. Important natural trans fatty acids include trans vaccenic acid (t18:1n-11), palmitelaidic acid, and the conjugated linoleic acids (CLAs) [35-38].

There is an ongoing debate as to whether industrially produced and naturally produced trans fatty acids exert the same effects on cardiovascular health. A couple of human studies have reported that the harmful effects of trans fatty acids is exclusive to industrial trans fatty acids $[34,35,39]$, whereas natural trans fatty acids are reported to be innocuous or even beneficial to cardiovascular health [40-42]. In contrast, other epidemiological and clinical studies have reported that natural trans fatty acids are equally culpable as industrial trans fatty acids in promoting cardiovascular diseases [43-46].

As part of this thesis, we investigated the molecular effects of industrial and natural trans fatty acids in cell culture experiments to gain mechanistic understanding between these groups of trans fatty acids. In Chapter 4, we showed that compared to untreated controls, elaidic acid did not significantly stimulate inflammatory markers nor ER stress markers 
in RAW264.7 macrophages. In order to determine if the effect of elaidic acid was representative of a broad group of other trans fatty acids, we tested several other natural trans fatty acids. Similar to the elaidic acid effect, trans vaccenic acid, palmitelaidic acid, CLA 9Z,11E and CLA 10E,12Z did not significantly induce expression of inflammatory and ER stress markers in RAW264.7 cells. This result supports the argument that industrial and natural trans fatty acids have similar molecular and cellular effects, at least with respect to their effects on ER stress and inflammation. However, in RAW264.7 macrophages, elaidic acid promoted the expression of genes involved in cholesterol biosynthesis. In Chapter 3, we explored this effect in detail and observed that elaidic acid is cholesterogenic partly because of its ability to stimulate the SREBP/SCAP signalling pathway. Besides increasing the mRNA expression of Srebp2, elaidic acid lowered the levels of intracellular cholesterol and decreased the sensitivity of SCAP to cholesterol. These effects likely underlie the cholesterogenic effects of elaidic acid. Here also, we compared the effects of elaidic acid to natural trans fatty acids. However, the cholesterogenic effects of elaidic acid was not recapitulated by any of the natural trans fatty acids such as trans-vaccenic acid, palmitelaidic acid, CLA 9Z,11E and CLA $10 \mathrm{E}, 12 \mathrm{Z}$. This result supports the claim that industrial and natural trans fatty acids have different molecular and cellular effects.

In conclusion, the outcome of this thesis demonstrates that whereas industrial and natural trans fatty acids share similar molecular effects on ER stress and inflammation, these two types of trans fatty acids have different effects on cholesterogenesis.

\section{Molecular effects between trans-unsaturated and saturated fatty acids}

Saturated and trans-unsaturated fatty acids share common physicochemical properties. The presence of a trans double bond in fatty acids generates a straight chain tertiary structure similar to saturated fatty acids [47,48]. Additionally, trans-unsaturated and saturated fatty acids have relatively high melting temperatures that make them solid at room temperature [49]. Beyond these physicochemical similarities, diets that are high in saturated and trans-unsaturated fatty acids have been reported to negatively alter plasma lipoprotein profiles in humans, characterised by increased levels of total and LDL cholesterol, which is predicted to promote atherosclerosis and coronary artery disease $[11,50]$. Based on these considerations, saturated and trans-unsaturated fatty acids are predicted to have similar effects at the cellular and molecular level.

The studies contained in Chapters 3 and 4 of this thesis highlight the molecular effects of saturated and trans-unsaturated fatty acids and demonstrate that these two types of fatty acids potentially trigger different molecular processes to achieve their 
pathophysiological effects. In Chapter 3, we show in wild-type C57B1/6 mice that a diet rich in industrial trans-unsaturated fatty acids from partially hydrogenated soy oil promotes NAFLD to a greater extent than a diet rich in saturated fatty acids from cocoa butter. At the cellular level, elaidic acid but not palmitic acid strongly induced cholesterogenesis in murine adipocyte and hepatocyte cell lines. In Chapter 4, Angptl4 ${ }^{-1-}$ mice were used to investigate the pro-inflammatory effects of different types of dietary fatty acids. Due to its high sensitivity, Angptl $4^{-/-}$mice are an excellent model to investigate the presumably subtle differences between trans-unsaturated and saturated fatty acids. However, against our expectations, and contrary to the reported harmfulness of industrial trans fatty acids, Angptl $4^{-/}$mice survived 7 weeks of feeding the diet enriched in trans fatty acids, whereas $75 \%$ of the mice ( 6 out of 8 mice) fed the diet enriched in saturated fatty acids became severely ill and died of deleterious proinflammatory complications. Complementary in vitro experiments in RAW264.7 macrophages showed that palmitic acid strongly induced ER stress and inflammation, whereas elaidic acid did not. It is important to reiterate that the effects of all other trans fatty acids on ER stress and inflammation were similar to elaidic acid, while the effect of all other saturated fatty acids on ER stress and inflammation were similar to palmitic acid. In conclusion, although trans fatty acids and saturated fatty acids share a similar structure and are believed to have similar effects, the studies in wild-type and Angptl4-mice and the in vitro experiments revealed pronounced differences between saturated and trans fatty acids.

\section{Molecular effects between trans- and cis-unsaturated fatty acids}

The hydrogenation process that converts PUFAs into trans fatty acids not only leads to differences in physical and structural properties between the cis and trans isomers but also results in profound differences in physiological effects. A number of studies have shown opposing effects between trans and cis-unsaturated fatty acids. In simple terms, cis-unsaturated fatty acids are generally beneficial to health, whereas trans-unsaturated fatty acids are generally harmful. In humans, cis-unsaturated fatty acid diets have been shown to reduce levels of total and LDL-cholesterol, whilst trans-unsaturated fatty acids increase total and LDL-cholesterol [11,50-53]. In a mice study in LDL-receptor knockout mice, a diet containing trans-unsaturated fatty acids increased atherosclerosis development and plaque formation in aorta, whereas a diet containing cis-unsaturated fatty acids showed a protective effect [24].

In Chapter 3 of this thesis, we showed that feeding C57B1/6 mice a diet rich in industrial trans-unsaturated fatty acids leads to more pronounced NAFLD as compared to a diet 
rich in cis-unsaturated fatty acids. Using cell culture experiments, we further demonstrated that elaidic acid is cholesterogenic due to enhanced expression and activity of SREBP2 in murine Hepa1-6 cells. In contrast, oleic acid suppressed expression and activity of SREBP2, leading to strong downregulation of cholesterogenic genes. We recapitulated the differential effects of oleic and elaidic acids in other cells, including murine 3T3-L1 adipocytes and human hepatoma Huh7 cells. Our study corroborates earlier findings from studies in human HepG2 [54] and Huh7 [55] cells, showing prominent induction of cholesterogenesis by elaidic acid, whereas oleic acid had the opposite effect. In Chapter 4 of this thesis, we also showed that the diet enriched with trans-unsaturated fatty acids resulted in formation of giant Touton foam cells in mesenteric lymph nodes of Angptl4 ${ }^{-\digamma}$ mice, whereas the diet enriched in cis-unsaturated fatty acid did not. In deciphering the mechanistic insights, we demonstrated in RAW264.7 macrophages that compared to oleic acid, elaidic acid modestly induced inflammation and ER stress.

In our in vitro studies, oleic acid and elaidic acid served as model cis- and transunsaturated fatty acids, respectively, because they each represent the most abundant fatty acid in their respective groups. Oleic and elaidic acid are 9-octadecenoic acid isomers that differ only by the geometric isomerisation at carbon 9 of their fatty acyl chain [56]. However, the geometric isomerisation confers structural differences between these fatty acids, with oleic acid having a bent and flexible structure, whilst elaidic acid is straighter and rigid [48]. Apparently, this seemingly small structural difference greatly impacts the extent to which these fatty acids are sensed and metabolised, leading to important physiological differences. A number of studies have shown that in comparison to the cis isoforms, incorporation of trans fatty acids into cell membranes alters the membrane composition, leading to a pro-inflammatory response and a dysfunction to endothelial cells [57-59]. Till date, it is still unclear how the cis-trans configuration and the accompanying structural differences affect their metabolism. It is not unimaginable that the cis-trans configuration influences the extent to which these fatty acids can be assembled into triglycerides or hydrolysed into free fatty acids when contained in triglyceride-rich lipoprotein. The structural differences conferred by the cis-trans configuration could impact the efficiency by which enzymes such as lipoprotein lipase access and hydrolyse circulating triglycerides enriched in either cis- or trans-unsaturated fatty acids. Indeed, it has been reported that the cholesterol-ester synthesizing enzyme, acyl coenzyme A:cholesterol-O-acyltransferase (ACAT) discriminates strongly between cis- and trans-unsaturated fatty acids in the formation of cholesterol esters, with a preference for cis-unsaturated fatty acids [60]. 
Mesenteric lymphadenopathy is uncoupled from acute inflammation and chylous ascites in Angptl4 $^{-/}$mice

ANGPTL4 inactivation is clinically beneficial since carrier status of ANGPTL4inactivation mutation is associated with reduced levels of circulating triglycerides and decreased risks of coronary artery disease [61,62]. We have previously shown that Angptl $4^{-/}$mice that were fed a high fat diet enriched with saturated fatty acids develop severe pathological complications that ultimately lead to the death of the animals [63]. The high saturated fat diet generates chylomicron particles which are secreted through the lymphatic system before onward secretion into the blood. The lymphatic system contains mesenteric lymph nodes that are rich in resident macrophages that express relatively high amounts of both $\mathrm{Lpl}$ and Angptl4 [64-66]. Due to deficiency of ANGPTL4, the macrophage LPL activity is unhindered, leading to increased hydrolysis of the triglyceride content of the chylomicron particles. It is believed that the resulting free saturated fatty acids are taken up by the macrophages, resulting in the formation of foam cells that aggregate into a ring-like structure called Touton giant cells. These activated macrophages secrete cytokines that stimulate a pro-inflammatory phenotype through the portal circulation. In response, the liver secretes acute phase markers, including serum amyloid A, haptoglobin and lipocalin. Eventually, chylous ascites and fibrinopurulent peritonitis ensue, that ultimately caused the death of the mice. In our original publication, the formation of Touton giant cells in the mesenteric lymph nodes was presented as the likely initiator of the pro-inflammatory complications in Angptl4 ${ }^{-}$ mice [63].

In Chapter 4, we showed that Angptl4-/mice fed a diet rich in industrial trans fatty acids from partially hydrogenated soy oil develop Touton giant cells in mesenteric lymph nodes to a similar extent as the Angptl $4^{--}$mice fed the saturated fat diet. However, contrary to the saturated fat diet, the trans fat diet did not result in subsequent activation of the acute phase response or chylous ascites, and all the mice survived. Therefore, one major outcome of this thesis is that lymphadenopathy in Angptl4 ${ }^{-/}$mice can be uncoupled from the lethal effects of fibrinopurulent peritonitis and ascites development. This study also affirms that the development of lethal phenotypes in Angptl $4^{-/}$mice is diet-dependent. The differential effect between the trans and saturated fat diets in vivo was partly explained by in vitro experiments. Specifically, palmitic acid and other saturated fatty acids induced a pro-inflammatory phenotype in RAW264.7 macrophages, whereas elaidic acid and other trans-unsaturated fatty acids did not. This suggests that in addition to formation of foam cells, the pro-inflammatory property of the constituent 
fatty acids in the mice diets was important in activating the inflammatory cascade in the Angptl4 ${ }^{-/}$mice.

Even though we learned that mere formation of foam cells in the mesenteric lymph nodes is not enough to activate the pro-inflammatory complications, it is still unclear why the cis-unsaturated fat diet does not lead to formation of Touton giant cells. A possible explanation is that LPL active on the surface of Angptl4-deficient macrophages had different affinities for triglycerides containing different fatty acids. Alternatively, the lipid storage may not only require the provision of substrate but also the presence of a pro-inflammatory stimulus. Saturated fatty acids may carry both properties, while cisunsaturated fatty acids may only serve as substrate. Another possibility is that different fatty acids are differentially taken up by the mesenteric lymph node macrophages.

In conclusion, in light of a clinical study that showed that loss-of-function mutations in ANGPTL4 are seemingly not associated with lymphadenopathies in humans [62], our pre-clinical study suggests that lymphadenopathy could be benign and clinically silent, and therefore downgrades the relevance of this potential side effect of ANGPTL4 inactivation. This observation increases the therapeutic prospect of targeting ANGPTL4 for correcting cardiometabolic abnormalities. Moreover, it can be hypothesized that individuals who will benefit from therapeutic targeting of ANGPTL4 could potentially further reduce the risk of side effects by cutting down the intake of foods rich in saturated fatty acids in favour of foods rich in cis-unsaturated fatty acids.

\section{Clinical relevance of ANGPTL4 based on study in Angptl4-hypomorphic mice}

As highlighted earlier, ANGPTL4 inactivation reduces circulating levels of triglycerides primarily due to enhanced LPL activity, and this is associated with decreased odds of developing coronary artery disease. However, as discussed above, pre-clinical studies in mice revealed damning and ultimately lethal side-effects in whole body Angptl4-/ mice fed a high fat diet enriched with saturated fatty acids. This observation hampers the putative prospects of ANGPTL4 targeting in humans.

In Chapter 5, we make use of Angptl4-hypomorphic mice to further characterize the clinical importance of ANGPTL4. The Angptl4-hypomorphs are characterised by the complete deletion of the C-terminal domain of ANGPTL4, and a partial expression of the N-terminal LPL-inhibiting domain. Compared to wild-type mice, the hypomorphs showed a similar reduction in plasma triglycerides as the whole body Angptl $4^{-/}$mice after a 24-hour fast and after 20 weeks of high saturated fat diet. Additionally, the hypomorphs exhibited greater resistance to a chronic high saturated fat diet by surviving over 36 weeks of feeding, while the Angptl ${ }^{-/}$mice developed pro-inflammatory 
complications and died over the course of the intervention. Only about $12 \%$ of the Angptl4-hypomorphic mice developed chylous ascites, which is markedly lower than the $80 \%$ in the Angptl $^{-/}$mice. Additionally, acute inflammation was significantly lower and delayed in the Angptl4-hypomorphs compared to the Angptl4 $4^{-/}$mice. However, both the hypomorphs and the Angptl4 ${ }^{-/}$mice showed similar levels of Touton giant cell formation in their mesenteric lymph nodes. Since therapeutic targeting of ANGPTL4 will likely reduce instead of completely inactivate ANGPTL4, our hypomorphic model and the outcome of this study are closely applicable to the clinical situation in human.

A number of inferences can be drawn from this study. First, we re-affirm that the formation of Touton giant cells can be uncoupled from fibrinopurulent peritonitis and chylous ascites. We also re-affirm that Touton giant cell formation in mesenteric lymph node macrophages is seemingly benign with little to no clinical impact. Secondly, we demonstrate that clinical targeting of ANGPTL4 with minimal side-effects could potentially be achieved by employing conservative approaches that partially repress instead of completely inactivate ANGPTL4. The modest side-effects in the Angptl4hypomorphs suggests that cardiovascular disease patients who will benefit from ANGPTL4 targeting should have first exhausted other clinical treatments with potentially less side-effects. Our findings in the Angptl4-hypomorphs also indicate that the therapeutic potential of ANGPTL4 targeting can be further fine-tuned to amplify its benefits and downsize its side-effects. This will require a collaborative effort between pre-clinical and clinical studies.

\section{Role of ANGPTL4 in glucose homeostasis and type-2-diabetes}

Recently, several studies have implicated ANGPTL4 in systemic glucose homeostasis and type-2-diabetes. This is not surprising, given that ANGPTL4 is involved in dyslipidaemia, and dyslipidaemia has been positively associated with dysregulated glucose metabolism and incidence of type-2-diabetes [67,68]. Two recent studies have reported that plasma levels of ANGPTL4 are increased in type 2 diabetics $[69,70]$ whilst another study showed that carriers of the E40K loss-of-function mutation in ANGPTL4 have lower odds of developing type 2 diabetes [71]. Using mice models, Gusarova and Janssen have demonstrated in separate studies that Angptl $4^{-/}$mice have lower levels of plasma glucose and improved tolerance compared to wild-type mice [71,72]. In chapter 5 of this thesis, we showed that plasma levels of glucose were significantly lower in Angptl $4^{-/}$mice compared to wild-type mice after 24 hours of fasting or 20 weeks of high fat diet. We also demonstrated by an intra-peritoneal glucose tolerance test that Angptl4${ }^{\wedge}$ mice were more glucose tolerant compared to wild-type mice, while the Angptl4- 
hyopmorphs showed intermediate glucose tolerance between the Angptl4 ${ }^{-/}$and wild-type mice. Although these reports support additional therapeutic benefits of ANGPTL4 inactivation, the mechanism by which ANGPTL4 affects glucose homeostasis is unclear. It is possible that the effect of ANGPTL4 on glucose homeostasis is indirect and secondary to its effect on lipid metabolism. This is likely because the inactivation of ANGPTL4 corrects dyslipidaemia, leading to a more efficient glucose utilisation. However, we cannot rule out the possibility of a direct effect of ANGPTL4 on glucose metabolism. Therefore, further studies will be required to provide mechanistic insights on how ANGPTL4 affects glucose metabolism.

\section{Skeletal muscle inflammation and systemic insulin insensitivity}

Insulin sensitivity is central to diabetes. Accordingly, a detailed understanding of cellular processes that promote insulin insensitivity is vital to enable the identification of molecular targets against diabetes. In light of evidences that inflammatory cytokines interfere with insulin signalling to promote insulin resistance [73,74], the objective of Chapter 6 of this thesis was to investigate the extent to which skeletal muscle inflammation promotes insulin resistance. Using mice that over-express MCP-1 in the skeletal muscle, we demonstrated that skeletal muscle inflammation did not have an effect on systemic insulin sensitivity in both lean and diet induced obese mice. As discussed in Chapter 6, one of the explanations for the differences in tissue-specific inflammatory effect of MCP-1 is glycosylation status of the MCP-1 protein. Additionally, previous studies have shown that adipose-tissue specific inflammation via MCP-1 overexpression reduces whole-body insulin sensitivity and impairs insulinsignalling in skeletal muscle of mice [75,76], suggesting that the global effect of inflammation on systemic insulin sensitivity may be tissue dependent. However, due to the important role of skeletal muscles in glucose disposal in the post-prandial state, the outcome of our study is unexpected and challenges the over-emphasized role of inflammation in systemic glucose homeostasis. Because inflammation is a component of the innate immune system, it is fundamental to many cellular and physiological events. For instance, increased levels of IL-6 and MCP-1 occur in skeletal muscle and plasma during acute exercise [77], and also occurs during chronic low-grade inflammation in obesity [78]. However, existing evidence suggests that these two inflammatory situations can have different and even opposing effects on insulin sensitivity $[79,80]$. Therefore the implication of the "inflammatory hypothesis" as underlying factor of several pathological events must be carefully arrived at based on stronger and compelling evidence. 


\section{Conclusion}

In this thesis, we have extended our understanding of the molecular effects of transunsaturated fatty acids. We demonstrated that compared to diets enriched with cisunsaturated and saturated fatty acids, diets rich in industrial trans fatty acids promote non-alcoholic fatty liver disease characterised by aggravated liver steatosis and fibrosis. At the molecular level, we showed that elaidic acid promotes cholesterogenesis by stimulating the SREBP signalling pathway, which was evident by induced expression of SREBP2 and target genes involved in cholesterol synthesis in a number of cell models including Hepa1-6, Huh7, 3T3-L1 and RAW264.7 cell lines. We also demonstrated that trans fatty acids have molecular effects that are distinct from saturated fatty acids even though these two types of fatty acids share similar physicochemical properties. Additionally, this thesis has demonstrated that in Angptl ${ }^{-/}$mice, enhanced LPL activity increases hydrolysis of triglyceride-rich lipoproteins leading to enhanced uptake of lipids by resident macrophages and a subsequent formation of foam cells in the mesenteric lymph nodes. These foam cells were formed in mice that were fed trans and saturated fatty acid diets, but not cis-unsaturated fatty acid diet. Intriguingly, whereas the formation of foam cells led to inflammation and death in the mice fed the saturated fat diet, there was no further repercussions pertaining to the foam cell formation in the mice fed the trans fat diet. Therefore, we conclude that the formation of foam cells in the mesenteric lymph nodes per se is inadequate to induce inflammation and death, but could be dependent on the properties of fatty acids that generate the foam cells. We have further shown in this thesis that Angptl4-hypomorphic mice characterised by reduced expression of N-terminal exons and complete absence of C-terminal exons of ANGPTL4 survive over 20 weeks of high saturated fat diets. The hypomorphic mice also showed significantly reduced and delayed forms of the lethal phenotypes exhibited in the Angptl4 ${ }^{-/}$mice. The outcome of this study has implications for targeting ANGPTL4 as a measure to reduce the risk of coronary heart disease in humans. 


\section{References}

[1] Mazidi, M., Katsiki, N., Mikhailidis, D.P., Banach, M., 2018. Link between plasma trans-fatty acid and fatty liver is moderated by adiposity. International Journal of Cardiology 272: 316-22.

[2] Mantovani, A., 2018. Plasma trans-fatty acid and risk of nonalcoholic fatty liver disease: New data from National Health and Nutrition Examination Survey (NHANES). International Journal of Cardiology 272: 329-30.

[3] Komatsu, G., Nonomura, T., Sasaki, M., Ishida, Y., Arai, S., Miyazaki, T., 2018. AIM-deficient mouse fed a high-trans fat, high-cholesterol diet: a new animal model for nonalcoholic fatty liver disease. Experimental Animals.

[4] Morinaga, M., Kazuyoshi Kon, ${ }^{*}, \dagger$ Hiroaki Saito, Kum iko Arai, Hirom i Kusam a, A.U., Shunhei Yamashina, K.I. and S.W., 2015. Sodium 4-phenylbutyrate prevents murine dietary steatohepatitis caused by trans-fatty acid plus fructose. Journal of Clinical Biochemistry and Nutrition 56(3): 166-70.

[5] Hu, X., Tanaka, N., Guo, R., Lu, Y., Nakajima, T., Gonzalez, F.J., et al., 2017. $\mathrm{PPAR} \alpha$ protects against trans-fatty-acid-containing diet-induced steatohepatitis. Journal of Nutritional Biochemistry 39: 77-85.

[6] Tetri, L.H., Basaranoglu, M., Brunt, E.M., Yerian, L.M., Neuschwander-Tetri, B.A., 2008. Severe NAFLD with hepatic necroinflammatory changes in mice fed trans fats and a high-fructose corn syrup equivalent. AJP: Gastrointestinal and Liver Physiology 295(5): G987-95.

[7] Mozaffarian, D., Katan, M.B., Ascherio, A., Stampfer, M.J., Willett, W.C., 2006. Trans Fatty Acids and Cardiovascular Disease. New England Journal of Medicine 354(15): 1601-13.

[8] Ganguly, R., Pierce, G.N., 2012. Trans fat involvement in cardiovascular disease. Molecular Nutrition and Food Research 56(7): 1090-6.

[9] Ahmed, S.H., Kharroubi, W., Kaoubaa, N., Zarrouk, A., Batbout, F., Gamra, H., et al., 2018. Correlation of trans fatty acids with the severity of coronary artery disease lesions. Lipids in Health and Disease 17(1): 1-13.

[10] Ascherio, a., Hennekens, C.H., Buring, J.E., Master, C., Stampfer, M.J., Willett, W.C., 1994. Trans-fatty acids intake and risk of myocardial infarction. Circulation 89: 94-101.

[11] Mensink, R.P.\&., Katan, M.B., 1990. Effect of dietary trans fatty acids on highdensity and low-density lipoprotein cholesterol levels in healthy subjects. New England Journal of Medicine 327(2): 82-7.

[12] Katan, M.B., Zock, P.L., Mensink, R.P., 1995. Trans fatty acids and their effects on lipoproteins in humans. Annual Review of Nutrition 15: 473-93.

[13] Judd, J.T., Baer, D.J., Clevidence, B.A., Kris-Etherton, P., Muesing, R.A., Iwane, 
M., 2002. Dietary cis and trans monounsaturated and saturated FA and plasma lipids and lipoproteins in men. Lipids 37(2): 123-31.

[14] Zadelaar, S., Kleemann, R., Verschuren, L., De Vries-Van Der Weij, J., Van Der Hoorn, J., Princen, H.M., et al., 2007. Mouse models for atherosclerosis and pharmaceutical modifiers. Arteriosclerosis, Thrombosis, and Vascular Biology 27(8): 1706-21.

[15] Hogarth, C.A., Roy, A., Ebert, D.L., 2003. Genomic evidence for the absence of a functional cholesteryl ester transfer protein gene in mice and rats. Comparative Biochemistry and Physiology - B Biochemistry and Molecular Biology 135(2): 21929.

[16] Westerterp, M., Van Der Hoogt, C.C., De Haan, W., Offerman, E.H., Dallinga-Thie, G.M., Jukema, J.W., et al., 2006. Cholesteryl ester transfer protein decreases highdensity lipoprotein and severely aggravates atherosclerosis in APOE*3-Leiden mice. Arteriosclerosis, Thrombosis, and Vascular Biology 26(11): 2552-9.

[17] Kuivenhoven, J.A., Jukema, J.W., Zwinderman, A.H., de Knijff, P., McPherson, R., Bruschke, A.V.G., et al., 1998. The Role of a Common Variant of the Cholesteryl Ester Transfer Protein Gene in the Progression of Coronary Atherosclerosis. New England Journal of Medicine 338(2): 86-93.

[18] Klerkx, A.H.E.M., De Grooth, G.J., Zwinderman, A.H., Jukema, J.W., Kuivenhoven, J.A., Kastelein, J.J.P., 2004. Cholesteryl ester transfer protein concentration is associated with progression of atherosclerosis and response to pravastatin in men with coronary artery disease (REGRESS). European Journal of Clinical Investigation 34(1): 21-8.

[19] Boekholdt, S.M., Kuivenhoven, J.A., Wareham, N.J., Peters, R.J.G., Jukema, J.W., Luben, R., et al., 2004. Plasma levels of cholesteryl ester transfer protein and the risk of future coronary artery disease in apparently healthy men and women: The prospective EPIC (European Prospective Investigation into Cancer and nutrition)Norfolk population study. Circulation 110(11): 1418-23.

[20] Gordon, S.M., Li, H., Zhu, X., Shah, A.S., Lu, L.J., Davidson, W.S., 2015. A comparison of the mouse and human lipoproteome: Suitability of the mouse model for studies of human lipoproteins. Journal of Proteome Research 14(6): 2686-95.

[21] van den Hoek, A.M., van der Hoorn, J.W.A., Maas, A.C., van den Hoogen, R.M., van Nieuwkoop, A., Droog, S., et al., 2014. APOE*3Leiden.CETP transgenic mice as model for pharmaceutical treatment of the metabolic syndrome. Diabetes, Obesity and Metabolism 16(6): 537-44.

[22] Getz, G.S., Reardon, C.A., 2016. Do the Apoe-/-and Ldlr-/-mice yield the same insight on atherogenesis? Arteriosclerosis, Thrombosis, and Vascular Biology 36(9): 1734-41.

[23] Meir, K.S., Leitersdorf, E., 2004. Atherosclerosis in the apolipoprotein E-deficient mouse: A decade of progress. Arteriosclerosis, Thrombosis, and Vascular Biology 
24(6): 1006-14.

[24] Machado, R.M., Nakandakare, E.R., Quintao, E.C.R., Cazita, P.M., Koike, M.K., Nunes, V.S., et al., 2012. Omega-6 polyunsaturated fatty acids prevent atherosclerosis development in LDLr-KO mice, in spite of displaying a proinflammatory profile similar to trans fatty acids. Atherosclerosis 224(1): 66-74.

[25] Monguchi, T., Hara, T., Hasokawa, M., Nakajima, H., Mori, K., Toh, R., et al., 2017. Excessive intake of trans fatty acid accelerates atherosclerosis through promoting inflammation and oxidative stress in a mouse model of hyperlipidemia. Journal of Cardiology 70(2): 121-7.

[26] Bendsen, N.T., Chabanova, E., Thomsen, H.S., Larsen, T.M., Newman, J.W., Stender, S., et al., 2011. Effect of trans fatty acid intake on abdominal and liver fat deposition and blood lipids: a randomized trial in overweight postmenopausal women. Nutrition and Diabetes 1(1): e4.

[27] Targher, G., Day, C.P., Bonora, E., 2010. Risk of Cardiovascular Disease in Patients with Nonalcoholic Fatty Liver Disease. New England Journal of Medicine 363(14): 1341-50.

[28] Siddiqui, M.S., Fuchs, M., Idowu, M.O., Luketic, V.A., Boyett, S., Sargeant, C., et al., 2015. Severity of nonalcoholic fatty liver disease and progression to cirrhosis are associated with atherogenic lipoprotein profile. Clinical Gastroenterology and Hepatology.

[29] Ruscica, M., Ferri, N., Macchi, C., Meroni, M., Lanti, C., Ricci, C., et al., 2016. Liver fat accumulation is associated with circulating PCSK9. Annals of Medicine 48(5): 384-91.

[30] Santos, R.D., Valenti, L., Romeo, S., 2019. Does nonalcoholic fatty liver disease cause cardiovascular disease? Current knowledge and gaps. Atherosclerosis.

[31] Bieghs, V., Rensen, P.C.N., Hofker, M.H., Shiri-Sverdlov, R., 2012. NASH and atherosclerosis are two aspects of a shared disease: Central role for macrophages. Atherosclerosis 220(2): 287-93.

[32] Tilg, H., Moschen, A.R., Roden, M., 2017. NAFLD and diabetes mellitus. Nature Reviews Gastroenterology and Hepatology.

[33] Jinjuvadia, R., Antaki, F., Lohia, P., Liangpunsakul, S., 2017. The association between nonalcoholic fatty liver disease and metabolic abnormalities in the United States Population. Journal of Clinical Gastroenterology 51(2): 160-6.

[34] Bendsen, N.T., Christensen, R., Bartels, E.M., Astrup, A., 2011. Consumption of industrial and ruminant trans fatty acids and risk of coronary heart disease: A systematic review and meta-analysis of cohort studies. European Journal of Clinical Nutrition 65(7): 773-83.

[35] Stender, S., Astrup, A., Dyerberg, J., 2008. Ruminant and industrially produced trans fatty acids: Health aspects. Food and Nutrition Research 52: 1-8. 
[36] Wolff, R.L., Precht, D., Molkentin, J., 1998. Trans -18 : 1 Acid Content and Profile in Human Milk Lipids . Critical Survey of Data in Connection with Analytical Methods 75(6): 661-71.

[37] Lock, A.L., Bauman, D.E., 2004. Modifying milk fat composition of dairy cows to enhance fatty acids beneficial to human health. Lipids 39(12): 1197-206.

[38] Ganguly, R., Pierce, G.N., 2015. The toxicity of dietary trans fats. Food and Chemical Toxicology 78: 170-6.

[39] Ascherio, A., Hennekens, C.H., Buring, J.E., Master, C., Stampfer, M.J., Willett, W.C., 1994. Trans-fatty acids intake and risk of myocardial infarction. Circulation 89(1): 94-101.

[40] Jakobsen, M.U., Overvad, K., Dyerberg, J., Heitmann, B.L., 2008. Intake of ruminant trans fatty acids and risk of coronary heart disease. International Journal of Epidemiology 37(December 2007): 173-82.

[41] Bolton-Smith C, Woodward M, Fenton S, B.C., 1996. Does dietary trans fatty acid intake relate to the prevalence of coronary heart disease in Scotland? Eur Heart J. 17(6): 837-45.

[42] Gebauer, S.K., Chardigny, J.M., Jakobsen, M.U., Lamarche, B., Lock, A.L., Proctor, S.P., et al., 2011. Effects of ruminant trans fatty acids on cardiovascular disease and cancer: A comprehensive review of epidemiological, clinical, and mechanistic studies. Advances in Nutrition 2(4): 332-54.

[43] Oomen, C.M., Ocké, M.C., Feskens, E.J.M., Van Erp-Baart, M.A.J., Kok, F.J., Kromhout, D., 2001. Association between trans fatty acid intake and 10-year risk of coronary heart disease in the Zutphen Elderly Study: A prospective population-based study. Lancet 357(9258): 746-51.

[44] Motard-Bélanger, A., Charest, A., Grenier, G., Paquin, P., Chouinard, Y., Lemieux, S., et al., 2008. Study of the effect of trans fatty acids from ruminants on blood lipids and other risk factors for cardiovascular disease. American Journal of Clinical Nutrition 87(3): 593-9.

[45] Brouwer, I.A., Wanders, A.J., Katan, M.B., 2010. Effect of animal and industrial Trans fatty acids on HDL and LDL cholesterol levels in humans - A quantitative review. PLoS ONE 5(3): 1-10.

[46] Gebauer, S.K., Destaillats, F., Dionisi, F., Krauss, R.M., Baer, D.J., 2015. Vaccenic acid and trans fatty acid isomers from partially hydrogenated oil both adversely affect LDL cholesterol: A double-blind, randomized controlled trial. American Journal of Clinical Nutrition 102(6): 1339-46.

[47] Filip, S., Vidrih, R., 2008. Trans Fatty Acids and Human Health 16.

[48] Tvrzicka, E., Kremmyda, L.S., Stankova, B., Zak, A., 2011. Fatty acids as biocompounds: Their role in human metabolism, health and disease - a review. part 1: Classification, dietary sources and biological functions. Biomedical Papers 155(2): 117-30. 
[49] Koletzko, B., Decsi, T., 1997. Metabolic aspects of trans fatty acids. Clinical Nutrition 16(5): 229-37.

[50] Zock, P.L., Katan, M.B., 1992. Hydrogenation alternatives: effects of trans fatty acids and stearic acid versus linoleic acid on serum lipids and lipoproteins in humans. Journal of Lipid Research 33(3): 399-410.

[51] Martin, C.A., Milinsk, M.C., Visentainer, J. V., Matsushita, M., De-Souza, N.E., 2007. Trans fatty acid-forming processes in foods: A review. Anais Da Academia Brasileira de Ciencias 79(2): 343-50.

[52] Mozaffarian, D., Clarke, R., 2009. Quantitative effects on cardiovascular risk factors and coronary heart disease risk of replacing partially hydrogenated vegetable oils with other fats and oils. European Journal of Clinical Nutrition 63(S2): S22-33.

[53] Mozaffarian, D., Aro, A., Willett, W.C., 2009. Health effects of trans-fatty acids: Experimental and observational evidence. European Journal of Clinical Nutrition 63(S2): S5-21.

[54] Vendel Nielsen, L., Krogager, T.P., Young, C., Ferreri, C., Chatgilialoglu, C., Nørregaard Jensen, O., et al., 2013. Effects of Elaidic Acid on Lipid Metabolism in HepG2 Cells, Investigated by an Integrated Approach of Lipidomics, Transcriptomics and Proteomics. PLoS ONE 8(9).

[55] Shao, F., Ford, D.A., 2014. Elaidic acid increases hepatic lipogenesis by mediating sterol regulatory element binding protein-1c activity in HuH-7 cells. Lipids 49(5): 403-13.

[56] Proell, J.M., Mosley, E.E., Powell, G.L., Jenkins, T.C., 2002. Isomerization of stable isotopically labeled elaidic acid to cis and trans monoenes by ruminal microbes. Journal of Lipid Research 43(12): 2072-6.

[57] Niu, S.L., Mitchell, D.C., Litman, B.J., 2005. Trans fatty acid derived phospholipids show increased membrane cholesterol and reduced receptor activation as compared to their cis analogs. Biochemistry 44(11): 4458-65.

[58] Zapolska, D.D., Bryk, D., Olejarz, W., 2015. Trans Fatty Acids and Atherosclerosiseffects on Inflammation and Endothelial Function. Journal of Nutrition \& Food Sciences 05(06).

[59] Harvey, K.A., Arnold, T., Rasool, T., Antalis, C., Miller, S.J., Siddiqui, R.A., 2008. Trans-fatty acids induce pro-inflammatory responses and endothelial cell dysfunction. British Journal of Nutrition 99(4): 723-31.

[60] Sgoutas, D.S., 1970. Effect of geometry and position of ethylenic bond upon acyl coenzyme A-cholesterol-O-acyltransferase. Biochemistry 9(8): 1826-33.

[61] Romeo, S., Pennacchio, L.A., Fu, Y., Boerwinkle, E., Tybjaerg-Hansen, A., Hobbs, H.H., et al., 2007. Population-based resequencing of ANGPTL4 uncovers variations that reduce triglycerides and increase HDL. Nat Genet 39(4): 513-6.

[62] Dewey, F.E., Gusarova, V., O’Dushlaine, C., Gottesm an, O., Trejos, J., Hunt, C., et 
al., 2016. Inactivating Variants in ANGPTL4 and Risk of Coronary Artery Disease. New England Journal of Medicine 374(12): 1123-33.

[63] Lichtenstein, L., Mattijssen, F., de Wit, N.J., Georgiadi, A., Hooiveld, G.J., van der Meer, R., et al., 2010. Angpt14 Protects against Severe Proinflammatory Effects of Saturated Fat by Inhibiting Fatty Acid Uptake into Mesenteric Lymph Node Macrophages. Cell Metabolism 12(6): 580-92.

[64] Dijk, W., Kersten, S., 2014. Regulation of lipoprotein lipase by Angpt14. Trends in Endocrinology and Metabolism 25(3): 146-55.

[65] Georgiadi, A., Wang, Y., Stienstra, R., Tjeerdema, N., Janssen, A., Stalenhoef, A., et al., 2013. Overexpression of angiopoietin-like protein 4 protects against atherosclerosis development. Arteriosclerosis, Thrombosis, and Vascular Biology 33(7): 1529-37.

[66] Makoveichuk, E., Sukonina, V., Kroupa, O., Thulin, P., Ehrenborg, E., Olivecrona, T., et al., 2012. Inactivation of lipoprotein lipase occurs on the surface of THP-1 macrophages where oligomers of angiopoietin-like protein 4 are formed. Biochemical and Biophysical Research Communications 425(2): 138-43.

[67] Parhofer, K.G., 2015. Interaction between glucose and lipid metabolism: More than diabetic dyslipidemia. Diabetes and Metabolism Journal 39(5): 353-62.

[68] Rivellese, A.A., Vaccaro, O., Patti, L., 2004. The pathophysiology of lipid metabolism and diabetes. International Journal of Clinical Practice 58(September): $32-5$.

[69] Abu-Farha, M., Al-Khairi, I., Cherian, P., Chandy, B., Sriraman, D., Alhubail, A., et al., 2016. Increased ANGPTL3, 4 and ANGPTL8/betatrophin expression levels in obesity and T2D. Lipids in Health and Disease 15(1): 1-9.

[70] Nagaike, H., Hayashi, T., Nakanishi, N., Ohara, M., Yamamoto, T., Fukui, T., et al., 2018. Increased Angiopoietin Like Proteins 4 (ANGPTL4) Is Associated with Higher Concentration of LDL-Triglycerides in Type 2 Diabetes. Diabetes 67: 619.

[71] Gusarova, V. et al., 2018. Genetic inactivation of ANGPTL4 improves glucose homeostasis and is associated with reduced risk of diabetes. Nature Communications 9(2252): 1-11.

[72] Janssen, A.W.F., Katiraei, S., Bartosinska, B., Eberhard, D., Dijk, K.W. Van., 2018. Loss of angiopoietin-like 4 ( ANGPTL4) in mice with diet-induced obesity uncouples visceral obesity from glucose intolerance partly via the gut microbiota. Diabetologia 61(6): 1447-1458.

[73] Kahn, S.E., Hull, R.L., Utzschneider, K.M., 2006. Mechanisms linking obesity to insulin resistance and type 2 diabetes. Nature 444(7121): 840-6.

[74] Donath, M.Y., 2014. Targeting inflammation in the treatment of type 2 diabetes: Time to start. Nature Reviews Drug Discovery 13(6): 465-76.

[75] Kamei, N., Tobe, K., Suzuki, R., Ohsugi, M., Watanabe, T., Kubota, N., et al., 2006. 
Overexpression of monocyte chemoattractant protein-1 in adipose tissues causes macrophage recruitment and insulin resistance. Journal of Biological Chemistry 281(36): 26602-14.

[76] Kanda, H., Tateya, S., Tamori, Y., Kotani, K., Hiasa, K.I., Kitazawa, R., et al., 2006. MCP-1 contributes to macrophage infiltration into adipose tissue, insulin resistance, and hepatic steatosis in obesity. Journal of Clinical Investigation 116(6): 1494-505.

[77] Catoire, M., Kersten, S., 2015. The search for exercise factors in humans. FASEB Journal 29(5): 1615-28.

[78] Sindhu, S., Thomas, R., Shihab, P., Sriraman, D., Behbehani, K., Ahmad, R., 2015. Obesity is a positive modulator of IL-6R and IL-6 expression in the subcutaneous adipose tissue: Significance for metabolic inflammation. PLoS ONE 10(7): 1-17.

[79] Lambernd, S., Taube, A., Schober, A., Platzbecker, B., Görgens, S.W., Schlich, R., et al., 2012. Contractile activity of human skeletal muscle cells prevents insulin resistance by inhibiting pro-inflammatory signalling pathways. Diabetologia 55(4): 1128-39.

[80] Bastard, J.-P., Maachi, M., Van Nhieu, J.T., Jardel, C., Bruckert, E., Grimaldi, A., et al., 2002. Adipose tissue IL-6 content correlates with resistance to insulin activation of glucose uptake both in vivo and in vitro. Journal of Clinical Endocrinology and Metabolism 87(5): 2084-9. 

Summary 
Cardiovascular disorders are among the leading causes of death worldwide. Several factors contribute to the risks, onset and progression of cardiovascular diseases, including age, lifestyle, genetics and nutrition. In most instances, a complex interrelationship between two or more of such predisposing risk factors mediate cardiovascular diseases. In this thesis, we have investigated specific aspects of the nutritional and genetic factors, using animal and in vitro models. It is well known that a coordinated regulation of lipid metabolism is important in maintaining cardiometabolic health, since a dysregulation in lipid metabolism is implicated in hyperlipidaemia, insulin resistance, non-alcoholic fatty liver disease (NAFLD), diabetes mellitus and coronary artery disease. Therefore, the aim of this thesis was to investigate the regulation of lipid metabolism with specific focus on trans fatty acids and the lipid-sensitive angiopoietin-like 4 (ANGTPL4) protein.

Epidemiological studies have shown a positive association between the intake of industrial trans fatty acids and cardiovascular diseases. Clinical studies have shown that industrial trans fatty acids perturb plasma lipoprotein metabolism by raising the levels of total and LDL cholesterol, and reducing the levels of HDL cholesterol. This abnormal lipoprotein profile is suggested to mediate the pro-atherogenicity of industrial trans fatty acids. However, only few studies have examined the molecular and cellular mechanisms that underlie the harmful effects of trans fatty acids. Chapter $\mathbf{2}$ of this thesis is a literature review of the studies that examined potential molecular mechanisms of trans fatty acids, mostly in animal and in vitro models. In summary, a number of these studies demonstrate that the harmful effects of trans fatty acids may lie in their negative effect on cellular pathways involved in cellular lipid metabolism, inflammation, autophagy, ER stress and oxidative stress.

In Chapter 3, we showed that C57Bl/6 wild-type mice developed pronounced NAFLD after 7-weeks on a high fat diet enriched with industrial trans-unsaturated fatty acids from hydrogenated soy oil compared to mice fed on cis-unsaturated or saturated fatty acid diets from canola oil and cocoa butter respectively. NAFLD is a liver disease that progresses from a benign state of liver steatosis to non-alcoholic steatohepatitis which is characterised by increased liver inflammation. In the advanced stages of NAFLD, the liver becomes fibrotic leading to liver cirrhosis and hepatocellular carcinoma. In Chapter 3 , we showed that the trans-unsaturated fatty acid diet led to pronounced liver steatosis and fibrosis, increased hepatic inflammation and increased liver damage compared to the mice on the cis-unsaturated and saturated fatty acid diets. Also, in Chapter 3, in vitro studies in murine and human hepatocytes showed that the industrial trans fatty acid, elaidic acid is cholesterogenic. Compared to the cis-unsaturated oleic acid and the 
saturated palmitic acid, elaidic acid increased the mRNA expression and activity of sterol regulatory element binding protein 2 (SREBP2), a transcriptional regulator of cholesterogenic genes. This resulted in an upregulation of important cholesterolsynthesis genes including HMG-CoA reductase. This distinct anabolic property of elaidic acid is a potential contributing mediator to the negative effects of industrial trans fatty acids on cardiometabolic disorders.

The second part of this thesis investigated the role of ANGPTL4 in lipid metabolism. ANGPTL4 is a ubiquitously expressed protein composed of an N-terminal coiled-coiled domain and a C-terminal fibrinogen-like domain. The N-terminal domain of ANGPTL4 is an inhibitor of the extracellular lipase, lipoprotein lipase (LPL). Therefore, ANGPTL4 indirectly elevates plasma triglycerides, which has been shown to be an independent risk factor for cardiovascular diseases. Clinical studies have shown that human carriers of ANGPTL4 variants have reduced odds of developing coronary artery disease. Therefore, ANGPTL4 inactivation is a therapeutic potential for correcting dyslipidaemia and associated cardiovascular diseases. However, we and others have shown that in response to high saturated fatty acid diet, Angptl4 ${ }^{-/}$mice develop lethal phenotypes characterised by formation of Touton giant cells in mesenteric lymph nodes, chylous ascites and peritonitis, that ultimately led to death. These deleterious phenotypes are potential sideeffects of ANGPTL4 inactivation that hamper the prospect of ANGPTL4 targeting. Due to the reported similarities between trans-unsaturated and saturated fatty acids, we hypothesized in Chapter 4 that Angptl $4^{-/}$mice will respond similarly to high fat diets enriched in either trans-unsaturated or saturated fatty acids. Contrary to our hypothesis, the Angptl $4^{-/}$mice on the saturated fat diet got severely ill and died, while the mice on the trans fat diet survived the intervention with no physical signs of ill-health. The saturated fat diet led to the formation of Touton giant cells, chylous ascites and acute inflammation as was previously observed, whereas the mice on the trans fat diet showed no signs of acute inflammation or peritonitis. However, the trans fat diet resulted in formation of Touton giant cells in the mesenteric lymph nodes to similar levels as the saturated fat diet. We had previously suggested that the formation of Touton giant cells is coupled to acute inflammation and chylous ascites. However, this observation in the trans fat fed Angptl4 ${ }^{-/}$mice led to an important finding and two conclusions: (1) the formation of Touton giant cells can be uncoupled from acute inflammation and chylous ascites and (2) mesenteric lymphadenopathy can be clinically silent. Complementary in vitro experiments in RAW264.7 macrophage-like cells showed induction in gene markers of inflammation and ER stress by palmitic acid but not elaidic acid, further revealing molecular differences between saturated and trans-unsaturated fatty acids. 
In Chapter 5, we further investigated the clinical potential of targeting ANGPTL4 by using an Angptl4-hypomorphic mice model that had a complete deletion of the Cterminal domain of ANGPTL4, and a partial expression of the N-terminal LPL-inhibiting domain. Compared to wild-type mice, the hypomorphs showed only about $30-50 \%$ mRNA expression of exons 1 to 3 which contains the N-terminal domain of ANGPTL4, leading to a truncated ANGPTL4 protein. We compared physiological response of the hypomorphs to Angptl4 $4^{--}$and wild-type mice after 20-weeks of high fat diet and after 24-hours of fasting. Both Angptl4-hypomorphic and Angptl4 ${ }^{-/}$mice showed similar levels of reduced plasma triglycerides in comparison to the wild-types after 24-hour fast or 20-weeks on high fat diet. While the high fat diet intervention expectedly resulted in pronounced formation of chylous ascites in about $85 \%$ of the Angptl4 $^{-/}$mice, chylous ascites formed in appreciably lower volumes, and in only about $12 \%$ of the Angptl4hypomorphic mice. Additionally, there was delayed onset and significantly reduced acute inflammation in the Angptl4-hypomorphic mice compared to the Angptl4 ${ }^{-/}$mice. However, both the hypomorphs and the Angptl4 ${ }^{-/}$mice showed similar formation of lipid-laden Touton giant cells in mesenteric lymph nodes. These findings are of clinical importance, since therapeutic targeting will potentially reduce but not completely inactivate ANGPTL4. Also in Chapter 4, we showed that increased LPL activity due to ANGPTL4-deficiency in primary macrophages, promoted foam cell formation and induced the expression of gene markers of ER stress and inflammation after incubation of the ANGPTL4-deficient macrophages with triglyceride-rich lipid particles.

In Chapter 6, we explored the effect of muscle-specific inflammation on systemic insulin sensitivity in mice. Insulin insensitivity underlies diabetes mellitus. Previous studies showed that adipose tissue-specific inflammation promote systemic insulin resistance and generated abnormal glucose homeostasis. Due to the important role of skeletal muscle in glucose disposal in the post-prandial state, we hypothesized that skeletal muscle-inflammation will promote insulin resistance. Using a transgenic mice model that over-expressed the chemokine MCP-1 under the creatinine kinase promoter, we showed that muscle specific over-expression of Mcp-1 strongly induced local inflammation in the skeletal muscles in both lean and diet-induced obese mice. However, both lean and diet-induced obese mice showed no differences in systemic glucose and insulin sensitivity measured by an intraperitoneal glucose and insulin tolerance tests respectively. This led to the conclusion that skeletal-muscle inflammation via $M c p-1$ overexpression has no effect on systemic insulin sensitivity in mice.

In conclusion, the studies performed in this thesis have increased our understanding of the molecular effects of trans fatty acids and the regulatory effects of ANGPTL4 in lipid 
metabolism. We have demonstrated that an industrial trans fatty acid diet promotes NAFLD in mice. We have also shown that trans fatty acids have distinct physiological and molecular effects from saturated fatty acids. Based on in vitro studies, we have demonstrated that saturated fatty acids promote ER stress and inflammation whereas industrial trans fatty acids have a cholesterogenic property. Response by ANGPTL4deficient mice to trans fat diet has revealed that chylous ascites and acute inflammation can be uncoupled from mesenteric lymphadenopathy. And by characterizing the physiological response of an Angptl4-hypomorphic mice to high fat diet and fasting, we have demonstrated that potential side-effects of ANGPTL4 targeting could be reduced significantly but not completely by clinical strategies that will partially repress instead of strategies that will completely inactivate ANGPTL4. 

"If you were successful, somebody along the line gave you some help, there was a great teacher somewhere in your life"- Barack Obama, 2012.

For almost 7 years, I have been associated with Wageningen University. Here, I received both my Masters' degree in Molecular Life Science and now, a PhD degree in Molecular Nutrition. Without a Bachelor and a Master, a PhD would be impossible. Therefore, I will start by expressing gratitude to my mentor and supervisor during my undergraduate studies, Dr Jonathan P. Adjimani of the Department of Biochemistry, Cell and Molecular Biology, University of Ghana for seeing a good in me. I appreciate the support of the Ghana Education Trust Fund (GETFund) for sponsoring my Masters' studies. I also thank the study advisor of my Masters' program, Dr Joan Wellink of the Molecular Biology group, Wageningen University for his timely and steering counsels. I am also grateful to my thesis supervisors, Dr Kishan Naipal and Dr Dik van Gent of the Molecular Genetics Department, Erasmus Medical Centre-Rotterdam, for setting me up for life in the lab. Also, thank you to Dr Anneke Blackburn of the John Curtin School of Medical Research, Australian National University for her supervision and help during my internship in Canberra, Australia.

Without a doubt, the person who has made the biggest impact on my academic life and on my career is my PhD supervisor and promotor, Professor Sander Kersten. For starters, nutrition related research was not really my thing, and my background and prior research interests said it all. However, during a brief volunteering stint in Sander's lab, I developed interest in this area of research. I was pleasantly surprised when Sander offered me a $\mathrm{PhD}$ opportunity in his group. This was a big gamble and risk, much on Sander's part than on my part. My drive to succeed was borne out of desire to reciprocate the faith Sander vested in me. For the last 4 and half years, it has been an honour to have Sander as my supervisor. Sander's high expectations and uncompromising desire for quality helped me up my game, even if slightly by his standards. From having to choose beautiful colours for my figures, to apt alignment of figures, to making "crisp" microscope pictures, to working till late to learn to use CorelDraw, to my futile efforts of reading my written articles over and over again with hope that it comes back with less "red marks". This has been an invaluable experience and I am privileged to have acquired this from the best supervisor I could ever have. Thank you Sander!

Montse, it has been a real pleasure to have you as a colleague. We started our $\mathrm{PhD}$ about the same time and took few courses together. I remember our Animal Handling course in Utrecht and how we had to shelf our lab work and journey to Utrecht early in the mornings, every day for 2 weeks. Many thanks for radiating so much calm and encouragement, it was always nice to talk to you about anything. Your happy attitude 
and beautiful friendliness are infectious. I will miss you. I will be very lucky to find a future colleague who possesses even half of your vibes. Or maybe the solution is that you finish soon, find a job, so I can apply for a position at your work place :-)

Philip, our lunch times together often turned into detailed discussions of each other's newest lab data. It was possible that we could swap projects seamlessly. It was also nice that we worked together on a shared project. Aside work, we had good times playing football on Friday evenings, discussing World Cup games, watching Champions league football and playing FIFA. Our many discussions will continue for sure even after our time in Wageningen ends. For now, good luck with the rest of the PhD. And thanks for agreeing to be my Paranymph!

Miranda, thank you for agreeing to be my Paranymph and for helping me with final preparations towards my $\mathrm{PhD}$ defence. I am glad that I drew inspiration from you to learn to use Adobe Illustrator. But above all, thank you for being a nice office mate. I hope that you achieve lots of success in the remainder of your PhD work.

Rogier, we shared the same office, first at de Valk and later in Helix. We had several interesting discussions on diverse topics including providing each other with second opinions on data from our studies. The more interesting discussions were on our future plans, marriage, raising kids, holiday plans, buying a new bike, what to do with Optare, about everything imaginable :-). Our office turns into female dominance since our contracts end about the same time. Good luck buddy with future plans.

Pol, it was nice that we could to do some lab experiments together and work together in the Newtrition newsletter team. Even nicer memories of our Wembley and Emirates football-watching experience during the UK PhD tour. I will miss our Friday evening football and the Champions League nights, the pizza and our FIFA games! I wish you success with the rest of your $\mathrm{PhD}$.

Danny, Lisa, Merel and Xanthe, it's been a pleasant experience to have you guys as colleagues. It was always nice that we could discuss details of our lab projects and help each other out when the need be. And thank you for the many interesting conversations. I wish you loads of luck with the rest of your $\mathrm{PhD}$ and lots of successes with future plans.

Iris and Lee, thank you for the nice little chats in the office that helped blow off some steam during busy working days. I wish you lots of luck in your work.

Mara, Anouk, Charlotte, Pim and Tessa, I wish you lots of success in finishing your $\mathrm{PhD}$ and with your future endeavours. 
Sheril, Wieneke, Aafke and Lily, you were good examples in the time we shared as colleagues. Thanks for the times when you patiently helped me out in the lab and offered nice tips for my work. I wish you successes in your current job positions.

Ja and Inge, it was nice to share office space with you both. I wish you successes and good luck at your current jobs.

Guido, it was always nice to discuss my work with you, to receive feedback, suggestions and interesting perspectives for improvement. Thanks for the help in analysing some of my microarray data and for the many interesting side-chats.

Mark, I am grateful to you for reading portions of my thesis and providing me with useful feedback. Even though my request for help was at such a short notice, you gladly assisted me which contributed in making me meet the submission deadline. Thank you! Rinke, any knowledge I have on Immunometabolism is thanks to your very nice and clear presentations. Thanks also for your useful suggestions and comments on my work.

Wilma, it was a nice experience working with you on the student practical course. I learnt a lot, thank you!

Lydia, Jocelijn and Klaske, thank you for your nice questions, suggestions and feedbacks on my work during the Thursday morning group meetings. I wish you successes with your projects.

Mieke, thanks a lot for the help in the cell culture lab at the start of my PhD and the very interesting conversations about lab work and marriage :-). And oh, bedankt voor de Nederlandse les.

Shohreh, I appreciate your assistance in the lab, especially on my histology experiments. And thanks also for all the cookies, fruits and the many little chats.

Karin, thanks a lot for helping me out with genotyping and picking up samples for me from the Biobank irrespective of the many times I came asking.

Jenny, Mechteld, Marlies and Carolien, thank you for the lab tips, your help in placing orders, organising the lab and the assistance that helped me get my experiments running smoothly.

To my previous students: Marina, Edwyn, Roger, Aditya, Dominique and Imke, it was nice working with you and an honour to supervise your thesis/internships. Thank you for the assisting me in the lab. I wish you great successes in your next adventures.

And to my supportive dad, Samuel Benson Oteng and mum, Grace Akua Konadu, as well as my siblings and relatives far and near who have wished me well on this journey, 
I say a big thank you! Special appreciation to Esther, my darling wife and my in-laws for their unceasing support and encouragement.

As a devout Christian, I am and will always remain grateful to God Almighty for direction, inspiration and sustenance through the good and difficult times. To my Christian family in Wageningen, at the International Christian Fellowship (ICF), Amazing Grace Parish (AGP) and past and current members of the Asserpark Connect Group of ICF, God bless you for being a wonderful company of spiritual and social support.

Thanks to everyone who has cared for and invested in me, you will not be disappointed! 

About the author $x^{2}$ 


\section{Curriculum Vitae}

Antwi-Boasiako Oteng was born on March 20, 1988 in Kumasi, Ghana. He started basic school at Joy Standard School and later to Living Waters Basic School both in Kumasi. In 2003, Antwi started his secondary school education in Opoku Ware School where he studied General Science. In his second year, he won awards as the Best Physics student and overall Best Science Student during the school's Speech and Prize Day. In 2007, Antwi gained admission to study Biological Science at the University of Ghana in Accra. He specialised in Biochemistry and became interested in cellular and molecular level research that aims to identify novel pharmacological targets against chronic diseases such as cancer and cardiovascular disorders. Motivated by this, Antwi applied and gained admission in 2012 to study a Masters' program in Molecular Life Science at Wageningen University. He specialised in Biomedical Research with a Master thesis research on cancer genetics at Erasmus Medical Centre, Rotterdam-Netherlands. Antwi then took an internship that researched into the metabolism of cancer cells, at the John Curtin School of Medical Research in Canberra, Australia. After completion of his Masters' degree and in April 2015, Antwi started as a PhD candidate in the Nutrition, Metabolism and Genomics group at Wageningen University. Under the supervision of Prof. Sander Kersten, Antwi's work focussed on the cellular metabolism of trans fatty acids as well as new insights into the role of angiopoietin-like 4 (ANGPTL4) in lipid metabolism. The findings of his research are contained in this thesis. After completion of his $\mathrm{PhD}$ program, Antwi will start as a post-doctoral research scientist in the lab of Dr Rebecca Haeusler at the Department of Pathology \& Cell Biology, Columbia University Medical Centre, New York, USA. 


\section{List of Publications}

\section{This thesis}

Oteng AB*, Ruppert PMM*, Boutens L, Dijk W, Stienstra R, Kersten S. Angpt14 hypomorphic mice expressing truncated N-terminal ANGPTL4 exhibit lymphadenopathy and mild chylous ascites upon high fat feeding. Submitted

Oteng AB, Kersten, S. Molecular effects of trans fatty acids. In preparation

Oteng AB, Loregger A, van Weeghel M, Zelcer N, Kersten S. Industrial trans fatty acids promote non-alcoholic fatty liver disease and stimulate SREBP2-mediated cholesterogenesis. Submitted.

Oteng AB, Bhattacharya A, Brodesser S, Qi L, Tan NS, Kersten S. (2017). Feeding Angptl4 ${ }^{\digamma}$ mice trans fat promotes foam cell formation in mesenteric lymph nodes without leading to ascites. J Lipid Res. 58(6):1100-1113.

Evers-van Gogh IJA*, Oteng AB*, Alex S, Hamers N, Catoire M, Stienstra R, Kalkhoven E, Kersten S (2016). Muscle-specific inflammation induced by MCP-1 overexpression does not affect whole-body insulin sensitivity in mice. Diabetologia. 59: $624-633$.

\section{Others}

Grootswagers P, Smeets ETHC, Oteng AB, de Groot LCPGM. The effects of a novel oral nutritional supplement as compared to standard care on body composition, physical function and skeletal muscle mRNA expression in Dutch older adults with (or at risk of) undernutrition. In preparation

* Equal contribution 


\section{Overview of completed training activities}

\section{Discipline specific activities}

Courses \& Meetings

- NutriScience (Wageningen, The Netherlands, 2015)

- CVON-Energise (The Hague, The Netherlands, 2015)

- Energy Metabolism \& Body Composition (Wageningen, The Netherlands, 2016) - Oral presentation

- CVON-Energise (Leiden, The Netherlands, 2016)

- Atherosclerosis \& Thrombosis, DHF (Papendal, The Netherlands 2016)

- CVON-Energise (Wageningen, The Netherlands, 2016)

- CVON-Energise, Young Talent Forum (Utrecht, The Netherlands, 2017)

- Nutrition Science Days (Heeze, The Netherlands, 2017) - Oral presentation

- CVON-Energise (Wageningen, The Netherlands, 2018) - Oral presentation

- CVON-Energise (Leiden, The Netherlands, 2018) - Poster presentation

- CVON-Energise, Young Talent Forum (Wageningen, The Netherlands, 2018)

\section{Conferences}

- Diabetes Research Meeting, NVDO (Oosterbeek, The Netherlands, 2015) - Oral presentation

- Diabetes Research Meeting, NVDO (Oosterbeek, The Netherlands, 2016)

- European Lipoprotein Club, EAS (Tutzing, Germany, 2017) - Oral presentation

- XX Lipid Meeting (Leipzig, Germany, 2017) - Oral presentation

- Kern Lipid Conference (Vail, Colorado, USA, 2018) - Poster presentation

\section{General courses}

- $\quad$ PhD Workshop Carousel, WGS (Wageningen, The Netherlands, 2015)

- VLAG PhD week, VLAG (Baarlo, The Netherlands, 2015)

- Reviewing a Scientific Paper, WGS (Wageningen, The Netherlands, 2015)

- Laboratory Animal Science course (Utrecht, The Netherlands, 2016)

- Applied Statistics, VLAG (Wageningen, The Netherlands, 2016)

- Scientific Writing (Wageningen, The Netherlands, 2015) 


\section{Optional courses/activities}

- Preparation of Research proposal (Wageningen, The Netherlands, 2015)

- NMG/Pharma Lab meetings (Wageningen, The Netherlands, 2015-2019)

- PhD Study Tour to United Kingdom (including organization, 2017) 
The research contained in this thesis was financially supported by the Graduate School VLAG (Advanced studies in Food Technology, Agrobiotechnology, Nutrition and Health Sciences).

I gratefully acknowledge the financial support by Wageningen University and VLAG for the printing and publishing of this thesis

Cover: Antwi-Boasiako Oteng \& Dennis Hendriks (Proefschriftmaken)

Layout: Antwi-Boasiako Oteng

Printed by: Digiforce || proefschriftmaken.nl 



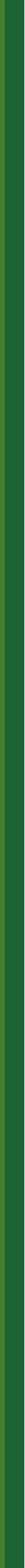

